\title{
Skeletal muscle health in aging
}

Citation for published version (APA):

Grevendonk, L. (2021). Skeletal muscle health in aging: a focus on mitochondria, metabolism and physical performance. [Doctoral Thesis, Maastricht University]. Maastricht University. https://doi.org/10.26481/dis.20211006lg

Document status and date:

Published: 01/01/2021

DOI:

10.26481/dis.20211006lg

Document Version:

Publisher's PDF, also known as Version of record

\section{Please check the document version of this publication:}

- A submitted manuscript is the version of the article upon submission and before peer-review. There can be important differences between the submitted version and the official published version of record.

People interested in the research are advised to contact the author for the final version of the publication, or visit the DOI to the publisher's website.

- The final author version and the galley proof are versions of the publication after peer review.

- The final published version features the final layout of the paper including the volume, issue and page numbers.

Link to publication

\footnotetext{
General rights rights.

- You may freely distribute the URL identifying the publication in the public portal. please follow below link for the End User Agreement:

www.umlib.nl/taverne-license

Take down policy

If you believe that this document breaches copyright please contact us at:

repository@maastrichtuniversity.nl

providing details and we will investigate your claim.
}

Copyright and moral rights for the publications made accessible in the public portal are retained by the authors and/or other copyright owners and it is a condition of accessing publications that users recognise and abide by the legal requirements associated with these

- Users may download and print one copy of any publication from the public portal for the purpose of private study or research.

- You may not further distribute the material or use it for any profit-making activity or commercial gain

If the publication is distributed under the terms of Article $25 \mathrm{fa}$ of the Dutch Copyright Act, indicated by the "Taverne" license above, 
Skeletal muscle health in aging: a focus on mitochondria, metabolism and physical performance. Lotte Grevendonk.

ISBN: 978-94-6423-369-8

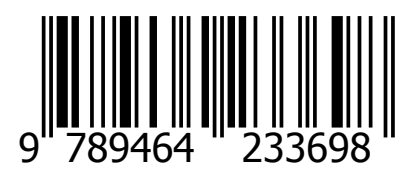

Cover and layout design: An-Sofie Hendrix, ansofie.hendrix@drukkerijhendrix.be Printing: Drukkerij Hendrix

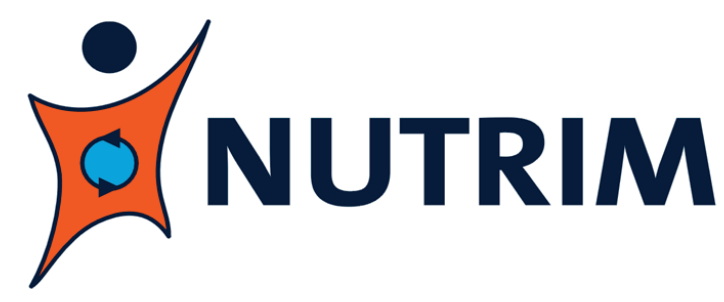

This dissertation and all the studies included were performed within NUTRIM School of Nutrition and Translational Research in Metabolism.

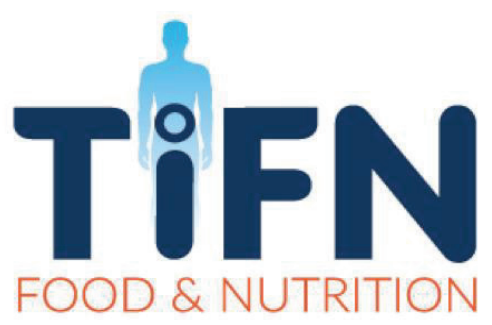

Chapters 3, 4, 5 and 6 presented in this thesis were performed within the framework of TiFN.

Copyright $\odot$ Lotte Grevendonk, 2021, Maastricht, the Netherlands. 


\section{Skeletal muscle health in aging}

A focus on mitochondria, metabolism and physical performance

\section{Dissertation}

to obtain the degree of Doctor at the Maastricht University, on the authority of the Rector Magnificus, Prof. dr. Rianne M. Letschert in accordance with the decision of the Board of Deans, to be defended in public on Wednesday, October 6, 2021, at 13:00 hours.

By

\section{Lotte Grevendonk}

Born in Lommel, Belgium, on May 27, 1991 


\section{Promotors:}

Prof. dr. P. Schrauwen

Prof. dr. R. Houtkooper (Amsterdam UMC)

\section{Copromotor:}

Dr. J. Hoeks

\section{Assessment Committee:}

Prof. dr. S. Kremers (Chairman)

Prof. dr. ir. A. Schols

Prof. dr. ir. J. Keijer (Wageningen University \& Research)

Dr. P. Coen (Translational Research Institute for Metabolism and Diabetes, Orlando)

Dr. T. Adam 


\section{TABLE OF CONTENTS}

Chapter 1 General Introduction and Outline

Chapter 2 Skeletal muscle mitochondrial network dynamics in human metabolic disorders and aging

Chapter 3 Impact of aging and exercise on skeletal muscle 45 mitochondrial capacity, energy metabolism, and physical function

Chapter 4 Healthy aging and muscle function are positively associated to $\mathrm{NAD}^{+}$abundance in humans

Chapter $5 \quad \mathrm{NAD}^{+}$-precursor supplementation with L-tryptophan, nicotinic acid, and nicotinamide does not affect mitochondrial function or skeletal muscle function in physically compromised older adults

Chapter 6 Type II muscle fibre properties are not associated with balance recovery following large perturbations during walking in young and older adults

Chapter 7 General discussion

Appendices Samenvatting

Summary

Sommario

Impact

Dankwoord

About the author

List of publications 


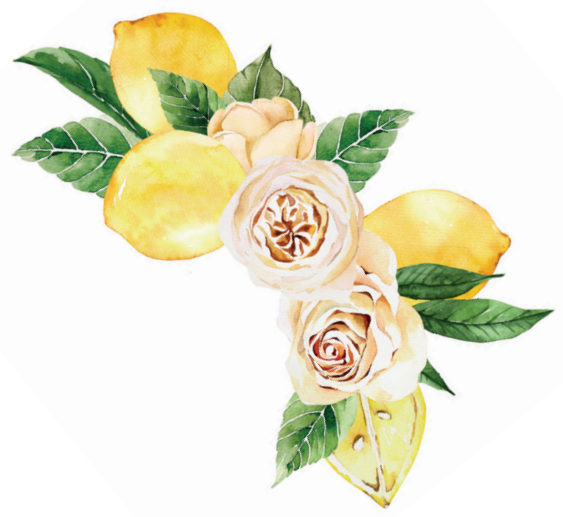

\section{CHAPTER 1}

General introduction and outline

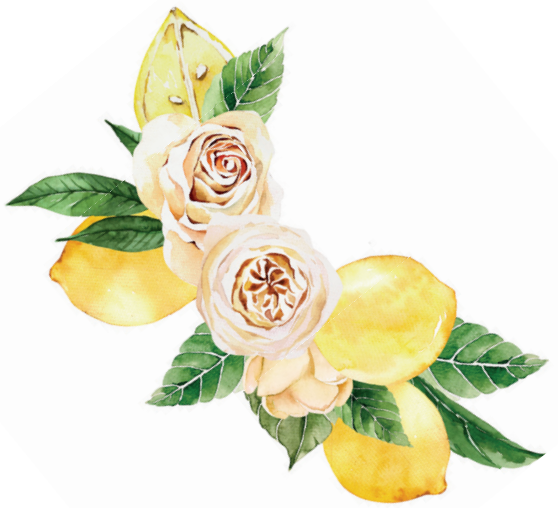




\section{WORLD'S AGING POPULATION}

People live longer than ever before and as a result, the world is expected to experience a significant global population aging over the coming years $(1,2)$. Essentially every country in the world is encountering growth in the proportion of older persons in its population. In 2019, 703 million people were aged over 65 years in the global population, and this number is predicted to double to 1.5 billion in 2050 (3). The share of the worldwide population aged over 65 years increased from 6 percent in 1990 to 9 percent in 2019 and is expected to rise further to 16 percent in 2050, meaning that by that time, one in six people worldwide will be aged 65 years or older (3). This increase in human longevity reflects the remarkable advances achieved in healthcare, nutrition, and biomedicine over the past century. Nevertheless, the increase in lifespan is not paralleled by similar gains in health span since chronological age is a significant risk factor for chronic diseases such as cancers, diabetes, and cardiovascular/respiratory diseases (4-8). As a result, the globally aging population implicates significant challenges for health, long-term care, and welfare systems. Aging is also associated with a loss of independence, and new solutions are urgently needed to sustain self-management at higher ages $(1-3,9,10)$. Understanding and targeting the aging process itself, rather than treating the symptoms of age-related pathologies, might be the most effective strategy to prevent or counteract aging-related health issues. One of the main processes with aging is the progressive loss of muscle mass and physical function, known as sarcopenia (11). Sarcopenia is often associated with impaired mobility, greater tendency to fall, loss of independence, functional limitations, metabolic impairments, and increased risk of chronic diseases and causal mortality (12-18).

Unhealthy aging also relates to frailty, which is defined as a loss of physical function and an increased susceptibility to adverse health outcomes $(19,20)$. Although the biology of frailty is not yet well understood, skeletal muscle health is critical to prevent physical frailty and to maintain health at a higher age $(19,21,22)$. Frailty is linked to the loss of muscle mass and muscle strength, and longitudinal studies show that in adults aged over 75 years, muscle mass is lost at a rate of $0.64-0.70 \%$ per year in women and $0.80-0.98 \%$ per in men, while in women men aged over 18 years this on average is $0.37 \%$ per year and $0.47 \%$ per year, respectively. Even more prominent, muscle strength is lost at a rate of $2.5-3 \%$ and $3-4 \%$ per year in adult 
women and men, respectively. The loss of strength is $2-5$ times faster than the loss of muscle mass and is a more consistent risk for disability (7). In addition, skeletal muscle is also a major site of metabolic activity that regulates protein, lipids, and carbohydrates homeostasis. For instance, skeletal muscle is responsible for the large majority of post-prandial glucose disposal $(23,24)$, and failure in these metabolic processes can lead to perturbations in glucose homeostasis, including hyperglycemia and insulin resistance. Insulin sensitivity is a significant determinant for the risk of many chronic metabolic diseases.

Moreover, aging is one of the major risk factors for chronic metabolic disorders such as obesity, type 2 diabetes, and cardiovascular diseases (25). A functional mitochondrial network in skeletal muscle tissue is essential to sustain the metabolic demands and maintain insulin sensitivity by regulating fuel utilization and energy expenditure. Interestingly, several (pre-) clinical studies suggest that improvements in various aspects of mitochondrial function are linked to the preservation of muscle mass, metabolism, and muscle function (26-29), putting mitochondria at the center of muscle health and physical function.

\section{ROLE OF MITOCHONDRIA AND PHYSICAL ACTIVITY IN AGING}

Mitochondria are highly dynamic organelles that play an essential role in adjusting cellular energy production to metabolic demands (30). In parallel with the progressive loss of muscle function, a decrease in skeletal muscle mitochondrial respiratory activity and dysregulation of mitochondrial network dynamics have been shown with advancing age (31-36). Experimental evidence has liked aberrant mitochondrial quality control to elevated reactive oxygen species emission and activation of apoptotic pathways thought to contribute to sarcopenia's pathophysiology (37). Furthermore, mitochondrial dysfunction has recently been indicated as an underlying factor for low physical function (38), slower walking speed (39-41), fatigability (42), and loss of exercise efficiency with older age (43). However, the age-associated decline in mitochondrial function in humans might not be entirely attributable to aging per se. It may also be explained, in part, by an agerelated decline in physical activity $(44,45)$. Thus, evidence indicates a negative relationship between age and physical activity (46), and only $23.5 \%$ of the aging population succeeds in meeting the WHO recommended physical activity levels of at least 150 minutes of moderate-intensity aerobic physical activity per week (47). 
Irrespective of the role of mitochondria in influencing physical function and muscle health with aging, physical exercise is a powerful tool to protect against aginginduced deterioration of muscle health (48) and the onset of numerous age-related chronic diseases $(49,50)$.

\section{THESIS OUTLINE}

The present thesis aims to investigate the role of skeletal muscle mitochondria and physical activity in the age-related loss of physical function and metabolic health. Such information is instrumental in revealing promising therapeutic strategies to promote healthy aging. In recent years it has become clear that mitochondria do not operate as individual organelles but as dynamic networks. As such, mitochondrial function is not only defined by intrinsic bioenergetic capacity but also includes the ability of mitochondria to move and physically interact with each other in dynamic reticular networks. This plasticity in mitochondrial morphology enables the mitochondria to rapidly adapt to dynamic changes in the cell's metabolic demands and is fundamental to a wide variety of cellular functions (51). Recent evidence suggests that aberrant changes in mitochondrial network dynamics occur with aging and this evidence is examined in the review of chapter 2 of this dissertation.

Considering these age-related changes in mitochondrial network dynamics, it has been suggested that the decline in mitochondrial capacity might explain the accelerated loss in muscle health with age. However, it remains unclear to which extent age-related physical inactivity and chronological age per se contribute to the decline in mitochondrial function and physical function during aging. To investigate this relationship between mitochondrial and physical function, in chapter 3, we extensively analyzed mitochondrial capacity and muscle function in young and older adults with similar habitual physical activity levels. Moreover, we studied older adults with a range of habitual physical activity levels and muscle function, i.e. trained older adults, older adults with normal physical activity levels, and physically impaired older adults.

A large number of preclinical studies have linked alterations within mitochondrial metabolism to the aging process. As mitochondria play a crucial role in energy metabolism homeostasis, mitochondrial dysfunction might explain age-related metabolic complications such as cardiovascular diseases, obesity, and type 2 diabetes $(52,53)$. Furthermore, chronological age is associated with distinct plasma 
metabolic profiles $(54,55)$, providing interesting leads to identify pathways that may affect lifespan. However, since the rate at which physiological function decline with age is highly variable among individuals (56), these metabolic profiles do not necessarily reflect the age-related decline in functional health outcomes and hence may not mirror the biological age. To further identify the metabolic changes in skeletal muscle related to chronological age, in chapter 4 we studied the muscle metabolome in young vs. older adults. Moreover, the impact of biological age on the skeletal muscle metabolome was examined by investigating different groups of older individuals representing healthy, normal and impaired aging. This examination revealed a strong decline of the nicotinamide dinucleotide $\left(\mathrm{NAD}^{+}\right)$with age, which was exacerbated with impaired aging and nearly entirely prevented in healthy aging, indicating $\mathrm{NAD}^{+}$as a promising therapeutic target to promote healthy aging. To further explore the therapeutic potential of $\mathrm{NAD}^{+}$targeting, chapter $\mathbf{5}$ of this dissertation investigates the efficacy of a nutritional NAD ${ }^{+}$pre-cursor supplementation on mitochondrial function in older adults with impaired physical function.

One of the most critical consequences of aging is the loss of gait and balance control, inducing an increased risk of falls and fall-related consequences such as fractures, further functional decline, immobility, and causal mortality (57). The agerelated decline in muscle mass seems to be primarily attributable to atrophy of type II muscle fibers, contributing to muscle weakness (58-60). It is commonly assumed that muscle strength and power are essential factors for coping with large balance disturbances. Nevertheless, muscle strength assessment and training usually only moderately correlate to basic balance tests and falls (61-63). To better understand the impact of muscle aging on mobility and balance control, chapter 6 focuses on the role of muscle fiber type composition in the ability to cope with large balance disturbances in young and older adults. Finally, in chapter 7 the findings of all the parts of this thesis are summarized and discussed in a broader perspective, and the overall conclusions are presented. 


\section{REFERENCES}

1. Lutz, W., Sanderson, W. \& Scherbov, S. The coming acceleration of global population ageing. Nature 451, 716-719 (2008).

2. Kinsella, K. Demographic Dimensions of Global Aging: Journal of Family Issues (2016). doi:10.1177/019251300021005002

3. United Nations. World Population Ageing 2019. Department of Economic and Social Affairs, Population Division (2020).

4. Chang, V. W., Alley, D. E. \& Dowd, J. B. Trends in the Relationship Between Obesity and Disability, 1988-2012. American Journal of Epidemiology 186, 688-695 (2017).

5. Coresh, J., Astor, B. C., Greene, T., Eknoyan, G. \& Levey, A. S. Prevalence of chronic kidney disease and decreased kidney function in the adult US population: Third National Health and Nutrition Examination Survey. Am J Kidney Dis 41, 1-12 (2003).

6. Driver, J. A., Djousse, L., Logroscino, G., Gaziano, J. M. \& Kurth, T. Incidence of cardiovascular disease and cancer in advanced age: prospective cohort study. BMJ 337, a2467-a2467 (2008).

7. Mitchell, W. K. et al. Sarcopenia, Dynapenia, and the Impact of Advancing Age on Human Skeletal Muscle Size and Strength; a Quantitative Review. Front. Physio. 3, (2012).

8. Zierer, J. et al. Exploring the molecular basis of age-related disease comorbidities using a multi-omics graphical model. Sci Rep 6, 37646 (2016).

9. Rechel, B. et al. Ageing in the European Union. The Lancet 381, 1312-1322 (2013).

10. Kanasi, E., Ayilavarapu, S. \& Jones, J. The aging population: demographics and the biology of aging. Periodontol 2000 72, 13-18 (2016).

11. Roubenoff, R. Sarcopenia and its implications for the elderly. Eur J Clin Nutr 54, S40-S47 (2000).

12. Janssen, I., Heymsfield, S. B. \& Ross, R. Low Relative Skeletal Muscle Mass (Sarcopenia) in Older Persons Is Associated with Functional Impairment and Physical Disability. Journal of the American Geriatrics Society 50, 889-896 (2002).

13. Zoico, E. et al. Physical disability and muscular strength in relation to obesity and different body composition indexes in a sample of healthy elderly women. Int $J$ Obes 28, 234-241 (2004).

14. Newman, A. B. et al. Long-Term Function in an Older Cohort-The Cardiovascular Health Study All Stars Study: CHS ALL STARS LONG-TERM FUNCTION. Journal of the American Geriatrics Society 57, 432-440 (2009).

15. Visser, M. et al. Muscle Mass, Muscle Strength, and Muscle Fat Infiltration as Predictors of Incident Mobility Limitations in Well-Functioning Older Persons. The Journals of Gerontology Series A: Biological Sciences and Medical Sciences 60, 324333 (2005).

16. Talbot, L. A., Musiol, R. J., Witham, E. K. \& Metter, E. J. Falls in young, middle-aged and older community dwelling adults: perceived cause, environmental factors and injury. BMC Public Health 5, 86 (2005).

17. Crescenzo, R. et al. Skeletal Muscle Mitochondrial Energetic Efficiency and Aging. IJMS 16, 10674-10685 (2015).

18. Arango-Lopera, V. E., Arroyo, P., Gutiérrez-Robledo, L. M., Perez-Zepeda, M. U. \& Cesari, M. Mortality as an adverse outcome of sarcopenia. J Nutr Health Aging 17, 259-262 (2013). 
19. Fulop, T. et al. Aging, frailty and age-related diseases. Biogerontology 11, 547-563 (2010).

20. Rodríguez-Mañas, L. et al. Searching for an Operational Definition of Frailty: A Delphi Method Based Consensus Statement. The Frailty Operative Definition-Consensus Conference Project. The Journals of Gerontology: Series A 68, 62-67 (2013).

21. Fedarko, N. S. The Biology of Aging and Frailty. Clinics in Geriatric Medicine 27, 27 37 (2011).

22. Bergström, J., Hermansen, L., Hultman, E. \& Saltin, B. Diet, Muscle Glycogen and Physical Performance. Acta Physiologica Scandinavica 71, 140-150 (1967).

23. Defronzo, R. A. Glucose Intolerance and Aging. 28, 7 (1979).

24. Shulman, G. I. et al. Quantitation of muscle glycogen synthesis in normal subjects and subjects with non-insulin-dependent diabetes by $13 \mathrm{C}$ nuclear magnetic resonance spectroscopy. N Engl J Med 322, 223-228 (1990).

25. Spinelli, R. et al. Molecular basis of ageing in chronic metabolic diseases. $J$ Endocrinol Invest 43, 1373-1389 (2020).

26. Umanskaya, A. et al. Genetically enhancing mitochondrial antioxidant activity improves muscle function in aging. Proc Natl Acad Sci USA 111, 15250-15255 (2014).

27. Joseph, A.-M. et al. Dysregulation of Mitochondrial Quality Control Processes Contribute to Sarcopenia in a Mouse Model of Premature Aging. PLoS ONE 8, e69327 (2013).

28. Mills, K. F. et al. Long-Term Administration of Nicotinamide Mononucleotide Mitigates Age-Associated Physiological Decline in Mice. Cell Metabolism 24, 795-806 (2016).

29. Cantó, C. et al. The NAD+ Precursor Nicotinamide Riboside Enhances Oxidative Metabolism and Protects against High-Fat Diet-Induced Obesity. Cell Metabolism 15, 838-847 (2012).

30. Ferree, A. \& Shirihai, O. Mitochondrial Dynamics: The Intersection of Form and Function. 28 (2018).

31. Gonzalez-Freire, M. et al. Reconsidering the Role of Mitochondria in Aging. GERONA 70, 1334-1342 (2015).

32. López-Otín, C., Blasco, M. A., Partridge, L., Serrano, M. \& Kroemer, G. The Hallmarks of Aging. Cell 153, 1194-1217 (2013).

33. Boffoli, D. et al. Decline with age of the respiratory chain activity in human skeletal muscle. Biochimica et Biophysica Acta (BBA) - Molecular Basis of Disease 1226, 73 82 (1994).

34. Short, K. R. et al. Decline in skeletal muscle mitochondrial function with aging in humans. Proceedings of the National Academy of Sciences 102, 5618-5623 (2005).

35. Chabi, B. et al. Mitochondrial function and apoptotic susceptibility in aging skeletal muscle. Aging Cell 7, 2-12 (2008).

36. Lagerwaard, B., Nieuwenhuizen, A. G., de Boer, V. C. J. \& Keijer, J. In vivo assessment of mitochondrial capacity using NIRS in locomotor muscles of young and elderly males with similar physical activity levels. GeroScience 42, 299-310 (2020).

37. Marzetti, E. et al. Mitochondrial dysfunction and sarcopenia of aging: From signaling pathways to clinical trials. The International Journal of Biochemistry \& Cell Biology 45, 2288-2301 (2013).

38. Joseph, A.-M. et al. The impact of aging on mitochondrial function and biogenesis pathways in skeletal muscle of sedentary high- and low-functioning elderly individuals: Mitochondrial function in elderly individuals. Aging Cell 11, 801-809 (2012). 
39. Coen, P. M. et al. Skeletal Muscle Mitochondrial Energetics Are Associated With Maximal Aerobic Capacity and Walking Speed in Older Adults. The Journals of Gerontology: Series A 68, 447-455 (2013).

40. Choi, S. et al. ${ }^{31} \mathrm{P}$ Magnetic Resonance Spectroscopy Assessment of Muscle Bioenergetics as a Predictor of Gait Speed in the Baltimore Longitudinal Study of Aging. GERONA 71, 1638-1645 (2016).

41. Zane, A. C. et al. Muscle strength mediates the relationship between mitochondrial energetics and walking performance. Aging Cell 16, 461-468 (2017).

42. Santanasto, A. J. et al. The relationship between mitochondrial function and walking performance in older adults with a wide range of physical function. Experimental Gerontology 81, 1-7 (2016).

43. Broskey, N. T. et al. Exercise efficiency relates with mitochondrial content and function in older adults. Physiol Rep 3, e12418 (2015).

44. Distefano, G. et al. Chronological Age Does not Influence Ex-vivo Mitochondrial Respiration and Quality Control in Skeletal Muscle. GERONA glw102 (2016). doi:10.1093/gerona/glw102

45. Larsen, S. et al. The influence of age and aerobic fitness: effects on mitochondrial respiration in skeletal muscle. Acta Physiol (Oxf) 205, 423-432 (2012).

46. Hallal, P. C. et al. Global physical activity levels: surveillance progress, pitfalls, and prospects. The Lancet 380, 247-257 (2012).

47. Koyanagi, A., Stubbs, B., Smith, L., Gardner, B. \& Vancampfort, D. Correlates of physical activity among community-dwelling adults aged 50 or over in six low- and middle-income countries. PLoS ONE 12, e0186992 (2017).

48. Cartee, G. D., Hepple, R. T., Bamman, M. M. \& Zierath, J. R. Exercise Promotes Healthy Aging of Skeletal Muscle. Cell Metabolism 23, 1034-1047 (2016).

49. Booth, F. W. Lack of exercise is a major cause of chronic diseases. 135 (2014).

50. Ruegsegger, G. N. \& Booth, F. W. Health Benefits of Exercise. 15

51. Romanello, V. \& Sandri, M. The connection between the dynamic remodeling of the mitochondrial network and the regulation of muscle mass. Cell. Mol. Life Sci. (2020). doi:10.1007/s00018-020-03662-0

52. Houtkooper, R. H. et al. The metabolic footprint of aging in mice. Sci Rep 1, 134 (2011).

53. Gao, A. W. et al. A sensitive mass spectrometry platform identifies metabolic changes of life history traits in C. elegans. Sci Rep 7, 2408 (2017).

54. Yu, Z. et al. Human serum metabolic profiles are age dependent: Metabolic profiles associated with age. Aging Cell 11, 960-967 (2012).

55. Lawton, K. A. et al. Analysis of the adult human plasma metabolome. Pharmacogenomics 9, 383-397 (2008).

56. Levine, M. E. Modeling the Rate of Senescence: Can Estimated Biological Age Predict Mortality More Accurately Than Chronological Age? The Journals of Gerontology Series A: Biological Sciences and Medical Sciences 68, 667-674 (2013).

57. Terroso, M., Rosa, N., Torres Marques, A. \& Simoes, R. Physical consequences of falls in the elderly: a literature review from 1995 to 2010. Eur Rev Aging Phys Act 11, 51-59 (2014).

58. Kramer, I. F. et al. Extensive Type II Muscle Fiber Atrophy in Elderly Female Hip Fracture Patients. The Journals of Gerontology: Series A 72, 1369-1375 (2017). 
59. McPhee, J. S. et al. The Contributions of Fiber Atrophy, Fiber Loss, In Situ Specific Force, and Voluntary Activation to Weakness in Sarcopenia. The Journals of Gerontology: Series A 73, 1287-1294 (2018).

60. St-Jean-Pelletier, F. et al. The impact of ageing, physical activity, and pre-frailty on skeletal muscle phenotype, mitochondrial content, and intramyocellular lipids in men: Muscle phenotype, mitochondrial mass, and lipid content in human ageing. Journal of Cachexia, Sarcopenia and Muscle 8, 213-228 (2017).

61. Orr, R., Raymond, J. \& Fiatarone Singh, M. Efficacy of Progressive Resistance Training on Balance Performance in Older Adults: A Systematic Review of Randomized Controlled Trials. Sports Medicine 38, 317-343 (2008).

62. Horlings, C. G. C., van Engelen, B. G. M., Allum, J. H. J. \& Bloem, B. R. A weak balance: the contribution of muscle weakness to postural instability and falls. Nat Clin Pract Neurol 4, 504-515 (2008).

63. Sherrington, C. et al. Exercise for preventing falls in older people living in the community. Cochrane Database Syst Rev 1, CD012424 (2019). 


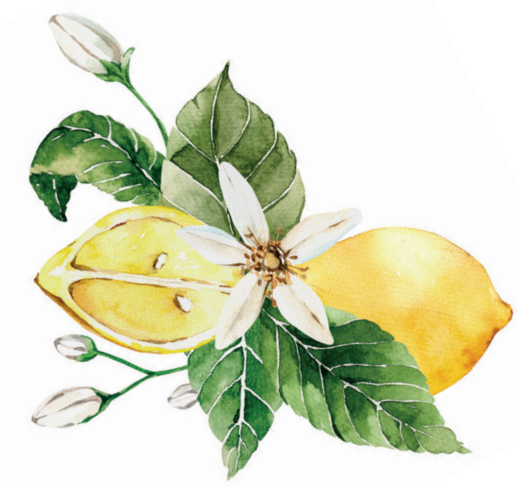

\section{CHAPTER 2}

\section{Skeletal muscle mitochondrial network dynamics in metabolic disorders and ageing.}

Ciarán E. Fealy, Lotte Grevendonk, Joris Hoeks, Matthijs Hesselink

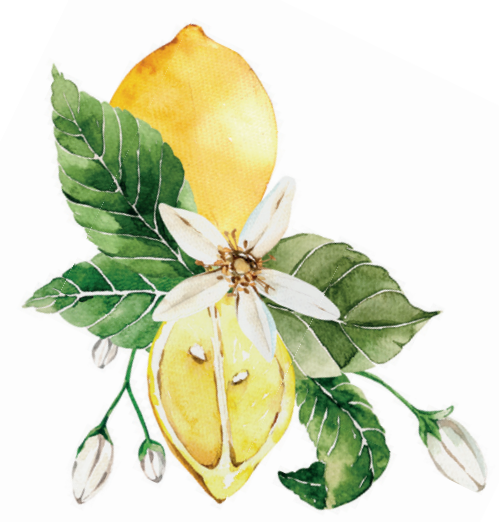




\section{Chapter 2}

\section{ABSTRACT}

With global demographics trending towards an ageing population, the numbers of individuals with an age-associated loss of independence is increasing. A key contributing factor is loss of skeletal muscle mitochondrial, metabolic, and contractile function. Recent advances in imaging technologies have demonstrated the importance of mitochondrial morphology and dynamics in the pathogenesis of disease. In this review, we examine the evidence for altered mitochondrial dynamics as a mechanism in age and obesity-associated loss of skeletal muscle function, with a particular focus on the available human data. We highlight some of the areas where more data are needed to identify the specific mechanisms connecting mitochondrial morphology and skeletal muscle dysfunction.

Keywords: Metabolic disease, sarcopenia, ageing, mitochondria, mitochondrial dynamics 


\section{Skeletal muscle in age and age-associated diseases}

Increases in human longevity illustrate the remarkable advances achieved in biomedicine, nutrition and healthcare over the past century. Yet as lifespan has increased, gains in healthspan - defined as the period of life spent in good health, free from the chronic diseases and disabilities of aging [1] - have stagnated [2]. With the proportion of elderly among the global population set to reach $>20 \%$ in the next decades [3], the imperative to find new solutions to the problem of age-associated loss of independence has become increasingly relevant.

One of the main obstacles to improvements in healthspan is the contemporaneous rise in overweight and obesity in the global population. Although overweight and obesity have been associated with an increase in premature death, the impact of these on mortality has declined such that recent evidence suggests that elderly individuals with overweight or mild obesity now have a life expectancy similar to normal weight elderly [4]. However, this group is at increased risk of disability and spend more years with limitations in physical function compared to normal weight elderly $[5,6]$.

Deterioration in skeletal muscle mass, metabolic fitness, and contractile vigour are fundamental to the progression of both metabolic disease and age-associated loss of independence (Figure 1). Increased adiposity and ectopic lipid deposition, whether in the presence of obesity or not, contribute to skeletal muscle insulin resistance [7], are common features of ageing [8-10], and strong predictors of both metabolic disease progression [11, 12] and muscle strength and mass [9, 10]. Increasing adiposity blunts the effectiveness of insulin and amino acid stimulation of muscle protein synthesis [13]. Muscle, the primary target tissue for insulin stimulated glucose disposal, is a key regulator of whole-body glucose homeostasis. As such, reduced muscle mass is also associated with reduced energy expenditure [14]. Age-related decline in both physical function and metabolic health can be mitigated, in large part, by increased physical activity levels and exercise training. Thus, the underlying pathology is one of adaptation to chronic environmental stimuli and allostatic loading rather than an obligatory consequence of ageing $[7,8]$. Progressive deterioration in mitochondrial capacity has been linked to age- and obesity- associated muscle defects though there remains little consensus on a specific mechanistic pathway to 
explain this association. Recent developments in understanding the dynamic behaviour of mitochondrial networks and quality control pathways have opened up new prospective avenues of mitochondrial involvement in physical and metabolic decline. In this review we will examine the evidence for the presence of aberrant mitochondrial network dynamics in the pathogenesis of age-associated skeletal muscle dysfunction, with a focus, where available, on evidence from human research.

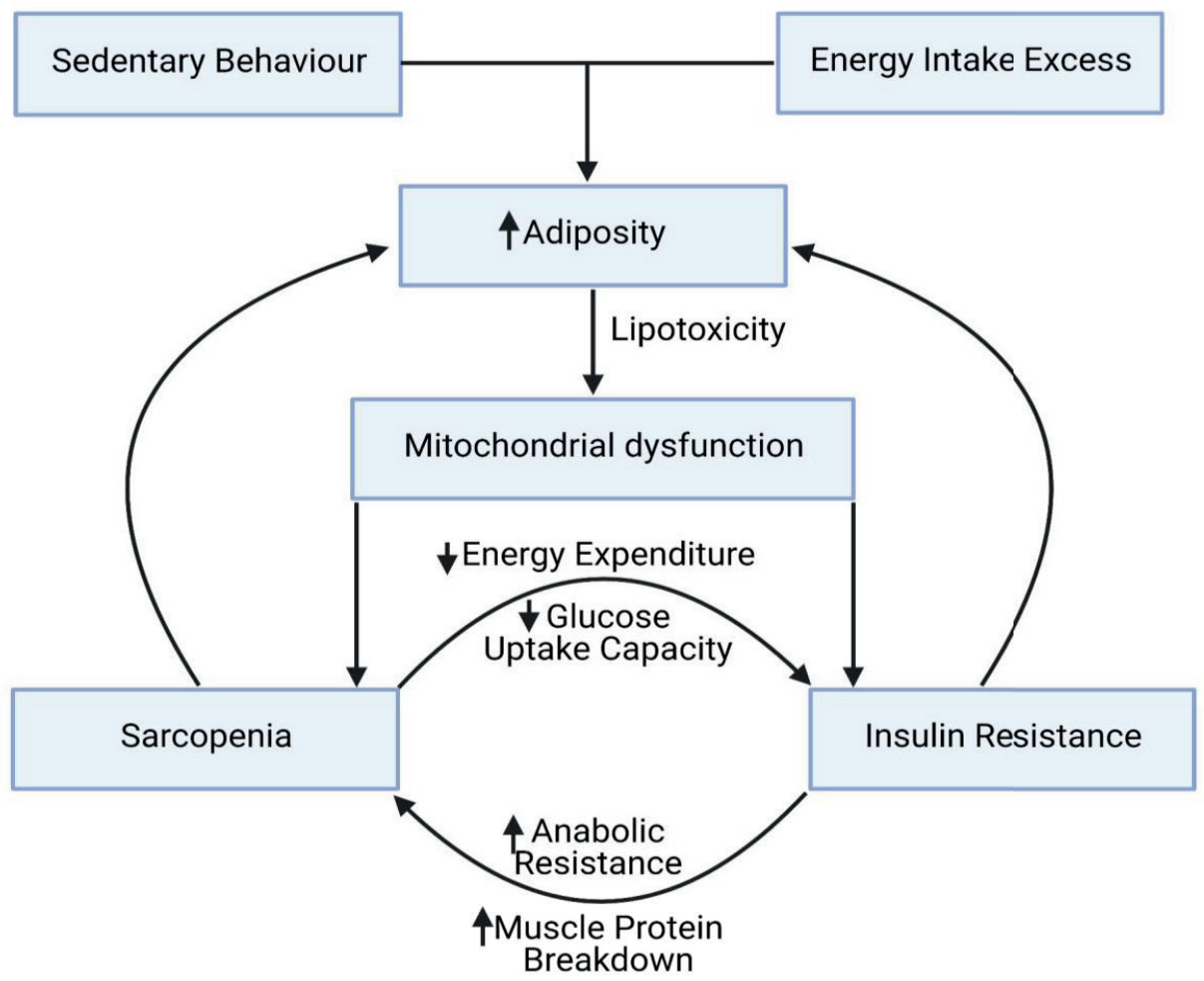

Figure 1: Reciprocal relation between muscle mass and metabolic homeostasis. Both a sedentary behaviour and an excess energy intake during aging negatively influence body composition by increasing fat mass. Excess adiposity is associated with increased ectopic lipid deposition leading to lipotoxicity and consequent mitochondrial dysfunction. Mitochondrial dysfunction has been implicated in the onset of sarcopenia and insulin resistance. Sarcopenia and insulin resistance likely interact whereby loss of muscle mass results in reduced energy expenditure and reduced capacity for glucose uptake while insulin resistance may induce anabolic resistance to insulin and amino acids and reduce insulin inhibition of muscle protein breakdown. Collectively these mechanisms further exacerbate adiposity in a positive feedback loop resulting a progressive decline in metabolic and functional fitness in aged muscle. 


\section{Skeletal muscle mitochondria are an important target for increasing healthspan}

One of the early consequences of chronic lipotoxicity (see Glossary) in both metabolic disease and ageing is a reduction in mitochondrial content and capacity [9]. However, the extent to which reduced mitochondrial capacity contributes to impaired muscle function in obesity and ageing is debated. Classically, mitochondrial function has been viewed through the prism of mitochondrial density and intrinsic bioenergetic capacity, however fresh insights into the varied functions of mitochondria necessitates an expansion of the concept. In the present view, mitochondrial function also includes the ability of mitochondria to move and physically interact with each other in dynamic reticular networks. Mitochondrial network architecture, regulated by the processes of fusion and fission (Figure 2), varies widely among cell types, and morphology is intrinsically linked to the energetic and functional aspects of a cell. Thus, in dividing cells, and in cells with a high mitochondrial turnover, mitochondrial fission predominates and morphology shifts towards a "fragmented" network [15]. On the other hand, skeletal muscle which requires rapid conductance of energy potentials forms a vast interconnected reticulum that acts as a power grid to distribute membrane potential according to energetic requirements $[16,17,18]$, though the degree of networking may be less apparent in humans compared to mice [16]. Mitochondrial morphology and dynamics have been linked to a variety of cellular processes including cell division [19], regulation of gene expression [20], apoptosis [14, 21] and autophagy [22], removal of damaged mitochondria (mitophagy) [23], calcium homeostasis [24], production of reactive oxygen species (ROS) [25], and regulation of cellular bioenergetic capacity and efficiency [26]. Mitochondrial morphology also responds to the nutrient status of cells with fragmentation observed in conditions of high nutrient availability and fusion predominating to maintain higher bioenergetic efficiency where nutrients are scarce [27]. The major proteins that regulate mitochondrial morphology are described in Table 1. Given the ubiquity of cellular functions in which mitochondrial dynamics participate it is hardly surprising that dysregulation of mitochondrial network dynamics has been implicated in an increasing number of disease states $[23,28]$, including metabolic disease $[25,26]$ and age-associated functional decline [29]. 


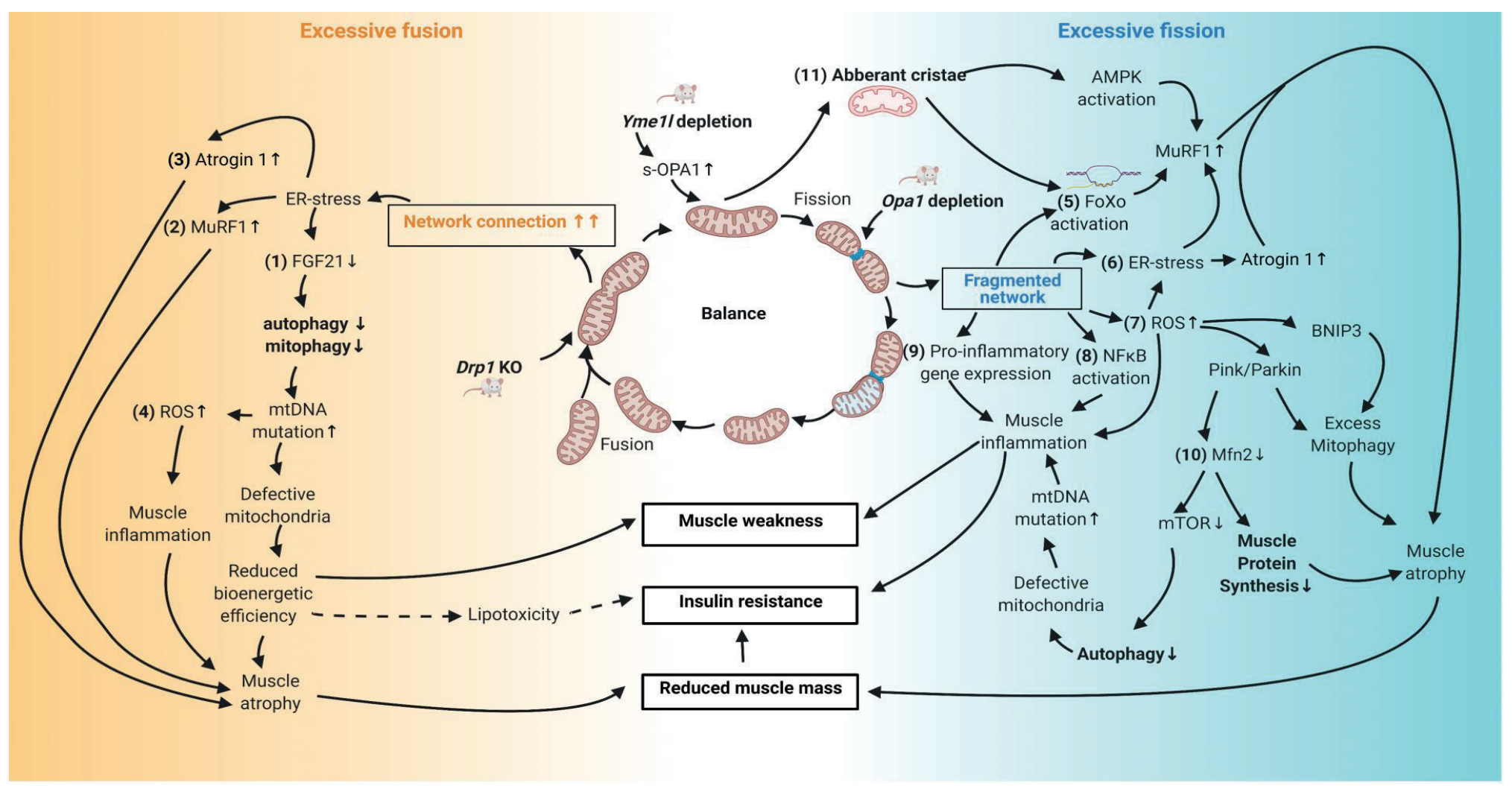

Figure 2: Putative pathways in response to dysregulated mitochondrial dynamics in age-related decline in muscle function and metabolism 
Figure 2: Putative pathways in response to dysregulated mitochondrial dynamics in age-related decline in muscle function and metabolism Depicted are some of the identified putative pathways which may explain the contribution of alteration in mitochondrial morphology to the decline in metabolic and functional fitness in aged muscle. Drp1 knockout animals represent a model of excessive fusion. Excessive fusion leads to the ER-stress mediated downregulation of FGF21 (1). Lower levels of FGF21 result in reduced mitophagy and autophagy, which result in excessive levels of mitochondrial DNA mutations, leading to inefficient mitochondria, a reduced bioenergetic efficiency, and eventually muscle weakness and a higher risk for lipotoxicty-induced insulin resistance. ER-stress also induces the expression of the atrogenic genes and Atrogin 1 (2) and MuRF1 (2), which, together with the mtDNA damage induced ROS-mediated (4) muscle inflammation, enhances muscle atrophy also resulting in a reduced muscle mass and consequent insulin resistance. Opa1 depletion represents a model for excessive fission and a fragmented network. The fragmented network results in activation of the atrogenic forkhead box O's (FOXOs) (5), increased stress on the ER (6), increased ROS (7), NfkB activation (8), and increased expression of several pro-inflammatory genes (9). ER-stress (6) leads to upregulation of MuRF1 expression and both the activation of FOXO (5) and increased ER-stress (6) upregulates the expression of MuRF1 and Atrogin 1, leading to muscle atrophy mediated decline in muscle mass and consequent insulin resistance. ROS (7) additionally mediates the upregulation of BNIP3 and Parkin which both result in excessive mitophagy and muscle atrophy. The ROS-mediated (7) activation of Parkin also leads to a decline in Mfn2 (10), which impairs muscle protein synthesis, resulting in muscle atrophy, a reduced muscle mass and consequent insulin resistance. Also, a decline in Mfn2 (10) leads to a decline in mTOR and consequently a decline in autophagy, resulting in defective mitochondria, increased mtDNA mutation and muscle inflammation. In parallel, increased ROS production (7), NFKB activation (8), and increased expression of proinflammatory genes (9) stimulate muscle tissue inflammation, together resulting in muscle weakness and insulin resistance. Yme1l depletion stimulates the expression of the S-Opa1, leading to aberrant mitochondrial cristae (11), which activates AMPK and FOXO (5), both stimulating the expression of MuRF1 and Atrogin 1 and leading to an atrogenic mediated reduction in muscle mass and increased insulin resistance. Both excessive fusion and excessive fission can impair muscle function and metabolic health. Abbreviations: Drp1 $=$ Dynamin related protein 1; ER $=$ endoplasmic reticulum; FGF21 = Fibroblast growth factor 21; DNA = deoxyribonucleic acid; MuRF1 = muscle RING-finger protein-1; mtDNA = mitochondrial deoxyribonucleic acid; ROS = reactive oxygen species; Opa1 = Optic atrophy 1; FOXOs = forkhead box O's ; NFKB = nuclear factor kappa-lightchain-enhancer of activated B cells; BNIP3 = BCL2 Interacting Protein 3; Mfn2 = Mitofusin2; YME1I = ATP-dependent zinc metalloprotease YME1L; S-Opa1 = Optic atrophy 1 (short form); $A M P K=$ adenosine monophosphate-activated protein kinase. 
Table 1: Major fusion and fission proteins that regulate mitochondrial morphology

\begin{tabular}{|c|c|c|c|c|}
\hline Protein & & Location & Function & \\
\hline \multicolumn{5}{|l|}{ Fusion proteins } \\
\hline Mfn1 & Mitofusin-1 & $\begin{array}{l}\text { Outer } \\
\text { mitochondrial } \\
\text { membrane }\end{array}$ & $\begin{array}{l}\text { Outer mitochondrial membrane } \\
\text { fusion }\end{array}$ & [93] \\
\hline Mfn2 & Mitofusin-2 & $\begin{array}{l}\text {-Outer } \\
\text { mitochondrial } \\
\text { membrane } \\
\text {-Endoplasmic } \\
\text { reticulum }\end{array}$ & $\begin{array}{l}\text { Outer mitochondrial membrane } \\
\text { fusion } \\
\text {-Facilitating mitochondrial calcium } \\
\text { influx from ER } \\
\text {-ER-Mitochondria contact site } \\
\text { tethering } \\
\text { Facilitates mitophagy as a Parkin } \\
\text { receptor }\end{array}$ & $\begin{array}{l}{[94]} \\
{[95]} \\
{[96]} \\
{[97]}\end{array}$ \\
\hline $\begin{array}{l}\text { L-Opa1 } \\
\text { (long } \\
\text { form) }\end{array}$ & Optic atrophy 1 & $\begin{array}{l}\text { Inner } \\
\text { mitochondrial } \\
\text { membrane }\end{array}$ & $\begin{array}{l}\text { Inner mitochondrial membrane } \\
\text { fusion }\end{array}$ & \multirow{4}{*}{ [98] } \\
\hline $\begin{array}{l}\text { S-Opa1 } \\
\text { (short } \\
\text { form) }\end{array}$ & Optic atrophy 1 & $\begin{array}{l}\text { Mitochondrial } \\
\text { intermembrane } \\
\text { space }\end{array}$ & $\begin{array}{l}\text { Cristae morphogenesis (together } \\
\text { with L-Opa1) }\end{array}$ & \\
\hline YME1L & $\begin{array}{l}\text { ATP-dependent } \\
\text { zinc } \\
\text { metalloprotease } \\
\text { YME1L }\end{array}$ & $\begin{array}{l}\text { Inner } \\
\text { mitochondrial } \\
\text { membrane }\end{array}$ & Cleavage of L-Opa1 into S-Opa1 & \\
\hline Oma1 & $\begin{array}{l}\text { Zinc } \\
\text { metallopeptidase }\end{array}$ & $\begin{array}{l}\text { Inner } \\
\text { mitochondrial } \\
\text { membrane }\end{array}$ & $\begin{array}{l}\text { Cleavage of L-Opa1 during } \\
\text { mitochondrial depolarization }\end{array}$ & \\
\hline \multicolumn{5}{|l|}{ Fission proteins } \\
\hline Drp1 & $\begin{array}{l}\text { Dynamin related } \\
\text { protein } 1\end{array}$ & $\begin{array}{l}\text { Outer } \\
\text { mitochondrial } \\
\text { membrane }\end{array}$ & Mitochondrial fission & \multirow{5}{*}{ [99] } \\
\hline Fis 1 & $\begin{array}{l}\text { Mitochondrial } \\
\text { fission } 1\end{array}$ & $\begin{array}{l}\text { Outer } \\
\text { mitochondrial } \\
\text { membrane }\end{array}$ & $\begin{array}{l}\text { Putative Drp1 receptor; may } \\
\text { facilitate Drp1-mediated } \\
\text { mitochondrial fission }\end{array}$ & \\
\hline Mff & $\begin{array}{l}\text { Mitochondrial } \\
\text { fission factor }\end{array}$ & $\begin{array}{l}\text { Outer } \\
\text { mitochondrial } \\
\text { membrane }\end{array}$ & $\begin{array}{l}\text { Mitochondrial receptor for Drp1; } \\
\text { facilitates Drp1-mediated } \\
\text { mitochondrial fission }\end{array}$ & \\
\hline MiD49 & $\begin{array}{l}\text { Mitochondrial } \\
\text { dynamics protein } \\
\text { of } 49 \mathrm{kDa}\end{array}$ & $\begin{array}{l}\text { Outer } \\
\text { mitochondrial } \\
\text { membrane }\end{array}$ & $\begin{array}{l}\text { Mitochondrial receptor for Drp1; } \\
\text { may regulate the DRP1 and } \\
\text { maintain its inactive state until } \\
\text { fission is required }\end{array}$ & \\
\hline MiD51 & $\begin{array}{l}\text { Mitochondrial } \\
\text { dynamics protein } \\
\text { of } 51 \mathrm{kDa}\end{array}$ & $\begin{array}{l}\text { Outer } \\
\text { mitochondrial } \\
\text { membrane }\end{array}$ & $\begin{array}{l}\text { Mitochondrial receptor for Drp1; } \\
\text { facilitates Drp1-mediated } \\
\text { mitochondrial fission } \\
\text { (Fis } 1 \text { and Mff independent) }\end{array}$ & \\
\hline
\end{tabular}




\section{Mitochondrial network morphology in metabolic disorders}

There is a growing consensus that obesity and metabolic disease are associated with mitochondrial network fragmentation in skeletal muscle. Increased mitochondrial network fragmentation was first demonstrated in skeletal muscle of Zucker Diabetic Fatty rats [30]. Similarly, obese mice that are deficient for leptin receptors and mice with high fat diet (HFD)-induced obesity were found to exhibit smaller and shorter mitochondria in skeletal muscle compared to lean mice [31]. In line with these findings, reduced propagation of mitochondrial matrix-targeted photoactivatable green fluorescent protein was observed in skeletal muscle of mice fed a high fat diet compared to chow fed controls [32], indicating a reduction in the degree of mitochondrial networking. Importantly, the presence of increased mitochondrial fragmentation in skeletal muscle has also been observed in humans with obesity and type 2 diabetes (T2D) compared to a lean reference group. This morphological phenotype was restored 1.5-years after bariatric surgery [33]. Similarly, mitochondrial capacity in patients undergoing laparoscopic gastric banding surgery was normalized 1-year post-surgery, which was independent of changes in mitochondrial content [34]. Taken together, these studies suggest an interdependency of mitochondrial form and function in obese patients. In young healthy humans, induction of insulin resistance by lipid infusion resulted in skeletal muscle mitochondrial fragmentation in association with activation of DRP1 by phosphorylation at serine 616 (Drp1 ${ }^{\text {ser616}}$ ) [35]. Strikingly, this effect was observed without reduction in mitochondrial capacity or content, which suggests that functional defects may be secondary to chronic lipotoxicity. Primary myotubes cultured from skeletal muscle biopsies obtained from severely obese non-diabetic and T2D participants retained a more fragmented mitochondrial network, relative to young lean healthy individuals [36]. This change in mitochondrial network was associated with increased Drp1 ${ }^{\text {ser616. }}$. Moreover, the number of fragmented mitochondria in cultured myotubes from the same population was reduced at 7 months after Rouxen-Y gastric bypass (RYGB) [37] concomitant with reductions in Drp1 ${ }^{\text {ser616. The }}$ retention of these morphological characteristics in primary myotubes despite identical cell culture conditions suggests the involvement of transcriptional programming and is therefore not solely an acute response to a positive energy balance and lipotoxicity. 


\section{Regulation of skeletal muscle mitochondrial morphology in metabolic disorders}

\section{Mitochondrial Fusion}

Protein expression studies also provide insights into the role of mitochondrial dynamics in metabolic disorders. Several lines of evidence support negative transcriptional regulation of mitochondrial fusion as an underlying component of mitochondrial fragmentation in metabolic disease. Reduced mitofusin-2 (MFN2) mRNA and protein expression is observed in skeletal muscle in obese rodents relative to lean controls [30, 32, 38, 39] and also in humans [30, 40, 41]. Repression of MFN2 in a rat muscle cell line decreased pyruvate and palmitate oxidation [30], increased production of ROS, and increased c-Jun N-terminal kinases (JNK) and nuclear factor kappa-light-chain-enhancer of activated B cells (NFKB) signaling in association with reduced glucose uptake and insulin signaling (Figure 2). Conversely, MFN2 gain of function reduced ROS production and restored insulin signaling [42, 43]. Similarly, in humans MFN2 mRNA correlated with glucose oxidation and increases in mRNA levels following bariatric surgery were positively correlated with the percentage change in glucose oxidation between fasted and insulin stimulated conditions during a hyperinsulinaemic-euglycaemic clamp procedure [40]. In healthy premenopausal women, MFN2 protein expression was associated with ROS emitting capacity in permeabilized skeletal muscle fibers [44]. Expression of the inner mitochondrial membrane fusion protein Optic Atrophy-1 (OPA1), along with the mitochondrial fission protein FIS1, was highest in athletes when compared to individuals with type 2 diabetes [45]. Enhanced mitochondrial networking was also apparent in the athletes [45] perhaps indicating that athletes exhibit enhanced mitochondrial turnover through both mitochondrial fusion and fission, although with fusion the predominant factor in the resting homeostatic state. Provision of a eucaloric diet along with aerobic exercise training in older obese individuals induced a significant weight loss and resulted in increased skeletal muscle Optic Atrophy-1 (OPA1) [46]. This further supports a pro-fusion mitochondrial phenotype in response to exercise and a negative energy balance. In addition, as in a rat model [47], RYGB surgery in obese patients increased skeletal muscle MFN2 gene expression at 3 - and 12 months post-surgery in obese individuals [48]. However, in contrast to above findings, several studies reported that skeletal muscle 
mitochondrial fusion gene and/or protein expression were not altered in human obesity [49] or T2D [50]. Moreover, single fiber analysis of skeletal muscle in morbid obesity revealed that the amount of both MFN1/2 was in fact higher in muscle fibers that presented fragmented networks [33]. An alternative explanation for these discrepancies may relate to the non-canonical functions of MFN2 such as involvement in lipid droplet [51] and Sarcoplasmic reticulum (SR)- mitochondria contact sites [52]. In particular, dysregulated SR-mitochondria interactions have been implicated in metabolic disease in animals and humans [51, 53, 54]. Moreover, a recent study [55] demonstrated that in healthy individuals fat oxidation was affected by both mitochondrial size and SR-mitochondria contact sites. It is therefore worth acknowledging that alterations in fusion proteins may also influence metabolic disease independent of their effects on mitochondrial morphology.

\section{Mitochondrial Fission}

Besides reduced fusion, excessive mitochondrial fission has also been implicated in skeletal muscle metabolic dysfunction. Similar to humans [35], in C2C12 cells and rodent muscle excess lipids resulted in mitochondrial fragmentation and reduced glucose uptake [31]. This was attenuated by inhibition of DRP1 [31]. Conversely, exercise training in obese humans results in a reduction in DRP1 activation which was correlated to improvements in insulin sensitivity and fat oxidation [56]. Along the same lines, expression of FIS1 was elevated in muscle of obese and T2D humans, and reduced by diet and exercise, and RYGB. DRP1-mediated mitochondrial fragmentation has also been implicated in ceramide-induced $\mathrm{H}_{2} \mathrm{O}_{2}$ production, impaired mitochondrial bioenergetics, and insulin resistance in C2C12 myotubes [57]. Recent evidence from liver cells support a role for DRP1 adaptor protein MFF in mediating ceramide-induced metabolic dysfunction [58]. Ceramide species, particularly C16:0 ceramides, are a major lipotoxic lipid class and are thought to be significant mediators of insulin resistance [59]. These data further support a link between lipotoxicity and mitochondrial fragmentation in skeletal muscle metabolic dysfunction observed in metabolic disorders.

Obviously, mitochondrial morphology is defined by a balance between the rates of fusion and fission and it may be that mitochondrial morphology per se modulates substrate metabolism and insulin sensitivity regardless of whether shifts in 
morphology are driven by alterations in either fission or fusion. Development of biomarkers of mitochondrial morphology in bigger datasets encompassing healthy and obese participants is therefore essential to advance our understanding of the role of mitochondrial dynamics in metabolic disorders. In this context, focusing on the ratio between fusion and fission protein expression rather than the individual proteins, may prove to be a useful strategy. Several attempts to this effect have been made such as OPA1-FIS1 [46] or MFN2-FIS1 [60], however further validation may be necessary. Nonetheless, imaging remains the gold standard for determining mitochondrial morphology, and advances in imaging techniques have been instrumental in enhancing our understanding of mitochondrial networks in skeletal muscle. Mitochondria in skeletal muscle observed by electron microscopy appear in tightly packed clusters, with many mitochondria grouped together in subsarcollemal regions and appearing in doublets in the intermyofibrillar regions of the muscle. 3D imaging of rodent muscle, and more recently human muscle, indicate that skeletal muscle relies on the formation of nanotunnels between mitochondria for the efficient transmission of membrane potential and transfer of mitochondrial DNA (mtDNA) and proteins[16]. Nanotunneling is reduced in obese mice [32], but data in skeletal muscle of obese humans is lacking. Nonetheless, this discovery represents an additional challenge to defining the relationship between mitochondrial dynamics and mitochondrial morphology in human disease as the finding of increased fragmentation, despite increased mitochondrial nanotunneling, observed in humans with primary mtDNA mutations illustrates [16].

\section{Aberrant mitochondrial network morphology is a hallmark of skeletal muscle myopathy}

Mitochondrial morphology has long been associated with myopathy [61]. Indeed, abnormalities in muscle mitochondrial structure became a diagnostic factor for inherited skeletal muscle myopathies [62] in the latter third of the $20^{\text {th }}$ century. Whilst it is beyond the scope of the current review to provide an comprehensive overview of inherited and developed myopathies with a mitochondrial defect, it is nonetheless instructive to highlight some instances which illustrate a clear link between abberant mitochondrial morphology and skeletal muscle myopathy. In humans, mutations in the MFN2 gene result in Charcot-Marie-Tooth type 2A, a neuropathic disorder which is characterized by progressive muscle atrophy [63]. Autosomal dominant optic 
atrophy (ADOA) mutations in the OPA1 gene results in muscle wasting associated with reductions in ATP generating capacity and increased mtDNA mutations in skeletal muscle [64]. More recently a novel myopathy caused by a homozygous nonsense mutation in the MIEF2 gene encoding the mitochondrial dynamics protein of $49 \mathrm{kDa}$ (MID49) was associated with increased fusion, mitochondrial elongation, and aberrant cristae structure [65]. In cancer cachexia and chemotherapy-induced cachexia, DRP1, OPA1, and MFN2 gene expression were dramatically suppressed $[66,67]$.

\section{Mitochondrial Morphology in Ageing Muscle}

Sarcopenia is the age-associated loss of muscle mass and strength. In ageing, muscle mass begins to decline as early as age 40 at a rate of up to $1 \%$ per year while strength declines even more rapidly [68]. In aged muscle, reports from rodent studies suggest that aged mitochondria are smaller in size, while others suggest an increase in mitochondrial networking. Moreover, aged skeletal muscle displays impaired mitochondrial energetics [69] and increased mitochondrial-mediated apoptosis [70]. However, 2D imaging of human skeletal muscle has identified the presence of giant mitochondria in aged muscle indicating that there may be multiple mechanisms at play in the regulation of mitochondrial morphology in aged skeletal muscle. Interestingly, giant mitochondria in cultured myoblasts display both reduced levels of OPA1 and impaired capacity in fusion and autophagy [71]. In addition, muscle of aged mice also displayed abnormal mitochondrial structure and cristae formation (Figure 2) [72, 73]. These morphological alterations associate with reduced muscle cross-sectional area and reduced grip strength concomitant with reduced levels of OPA1 and DRP1 protein expression [73]. Consistent with this finding, grip strength is reduced in mice with a muscle-specific OPA knockout [74]. The discrepancies in mitochondrial morphologies reported in studies of ageing may relate to non-linear changes in fission and fusion over the lifespan [75]. These morphological alterations correlated with changes in muscle function evidenced at the different stages of mice life. Taken together these data illustrate a potential causal relationship between mitochondrial morphology and age-associated diseases of muscle weakness. 


\section{Mitochondrial dynamics and age-associated sarcopenia}

Several studies have indicated similar mitofusin gene [76] and protein [10, 77, 78] expression in skeletal muscle biopsies of young versus old volunteers. In addition, skeletal muscle OPA1 expression was unchanged [10] or decreased [77, 79] in elderly skeletal muscle, however the degree to which these elderly participants were sarcopenic is not apparent. In a recent study [80], MFN2 protein expression was lower in elderly patients with sarcopenia relative to non-sarcopenic elderly. This suggests that clearer characterization of sarcopenia status, or long term prospective studies in elderly, may be needed to determine the degree to which altered mitochondrial fusion is related to age-associated physical decline in humans. In spite of this, data from pre-clinical studies provide robust support for a link between altered mitochondrial fusion and age-related decline in muscle health. A reduction in both MFN1 and MFN2 has been demonstrated in aged mice, while global Mfn2 knockout in mice resulted in mitochondrial dysfunction, increased oxidative stress, a reduction in ambulatory activity, and reduced muscle cross-sectional area. Notably Mfn2 knockout in mice resulted in muscle atrophy by downregulation of protein synthesis via the mTOR pathway and an accumulation of autophagosomes (Figure 2) [81]. This is indicative of a reduction in autophagy, the cellular mechanism that maintains tissue quality by removal of damaged cellular components. Indeed, reduced autophagy is a common feature of muscle atrophy [82, 83] and ageing [84], and has been linked to reduced MFN2 in muscle cells [85]. MFN2 repression was also associated with the accumulation of defective mitochondria despite increased mitophagy signaling via ROS-dependent upregulation of BNIP3 and Parkin (Figure 2) [81]. Interestingly, Parkin mediated ubiquitination and degradation of MFN2 also induces muscle disuse atrophy through excessive mitochondrial fragmentation and mitophagy (Figure 2). This can be rescued by increasing mitochondrial mass through overexpression of the mitochondrial biogenesis activator Peroxisome proliferator-activated receptor gamma coactivator 1-alpha (PGC-1 $\alpha$ ) [86].

Opa1 depletion leads to reduced grip strength and muscle atrophy in rodents via elevated production of ROS [87], enhanced expression of pro-inflammatory genes [87], activation of the atrogenic forkhead box O's (FOXOs) class of transcription factors [88], and increased muscle inflammation characterized by NFKB activation (Figure 2). Moreover, depletion of the OPA1 cleavage factor ATP-dependent zinc 
metalloprotease YME1L (YME1L) in myotubes results in accumulation of the OPA1 short form and the activation of AMP-activated protein kinase (AMPK) and FOXO3, thereby leading to increased muscle RING-finger protein-1 (MuRF1) (Figure 2) [88]. Reduced OPA1 has also been implicated in skeletal muscle atrophy via both ERstress mediated translational inhibition and upregulation of atrogenic genes such as MuRF1, Atrogin 1 and Fibroblast growth factor 21 (FGF21) in rodent models (Figure 2) [79]. Collectively, these data illustrate how down-regulation of pro-fusion proteins in skeletal muscle can activate catabolic systems and ultimately cause muscle mass loss. However, whether these findings translate to age-associated declines in muscle protein synthesis and sarcopenia in human ageing remains an open question.

The evidence supporting increased mitochondrial fission in skeletal muscle sarcopenia in humans is limited, with studies showing similar protein expression of DRP1 and FIS1 in young and old sedentary adults [10, 77] and following 10 days of bed rest in older adults [89]. Nonetheless, there is evidence for both knockout and overexpression of Drp1 resulting in muscle atrophy, albeit through activation of separate atrogenic pathways (Figure 2) [90]. Intriguingly, muscle-specific Opa1 and Drp1 DKO mice had a less severe phenotype than Opa1 knockout mice, despite persistent mitochondrial dysfunction. This effect was primarily through a blunted atrogenic transcriptional activity and reduced FGF21, though at the cost of translational inhibition via increased ER stress and reduced autophagy and mitophagy (Figure 2) [90]. In contrast, data in mouse cardiomyocytes [91] and C. elegans suggests that co-deletion of fission and fusion protein expression results in decreases in lifespan and metabolic health. Nonetheless, these data highlight the need to maintain the appropriate balance between fission and fusion for preservation of skeletal muscle mass and function. Interestingly, muscle cells incubated with palmitate lose skeletal muscle integrity via modulation of mitochondrial fission gene expression, pro-inflammatory cytokines, and atrogenes, yet this effect was attenuated by co-incubation with oleate [92]. This suggests that the fatty acidmediated mitochondrial redox state is a significant factor in the regulation of myotube atrophy. This finding is consistent with the effects of lipids on skeletal muscle mitochondrial fragmentation in insulin resistance in cells and animals [31] and humans [35] and therefore highlights the potential interconnectedness between 


\section{Chapter 2}

muscle mitochondrial dynamics in both skeletal muscle metabolic function and quality.

\section{Concluding Remarks}

There is growing evidence for impaired mitochondrial network dynamics in the aetiology of skeletal muscle health in metabolic disorders and ageing. Yet what this review highlights is that there remains a scarcity of detailed mechanistic studies, particularly in human models. Such studies will be essential for understanding the role of altered mitochondrial networks in the pathogenesis of muscle dysfunction and for developing new therapeutics to target mitochondrial dynamics in human disease. Nonetheless, new technologies that have emerged including advances in imaging technology with increasing application to human tissue have shed new light on the organization of mitochondrial networks in muscle; these approaches open the possibility for deeper insights into the role of skeletal muscle mitochondrial dynamics in metabolic disorders and ageing. 


\section{GLOSSARY}

Lipotoxicity: The deleterious effects of lipid accumulation in non-adipose tissue. Mitochondrial Fusion: The physical merging of the outer and then the inner mitochondrial membranes of two originally distinct mitochondria.

Mitochondrial Fission: The division of a single mitochondria into two or more independent structures.

Apoptosis: Programmed cell death

Autophagy (macroautophagy): The process in which cellular contents are degraded by lysosomes or vacuoles and recycled.

Mitophagy: The selective degradation of mitochondria by autophagy.

Reactive oxygen species (ROS): are oxygen-containing radicals such as superoxide anion (O2-), hydrogen peroxide ( $\mathrm{H} 2 \mathrm{O} 2)$, and hydroxyl radical $(\mathrm{HO} \bullet)$ that can be generated by aerobic metabolism. ROS may serve as cell signaling molecules for normal biologic processes, however excessive production of ROS can result in damage to multiple cellular organelles and processes.

Type 2 diabetes (T2D): Type 2 diabetes mellitus is a metabolic disorder that results in hyperglycaemia due to reduced effectiveness of the hormone insulin (insulin resistance) and an inability of the pancreas to produce enough insulin to over come insulin resistance.

Bariatric surgery: covers a variety of weight loss surgeries including

Laparoscopic gastric banding surgery: is a surgical procedure that involves the placement of an adjustable belt around the upper portion of the stomach using a laparascope. The belt limits the expansion of the stomach conferring increased satiety in the patient.

Roux-en-Y gastric bypass (RYGB): is a surgical procedure that involves creating a small gastric pouch connected to a roux limb which bypasses a large portion of the small intestine. This results in the food bypassing the majority of the stomach, the duodenum, and the first 40 to $50 \mathrm{~cm}$ of jejunum.

Mitochondrial nanotunnels: thin double-membrane protrusions that connect the matrices of non-adjacent mitochondria.

Cachexia: A complex metabolic syndrome associated with underlying illness and characterized by loss of muscle with or without loss of fat mass.

Sarcopenia: is the age-associated loss of muscle mass and strength. 


\section{CLINICIANS CORNER}

- Mitochondria are multifaceted organelles that participate in a variety of cellular functions far beyond simple energy provision.

- The ability of mitochondria to adapt to environmental stimuli and support metabolism and cellular homeostasis is governed by shifts in mitochondrial morphology, however increasing evidence suggests that aberrant mitochondrial morphology is present in numerous pathological conditions, including metabolic disease and age-associated skeletal muscle dysfunction. In addition, animal studies suggest that dysregulation of mitochondrial morphology may be mechanistically linked to pathogenesis

- Currently there are no pharmaceutical agents available to target these pathways, however, diet and exercise, and bariatric surgery in humans and animals have been effective at normalizing mitochondrial morphology and dynamics in association with improved body composition and metabolic outcomes. 
Table 2. Summary of literature linking mitochondrial morphology and dynamics in humans to age- and obesity-associated skeletal muscle dysfunction.

\begin{tabular}{|c|c|c|c|c|c|c|}
\hline Participants & Treatment/intervention & $\begin{array}{l}\text { Mitochondrial } \\
\text { Morphology }\end{array}$ & $\begin{array}{l}\text { Fusion } \\
\text { gene/protein } \\
\text { expression }\end{array}$ & $\begin{array}{c}\text { Fission } \\
\text { gene/protein } \\
\text { expression }\end{array}$ & Functional Outcomes & Refs \\
\hline \multirow{2}{*}{$\begin{array}{l}\text { Morbid obesity with } \\
\text { and without T2D vs } \\
\text { Lean Controls }\end{array}$} & & $\begin{array}{l}\uparrow \text { Fragmentation in } \\
\text { obese groups }\end{array}$ & $\begin{array}{l}\uparrow \text { Mfn1/2 in } \\
\text { Obese }\end{array}$ & $\begin{array}{l}\uparrow \text { Drp1 in Obese } \\
\text { and T2D }\end{array}$ & $\begin{array}{l}\text { - Reduced insulin sensitivity } \\
\text { in obese and T2D }\end{array}$ & \multirow[b]{2}{*}{ [33] } \\
\hline & $\begin{array}{c}\text { Roux-en-Y Gastric } \\
\text { Bypass }\end{array}$ & $\begin{array}{c}\downarrow \text { Fragmentation in } \\
\text { some obese and T2D } \\
\text { patients }\end{array}$ & & $\downarrow$ Fis1 & $\begin{array}{l}\text { - }{ }_{\text {RYGulin Sensitivity after }} \\
\text { RYG }\end{array}$ & \\
\hline Young Healthy & Lipid Infusion & $\uparrow$ Fragmentation & No Change & $\uparrow$ Drp1ser616 & $\begin{array}{l}\text { - Reduced insulin } \\
\text { sensitivity. } \\
\text { - No change in } \\
\text { mitochondrial capacity. } \\
\text { - Reduced mitochondrial } \\
\text { membrane potential. } \\
\end{array}$ & [35] \\
\hline $\begin{array}{l}\text { Cultured Myotubes } \\
\text { from severely obese } \\
\text { and T2D patients vs } \\
\text { young lean healthy }\end{array}$ & & $\begin{array}{l}\uparrow \text { Fragmentation in } \\
\text { Obesity and T2D }\end{array}$ & & $\begin{array}{c}\uparrow \text { Drp } 1 \text { ser616 in } \\
\text { Obesity and T2D }\end{array}$ & & [36] \\
\hline $\begin{array}{l}\text { Cultured Myotubes } \\
\text { from severely obese } \\
\text { and T2D patients }\end{array}$ & $\begin{array}{c}\text { Roux-en-Y Gastric } \\
\text { Bypass }\end{array}$ & $\begin{array}{l}\downarrow \text { Fragmentation } \\
\text { following surgery }\end{array}$ & & $\begin{array}{l}\downarrow \text { Drp } 1^{\text {ser616 }} \\
\text { following surgery }\end{array}$ & & [37] \\
\hline $\begin{array}{l}\text { Obese and T2D vs } \\
\text { lean controls }\end{array}$ & & & $\begin{array}{l}\downarrow \text { Mfn2 in Obese } \\
\text { and T2D }\end{array}$ & & & [30] \\
\hline $\begin{array}{l}\text { Severely Obese } \\
\text { Females }\end{array}$ & Bariatric Surgery & & $\begin{array}{c}\downarrow \text { Mfn2 mRNA } \\
\text { following weight } \\
\text { loss } \\
\end{array}$ & & $\begin{array}{l}\text { - } \downarrow \text { Mfn2 mRNA correlated } \\
\text { with improved insulin } \\
\text { sensitivity } \\
\end{array}$ & {$[40]$} \\
\hline $\begin{array}{l}\text { Elderly with Heart } \\
\text { Failure vs Healthy } \\
\text { age-matched controls }\end{array}$ & & & $\begin{array}{l}\quad \downarrow \text { Mfn2 in } \\
\text { Patients with } \\
\text { Heart Failure }\end{array}$ & & $\begin{array}{l}\text { - Mfn2 was positively } \\
\text { associated with exercise } \\
\text { performance }\end{array}$ & [41] \\
\hline
\end{tabular}




\begin{tabular}{|c|c|c|c|c|c|c|}
\hline Participants & Treatment/intervention & $\begin{array}{l}\text { Mitochondrial } \\
\text { Morphology }\end{array}$ & $\begin{array}{l}\text { Fusion } \\
\text { gene/protein } \\
\text { expression }\end{array}$ & $\begin{array}{l}\text { Fission } \\
\text { gene/protein } \\
\text { expression }\end{array}$ & - Functional Outcomes & Refs \\
\hline $\begin{array}{l}\text { Healthy } \\
\text { Premenopausal } \\
\text { Women }\end{array}$ & $\begin{array}{l}\text { Hyperinsulinemic- } \\
\text { euglycemic clamp }\end{array}$ & & No Change & No Change & $\begin{array}{c}\text { - Association between Mfn2 } \\
\text { expression and ROS }\end{array}$ & [44] \\
\hline $\begin{array}{c}\text { Lean Healthy, } \\
\text { Obese, T2D, Athletes }\end{array}$ & & $\begin{array}{l}\uparrow \text { Fragmentation in } \\
\text { T2D relative to } \\
\text { athletes }\end{array}$ & $\begin{array}{l}\text { OPA1 highest in } \\
\text { athletes and } \\
\text { lowest in T2D. }\end{array}$ & $\begin{array}{l}\text { Fis1 highest in } \\
\text { athletes and } \\
\text { lowest in T2D. }\end{array}$ & & [45] \\
\hline $\begin{array}{l}\text { Older Obese } \\
\text { individuals }\end{array}$ & 12wk diet and exercise & & $\begin{array}{l}\uparrow \text { OPA1 following } \\
\text { intervention }\end{array}$ & & & [46] \\
\hline $\begin{array}{l}\text { Morbidly obese } \\
\text { women }\end{array}$ & $\begin{array}{c}\text { Roux-en-Y Gastric } \\
\text { Bypass }\end{array}$ & & $\begin{array}{l}\downarrow \text { Mfn2 mRNA } \\
\text { following weight } \\
\text { loss surgery }\end{array}$ & & $\begin{array}{l}\text { - Association between } \\
\text { Increased MFN2 and } \\
\text { glucose uptake following } \\
\text { surgery }\end{array}$ & [48] \\
\hline $\begin{array}{l}\text { Obese vs age } \\
\text { matched lean }\end{array}$ & & & No Difference & No Difference & & [49] \\
\hline $\begin{array}{l}\text { T2D patients vs } \\
\text { weight matched } \\
\text { controls }\end{array}$ & & & No Difference & $\begin{array}{c}\text { Drp1 mRNA } \\
\text { (DNM1L) was } \\
\text { lower in the T2D } \\
\text { group }\end{array}$ & & {$[50]$} \\
\hline $\begin{array}{l}\text { Older Obese } \\
\text { individuals }\end{array}$ & 12wk exercise & & $\begin{array}{l}\text { } O P A 1 \mathrm{mRNA} \\
\text { after exercise } \\
\text { training }\end{array}$ & $\downarrow$ Drp1 $1^{\text {ser616 }}$ & $\begin{array}{l}\text { - Association between } \\
\text { changes in Drp1ser616 } \\
\text { and improvements in } \\
\text { insulin sensitivity and fat } \\
\text { oxidation }\end{array}$ & {$[56]$} \\
\hline $\begin{array}{l}\text { Young active, Young } \\
\text { sedentary, Middle- } \\
\text { aged sedentary, and } \\
\text { Older sedentary }\end{array}$ & & & $\begin{array}{c}\uparrow \text { OPA1 and } \\
\text { Mfn2 in Young } \\
\text { active }\end{array}$ & $\begin{array}{l}\uparrow \text { Fis1 and Drp1 } \\
\text { in Young active } \\
\downarrow \text { Drp1 in Middle } \\
\text { aged sedentary }\end{array}$ & $\begin{array}{l}\text { Negative relationship } \\
\text { between Fusion and } \\
\text { fission proteins with BMI }\end{array}$ & [10] \\
\hline
\end{tabular}




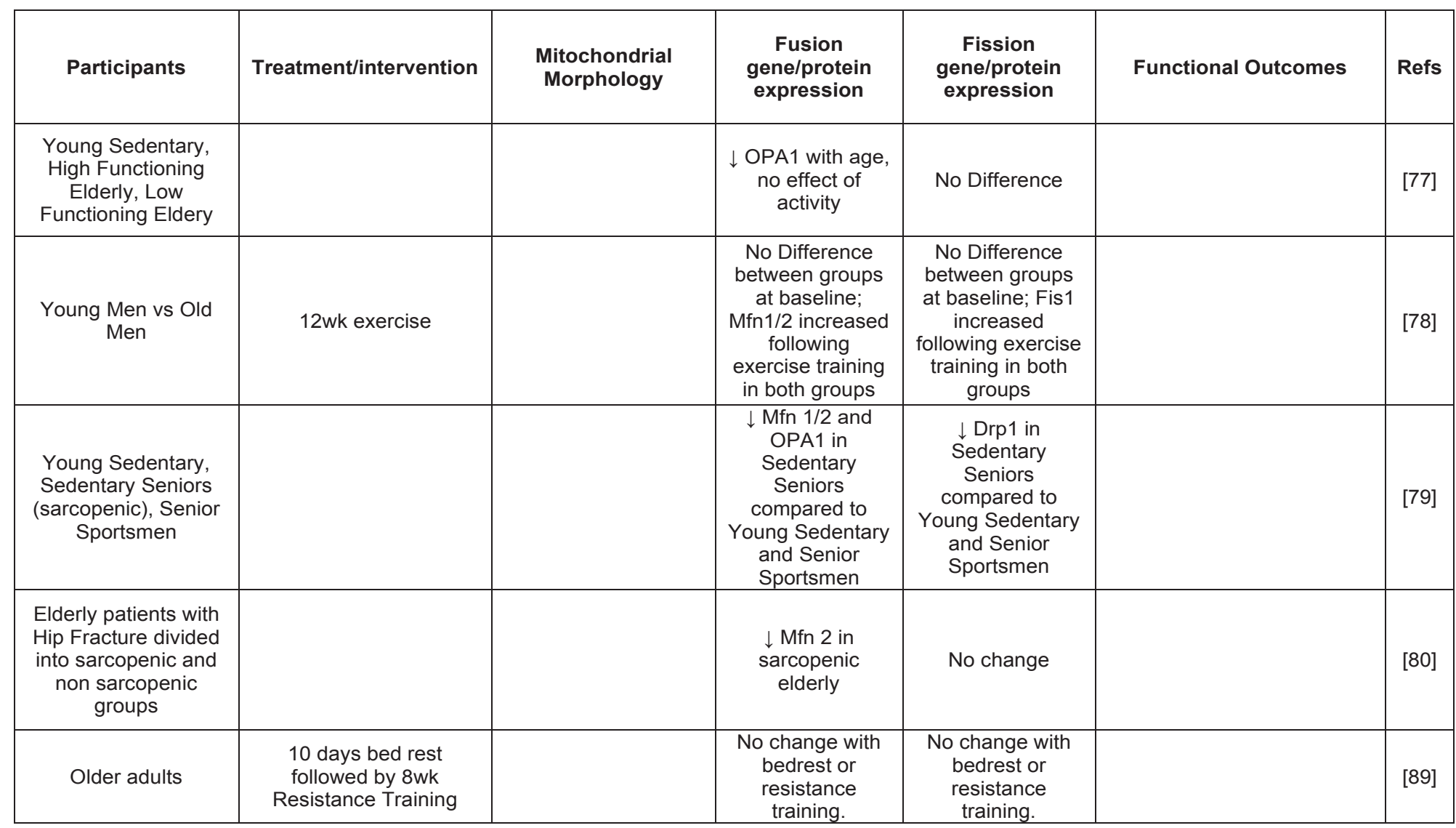




\section{REFERENCES}

1. Kaeberlein, M. (2018) How healthy is the healthspan concept? Geroscience 40 (4), 361364.

2. Chang, V.W. et al. (2017) Trends in the Relationship Between Obesity and Disability, 1988-2012. Am J Epidemiol 186 (6), 688-695.

3. Kanasi, E. et al. (2016) The aging population: demographics and the biology of aging. Periodontol 200072 (1), 13-8.

4. Mehta, T. et al. (2014) Obesity and mortality: are the risks declining? Evidence from multiple prospective studies in the United States. Obes Rev 15 (8), 619-29.

5. Batsis, J.A. et al. (2014) Sarcopenia, sarcopenic obesity and mortality in older adults: results from the National Health and Nutrition Examination Survey III. Eur J Clin Nutr 68 (9), 1001-7.

6. Baumgartner, R.N. et al. (2004) Sarcopenic obesity predicts instrumental activities of daily living disability in the elderly. Obes Res 12 (12), 1995-2004.

7. Abbasi, F. et al. (2002) Relationship between obesity, insulin resistance, and coronary heart disease risk. J Am Coll Cardiol 40 (5), 937-43.

8. Chambers, T.L. et al. (2020) Skeletal muscle size, function, and adiposity with lifelong aerobic exercise. J Appl Physiol (1985) 128 (2), 368-378.

9. St-Jean-Pelletier, F. et al. (2017) The impact of ageing, physical activity, and pre-frailty on skeletal muscle phenotype, mitochondrial content, and intramyocellular lipids in men. $J$ Cachexia Sarcopenia Muscle 8 (2), 213-228.

10. Distefano, G. et al. (2017) Chronological Age Does not Influence Ex-vivo Mitochondrial Respiration and Quality Control in Skeletal Muscle. J Gerontol A Biol Sci Med Sci 72 (4), 535-542.

11. Kang, Y.M. et al. (2017) Visceral adiposity index predicts the conversion of metabolically healthy obesity to an unhealthy phenotype. PLoS One 12 (6), e0179635.

12. Okamura, T. et al. (2019) Ectopic fat obesity presents the greatest risk for incident type 2 diabetes: a population-based longitudinal study. Int J Obes (Lond) 43 (1), 139-148.

13. Murton, A.J. et al. (2015) Obesity Appears to Be Associated With Altered Muscle Protein Synthetic and Breakdown Responses to Increased Nutrient Delivery in Older Men, but Not Reduced Muscle Mass or Contractile Function. Diabetes 64 (9), 3160-71.

14. Yagi, S. et al. (2014) Association of lower limb muscle mass and energy expenditure with visceral fat mass in healthy men. Diabetol Metab Syndr 6 (1), 27.

15. Gupte, T.M. (2015) Mitochondrial Fragmentation Due to Inhibition of Fusion Increases Cyclin B through Mitochondrial Superoxide Radicals. PLoS One 10 (5), e0126829.

16. Vincent, A.E. et al. (2019) Quantitative 3D Mapping of the Human Skeletal Muscle Mitochondrial Network. Cell Rep 26 (4), 996-1009 e4.

17. Glancy, B. et al. (2015) Mitochondrial reticulum for cellular energy distribution in muscle. Nature 523 (7562), 617-20.

18. Glancy, B. et al. (2017) Power Grid Protection of the Muscle Mitochondrial Reticulum. Cell Rep 19 (3), 487-496. 
19. Nunnari, J. et al. (1997) Mitochondrial transmission during mating in Saccharomyces cerevisiae is determined by mitochondrial fusion and fission and the intramitochondrial segregation of mitochondrial DNA. Mol Biol Cell 8 (7), 1233-42.

20. Al-Mehdi, A.B. et al. (2012) Perinuclear mitochondrial clustering creates an oxidant-rich nuclear domain required for hypoxia-induced transcription. Sci Signal 5 (231), ra47.

21. Lalia, A.Z. et al. (2016) Predictors of Whole-Body Insulin Sensitivity Across Ages and Adiposity in Adult Humans. J Clin Endocrinol Metab 101 (2), 626-34.

22. Toyama, E.Q. et al. (2016) Metabolism. AMP-activated protein kinase mediates mitochondrial fission in response to energy stress. Science 351 (6270), 275-281.

23. Wu, W. et al. (2016) FUNDC1 regulates mitochondrial dynamics at the ER-mitochondrial contact site under hypoxic conditions. EMBO J 35 (13), 1368-84.

24. Favaro, G. et al. (2019) DRP1-mediated mitochondrial shape controls calcium homeostasis and muscle mass. Nat Commun 10 (1), 2576.

25. Hung, C.H. et al. (2018) A reciprocal relationship between reactive oxygen species and mitochondrial dynamics in neurodegeneration. Redox Biol 14, 7-19.

26. Baker, N. et al. (2019) Linking mitochondrial dynamics, cristae remodeling and supercomplex formation: How mitochondrial structure can regulate bioenergetics. Mitochondrion 49, 259-268.

27. Liu, Y.J. et al. (2020) Mitochondrial fission and fusion: A dynamic role in aging and potential target for age-related disease. Mech Ageing Dev 186, 111212.

28. Zhang, Q. et al. (2019) ROCK1 induces dopaminergic nerve cell apoptosis via the activation of Drp1-mediated aberrant mitochondrial fission in Parkinson's disease. Exp Mol Med 51 (10), 1-13.

29. Manczak, M. et al. (2011) Impaired mitochondrial dynamics and abnormal interaction of amyloid beta with mitochondrial protein Drp1 in neurons from patients with Alzheimer's disease: implications for neuronal damage. Hum Mol Genet 20 (13), 2495-509.

30. Bach, D. et al. (2003) Mitofusin-2 determines mitochondrial network architecture and mitochondrial metabolism. A novel regulatory mechanism altered in obesity. J Biol Chem 278 (19), $17190-7$.

31. Jheng, H.F. et al. (2012) Mitochondrial fission contributes to mitochondrial dysfunction and insulin resistance in skeletal muscle. Mol Cell Biol 32 (2), 309-19.

32. Liu, R. et al. (2014) Impaired mitochondrial dynamics and bioenergetics in diabetic skeletal muscle. PLoS One 9 (3), e92810.

33. Kristensen, M.D. et al. (2018) Obesity leads to impairments in the morphology and organization of human skeletal muscle lipid droplets and mitochondrial networks, which are resolved with gastric bypass surgery-induced improvements in insulin sensitivity. Acta Physiol (Oxf) 224 (4), e13100.

34. Vijgen, G.H. et al. (2013) Impaired skeletal muscle mitochondrial function in morbidly obese patients is normalized one year after bariatric surgery. Surg Obes Relat Dis 9 (6), 936-41. 
35. Axelrod, C.L. et al. (2021) Lipids activate skeletal muscle mitochondrial fission and quality control networks to induce insulin resistance in humans. Metabolism 121, 154803.

36. Gundersen, A.E. et al. (2020) Altered mitochondrial network morphology and regulatory proteins in mitochondrial quality control in myotubes from severely obese humans with or without type 2 diabetes. Appl Physiol Nutr Metab 45 (3), 283-293.

37. Kugler, B.A. et al. (2020) Roux-en-Y gastric bypass surgery restores insulin-mediated glucose partitioning and mitochondrial dynamics in primary myotubes from severely obese humans. Int J Obes (Lond) 44 (3), 684-696.

38. Pich, S. et al. (2005) The Charcot-Marie-Tooth type 2A gene product, Mfn2, up-regulates fuel oxidation through expression of OXPHOS system. Hum Mol Genet 14 (11), 1405-15.

39. Greene, N.P. et al. (2015) Mitochondrial quality control, promoted by PGC-1alpha, is dysregulated by Western diet-induced obesity and partially restored by moderate physical activity in mice. Physiol Rep 3 (7).

40. Mingrone, G. et al. (2005) Could the low level of expression of the gene encoding skeletal muscle mitofusin-2 account for the metabolic inflexibility of obesity? Diabetologia 48 (10), 2108-14.

41. Molina, A.J. et al. (2016) Skeletal Muscle Mitochondrial Content, Oxidative Capacity, and Mfn2 Expression Are Reduced in Older Patients With Heart Failure and Preserved Ejection Fraction and Are Related to Exercise Intolerance. JACC Heart Fail 4 (8), 636-45.

42. Nie, Q. et al. (2014) Mitofusin 2 deficiency leads to oxidative stress that contributes to insulin resistance in rat skeletal muscle cells. Mol Biol Rep 41 (10), 6975-83.

43. Kong, D. et al. (2013) Overexpression of mitofusin 2 improves translocation of glucose transporter 4 in skeletal muscle of highfat dietfed rats through AMPactivated protein kinase signaling. Mol Med Rep 8 (1), 205-10.

44. Warren, J.L. et al. (2017) Effects of acute hyperinsulinemia on skeletal muscle mitochondrial function, reactive oxygen species production, and metabolism in premenopausal women. Metabolism 77, 1-12.

45. Houzelle, A. et al. (2021) Human skeletal muscle mitochondrial dynamics in relation to oxidative capacity and insulin sensitivity. Diabetologia 64 (2), 424-436.

46. Axelrod, C.L. et al. (2019) Exercise training remodels human skeletal muscle mitochondrial fission and fusion machinery towards a pro-elongation phenotype. Acta Physiol (Oxf) 225 (4), e13216.

47. Sacks, J. et al. (2018) Effect of Roux-en-Y gastric bypass on liver mitochondrial dynamics in a rat model of obesity. Physiol Rep 6 (4).

48. Gastaldi, G. et al. (2007) Upregulation of peroxisome proliferator-activated receptor gamma coactivator gene (PGC1A) during weight loss is related to insulin sensitivity but not to energy expenditure. Diabetologia 50 (11), 2348-55.

49. Holloway, G.P. et al. (2008) PGC-1alpha's relationship with skeletal muscle palmitate oxidation is not present with obesity despite maintained PGC-1alpha and PGC-1beta protein. Am J Physiol Endocrinol Metab 294 (6), E1060-9. 
50. Kruse, R. et al. (2017) Intact initiation of autophagy and mitochondrial fission by acute exercise in skeletal muscle of patients with Type 2 diabetes. Clin Sci (Lond) 131 (1), 37-47.

51. Boutant, M. et al. (2017) Mfn2 is critical for brown adipose tissue thermogenic function. EMBO J 36 (11), 1543-1558.

52. Sebastian, D. et al. (2012) Mitofusin 2 (Mfn2) links mitochondrial and endoplasmic reticulum function with insulin signaling and is essential for normal glucose homeostasis. Proc Natl Acad Sci U S A 109 (14), 5523-8.

53. Rieusset, J. (2018) The role of endoplasmic reticulum-mitochondria contact sites in the control of glucose homeostasis: an update. Cell Death Dis 9 (3), 388.

54. Arruda, A.P. et al. (2014) Chronic enrichment of hepatic endoplasmic reticulummitochondria contact leads to mitochondrial dysfunction in obesity. Nat Med 20 (12), 142735 .

55. del Campo, A. et al. (2014) Mitochondrial fragmentation impairs insulin-dependent glucose uptake by modulating Akt activity through mitochondrial Ca2+ uptake. Am J Physiol Endocrinol Metab 306 (1), E1-E13.

56. Fealy, C.E. et al. (2014) Exercise training decreases activation of the mitochondrial fission protein dynamin-related protein-1 in insulin-resistant human skeletal muscle. J Appl Physiol (1985) 117 (3), 239-45.

57. Smith, M.E. et al. (2013) Mitochondrial fission mediates ceramide-induced metabolic disruption in skeletal muscle. Biochem J 456 (3), 427-39.

58. Hammerschmidt, P. et al. (2019) CerS6-Derived Sphingolipids Interact with Mff and Promote Mitochondrial Fragmentation in Obesity. Cell 177 (6), 1536-1552 e23.

59. Reali, F. et al. (2017) Mechanistic interplay between ceramide and insulin resistance. Sci Rep 7, 41231.

60. Castro-Sepulveda, M. et al. (2020) Relative lipid oxidation associates directly with mitochondrial fusion phenotype and mitochondria-sarcoplasmic reticulum interactions in human skeletal muscle. Am J Physiol Endocrinol Metab 318 (6), E848-E855.

61. Luft, R. et al. (1962) A case of severe hypermetabolism of nonthyroid origin with a defect in the maintenance of mitochondrial respiratory control: a correlated clinical, biochemical, and morphological study. J Clin Invest 41, 1776-804.

62. Lombes, A. et al. (1989) Mitochondrial encephalomyopathies. Rev Neurol (Paris) 145 (10), 671-89.

63. Zuchner, S. et al. (2004) Mutations in the mitochondrial GTPase mitofusin 2 cause Charcot-Marie-Tooth neuropathy type 2A. Nat Genet 36 (5), 449-51.

64. Delettre, C. et al. (2000) Nuclear gene OPA1, encoding a mitochondrial dynamin-related protein, is mutated in dominant optic atrophy. Nat Genet 26 (2), 207-10.

65. Bartsakoulia, M. et al. (2018) A novel mechanism causing imbalance of mitochondrial fusion and fission in human myopathies. Hum Mol Genet 27 (7), 1186-1195.

66. Barreto, R. et al. (2016) Cancer and Chemotherapy Contribute to Muscle Loss by Activating Common Signaling Pathways. Front Physiol 7, 472. 
67. van der Ende, M. et al. (2018) Mitochondrial dynamics in cancer-induced cachexia. Biochim Biophys Acta Rev Cancer 1870 (2), 137-150.

68. Mitchell, W.K. et al. (2012) Sarcopenia, dynapenia, and the impact of advancing age on human skeletal muscle size and strength; a quantitative review. Front Physiol 3, 260.

69. Gouspillou, G. et al. (2014) Mitochondrial energetics is impaired in vivo in aged skeletal muscle. Aging Cell 13 (1), 39-48.

70. Chabi, B. et al. (2008) Mitochondrial function and apoptotic susceptibility in aging skeletal muscle. Aging Cell 7 (1), 2-12.

71. Navratil, M. et al. (2008) Giant mitochondria do not fuse and exchange their contents with normal mitochondria. Exp Cell Res 314 (1), 164-72.

72. Pietrangelo, L. et al. (2015) Age-dependent uncoupling of mitochondria from Ca2(+) release units in skeletal muscle. Oncotarget 6 (34), 35358-71.

73. Huang, D.D. et al. (2019) Nrf2 deficiency exacerbates frailty and sarcopenia by impairing skeletal muscle mitochondrial biogenesis and dynamics in an age-dependent manner. Exp Gerontol 119, 61-73.

74. Pereira, R.O. et al. (2017) OPA1 deficiency promotes secretion of FGF21 from muscle that prevents obesity and insulin resistance. EMBO J 36 (14), 2126-2145.

75. Del Campo, A. et al. (2018) Muscle function decline and mitochondria changes in middle age precede sarcopenia in mice. Aging (Albany NY) 10 (1), 34-55.

76. Bori, Z. et al. (2012) The effects of aging, physical training, and a single bout of exercise on mitochondrial protein expression in human skeletal muscle. Exp Gerontol 47 (6), 417-24.

77. Joseph, A.M. et al. (2013) Dysregulation of mitochondrial quality control processes contribute to sarcopenia in a mouse model of premature aging. PLoS One 8 (7), e69327.

78. Konopka, A.R. et al. (2014) Markers of human skeletal muscle mitochondrial biogenesis and quality control: effects of age and aerobic exercise training. J Gerontol A Biol Sci Med Sci 69 (4), 371-8.

79. Tezze, C. et al. (2017) Age-Associated Loss of OPA1 in Muscle Impacts Muscle Mass, Metabolic Homeostasis, Systemic Inflammation, and Epithelial Senescence. Cell Metab 25 (6), 1374-1389 e6.

80. Marzetti, E. et al. (2016) Association between myocyte quality control signaling and sarcopenia in old hip-fractured patients: Results from the Sarcopenia in HIp FracTure (SHIFT) exploratory study. Exp Gerontol 80, 1-5.

81. Sebastian, D. et al. (2016) Mfn2 deficiency links age-related sarcopenia and impaired autophagy to activation of an adaptive mitophagy pathway. EMBO J 35 (15), 1677-93.

82. Masiero, E. and Sandri, M. (2010) Autophagy inhibition induces atrophy and myopathy in adult skeletal muscles. Autophagy 6 (2), 307-9.

83. Carnio, S. et al. (2014) Autophagy impairment in muscle induces neuromuscular junction degeneration and precocious aging. Cell Rep 8 (5), 1509-21.

84. Kroemer, G. (2015) Autophagy: a druggable process that is deregulated in aging and human disease. J Clin Invest 125 (1), 1-4. 
85. Ding, Y. et al. (2015) Mitofusin 2-deficiency suppresses cell proliferation through disturbance of autophagy. PLoS One 10 (3), e0121328.

86. Kang, C. and Ji, L.L. (2016) PGC-1alpha overexpression via local transfection attenuates mitophagy pathway in muscle disuse atrophy. Free Radic Biol Med 93, 32-40.

87. Rodriguez-Nuevo, A. et al. (2018) Mitochondrial DNA and TLR9 drive muscle inflammation upon Opa1 deficiency. EMBO J 37 (10).

88. Lee, Y.J. et al. (2020) Down-regulation of the mitochondrial i-AAA protease Yme1L induces muscle atrophy via FoxO3a and myostatin activation. J Cell Mol Med 24 (1), 899 909.

89. Standley, R.A. et al. (2017) Effects of beta-hydroxy-beta-methylbutyrate on skeletal muscle mitochondrial content and dynamics, and lipids after 10 days of bed rest in older adults. J Appl Physiol (1985) 123 (5), 1092-1100.

90. Romanello, V. et al. (2019) Inhibition of the Fission Machinery Mitigates OPA1 Impairment in Adult Skeletal Muscles. Cells 8 (6).

91. Song, M. et al. (2017) Abrogating Mitochondrial Dynamics in Mouse Hearts Accelerates Mitochondrial Senescence. Cell Metab 26 (6), 872-883 e5.

92. Lee, H. et al. (2017) Oleate Prevents Palmitate-Induced Atrophy via Modulation of Mitochondrial ROS Production in Skeletal Myotubes. Oxid Med Cell Longev 2017, 2739721.

93. Santel, A. et al. (2003) Mitofusin-1 protein is a generally expressed mediator of mitochondrial fusion in mammalian cells. J Cell Sci 116 (Pt 13), 2763-74.

94. de Brito, O.M. and Scorrano, L. (2008) Mitofusin 2: a mitochondria-shaping protein with signaling roles beyond fusion. Antioxid Redox Signal 10 (3), 621-33.

95. Naon, D. et al. (2016) Critical reappraisal confirms that Mitofusin 2 is an endoplasmic reticulum-mitochondria tether. Proc Natl Acad Sci U S A 113 (40), 11249-11254.

96. Ainbinder, A. et al. (2015) Role of Mitofusin-2 in mitochondrial localization and calcium uptake in skeletal muscle. Cell Calcium 57 (1), 14-24.

97. Chen, Y. and Dorn, G.W., 2nd (2013) PINK1-phosphorylated mitofusin 2 is a Parkin receptor for culling damaged mitochondria. Science 340 (6131), 471-5.

98. Del Dotto, V. et al. (2017) OPA1 Isoforms in the Hierarchical Organization of Mitochondrial Functions. Cell Rep 19 (12), 2557-2571.

99. Hu, C. et al. (2017) Drp1-Dependent Mitochondrial Fission Plays Critical Roles in Physiological and Pathological Progresses in Mammals. Int J Mol Sci 18 (1). 


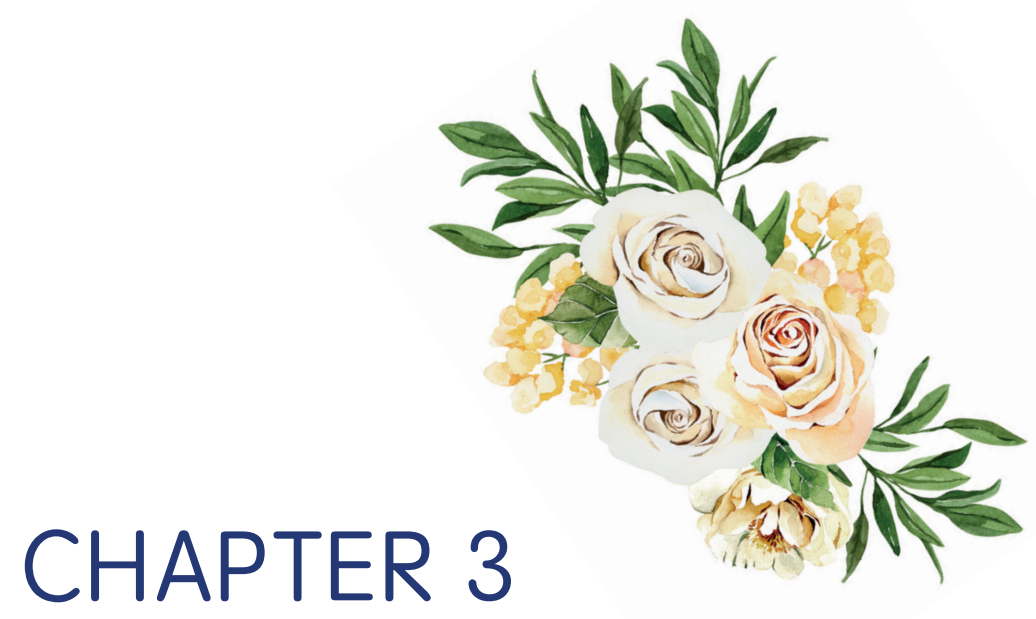

\section{Impact of aging and exercise on skeletal muscle mitochondrial capacity, energy metabolism, and physical function}

Lotte Grevendonk, Niels J. Connell, Christopher McCrum, Ciarán E. Fealy, Lena Bilet, Yvonne M. H. Bruls, Julian Mevenkamp, Vera B. Schrauwen-Hinderling, Johanna A. Jörgensen, Esther Moonen-Kornips, Gert Schaart, Bas Havekes, Johan de Vogel-van den Bosch, Marjolijn C.E. Bragt, Kenneth Meijer, Patrick Schrauwen, Joris Hoeks

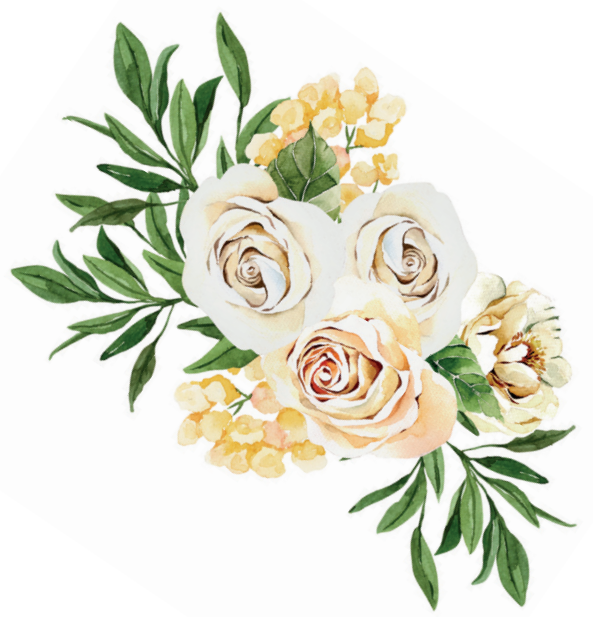




\section{Chapter 3}

\section{Abbreviations}

6MWT 6-minute walk test

${ }^{31} \mathrm{P}-\mathrm{MRS} \quad{ }^{31} \mathrm{Phosphorus} \mathrm{Magnetic} \mathrm{Resonance} \mathrm{Spectroscopy}$

ANOVA Analysis of variance

EEE Exercise energy expenditure

GEE Gross exercise efficiency

GIR Glucose infusion rate

HPA High-intensity physical activity

IO Physically impaired older adults

LPA Low-intensity physical activity

MoS Margin of Stability

MRI Magnetic Resonance Imaging

NEE Net exercise efficiency

O Older adults

OXPHOS Oxidative Phosphorylation

PA Physical activity

$\mathrm{PCr} \quad$ Phosphocreatine

Pert Perturbation

PGC-1a Peroxisome proliferator-activated receptor gamma co-activator $1 \alpha$

REE Resting energy expenditure

RER Respiratory exchange ratio

SPPB Short physical performance battery

TO Trained older adults

Y Young adults 


\section{ABSTRACT}

The relationship between the age-associated decline in mitochondrial function and its effect on skeletal muscle physiology and function remain unclear. In the current study, we examined to what extent physical activity contributes to the decline in mitochondrial function and muscle health during aging and compared mitochondrial function in young and older adults, with similar habitual physical activity levels. We also studied exercise-trained older adults and physically impaired older adults. Aging was associated with a decline in mitochondrial capacity, exercise capacity and efficiency, gait stability, muscle function, and insulin sensitivity, even when maintaining an adequate daily physical activity level. Our data also suggest that a further increase in physical activity level, achieved through regular exercise training, can largely negate the effects of aging. Finally, mitochondrial capacity correlated with exercise efficiency and insulin sensitivity. Together, our data support a link between mitochondrial function and age-associated deterioration of skeletal muscle. 


\section{INTRODUCTION}

A large portion of the world's population is of middle to older age, and in high-income countries, the proportion of people over the age of 60 is growing faster than any other age group (1). Since aging is associated with a variety of comorbidities resulting in rapidly increasing health care costs, this demographic change is one of the major societal challenges of the current decade (2).

One of the distinctive features of aging is the progressive loss of muscle mass and physical function, collectively known as sarcopenia (3). The loss of skeletal muscle mass and tissue function has been related to mobility impairments (4), such as difficulties walking short distances or standing up from a chair $(5,6)$, an increased risk of falls $(7,8)$, physical frailty $(9)$, and metabolic impairments (10), ultimately leading to a loss of physical independence and increased care need (11). Improving balance and mobility are important factors for public health in older adults in order to reduce fall-related consequences such as fractures, further functional decline, immobility, and death (12).

In parallel with the progressive loss of muscle function, mitochondrial respiratory activity in human skeletal muscle has been shown to decrease with advancing age in healthy men and women $(13,14)$. Furthermore, protein levels of the mitochondrial master regulator peroxisome proliferator-activated receptor gamma co-activator $1 \alpha$ (PGC-1a) were found to correlate with walking speed in healthy older adults (15). Some preclinical studies indeed suggest that the reduction in muscle mitochondrial function may underlie the decline in muscle health during aging. Thus, an accelerated manifestation of sarcopenia is seen in transgenic mice lacking the antioxidant enzyme superoxide dismutase 1 (SOD1), which are characterized by both a diminished mitochondrial bioenergetic function and an induction of mitochondrial-mediated apoptosis (16). In addition, in rats, age-related increases in mitochondrial abnormalities have been associated with muscle fiber splitting and atrophy (17). Therefore, it is tempting to speculate that augmenting mitochondrial function could be a potential strategy to counteract aging-associated decline in physical function.

Although some human studies have addressed age-related alterations in muscle mitochondrial function in relation to the decline in skeletal muscle function $(5,18-21)$, the available data in humans is scarce and the few available studies often focus on either the decline in muscle function or concentrate primarily on the mitochondrial 
alterations. Additionally, the age-associated decline in mitochondrial function is not completely attributable to aging per se and may also be explained, in part, by an age-related decline in physical activity (PA) (19,20). Decreased PA can adversely affect mitochondrial capacity (24), while exercise training stimulates mitochondrial biogenesis through increases in the master regulator PGC-1a (25). Older adults are more inactive with advancing age which in turn decreases functional fitness (26). Therefore, it remains unclear to what extent chronological age and physical inactivity contribute to the decline in mitochondrial function and muscle health during aging.

To delineate these relationships, we conducted a cross-sectional study with detailed phenotyping in groups of young versus older human participants, with a range in oxidative capacity and physical function. The first aim of the study was to assess if mitochondrial function is reduced in older compared to young participants with a similar level of habitual PA, and to examine how mitochondrial function relates to muscle function. To this end, we performed a wide array of assessments of skeletal muscle function (including strength, volume, insulin sensitivity, gait stability and adaptability, exercise capacity, and exercise efficiency) as well as in and ex vivo measurements to characterize mitochondrial capacity. The second aim of the study was to investigate the potential of regular exercise training in older adults for the maintenance of mitochondrial function, and if this is associated with maintained muscle health. Using the methods described above, the older adults with normal PA levels were also compared to older adults with a high PA level (trained individuals) and older adults with low physical function (physically impaired individuals, low PA). We also performed correlation analyses between skeletal muscle mitochondrial function, physical function, and muscle health during aging.

\section{RESULTS}

\section{Mitochondrial function and muscle health decrease with age}

To assess the effect of age on mitochondrial function and muscle health, we compared young $(\mathrm{Y})$ versus older $(\mathrm{O})$ individuals with comparable levels of PA. Table 1 summarizes the main participant characteristics. Average age was 24 (3) years for the young individuals $(Y)$ and 71 (4) years for the older participants ( $p<$ 0.001 ). Sex distribution was identical between the two groups (8 male and 9 female 
participants in both groups, $\mathrm{p}>0.999)$. Older individuals had an increased BMI $(\mathrm{p}=$ $0.007)$ and an increased body fat percentage $(p=0.007)$ as compared to young individuals. Fat free mass (FFM) was similar in young adults and old individuals and $(p=0.471)$. Both age groups performed a comparable amount of steps per day $(p=$ 0.146 ) and showed no significant difference in time spent on high PA level or low PA level ( $p=0.496$ and $p=0.737$, respectively) indicating comparable levels of PA in daily life between young and older participants. Furthermore, no significant differences were observed in the balance test, the 4-meter walk test, and chair-stand test, assessed during screening as part of the short physical performance battery (SPPB, $p>0.05)$.

Table 1 Participant body composition, physical activity, and physical function

\begin{tabular}{|c|c|c|}
\hline & $\begin{array}{l}\text { Young, normal } \\
\text { physical activity }(Y)\end{array}$ & $\begin{array}{l}\text { Older adults, normal } \\
\text { physical activity (O) }\end{array}$ \\
\hline $\mathbf{N}$ & 17 & 17 \\
\hline Gender $\mathbf{F} / \mathbf{M}^{\dagger}$ & $8 / 9$ & $8 / 9$ \\
\hline Age (years) & $24(3)^{*}$ & $71(4)$ \\
\hline BMI $\left(\mathbf{k g ~ m}^{-2}\right)$ & $22.7(2.9)^{*}$ & $25.8(3.3)$ \\
\hline Body weight (kg) & $69.0(10.2)$ & $74.0(11.9)$ \\
\hline Fat mass $(\%)$ & $24.4(8.2)^{*}$ & $33.1(9.2)$ \\
\hline Fat mass $(\mathrm{kg})$ & $16.8(6.1)^{*}$ & $24.5(7.9)$ \\
\hline Fat free mass $(\mathrm{kg})$ & $52.2(9.9)$ & $49.5(10.8)$ \\
\hline Steps/day & $9,983(2,781)$ & $10,207(2,832)$ \\
\hline HPA time ( $\%$ of wake time) & $2.2(1.2)$ & $2.2(1.2)$ \\
\hline LPA time ( $\%$ of wake time) & $11.4(2.4)$ & $11.7(2.6)$ \\
\hline SPPB $4 \mathrm{~m}$ walk speed $\left(\mathrm{m} \mathrm{s}^{-1}\right)$ & $1.1(0.2)$ & $1.1(0.2)$ \\
\hline SPPB Chair-stand test (s) & $9.1(2.6)$ & $10.1(1.4)$ \\
\hline \multicolumn{3}{|c|}{$\begin{array}{l}\text { Data are presented as mean (SD) * indicates significant difference between } 2 \text { groups }(p \\
0.05 \text { two-sided, independent samples t-test). }{ }^{+} \text {Sex distribution across groups was tested by } \\
\mathrm{X}^{2} \text { test }(\mathrm{p}>0.999) \text {. Body composition and physical activity could not be measured in on } \\
\text { participant of the } \mathrm{Y} \text { group due to the implications of the SARS-CoV- } 19 \text { crisis. BMI = bod } \\
\text { mass index; HPA = high-intensity physical activity; LPA }=\text { low-intensity physical activity } \\
\text { SPPB = short physical performance battery. }\end{array}$} \\
\hline
\end{tabular}

\section{Lower muscle strength, volume, and endurance in older adults}

During the 6-minute walk test (6MWT), older adults covered $\sim 9 \%$ less distance, in comparison with the young participants $(p=0.032$, Figure $1 \mathrm{a})$. Older adults also had a lower cardiorespiratory fitness, as indicated by a $\sim 26 \%$ lower maximal aerobic capacity $\left(\mathrm{VO}_{2} \mathrm{max}\right.$ ), in comparison with the young individuals ( $\mathrm{p}<0.001$, Figure $1 \mathrm{~b}$ ). Likewise, maximal power output was lower in older individuals as compared to young 
( $p<0.001$, Figure 1c). In addition, a lower isokinetic muscle strength was observed in older adults as compared to young ( $<<0.001$, Figure $1 d)$. In line with the similar FFM, muscle volume, measured in the upper leg by MRI, was comparable between young and older individuals ( $p=0.151$, Figure $1 \mathrm{e})$.

\section{Reduced exercise efficiency in older adults}

Besides maximal exercise capacity, we also assessed exercise efficiency and substrate utilization during a submaximal exercise test. Resting energy expenditure was found to be comparable between young and old ( $p=0.275$, Figure 1f) and no differences were observed in respiratory exchange rate (RER) between old and young, both when measured during the submaximal exercise ( $p=0.624$ Figure $1 \mathrm{~g}$ ) and resting conditions $(p=0.852$, Figure $1 \mathrm{~g})$. Both gross $(p<0.001)$ and net $(p<$ 0.001 ) exercise efficiency were found to be lower in the older individuals as compared to the young participants (Figure 1h). 

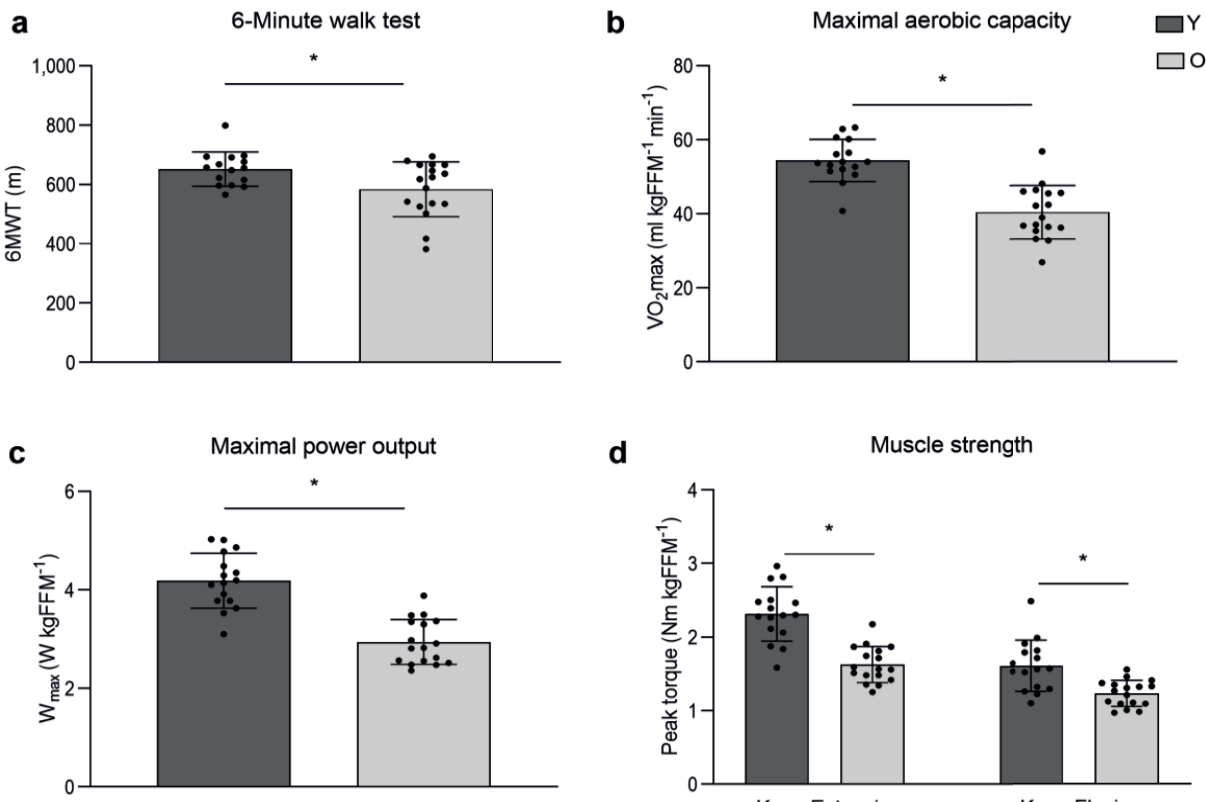

d

Muscle strength
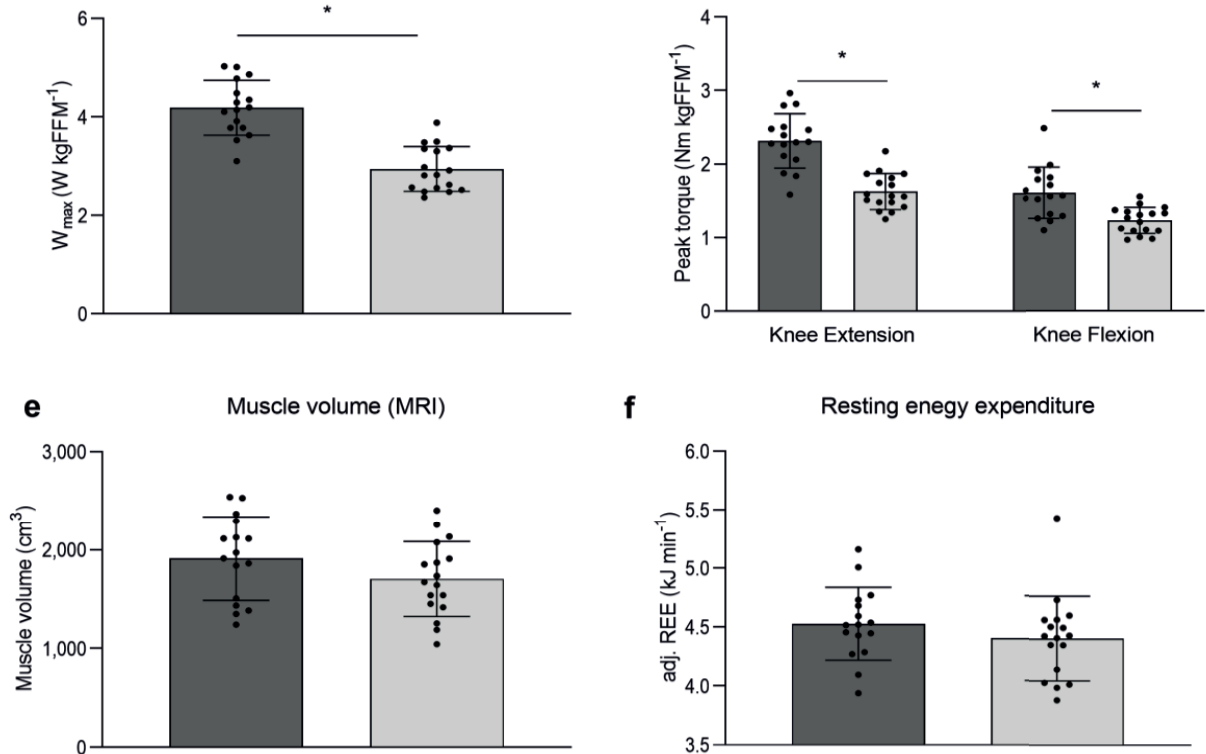

f Resting enegy expenditure
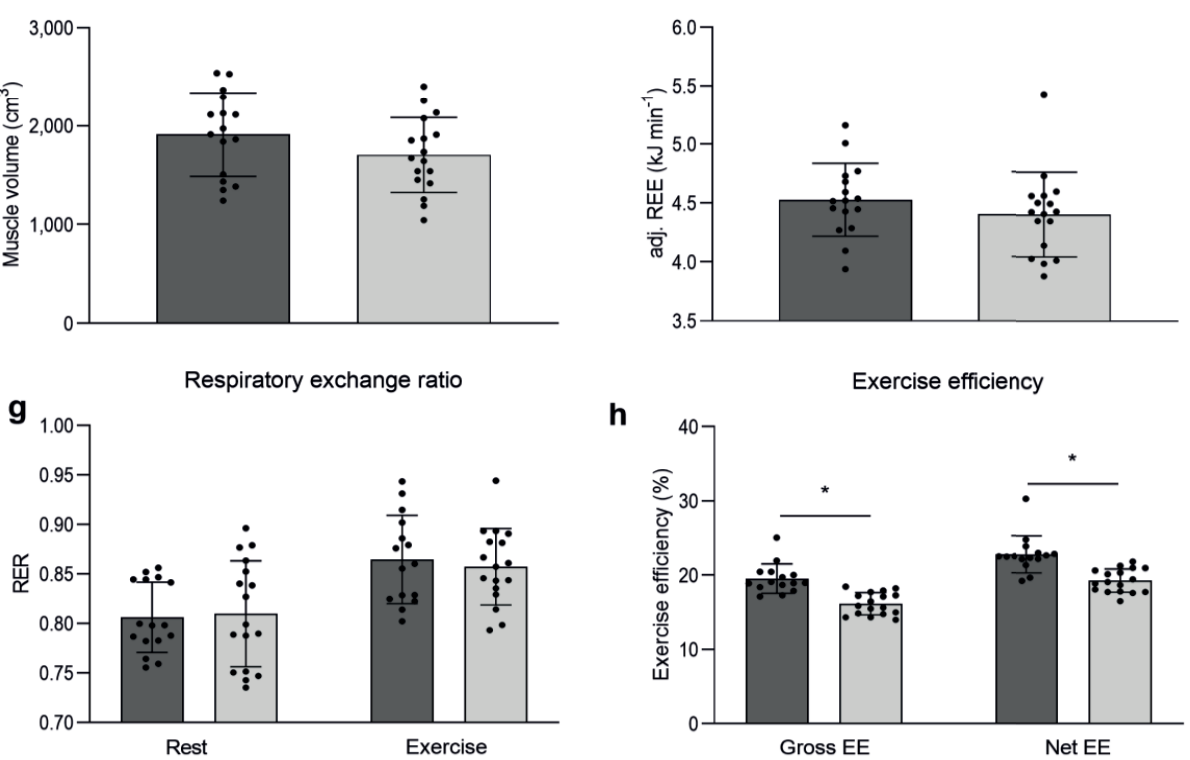

Figure 1 Effect of aging on muscle health and exercise efficiency. 
Figure 1 Effect of aging on muscle health and exercise efficiency. a Walking distance during the 6MWT performed on the Caren-system $(\mathrm{Y}, \mathrm{n}=14 ; \mathrm{O}, \mathrm{n}=17)$. b Maximum rate of oxygen consumption measured during graded cycling exercise corrected for FFM $(\mathrm{Y}, \mathrm{n}=16 ; \mathrm{O}, \mathrm{n}=17)$. c Maximal power output measured during graded cycling test $(\mathrm{Y}, \mathrm{n}$ $=16 ; \mathrm{E}, \mathrm{n}=17$ ). $\mathbf{d}$ Muscle strength expressed as the extension and flexion peak torque during an isokinetic protocol on the Biodex system and corrected for fat-free mass $(\mathrm{Y}, \mathrm{n}=$ 16; $\mathrm{O}, \mathrm{n}=17)$. e Upper leg muscle volume measured by $\mathrm{MRI}(\mathrm{Y}, \mathrm{n}=17 ; \mathrm{O}, \mathrm{n}=17)$. $\mathbf{f}$ Resting energy expenditure adjusted for fat-free mass ( $Y, n=15 ; O, n=17)$. g Respiratory exchange rate measured before, in resting conditions, and during submaximal exercise ( $\mathrm{Y}$, $\mathrm{n}=15 ; \mathrm{O}, \mathrm{n}=17)$. $\mathbf{h}$ Exercise efficiency measured during the submaximal cycle test and expressed as gross efficiency and net efficiency $(Y, n=15 ; O, n=17)$. Dark grey bars represent the young individuals $(\mathrm{Y})$, light grey bars represent the older individuals $(\mathrm{O})$. FFM could not be measured in one $Y$ due to the implications of the SARS-CoV-19 outbreak. For the same reason, 6MWT data and muscle volume data are missing in the same $Y$ participant. Another participant from $Y$ did not perform the 6MWT due to scheduling issues. The reported 6MWT distance from one $\mathrm{Y}$ participant was invalid and therefore excluded for analysis. One $Y$ participant failed to complete the submaximal cycle test due to exhaustion. In another $Y$ participant, the test could not be performed due to the implications of the SARS-CoV-19 outbreak. Values are presented as mean \pm SD (with individual data points), * denotes significant differences between the two groups ( $p<0.05$, two-sided, independent samples t-test). 6MWT = 6-minute walk test; $\mathrm{VO}_{2}$ max $=$ maximal oxygen flow; $\mathrm{Nm}=$ newton meters; $\mathrm{FFM}=$ fat-free mass; $\mathrm{RER}=$ respiratory exchange rate; $\mathrm{REE}=$ resting energy expenditure; $\mathrm{EE}=$ exercise efficiency.

\section{Lower whole-body insulin sensitivity in older adults}

To further characterize skeletal muscle health, insulin sensitivity was determined during a hyperinsulinemic-euglycemic clamp. During the clamp, the M-value required to maintain euglycemia, was $\sim 22 \%$ lower in older in comparison with young individuals ( $p=0.050$, Figure $2 a$ ), suggesting a lower whole body insulin sensitivity in older adults. Plasma insulin levels during the clamp were different between young and older adults (Y: 75.61 (9.83) $\mathrm{mU} \mathrm{l}^{-1}$ vs. O: 88.29 (10.73) $\mathrm{mU} \mathrm{l}^{-1}$ ), $\mathrm{p}=0.001$ ). Peripheral insulin sensitivity, defined as the insulin-stimulated glucose uptake (Rd) corrected for differences in insulin and glucose levels $\left(\mathrm{S}_{\mathrm{i}}\right)$, was also significantly lower in older vs. young individuals when expressed per $\mathrm{kg}$ body weight (O: 0.050 (0.028) $\mu \mathrm{mol} \mathrm{kgBW}{ }^{-1} \mathrm{~min}^{-1}$ vs. Y: 0.073 (0.024) $\left.\mu \mathrm{mol} \mathrm{kgBW}{ }^{-1} \mathrm{~min}^{-1}, \mathrm{p}=0.014\right)$. This difference was largely retained when $S_{i}$ was corrected for FFM, although it no longer reached statistical significance ( $p=0.069$, Figure $2 b$ ). This may suggest that a small part of the difference in peripheral insulin sensitivity between young and old adults is related to lean mass. Insulin-mediated suppression of endogenous glucose production (\%EGP suppression, $\mathrm{p}=0.850$, Figure $2 \mathrm{c}$ ) was not different between 
young and old. RER in basal conditions was also comparable between older and young individuals $(p=0.174$, Figure $2 d)$. Likewise, upon insulin stimulation, RER was found to be equal between the older and young groups $(p=0.740$, Figure $2 d)$. As a result, the change in RER upon insulin stimulation (known as metabolic flexibility) was also not different between older and young individuals $(0.10(0.05)$ vs. 0.09 (0.03), respectively, $p=0.603)$.
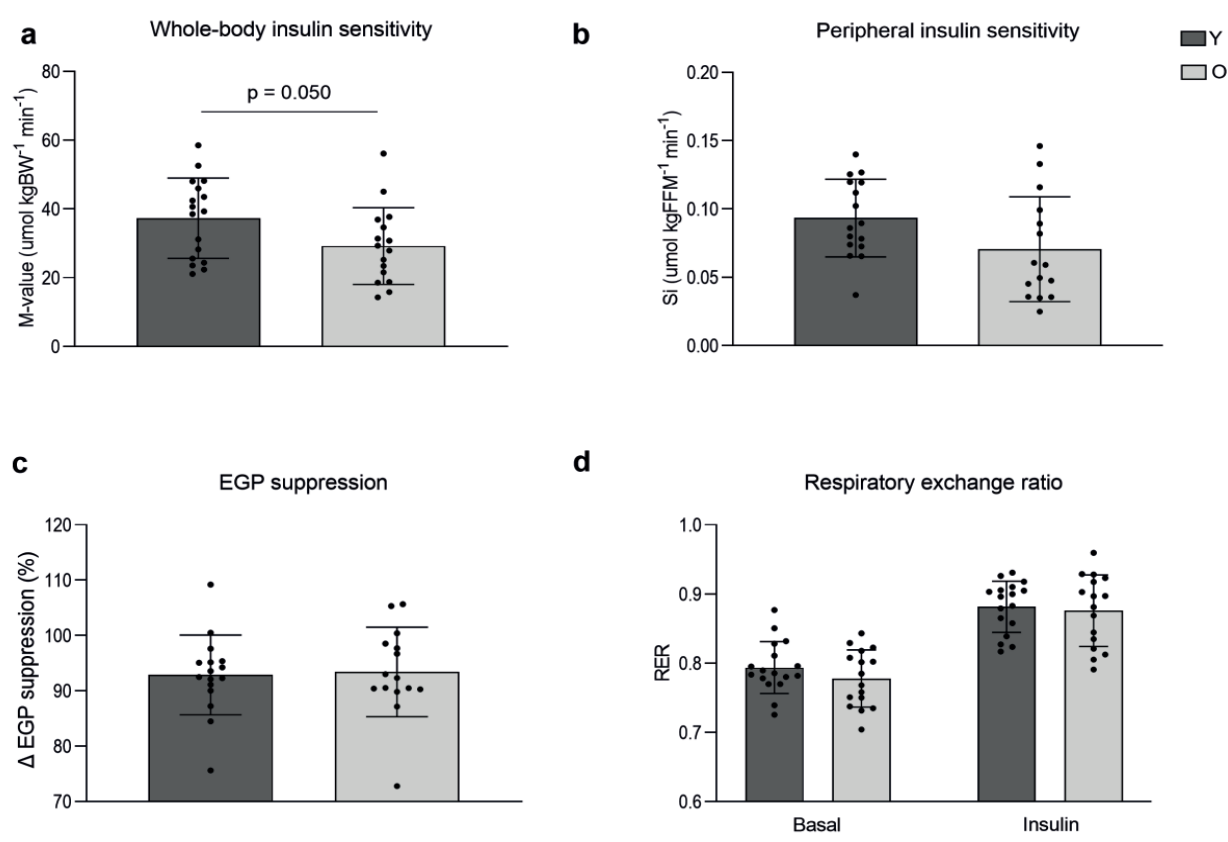

Figure 2 Effect of aging on insulin sensitivity and substrate selection. a Whole-body insulin sensitivity based on the M-value measured during a hyperinsulinemic-euglycemic clamp. $\mathbf{b}$ Insulin-stimulated glucose uptake, corrected for plasma insulin and glucose levels $\left(S_{i}\right)$ and expressed per $\mathrm{kg}$ FFM. c EGP suppression, calculated as the percentage insulinsuppressed EGP from the basal EGP d Respiratory exchange ratio measured before, in resting conditions (Basal), and during insulin stimulation (Insulin). Dark grey bars represent the young individuals $(Y, n=17)$, light grey bars represent older individuals $(O, n=15)$. One participant from $\mathrm{O}$ was excluded for analysis due to a violation of the protocol instructions. For one $O$ participant, tracer data could not be analyzed. Values are presented as mean \pm SD (with individual data points), $p=0.05$ denotes a significant difference between the two groups (two-sided, independent samples t-test). M-value = Mean glucose infusion rate; BW = body weight; $\mathrm{S}_{\mathrm{i}}=$ insulin-stimulated glucose disposal; FFM = fat-free mass; $\mathrm{EGP}=$ endogenous glucose production. 


\section{Less gait stability but comparable adaptability in older adults}

To further determine physical function, walking stability was examined via step variability during unperturbed walking trials at multiple speeds on a treadmill and via stability of the body configuration (margin of stability; MoS; (27)) in response to treadmill belt acceleration perturbations causing forward balance loss during walking (see Methods for protocol details). Compared to young, older participants had a larger step length variability during unperturbed walking $\left(F_{(1,30)}=7.077, p=0.012\right.$; significant pairwise comparisons at 1.2, 1.4, and $1.6 \mathrm{~m} \mathrm{~s}^{-1}$; Supplementary Figure 1), but no other significant differences were found. During the walking perturbation assessments, group comparisons for the entire perturbation trial revealed a significant difference in the margin of stability (MoS) during the first perturbation ( $F(1$, 28) $=7.7, p=0.010$ ) but not the second and ninth perturbations, indicating that older adults initially perform poorer than young adults, but the ability of older adults to adapt gait in response to repeated perturbations is intact (Supplementary Figure 2a). More detailed results regarding gait stability and stepping behavior can be found in the supplementary notes and accompanying figures.

\section{Lower ex vivo and in vivo mitochondrial function in older adults}

Skeletal muscle mitochondrial capacity was determined both ex vivo in permeabilized muscle fibers and in vivo via non-invasive assessment of $\mathrm{PCr}$ recovery rates using ${ }^{31} \mathrm{P}-\mathrm{MR}$ spectroscopy. In permeabilized muscle fibers, mitochondrial state 2 respiration (i.e., respiration in the presence of substrate alone) was similar between young and older adults on most substrate combinations, apart from malate + octanoyl-carnitine, which showed lower respiration rates in older individuals ( $\mathrm{MO}, \mathrm{p}=0.044$, Figure 3a). ADP-stimulated (state 3 ) respiration, fueled by complex I-linked substrates (malate + glutamate, MG, $p=0.032$, Figure $3 b$ ), was lower in older participants whereas state 3 respiration upon a lipid substrate (MO, Figure $3 c$ ) was not significantly different between young and old ( $p>0.05$ ). ADPstimulated respiration upon parallel electron input to both Complex I and II was also lower in older compared to young individuals. Thus, state 3 respiration upon malate + octanoyl-carnitine + glutamate (MOG, $p=0.014$, Figure $3 \mathrm{~d}$ ), malate + octanoylcarnitine + glutamate + succinate (MOGS, $p=0.006$, Figure $3 d$ ) and malate +

glutamate + succinate (MGS, $p=0.077$, Figure $6 \mathrm{~d}$ ) was $\sim 11-15 \%$ lower in the older adults as compared to the young individuals, although the latter did not reach 


\section{Chapter 3}

statistical significance. Maximal FCCP-induced uncoupled respiration, reflecting the maximal capacity of the electron transport chain, was $\sim 17 \%$ lower in older adults compared to young (State $3 u, p=0.008$, Figure 3e). State 40 respiration, reflecting mitochondrial proton leak, was similar between the young and older individuals $(p=$ 0.379 , Figure $3 f)$. The negligible increase in oxygen consumption upon cytochrome C (2.30 (3.24) vs. 2.27 (2.26) \% in young vs. old, respectively) underscores the viability and quality of the muscle fibers and was similar in both groups $(p=0.976)$.

Assessment of in vivo mitochondrial function revealed a $\sim 16 \%$ lower $\mathrm{PCr}$ recovery rate constant in older adults as compared to young ( $p=0.003$, Figure $3 g$ ), further confirming a decreased mitochondrial oxidative capacity in older individuals. The exercise-induced $\mathrm{PCr}$ depletion and end-exercise $\mathrm{pH}$ were similar between young and old $(\mathrm{p}>0.05)$ and $\mathrm{pH}$ remained above 6.9, indicating no substantial exerciseinduced acidification in either group.

To complement the in vivo and ex vivo mitochondrial function measurements, mitochondrial content was estimated by mitochondrial Oxidative Phosphorylation (OXPHOS) protein expression. However, young and older adults displayed a similar expression of each of the five OXPHOS proteins ( $p>0.05$, Figure $3 h$ ). 
a

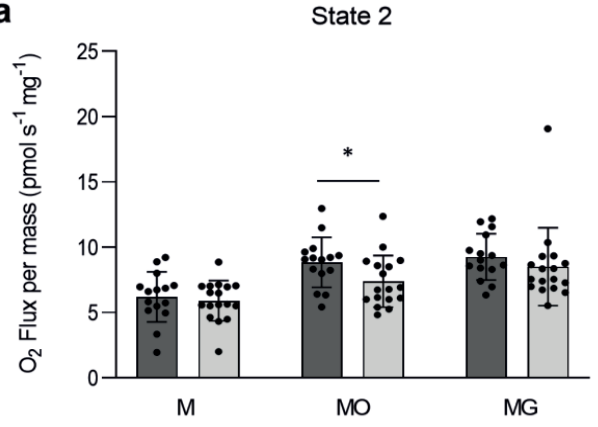

b

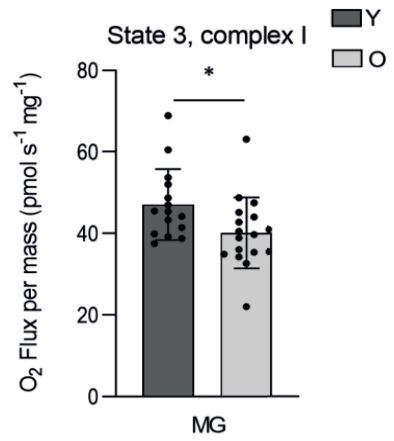

State 3, complex I+II c

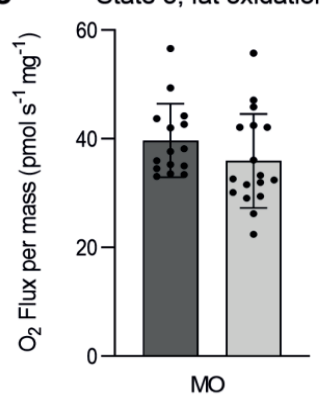

d

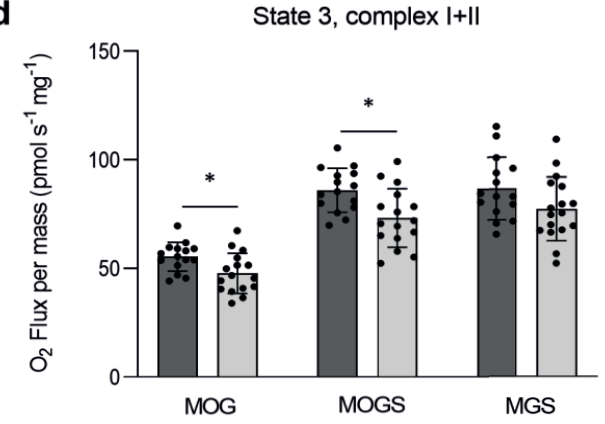

e State uncoupled, complex $1+\mid I$

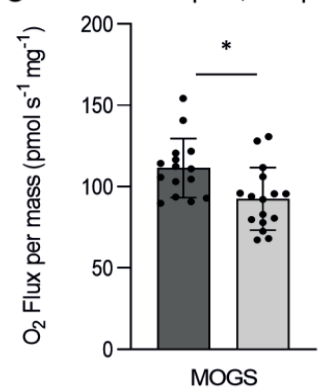

h

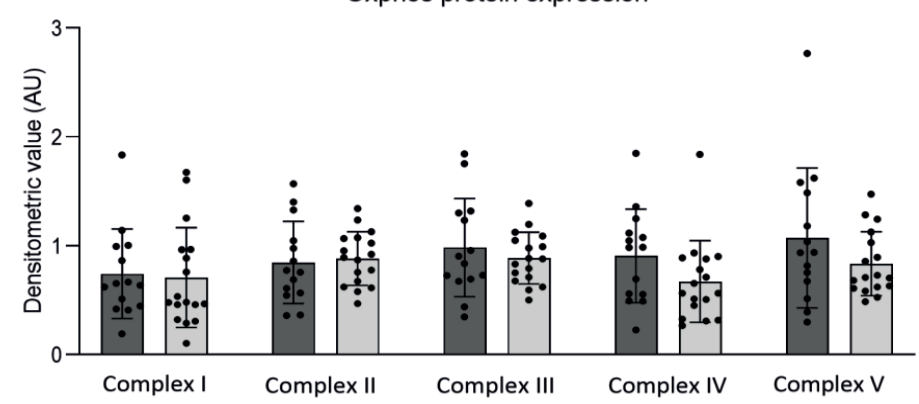

Figure 3 Effect of aging on skeletal muscle mitochondrial function and density 
Figure 3 Effect of aging on skeletal muscle mitochondrial function and density. a Mitochondrial respiration upon substrates only (state 2$)(\mathrm{Y}, \mathrm{n}=15$; $\mathrm{O}=17$ ). $\mathbf{b}$ ADPstimulated respiration (state 3 ) respiration fueled by Complex I-linked substrates $(Y, n=15$; $\mathrm{O}=17)$. c State 3 upon a lipid substrate $(Y, n=15 ; O=17)$. d Maximal ex vivo state 3 respiration upon parallel electron input into Complex II and I $(Y, n=15 ; O=17)$. e Maximal uncoupled respiration upon $\mathrm{FCCP}(\mathrm{Y}, \mathrm{n}=15 ; \mathrm{O}=17)$. $\mathrm{f}$ Mitochondrial respiration uncoupled from ATP synthesis (state 4o) ( $\mathrm{Y}, \mathrm{n}=15 ; \mathrm{O}=17$ ). $\mathbf{g}{ }^{31} \mathrm{P}-\mathrm{MRS}$ in vivo assessment of mitochondrial oxidative capacity $(\kappa)(\mathrm{Y}, \mathrm{n}=15 ; \mathrm{O}, \mathrm{n}=16)$. $\mathbf{h}$ Mitochondrial protein expression of oxidative phosphorylation (OXPHOS) complex I, complex II, complex III , complex IV, and complex $\mathrm{V}(\mathrm{Y}, \mathrm{n}=15 ; \mathrm{O}, \mathrm{n}=17)$. Dark grey bars represent the young individuals $(\mathrm{Y})$; light grey bars represent older individuals $(\mathrm{O})$. In one $\mathrm{Y}$ no biopsy was performed due to technical issues and, in another $Y$, due to the implications of the SARSCoV-19 outbreak. For the latter reason, also $\mathrm{PCr}$ recovery data is missing in the same $\mathrm{Y}$ participant. $\mathrm{PCr}$ data from one $\mathrm{O}$ has been excluded from analysis due to a $\mathrm{pH}$ decline below 6.9. $\mathrm{PCr}$ data from a $\mathrm{Y}$ participant has been excluded due to issues regarding the analysis. Values are presented as mean $\pm S D$ (with individual data points), * denotes significant differences between the two groups ( $p<0.05$, two-sided, independent samples $t-$ test). $\mathrm{M}=$ malate; $\mathrm{O}=$ octanoyl-carnitine; $\mathrm{G}=$ glutamate; $\mathrm{S}=$ succinate; $\kappa=$ phosphocreatine resynthesizes rate constant.

\section{Exercise training affects mitochondrial function and muscle health}

Next, we compared muscle function and mitochondrial capacity of the same older adults with normal PA $(\mathrm{O}, \mathrm{n}=17)$ to trained older adults $(\mathrm{TO}, \mathrm{n}=19)$ as well as to older adults with a low physical function ( $I O, n=6)$. Table 2 summarizes the main participant characteristics of these three older groups. Sex distribution was comparable across the groups $(p=0.982)$. Average age was 71 (4) years for the older participants with normal PA $(\mathrm{O})$ and 72 (5) years for the physically impaired older adults (IO), whereas TO were slightly younger at 68 (2) years in comparison with $O(p=0.023)$. In accordance with their training status, TO displayed a lower BMI and a lower body fat mass percentage as compared to both $O(p=0.046$ and $p$ $=0.050$, respectively) and $\mathrm{IO}$ ( $\mathrm{p}=0.024$ and $\mathrm{p}=0.021$, respectively). FFM was comparable $(p>0.05)$ across the three groups. TO displayed a higher average step count per day as compared to $O(p=0.050)$ and $I O(p=0.004)$, further underscoring their active lifestyle. TO also spent a significantly higher proportion of their waking time at a high intensity level of PA compared to $O(p=0.049)$ and $I O(p=0.005)$. Proportion of the waking time spent at a low intensity level of PA was similar between TO and $\mathrm{O}$ and between TO and $\mathrm{IO}(\mathrm{p}>0.05)$ and higher in $\mathrm{O}$ compared to IO $(p=0.038)$. As anticipated, IO performed significantly poorer on the chair-stand 
test as compared to $O(p=0.015)$ and TO $(p=0.001)$. Walking speed during the 4meter walk test was also lower in 10 when compared to TO $(p=0.009)$ but not to O $(p=0.227)$.

Table 2 Participant body composition, physical activity, and physical function.

\begin{tabular}{|c|c|c|c|}
\hline & $\begin{array}{l}\text { Older adults, } \\
\text { normal physical } \\
\text { activity (O) }\end{array}$ & $\begin{array}{l}\text { Trained older } \\
\text { adults (TO) }\end{array}$ & $\begin{array}{l}\text { Physically } \\
\text { impaired older } \\
\text { adults (IO) }\end{array}$ \\
\hline $\mathbf{N}$ & 17 & 19 & 6 \\
\hline Gender F/M ${ }^{\dagger}$ & $8 / 9$ & $8 / 11$ & $3 / 3$ \\
\hline Age (years) & $71(4)$ & $68(2)^{b}$ & $72(5)^{a, b}$ \\
\hline BMI $\left(\mathrm{kg} \mathrm{m}^{-2}\right)$ & $25.8(3.3)^{\mathrm{a}}$ & $23.6(1.9)^{\mathrm{b}}$ & $27.0(2.3)^{\mathrm{a}}$ \\
\hline Body weight (kg) & $74.0(11.9)$ & $68.3(8.8)$ & $79.0(7.2)$ \\
\hline Fat mass $(\%)$ & $33.1(9.2)$ & $26.2(7.7)^{\mathrm{b}}$ & $37.4(9.0)^{\mathrm{a}}$ \\
\hline Fat mass $(\mathrm{kg})$ & $24.5(7.9)^{\mathrm{a}}$ & $17.6(7.1)^{\mathrm{b}}$ & $29.4(7.9)^{\mathrm{a}}$ \\
\hline Fat free mass $(\mathrm{kg})$ & $49.5(10.8)$ & $50.7(9.7)$ & $49.6(10.8)$ \\
\hline Steps/day & $10,207(2,832)$ & $13,815(5,934)^{b}$ & $6,608(1,765)^{\mathrm{a}}$ \\
\hline HPA time ( $\%$ of wake time) & $2.2(1.2)^{\mathrm{a}}$ & $5.3(3.9)^{\mathrm{b}}$ & $1.0(0.6)^{\mathrm{a}}$ \\
\hline LPA time (\% of wake time) & $11.7(2.6)^{\mathrm{a}}$ & $10.8(2.9)$ & $8.3(1.5)^{b}$ \\
\hline SPPB $4 \mathrm{~m}$ walk speed $\left(\mathrm{m} \mathrm{s}^{-1}\right)$ & $1.1(0.2)$ & $1.3(0.2)^{\mathrm{a}}$ & $1.0(0.3)^{b}$ \\
\hline SPPB Chair-stand test (s) & $10.1(1.4)$ & $9.0(2.1)^{a}$ & $13.30(4.8)^{\mathrm{b}}$ \\
\hline
\end{tabular}

Values are presented as mean (SD). Age and HPA time were analyzed by two-sided Kruskal-Wallis tests followed by Bonferroni correction; other data were analyzed by one-way ANOVA with Tukey's post-hoc test. ${ }^{a, b}$ Groups that do not share the same letter are significantly different from each other $(p<0.05)$. ${ }^{\dagger}$ Sex distribution across groups was tested by $x^{2}$ tests $(p=0.982)$. BMI = body mass index; HPA = high-intensity physical activity; LPA = low-intensity physical activity; SPPB = short physical performance battery.

Higher muscle strength, volume, and endurance in trained older adults

During the 6MWT, IO covered $\sim 22 \%$ less distance compared to the $\mathrm{O}$ group $(\mathrm{p}=$ 0.013 , Figure $4 a$ ) and $\sim 29 \%$ less distance compared to the TO group ( $p=0.005$, Figure 4a). Not surprisingly, the trained older individuals displayed the highest cardiorespiratory fitness levels, as exemplified by an average $\mathrm{VO}_{2}$ max which was 1.2-fold and $\sim 1.4$-fold higher in comparison with $\mathrm{O}(\mathrm{p}=0.003)$ and $\mathrm{IO}(\mathrm{p}=0.004)$, respectively (Figure 4b). Accordingly, maximal power output was higher in TO as compared to $\mathrm{O}$ and $\mathrm{IO}$ ( $\mathrm{p}<0.001$ and $\mathrm{p}<0.001$ respectively, Figure 4c). TO participants also showed the highest muscle strength, whereas muscle strength was lowest in the physically impaired older adults. Thus, isokinetic extension peak torque (Figure $4 d$ ) was $\sim 24 \%$ and $\sim 27 \%$ lower in the 10 group as compared to $O$ ( $p=$ $0.026)$ and TO $(p=0.006)$, respectively. Furthermore, the isokinetic flexion peak 


\section{Chapter 3}

torque (Figure 4d) was found to be highest in the TO group compared to the $\mathrm{O}$ group $(\sim 1.1$-fold, $p=0.046)$ and $\mathrm{IO}$ group $(\sim 1.5$-fold, $\mathrm{p}<0.001)$. Muscle volume, measured in the upper leg by MRI, was not significantly different between the three groups ( $p>$ 0.05 , Figure $4 \mathrm{e})$, which was in accordance with the observed FFM. 
Aging and Skeletal Muscle and Mitochondrial Health
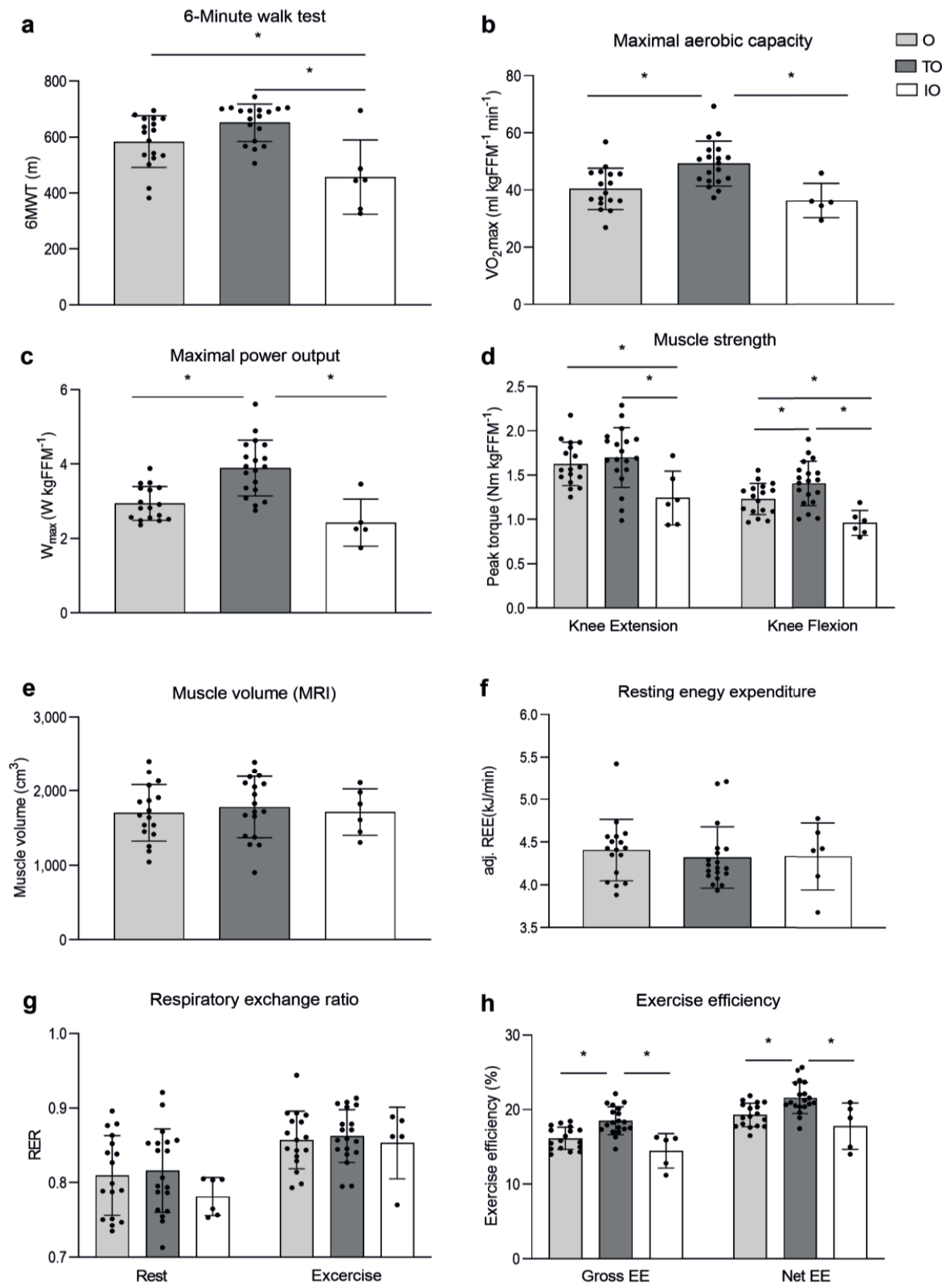

Figure 4 Effect of exercise training and physical impairment on muscle health and exercise efficiency in older adults. 
Figure 4 Effect of exercise training and physical impairment on muscle health and exercise efficiency in older adults. a Walking distance during the 6MWT performed on the Carensystem $(\mathrm{O}, \mathrm{n}=17 ; \mathrm{TO}, \mathrm{n}=17 ; \mathrm{IO}, \mathrm{n}=6)$. $\mathbf{b}$ Maximum rate of oxygen consumption measured during graded cycling exercise $(O, n=17 ; \mathrm{TO}, \mathrm{n}=19 ; \mathrm{IO}, \mathrm{n}=6)$. c Maximal power output measured during graded cycling test $(\mathrm{O}, \mathrm{n}=17 ; \mathrm{TO}, \mathrm{n}=19 ; \mathrm{IO}, \mathrm{n}=6)$. d Muscle strength expressed as the extension and flexion peak torque during an isokinetic protocol on the Biodex system and corrected for fat-free mass $(\mathrm{O}, \mathrm{n}=17 ; \mathrm{TO}, \mathrm{n}=19 ; \mathrm{IO}, \mathrm{n}=$ 6). e Upper leg muscle volume measured by $\mathrm{MRI}(\mathrm{O}, \mathrm{n}=17 ; \mathrm{TO}, \mathrm{n}=18 ; \mathrm{IO}, \mathrm{n}=6)$. $\mathbf{f}$ Resting energy expenditure adjusted for FFM (O, $\mathrm{n}=17 ; \mathrm{TO}, \mathrm{n}=19 ; \mathrm{IO}, \mathrm{n}=5)$. $\mathbf{g}$ Respiratory exchange rate measured before, in resting conditions, and during submaximal exercise $(O, n=17 ; T O, n=19 ; 1 O, n=5)$. $\mathbf{h}$ Exercise efficiency measured during a submaximal cycle test and expressed as gross efficiency and net efficiency $(O, n=17 ; T O, n$ $=19 ; \mathrm{IO}, \mathrm{n}=5$ ). Light grey bars represent normally active older adults $(\mathrm{O})$; dark grey bars represent trained older adults (TO); white bars represent physically impaired older adults (IO). Upon the advice of the responsible medical doctor, one IO participant did not perform a maximal and submaximal. One TO participant did not perform the 6MWT due to scheduling issues. The reported 6MWT distance from another TO was invalid and therefore excluded for analysis. Data presented in panels a and $c$ were analyzed by two-sided Kruskal-Wallis tests followed by Bonferroni correction. Data presented in panels $b$, d-h were analyzed by one-way ANOVA with Tukey's post-hoc test. Values are presented as mean \pm SD (with individual data points), ${ }^{*}$ denotes significant differences between two groups $(p<0.05)$. $6 \mathrm{MWT}=6$-minute walk test distance; $\mathrm{VO}_{2}$ max $=$ maximal aerobic capacity; $\mathrm{Nm}=$ newton meters; FFM = fat-free mass; RER = respiratory exchange rate; $\mathrm{REE}$ = resting energy expenditure; $\mathrm{EE}=$ exercise efficiency.

\section{Higher exercise efficiency in trained older adults}

Resting energy expenditure was comparable across the three older adult groups ( $p>$ 0.05, Figure 4f) and RER was also similar between the groups ( $p>0.05$, Figure 4g). Furthermore, during the 1-hour submaximal cycling test at $50 \%$ of $W_{\max }$, no differences were observed in RER in TO as compared to $\mathrm{O}$ and $\mathrm{IO}$ ( $p>0.05$, Figure $4 g)$. TO showed a higher gross exercise efficiency in comparison to $O(p=0.001)$ and in comparison with 10 ( $p<0001$, Figure 4h) Likewise, the net exercise efficiency was higher in TO compared to both the $O$ individuals $(p=0.006)$ and the IO group $(p$ $=0.002$, Figure 4h) .

\section{Higher whole-body insulin sensitivity in trained older adults}

Glucose infusion rate during the clamp (M-value) was 1 .3-fold higher in TO when compared to $\mathrm{O}(\mathrm{p}=0.023$, Figure 5a), indicating a higher whole-body insulin sensitivity in TO. Peripheral insulin sensitivity $\left(\mathrm{S}_{\mathrm{i}}\right)$ was also higher in TO compared to $\mathrm{O}$ when expressed per $\mathrm{kg}$ body weight, which tended to be significant (TO: 0.066 (0.025) $\mu \mathrm{mol} \mathrm{kgBW} \mathrm{min}^{-1}$ vs. O: 0.050 (0.028) $\mu \mathrm{mol} \mathrm{kgBW} \mathrm{min}^{-1}$, respectively, $\mathrm{p}=$ 
0.072). When $\mathrm{S}_{i}$ was corrected for FFM, no significant difference was observed between TO and $\mathrm{O}(\mathrm{p}=0.165$, Figure $5 \mathrm{~b})$. Insulin-mediated suppression of EGP (Figure 5c) was similar in TO and O. Also RER was comparable between TO and O, both in the basal state $(p=0.839$, Figure $5 d)$ and upon insulin stimulation $(p=0.353$, Figure $5 \mathrm{~d}$ ). As a result, metabolic flexibility was also comparable between $\mathrm{TO}$ and $\mathrm{O}$ (0.11 (0.04) vs. $0.10(0.05)$, respectively, $p=0.261)$.

a

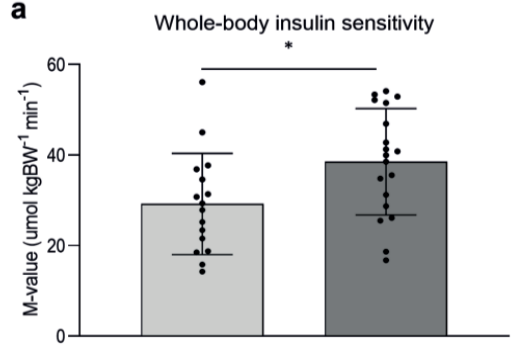

C

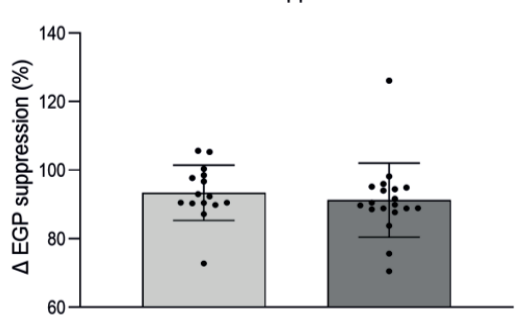

b

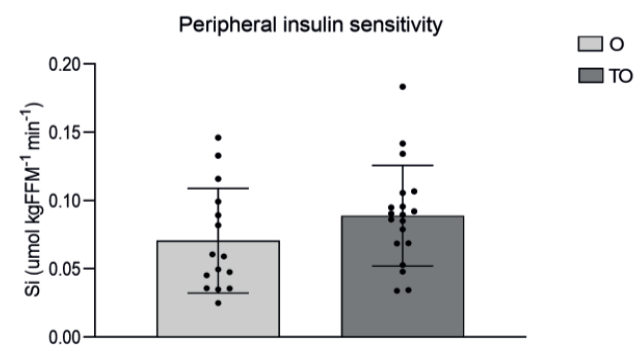

d Respiratory exchange ratio

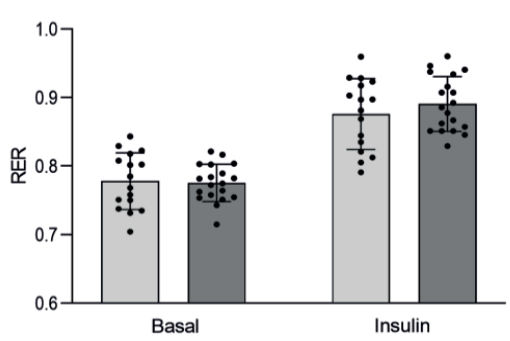

Figure 5 Effect of exercise training on insulin sensitivity and substrate selection in older adults. a Whole-body insulin sensitivity based on the M-value measured during a hyperinsulinemic-euglycemic clamp. b Insulin-stimulated glucose uptake corrected for plasma insulin and glucose levels $\left(S_{i}\right)$ and expressed per kg FFM c EGP suppression, calculated as the percentage insulin-suppressed EGP from the basal EGP. d Respiratory exchange ratio measured before, in resting conditions (Basal), and during insulin stimulation (Insulin). Light grey bars represent normally active older adults $(O, n=15)$; dark grey bars represent trained older adults (TO, $n=19$ ). Because only 3 IO participants could undergo the clamp, these results were not considered. One $O$ participant was excluded for analysis due to violation of the protocol instructions. For one $O$ participant tracer data could not be analyzed. Values are presented as mean \pm SD (with individual data points), * denotes significant differences between the two groups $(p<0.05$ two-sided, independent samples ttest). $\mathrm{M}$-value $=$ Mean glucose infusion rate; $\mathrm{BW}=$ body weight; $\mathrm{S}_{\mathrm{i}}=$ insulin-stimulated glucose disposal; FFM = fat-free mass; EGP = endogenous glucose production. 


\section{Comparable gait stability in normally active and trained older adults}

When comparing $\mathrm{O}$, TO and $\mathrm{IO}$, no significant differences were observed for gait variability during unperturbed walking (Supplementary Figure 3 and 4). During the gait stability assessments, when considering the entire perturbation trial (Supplementary Figure 5a), MoS was not significantly different between $\mathrm{O}$ and TO for perturbation 1, 2 and 9, indicating no significant stability or stepping behavior difference between $\mathrm{O}$ and $\mathrm{TO}$. More detailed results regarding the gait variability, gait stability, and adaptability can be found in the supplementary notes and accompanying figures.

\section{Higher mitochondrial function in trained older adults}

In permeabilized muscle fibers, mitochondrial state 2 respiration was significantly higher in the TO individuals compared to $\mathrm{O}$ on all of the substrate combinations studied (Figure 6a, $\mathrm{p}<0.05$ ). Also, ADP-stimulated (state 3 ) respiration on complex I-linked substrates as well as a lipid substrate was higher in TO compared to $\mathrm{O}$. Thus, state 3 respiration fueled by malate + glutamate (MG) and malate + octanoylcarnitine $(\mathrm{MO})$ were $\sim 1.2$-fold and $\sim 1$.4-fold higher in TO as compared to O $(p=0.049$ and $p<0.001$, respectively (Figure 6b-c). ADP-stimulated respiration upon parallel electron input to both Complex I and II was also higher in TO compared to $\mathrm{O}$. Thus, state 3 respiration upon malate + octanoyl-carnitine + glutamate $(\mathrm{MOG}, \mathrm{p}$ $=0.001)$, malate + octanoyl-carnitine + glutamate + succinate (MOGS, $p=0.002)$ and malate + glutamate + succinate (MGS, $p=0.018$ ) was $\sim 1.2-1.3$-fold higher in TO compared to $\mathrm{O}$ (Figure $6 \mathrm{~d}$ ). Maximal FCCP-induced uncoupled respiration was 1.4-fold higher in TO compared to $\mathrm{O}$ (state $3 \mathrm{u}, \mathrm{p}=0.001$, Figure $6 \mathrm{e}$ ). State 40 (leak) respiration was higher in the TO group compared to both $O(\sim 1.3$-fold, $p=$ 0.006 , Figure 14f) and $1 \mathrm{O}$ individuals ( 1.3-fold higher, $p=0.026$, Figure $6 f)$. Across the different respiratory states, oxygen consumption in permeabilized muscle fibers derived from $1 \mathrm{O}$ appeared to be comparable to the respiration rates observed in $\mathrm{O}$. However, due to the small sample size and large variation of the IO group, the differences between the $\mathrm{IO}$ and $\mathrm{TO}$ groups did not reach statistical significance in all respiratory states except for state 40.

The negligible increase in oxygen consumption upon cytochrome C $(2.61$ (1.85) \% vs. 2.27 (2.26) \% vs. 0.33 (1.54) \%, in TO vs. O vs. IO, respectively) underscores the 
viability and quality of the muscle fibers and was similar between the study groups ( $p$ $>0.05)$.

Similar to the observed mitochondrial respiration rates in permeabilized muscle fibers, the TO group also displayed a $\sim 1$.2-fold and $\sim 1$.3-fold higher PCr recovery rate constant as compared to $\mathrm{O}$ and $\mathrm{IO}$, respectively, although these differences did not reach statistical significance (TO vs. O $p=0.169 ;$ TO vs. OI, $p=0.135$; O vs. IO $\mathrm{p}=0.815$; Figure $6 \mathrm{~g}$ ). The exercise-induced $\mathrm{PCr}$ depletion and $\mathrm{pH}$ was similar across the three study groups $(\mathrm{p}>0.05)$ and $\mathrm{pH}$ remained above 6.9 indicating no substantial exercise-induced acidification.

Protein expression of OXPHOS complex I and III was higher in TO compared to O ( $p$ $=0.010$ and $p=0.042$, respectively, Figure 6h). Furthermore, protein expression of complex II ( $p=0.003$ and $p=0.025$, respectively) and complex IV $(p<0.001$ and $p=$ 0.003 , respectively) was higher in TO compared to both $\mathrm{O}$ and $\mathrm{IO}$. 

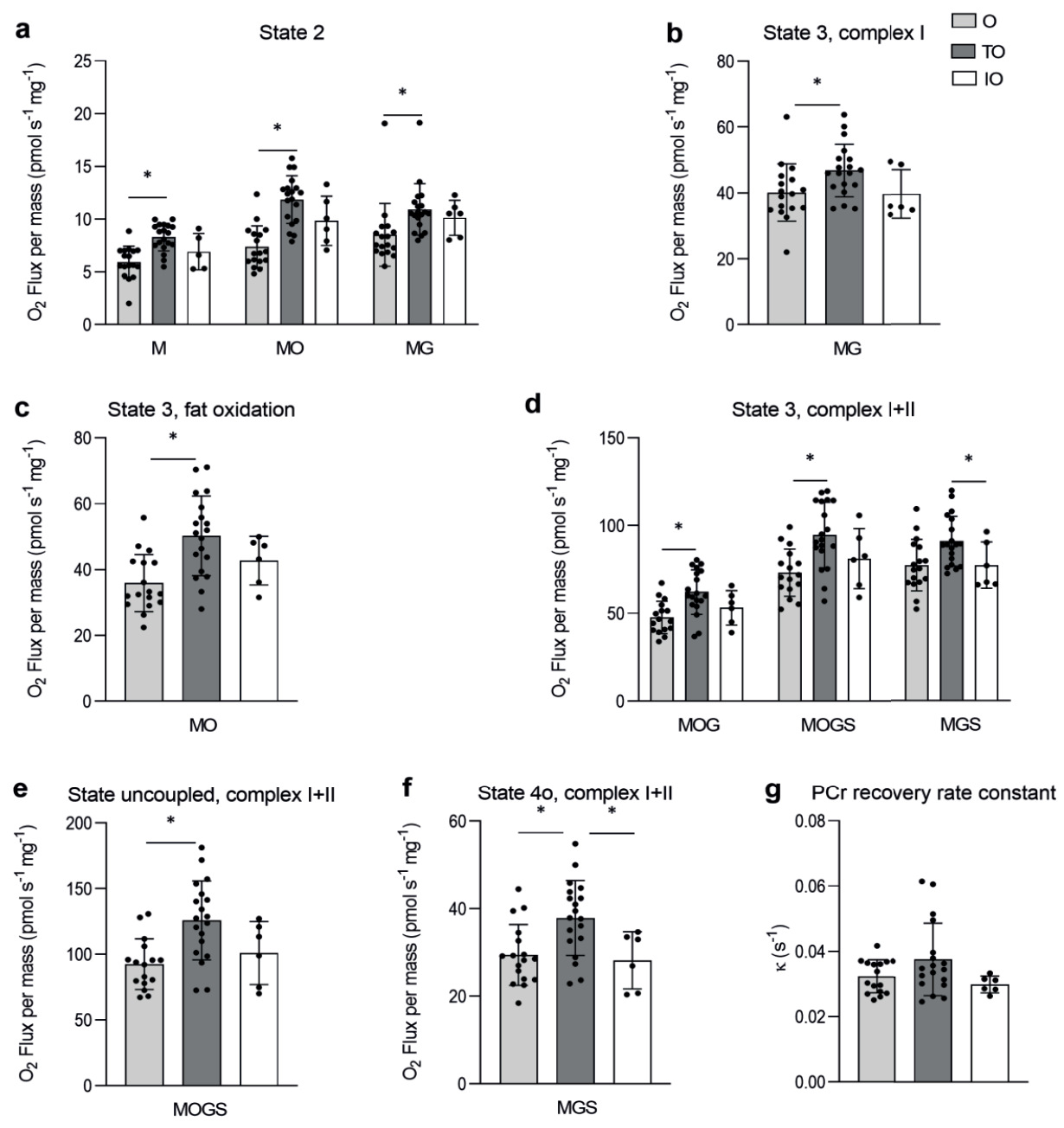

h

Oxphos protein expression

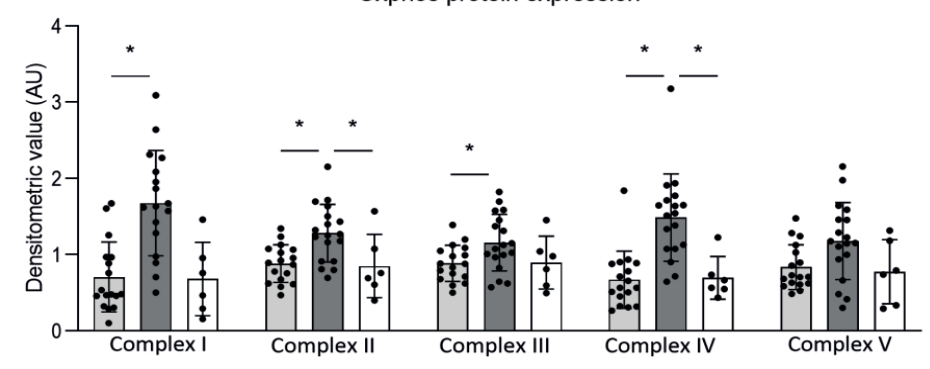

Figure 6 Effect of exercise training and physical impairment on skeletal muscle mitochondrial respiration and density in older adults. 
Figure 6 Effect of exercise training and physical impairment on skeletal muscle mitochondrial respiration and density in older adults. a Mitochondrial respiration upon substrates only (state 2$)(\mathrm{O}, \mathrm{n}=17 ; \mathrm{TO}, \mathrm{n}=19 ; \mathrm{IO}, \mathrm{n}=6)$. b ADP-stimulated respiration (state 3 ) fueled by Complex I-linked substrates (O, $\mathrm{n}=17 ; \mathrm{TO}, \mathrm{n}=19 ; \mathrm{IO}, \mathrm{n}=6)$ ). c State 3 respiration upon a lipid substrate $(\mathrm{O}, \mathrm{n}=17$; TO, $\mathrm{n}=19 ; \mathrm{IO}, \mathrm{n}=6)$. d Maximal ex vivo state 3 respiration upon parallel electron input into Complex I and II $(\mathrm{O}, \mathrm{n}=17$; TO, $\mathrm{n}=19$; IO, $\mathrm{n}=$ 6). e Maximal uncoupled respiration upon $\operatorname{FCCP}(\mathrm{O}, \mathrm{n}=17 ; \mathrm{TO}, \mathrm{n}=19 ; \mathrm{IO}, \mathrm{n}=6)$. $\mathbf{f}$ Mitochondrial respiration uncoupled from ATP synthesis (state 4o) $(\mathrm{O}, \mathrm{n}=17$; TO, $\mathrm{n}=19$; $\mathrm{IO}, \mathrm{n}=6)$. $\mathbf{g}$ In vivo ${ }^{31} \mathrm{P}-\mathrm{MRS}$ estimate of mitochondrial oxidative capacity $\mathrm{k}(\mathrm{O}, \mathrm{n}=16$; TO, $\mathrm{n}$ $=18 ; \mathrm{IO}, \mathrm{n}=6)$. $\mathbf{h}$ Mitochondrial protein expression of oxidative phosphorylation (OXPHOS) complex I, complex II, complex III, complex IV, and complex V (O, n = 17; TO, n = 18; IO, $\mathrm{n}$ $=6$ ). Light grey bars represent normally active older adults $(O)$; dark grey bars represent trained older adults (TO); white bars represent physically impaired older adults (IO). $\mathrm{PCr}$ data from one $\mathrm{O}$ participant has been excluded from analysis due to a $\mathrm{pH}$ decline below 6.9. PCr from a TO participant has been excluded due to issues regarding the analysis. From one TO not enough muscle tissue was available for the OXPHOS analysis. Data presented in panels a and $\mathrm{g}$ were analyzed by two-sided Kruskal-Wallis tests followed by Bonferroni correction. Data presented in panels $b-f$ and $h$ were analyzed by one-way ANOVA with Tukey's post-hoc test. Values are presented as mean \pm SD (with individual data points), * denotes significant differences between two groups $(p<0.05)$. $M=$ malate; $O=$ octanoylcarnitine; $\mathrm{G}=$ glutamate; $\mathrm{S}=$ succinate; $\kappa=$ phosphocreatine resynthesis rate constant

\section{Mitochondrial and muscle function and exercise efficiency are related}

To further explore the relationship between mitochondrial energetics, muscle quality and physical function, correlations were performed based on the combined data from the entire study cohort. Pearson correlation outcomes are summarized in Supplementary Table 2 and illustrated in Figure 7. As anticipated, correlations were observed between the different measures for mitochondrial function and oxidative capacity (ex vivo respiration, in vivo $\mathrm{PCr}$ recovery rate and $\mathrm{VO}_{2} \mathrm{max}$, Figure $7 \mathrm{a}$ ). Furthermore, measures for ex vivo mitochondrial capacity - but not in vivo $\mathrm{PCr}$ recovery rate constant - were found to significantly correlate with 6MWT, M-value and steps per day as markers for walking performance, insulin sensitivity, and physical activity, respectively (Supplementary Table 2, Figure 7b-c). Both step length variability and double support time variability correlated with measures of in vivo and ex vivo mitochondrial function (Supplementary Table 2, Figure 7e-f). Additionally, ex vivo mitochondrial coupled respiration rates were found to correlate significantly with gross exercise efficiency (Supplementary Table 2, Figure 7d). Interestingly, gross and net exercise efficiency showed the strongest correlations with multiple aspects of muscle health including endurance, physical function, muscle strength, insulin 


\section{Chapter 3}

sensitivity, gait stability, and physical activity, as illustrated by the significant correlations with $\mathrm{VO}_{2}$ max, 6MWT, the chair-stand test, isokinetic extension, M-value, $\mathrm{S}_{\mathrm{i}}$, recovery steps after perturbation, and steps per day (Supplementary Table 2, Figure $7 \mathrm{~g}-\mathrm{h}$ ), respectively.

Partial correlation analyses adjusted for age, sex, and BMI are shown in Table 3. Also, after adjustment, correlations were observed between the different measures for mitochondrial function and oxidative capacity (ex vivo respiration, in vivo $\mathrm{PCr}$ recovery rate and $\mathrm{VO}_{2} \mathrm{max}$ ).

Interestingly, maximal ex vivo mitochondrial respiration rates were found to significantly correlate with steps per day, a marker for PA. In addition, ex vivo mitochondrial capacity - but not in vivo $\mathrm{PCr}$ recovery rate constant - were significantly correlated with gait stability assessed by double step support variability and insulin sensitivity as assessed by the M-value. Moreover, ex vivo mitochondrial coupled respiration rates were found to correlate significantly with gross exercise efficiency. Interestingly, gross and net exercise efficiency showed, also after adjustment, the strongest correlations with multiple aspects of muscle health including endurance, physical function, insulin sensitivity, and PA. The correlation between exercise efficiency and step variability was no longer present after adjustment for age and BMI. 
a

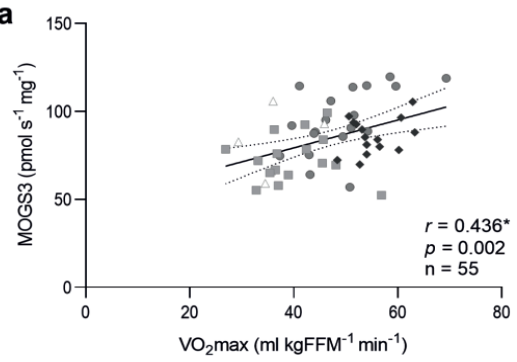

C

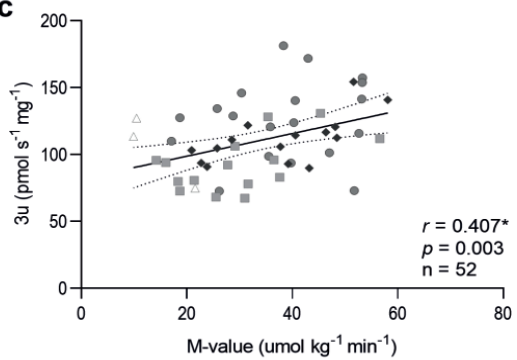

e
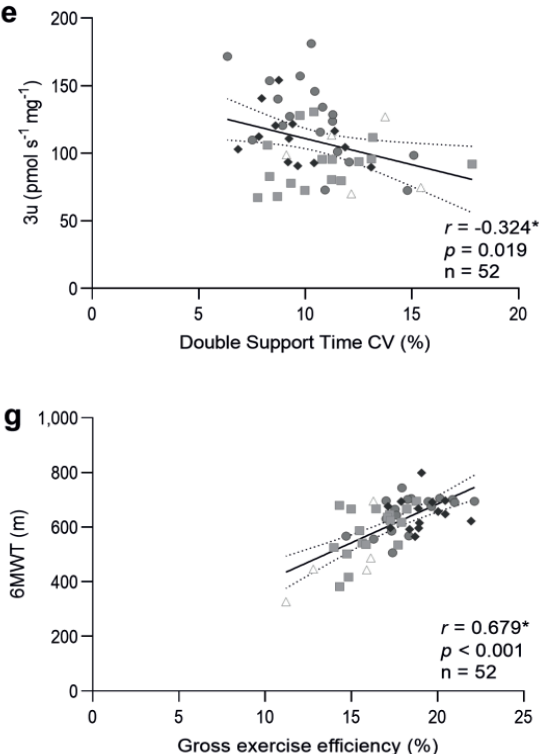

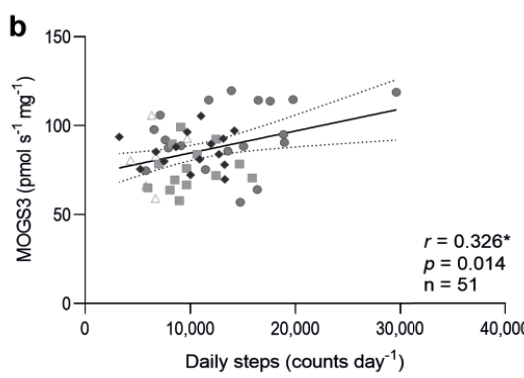

- $\mathrm{Y}$

- $\mathrm{O}$

$\triangle 10$

- TO
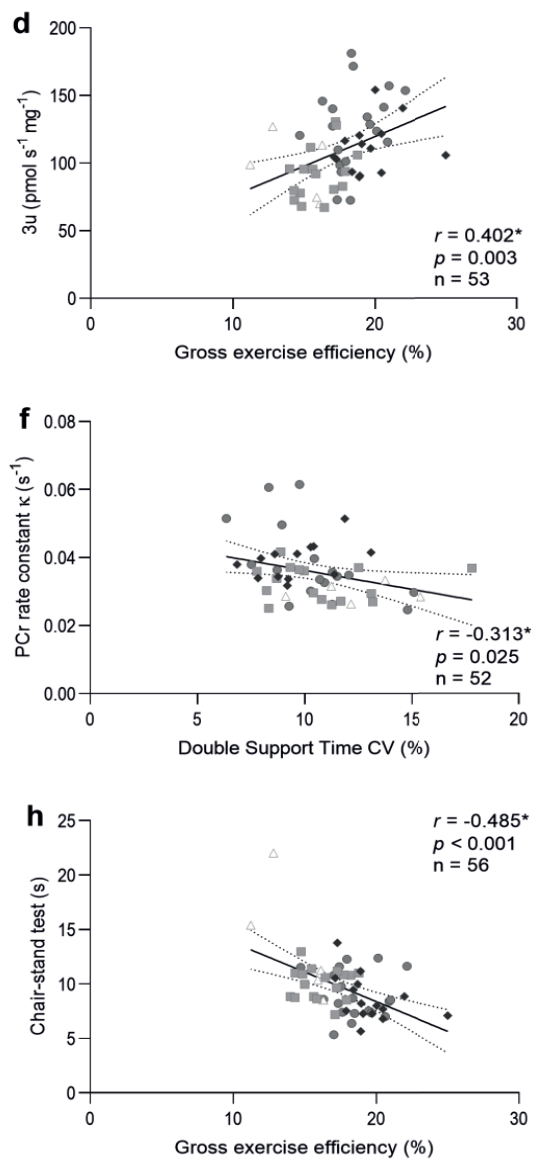

Figure 7 Bivariate correlations between mitochondrial function and physical muscle function. 
Figure 7 Bivariate correlations between mitochondrial function and physical muscle function. a Correlation between maximal exercise capacity and maximal coupled mitochondrial respiration. b Correlation between physical activity and maximal coupled mitochondrial respiration. c Correlation between whole-body insulin sensitivity and maximal uncoupled mitochondrial respiration. d Correlation between gross exercise efficiency and maximal uncoupled mitochondrial respiration. e Correlation between step variability and maximal uncoupled mitochondrial respiration $\mathbf{f}$ Correlation between step variability and in vivo $\mathrm{PCr}$ recovery rate constant. g Correlation between walking performance and gross exercise efficiency. $\mathbf{h}$ Correlation between SPPB chair-stand test and gross exercise efficiency. Dark grey diamonds indicate young normally active individuals ( $Y$ ), light grey squares indicate normally active older adults $(\mathrm{O})$, white triangles indicate physically impaired older adults individuals (IO), and dark grey circles indicate trained older adults (TO). * indicates the correlation is significant at the 0.05 level (2-tailed, $p<0.05$ ); Best-fit trend line and $95 \%$ confidence intervals are included. MOGS3 $=$ state 3 respiration upon malate + octanoylcarnitine + glutamate + succinate; $3 u=$ state 3 uncoupled respiration upon FCCP; $\kappa=$ phosphocreatine resynthesis rate constant; $6 \mathrm{MWT}=6$-minute walk test; $\mathrm{VO}_{2} \mathrm{max}=$ maximal aerobic capacity; FFM = fat-free mass; $\mathrm{Nm}=$ newton meters; $\mathrm{M}$-value = Mean glucose infusion rate; $r=$ Pearson correlation coefficient 
Table 3 Partial analysis to explore the relationships between energetics and physical muscle function

\begin{tabular}{|c|c|c|c|c|c|c|c|c|c|c|c|c|c|}
\hline Measurement & Variables & & $\begin{array}{l}\text { 6MWT } \\
(\mathrm{m})\end{array}$ & $\begin{array}{l}{ }^{\dagger} \text { Chair- } \\
\text { stand test } \\
\text { (s) }\end{array}$ & $\begin{array}{l}\mathrm{VO}_{2} \max \\
\left(\mathrm{ml} \mathrm{min}^{-1}\right. \\
\left.\mathrm{kgFFM}^{-1}\right)\end{array}$ & $\begin{array}{l}\text { Isokinetic } \\
\text { extension } \\
(\mathrm{Nm} \\
\left.\mathrm{kgFFM}^{-1}\right)\end{array}$ & $\begin{array}{l}\text { Isokinetic } \\
\text { flexion } \\
(\mathrm{Nm} \\
\left.\mathrm{kgFFM}^{-1}\right)\end{array}$ & $\begin{array}{l}\text { M-value } \\
\left(\mu \mathrm{mol} \mathrm{kg}^{-1}\right. \\
\left.\mathrm{min}^{-1}\right)\end{array}$ & $\begin{array}{l}\mathrm{S}_{\mathrm{i}} \\
(\mu \mathrm{mol} \\
\mathrm{kgFFM}^{-1} \\
\left.\min ^{-1}\right)\end{array}$ & $\begin{array}{l}\text { Daily } \\
\text { steps } \\
\text { (counts } \\
\text { day }^{-1} \text { ) }\end{array}$ & $\begin{array}{l}\text { \#Step } \\
\text { length } \\
\text { variability }\end{array}$ & $\begin{array}{l}\text { \#Double } \\
\text { support } \\
\text { time } \\
\text { variability }\end{array}$ & $\begin{array}{l}\text { Gross } \\
\text { efficiency } \\
(\%)\end{array}$ \\
\hline \multirow[t]{6}{*}{$\begin{array}{l}\text { Mitochondrial } \\
\text { function }\end{array}$} & $\begin{array}{l}\text { Maximal coupled } \\
\text { respiration }\end{array}$ & $r$ & 0.165 & -0.084 & $0.416^{*}$ & 0.049 & -0.054 & 0.253 & 0.062 & $0.363^{*}$ & -0.094 & $-0.293^{*}$ & 0.232 \\
\hline & $\begin{array}{l}\text { MOGS3 } \\
\left(\mathrm{pmol} \mathrm{s}^{-1} \mathrm{mg}^{-1}\right)\end{array}$ & $\mathrm{p}$ & 0.256 & 0.548 & 0.002 & 0.727 & 0.699 & 0.080 & 0.673 & 0.009 & 0.534 & 0.048 & 0.101 \\
\hline & $\begin{array}{l}\text { Maximal } \\
\text { uncoupled }\end{array}$ & $r$ & 0.109 & -0.140 & $0.512^{*}$ & 0.077 & -0.036 & $0.317^{*}$ & 0.169 & $0.422^{*}$ & -0.170 & $-0.378^{*}$ & $0.304^{*}$ \\
\hline & $\begin{array}{l}\text { respiration } \\
\left(\mathrm{pmol} \mathrm{s}^{-1} \mathrm{mg}^{-1}\right)\end{array}$ & $\mathrm{p}$ & 0.458 & 0.318 & $<0.001$ & 0.584 & 0.798 & 0.026 & 0.250 & 0.002 & 0.258 & 0.010 & 0.030 \\
\hline & $\begin{array}{l}\mathrm{PCr} \text { recovery } \\
\text { rate constant } \kappa\end{array}$ & $r$ & 0.191 & -0.037 & $0.363^{*}$ & 0.035 & -0.024 & 0.238 & 0.124 & $0.305^{*}$ & -0.216 & -0.271 & 0.233 \\
\hline & $\left(\mathrm{s}^{-1}\right)$ & $p$ & 0.194 & 0.795 & 0.009 & 0.807 & 0.866 & 0.103 & 0.406 & 0.032 & 0.150 & 0.068 & 0.103 \\
\hline \multirow[t]{4}{*}{$\begin{array}{l}\text { Exercise } \\
\text { efficiency }\end{array}$} & $\begin{array}{l}\text { Gross efficiency } \\
(\%)\end{array}$ & $r$ & $0.597^{*}$ & $-0.478^{*}$ & $0.570^{*}$ & 0.110 & 0.052 & $0.424^{*}$ & 0.224 & 0.361 & -0.132 & -0.152 & \\
\hline & & $\mathrm{p}$ & $<0.001$ & $<0.001$ & $<0.001$ & 0.434 & 0.710 & 0.002 & 0.121 & 0.009 & 0.383 & 0.315 & \\
\hline & $\begin{array}{l}\text { Net efficiency } \\
(\%)\end{array}$ & $r$ & $0.521^{*}$ & $0.418^{*}$ & $0.470^{*}$ & 0.003 & -0.040 & $0.397^{*}$ & 0.235 & 0.273 & -0.103 & -0.215 & \\
\hline & & $p$ & $<0.001$ & 0.002 & $<0.001$ & 0.983 & 0.778 & 0.004 & 0.104 & 0.052 & 0.494 & 0.152 & \\
\hline
\end{tabular}

Data are from all groups (total $n>51$ ). Correlations are adjusted for ${ }^{\dagger}$ age, sex, and BMI.* indicates the partial correlation is significant at the 0.05 level (two-sided, $\mathrm{p}<0.05$ ); ${ }^{\dagger}$ indicates variables that were log transformed; ${ }^{*}$ indicates the correlations corrected for only ${ }^{\dagger}$ age and BMI.

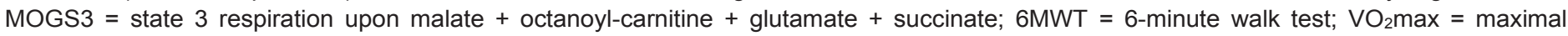
oxygen flow; FFM = fat-free mass; $\mathrm{Nm}=$ newton meters; $\mathrm{M}$-value $=$ mean glucose infusion rate; $\mathrm{S}_{\mathrm{i}}=$ insulin-stimulated glucose uptake, $\mathrm{r}=$ partial correlation coefficient. 


\section{DISCUSSION}

Skeletal muscle mitochondrial function has been shown to decline with age and may underlie the decline in muscle health and performance during aging. Although the origins of reduced mitochondrial function with age are a matter of ongoing debate, recent data suggest that PA levels may be a key determinant of mitochondrial energetics in aging (6). Although the data presented here support a role for PA in mitigating the negative effects of sedentary behavior in older adults, we nevertheless show that older adults with normal PA when compared to young adults with equivalent habitual PA levels, display lower mitochondrial capacity despite comparable mitochondrial content. Additionally, the older participants had lower muscle strength, aerobic capacity, exercise efficiency, and gait stability compared to similarly active young adults. Conversely, trained older participants exhibited higher mitochondrial capacity than older adults with normal PA and older adults with impaired physical function, which was associated with improved functional outcomes. Notably, the increase in mitochondrial capacity in the trained older adults was largely accounted for by an increase in mitochondrial content. These data suggest a role for aging-associated reductions in mitochondrial function independent of PA levels or mitochondrial content and may be a contributory factor in physical function decline. These effects appear to be mitigated by exercise training-induced increases in mitochondrial content.

In this study, the young and older adult groups were not engaged in structured exercise activities and both recorded approximately 10,000 steps daily. This is well above the average daily step count reported for older untrained adults in previous studies $(6,28)$ and indicates an overall active lifestyle and adequate levels of PA for a healthy adult (29). Despite similar habitual PA, walking performance and maximal aerobic capacity was lower in the older adults compared to the young controls. These findings are in line with previous observation from a study comparing adults < 65 years and $>65$ years, which observed the peak $\mathrm{VO}_{2}$ and $400 \mathrm{~m}$ gait speed to be lower with higher age (5).

With an average of more than 12,500 steps per day and at least 3 exercise sessions per week, the trained older participants in the current study are considered to be 
highly physically active, whereas physically impaired older adults averaged less than 7,500 steps per day, reflecting a sedentary lifestyle (29). The differences in habitual PA levels between the groups of older adults were reflected in walking performance and maximal aerobic capacity, confirm previous evidence $(6,20)$ and support the notion that at advanced age, increasing PA by exercise training can help to preserve physical function and performance.

We could not detect a statistical difference in muscle volume between young and older adults with similar levels of PA, indicating that habitual PA may be an important factor in the age-associated loss in muscle volume that has been observed previously (30). In our study, however, training status did not affect muscle volume in older adults, although this may be explained by the fact that the cohort consisted primarily of endurance-trained athletes and endurance training is less likely to improve lean mass compared to strength training (31). Despite the lack of difference in muscle volume, muscle strength was lower in older participants and differences in muscle strength corresponded to habitual PA stratification applied to the three older adults groups, which is in agreement with previous comparisons between active and sedentary older adults $(6,32)$.

Exercise efficiency refers to the energetic cost of physical activities such as walking and decreases in exercise efficiency likely contribute to the age-related inefficiency of locomotion and daily activities (21). As expected, lower net exercise efficiency was observed in the older participants compared to young adults in the present study, underlining an increased energy expenditure during exercise in the presence of comparable resting metabolic rate. This observation is in line with the findings from Distefano et al. (6), showing a lower exercise efficiency in older adults when compared to young adults with comparable levels of PA ( 8,500 vs. $\sim 10,500$ steps per day, respectively).

The relative difference of $\sim 15 \%$ in exercise efficiency in the trained older adults compared to the older adults with normal PA are similar to two previous studies suggesting that exercise training interventions have the potential to reverse agerelated reductions by increasing exercise efficiency up to $\sim 30 \%$ in sedentary (33) and endurance-trained volunteers (34). These findings are further supported by a 
cross-sectional study observing a higher exercise efficiency in athletic adults when compared to sedentary older adults (20). Interestingly, mitochondrial dysfunction has been implicated as a key factor in the reduced exercise efficiency with age which indicates mitochondria as the target for intervention to reverse this source of debilitation in older adults $(20,21)$. We observed a strong positive correlation between maximal mitochondrial capacity and gross exercise efficiency suggesting that mitochondrial capacity is indeed a determinant of exercise efficiency in aging.

Older adults responded in a less effective manner to the first perturbation when compared to the young participants, underlining the higher risk of falls in this population (7), even when PA is comparable. This observation confirms numerous other studies demonstrating age related differences in the ability to cope with such perturbations (for example: $(35,36)$ ). As PA was comparable between young and older adults in this study, it might be inferred that an increased fall risk with age cannot be entirely attributed to a decrease in PA, as is often suggested. During unperturbed walking, most spatiotemporal parameters and their variability were not affected by age, indicating that perhaps steady-state walking is more readily preserved with PA. Nevertheless, this only seems to be confirmed to a certain extent, as the trained older adults in the current study did not have significantly different unperturbed gait outcomes, compared to the normally active older adults.

One previous study reported that in a mixed group of older and younger adults, those who were recreational runners performed better on a forward falling task than the non-active participants (37). This pattern was not observed in the current study, as there were no clear differences between the TO and $\mathrm{O}$ groups on the perturbation tasks. It could be argued that this may be partly due to the fact that the $\mathrm{O}$ group actually had quite a high habitual PA but this did not seem to result in a lack of difference to the young adults. Another possibility is that the current gait perturbation task, which is more dynamic and in which it is practically impossible to predict and prepare for the perturbations, is simply more complex than the 'forward lean and release task' in such a way that high levels of PA do not benefit the performance of the task as much.

The ability to improve stability recovery following repeated gait perturbations was preserved in older adults with normal PA when compared to young adults. 
Furthermore, both the $\mathrm{O}$ and TO groups significantly reduced their required recovery steps by the ninth perturbation. These results confirm many previous reports of adaptability to gait perturbations being preserved in older age and reinforce the potential value of perturbation-based balance training in this population (38-41). However, it does appear that exercise training in older participants does not lead to a better ability to adapt to perturbations. A recent review paper (82) highlighted that reduced adaptability to perturbations is mainly seen in populations with disrupted sensory and nervous systems. As the $O$ and TO participants of the present study were in good health, adaptability may have already been sufficient in both groups, with little room for improvement through physical activity.

In the current study, we found that both in vivo $\mathrm{PCr}$ recovery rate constant and ex vivo mitochondrial respiration in skeletal muscle was lower in older compared to young adults. It is important to note that in the present study the decrease in mitochondrial function has been observed in older adults with PA levels being well above the general recommendations (42). These results suggest that maintaining a healthy level of PA is not sufficient to prevent an age-related decline in mitochondrial function. These findings are in line with the age-related decline in the mitochondrial ATP production rate (MAPR) observed in young and older adults who exercised less than 30 min a week $(14,43)$. An even more prominent age-related decline in MAPR was observed comparing young and older adults who were similarly endurancetrained (43), further hinting at an independent, aging-induced decline in skeletal muscle mitochondrial function. Nevertheless, findings of the present study are in contrast with previous observations by Distefano et al. $(6,22)$, who found no chronological age effect on mitochondrial function in deeply phenotyped young and older adults. This discrepancy could be explained by gender differences, as we included 9 male and 8 female volunteers in both groups while Distefano et al. included 8 male and 2 female participants in each group. Further research is needed to clearly define the effect of aging on skeletal mitochondrial function at different levels of PA and examine if that is different in male versus female volunteers (44).

Interestingly, ex vivo ADP-stimulated mitochondrial respiration in permeabilized muscle fibers was $\sim 15 \%$ lower in older adults when compared to young adults, whereas trained older adults displayed a $\sim 30 \%$ higher respiration in comparison to 
older adults with normal PA. These findings support the retainment of skeletal muscle plasticity in response to exercise with aging and indicate that mitochondrial respiratory function can be preserved through exercise $(6,43)$. In line with the present study, other reports indicate that exercise intensity is an important determinant of ameliorating age-related biological functions since rigorous exercise programs (such as high-intensity interval training) - but not modest aerobic activity was able to prevent age-related decline in mitochondrial respiration (45). In contrast with ex vivo assessed mitochondrial respiration results, in vivo mitochondrial function, assessed by the ${ }^{31} \mathrm{PC}$ recovery rate constant, was not found to be different between the older adult groups. This discrepancy could be explained by the fact that ${ }^{31} \mathrm{P}-\mathrm{MRS}$ may be confounded by limiting systemic factors such as adequate perfusion and oxygen delivery during and after exercise (46), and other characteristics of the microenvironment. Interestingly, mitochondrial density, estimated by OXPHOS protein levels, appeared to be similar between young and older participants, indicating that the age-related loss in mitochondrial function is not explained by a lower mitochondrial mass. In trained older adults, however, OXPHOS proteins were higher, suggesting that increased levels of mitochondrial content do underlie the positive effect of exercise training on mitochondrial function in older individuals. These results suggest that aging and exercise training affect mitochondrial function via distinct mechanisms. We did not observe a difference in either ex vivo or in vivo skeletal muscle mitochondrial capacity in physically impaired older individuals as compared to healthy older adults with normal PA. Due to the small sample size of this group, however, these outcomes should be interpreted with caution.

To further explore the relation between mitochondrial oxidative capacity and multiple aspects of muscle health, we subjected the entire cohort $(n=59)$ to correlative analyses. We found that besides ex vivo mitochondrial capacity also in vivo mitochondrial function associated with maximal aerobic capacity and exercise efficiency, which further supports that mitochondrial energetics are likely important factors in loss of muscle function with aging. Similar results have recently been found by Distefano et al. (6) and Gonzalez-Freire et al. (47). Moreover, mitochondrial function correlated with measures of gait variability, indicating that mitochondrial function potentially influences the efficiency and consistency of the gait pattern. 
Interestingly, we found that exercise efficiency correlated strongly with mitochondrial respiratory capacity, as well as with physical function parameters such as the 6MWT and the chair stand test. Taken together, our data support a role for mitochondrial capacity and in maintaining muscle function and indicate that exercise efficiency may be a good proxy for skeletal muscle mitochondrial capacity and muscle health.

The isolated impact of aging on skeletal muscle is difficult to unravel from the many other factors that change concurrently with aging, including decreased PA and increased adiposity (48). Despite similar levels of habitual of PA in the current study, body fat was higher and (whole-body) insulin sensitivity was lower in the older participants when compared to young. In comparison with older adults with normal PA, trained older adults displayed higher levels of whole-body insulin sensitivity, underscoring the ability of exercise training to improve insulin sensitivity and glucose uptake, also at an older age. However, since young and trained older adults also featured a lower adiposity, differences in body composition may (partly) mediate the observed differences in insulin sensitivity, independent of mitochondrial function. Increased adiposity in older participants has also been negatively associated with muscle function, independent of muscle mass (49). Furthermore, unhealthy aging is characterized by an augmented accumulation of intramyocellular lipids $(6,28)$, which could influence muscle function (6) and insulin sensitivity (50). Moreover, differences in BMI and body composition, inherent to the different study group characteristics, have been suggested to also influence skeletal muscle mitochondrial capacity $(22,51)$. Taken together, our data indicate that body fat accumulation with increasing age may be a contributing factor in potentiating the loss of muscle quality in older adults and suggest that high-intensity physical activity may be necessary to minimize changes in body composition over the lifespan and to mitigate muscle aging.

Our results suggest that, despite maintaining an adequate physical activity level, aging is associated with low in vivo and ex vivo mitochondrial capacity, maximal aerobic capacity, exercise efficiency, gait stability, muscle strength, insulin sensitivity, physical function, and increased body fat. Nevertheless, increasing physical activity through regular exercise training partially protects against these age-related declines in (mitochondrial) oxidative capacity and muscle health. Finally, we showed that mitochondrial capacity was positively associated with exercise 
efficiency and insulin sensitivity, supporting the idea that mitochondria represent a promising therapeutic target to negate the aging-associated deterioration of skeletal muscle health in order to preserve physical function and performance.

\section{METHODS}

\section{Participants}

Fifty-nine participants, including 17 young (9 male and 8 female) and 42 older individuals (23 male and 19 female), were recruited in the community of Maastricht and its surroundings through advertisements placed on the Maastricht University campus, in newspapers, supermarkets, and at sports clubs. The study was conducted in accordance with the principles of the declaration of Helsinki and approved by the Ethics Committee of the Maastricht University Medical Center+. All participants provided their written informed consent, and the study was registered at clinicaltrials.gov with identifier NCT03666013.

Prior to inclusion, all participants underwent a medical screening that included a medical questionnaire, a physical examination by a physician, and an assessment of physical function by means of the Short Physical Performance Battery, which includes a standing balance test, a 4-m walk test, and a chair-stand test. The SPPB score was calculated according to the cut-off points determined by Guralnik et al. (52). A sitting blood pressure measurement and an electrocardiogram (ECG) were also performed. After the screening procedure, participants were assigned to the following study groups: young individuals with normal physical activity (Y, 20-30 years), older adults with normal physical activity $(O, 65-80$ years), trained older adults (TO, 65-80 years) and physically impaired older adults (IO, 65-80 years). Participants were considered normally physically active if they completed no more than one structured exercise session per week, whereas participants were considered trained if they engaged in at least three structured exercise sessions of at least 1 hour each per week for an uninterrupted period of at least the past year. Participants were classified as older adults with impaired physical function (IO) in case of an SPPB score of $\leq 9$. Upon inclusion, further details on habitual physical activity levels were obtained during the study using accelerometry (ActivPAL).

Exclusion criteria were contra-indications for MRI examination, uncontrolled hypertension, the use of medication that could interfere with the results of the study, 
medical history of cardiovascular disease, type 2 diabetes mellitus, or other health problems that may hamper the safety of the individual during participation. Impairments in parameters of liver and kidney function were examined via determination of plasma aspartate aminotransferase (ASAT), alanine aminotransferase (ALAT), gamma-glutamyltransferase ( $\mathrm{G} T)$, bilirubin, and creatine.

\section{Experimental design}

This cross-sectional study was conducted at the Maastricht University Medical Centre+, The Netherlands, between September 2017 and March 2020.

The detailed assessment of muscle health consisted of various measurements divided over five study visits, equally distributed over a period of 5 weeks to allow for sufficient recovery between each visit and prevent interference between the various measurements (Supplementary Table 1). During their participation, participants were instructed to maintain their habitual diet and physical activity pattern, and in the three days preceding the test days, participants refrained from strenuous physical activity.

\section{Hyperinsulinemic-euglycemic clamp}

A hyperinsulinemic-euglycemic clamp was performed to assess peripheral insulin sensitivity, as previously described (53). Briefly, participants reported to the laboratory at 8 AM after an overnight fast from 10 PM. A fasted blood sample was taken and subsequently, a primed-continuous infusion of $d-[6,6-2 \mathrm{H} 2]-g l u c o s e$ $\left(0.04 \mathrm{ml} \mathrm{kg}^{-1} \mathrm{~min}^{-1}\right)$ was initiated (54). After 3 hours $(\mathrm{t}=180 \mathrm{~min})$, infusion of insulin $\left(40 \mathrm{mU} \mathrm{m} \mathrm{min}^{-1}\right.$ ) was started for a period of 2.5 hours. Based on the continuously monitored plasma glucose concentrations, the glucose infusion rate (GIR) was adjusted to maintain a steady-state plasma glucose level of approximately $5.0 \mathrm{mmol}$ $\mathrm{L}^{-1}$. Subsequently, whole-body insulin sensitivity was determined by calculating the M-value (mg kg-1 $\left.\mathrm{min}^{-1}\right)$. During the last 30 minutes of basal sampling $(\mathrm{t}=150-$ $180 \mathrm{~min}$ ) and during the last $30 \mathrm{~min}$ of the insulin infusion ( $\mathrm{t}=300-330 \mathrm{~min}$ ), blood samples were collected and indirect calorimetry (Omnical, IDEE, Maastricht, The Netherlands) was performed. Based on the measured oxygen and carbon dioxide concentrations, substrate oxidation rates were calculated using equations with the assumption that protein oxidation was negligible (55). Steele's single pool nonsteady state equations were used, allowing small differences in glucose concentrations to calculate glucose appearance (Ra) and (insulin-stimulated) glucose disposal (Rd) (54). The change in Rd (from baseline to clamp steady-state) 
was corrected for plasma insulin and glucose levels during the clamp ( $\left.\mathrm{S}_{\mathrm{i}}\right)$, as described in Remie et al. (53). Endogenous glucose production (EGP) was calculated as Ra minus exogenous glucose infusion rate.

Three individuals in the 10 group were excluded from participation in the clamp on the grounds of safety. As a result, the small number $(n=3)$ remaining made a comparison unviable and we therefore excluded the 10 group from the final insulin sensitivity comparisons.

\section{Maximal aerobic capacity}

Maximal aerobic capacity $\left(\dot{\mathrm{V}}_{2} \mathrm{max}\right)$ was assessed with concurrent ECG during a graded cycling test until exhaustion, as described previously (56). Briefly, after a warming-up period of 5 minutes at 50 Watt, the power output was increased every 2.5 minutes by 50 Watt until levels above $80 \%$ of the predicted maximal heart rate $(=$ 220 years - age) were observed. After this point, the test continued, and power output was increased every 2.5 minutes by 25 Watt until the participant could no longer pedal above 60 revolutions per minute. Consumed $\mathrm{O}_{2}$ and expired $\mathrm{CO}_{2}$ were measured continuously throughout the test using indirect calorimetry (Omnical, IDEE, Maastricht, The Netherlands) to determine $\dot{\mathrm{V}} \mathrm{O}_{2}$ max.

\section{Habitual physical activity}

Habitual physical activity was determined using an ActivPAL3 monitor (PAL Technologies, Glasgow, Scotland) for a consecutive period of 5 days, including two weekend days. The monitor was wrapped and attached to the skin on the anterior aspect of the upper leg using Tegaderm ( $\left.3 \mathrm{M}^{\mathrm{TM}}\right)$ in a waterproof fashion; non-wear was therefore not an issue. Data were uploaded using the software provided by ActivPAL and processed using customized software written in MATLAB R2013b (MathWorks, Natick, MA, USA). Besides the total amount of steps per day, the total stepping time was calculated in proportion to waking time per day. Stepping time (i.e. physical activity) was further classified into high-intensity physical activity (HPA; minutes with a step frequency $>110$ steps $\mathrm{min}^{-1}$ in proportion to waking time) and lower-intensity physical activity (LPA; minutes with a step frequency $\leq 110$ steps min1 in proportion to waking time) (42). Waking time was determined according to Van der Berg et al. (57). 


\section{Body composition}

Body composition (fat and fat-free mass) was determined at 8 AM after an overnight fast from $10 \mathrm{PM}$ the previous evening using air displacement plethysmography (BodPod®, COSMED, Inc., Rome, Italy).

\section{Muscle strength}

Muscle contractile performance was measured using the Biodex System 3 Pro dynamometer (Biodex® Medical Systems, Inc., Shirley, NY, USA). For the measurements, the participants were stabilized in the device with shoulder, leg, and abdominal straps to prevent compensatory movement. The test was performed with the left leg in all participants. To measure maximal muscle strength, each participant performed 30 consecutive knee extension and flexion movements (range of motion 120 degrees $\mathrm{s}^{-1}$ ). The peak torque of each extension and flexion was recorded and maximal isokinetic knee-extensor and knee-flexor torque was defined of the highest peak torque and corrected for fat-free mass $\left(\mathrm{Nm} \mathrm{kgFFM}^{-1}\right)$.

\section{Resting energy expenditure}

Resting energy expenditure (REE) and substrate utilization were measured at 8 AM, after an overnight fast from 10 PM the preceding evening. Gas exchange was measured by open-circuit respirometry with an automated ventilated hood system for 45 minutes. During the measurement, participants lay on a bed in a supine position. Data from the first 5 and last 5 minutes were omitted. REE data was adjusted for fatfree mass by calculating 'REE residuals', essentially according to Ravussin et al. (58).

\section{Submaximal exercise test and exercise energy expenditure}

After assessing resting energy expenditure, participants performed a 1-hour submaximal exercise bout in the fasted state on an electronically braked cycle ergometer. To reach equal levels of exercise intensity, submaximal cycle test was performed at $50 \%$ of $W_{\max }$ as measured during the maximal aerobic cycling test. Participants were instructed to pedal at a controlled cadence between 60 and 70 revolutions per minute. To calculate exercise energy expenditure (EEE) and substrate oxidation, $\mathrm{O}_{2}$ consumption and $\mathrm{CO}_{2}$ production were recorded using indirect calorimetry for 15 minutes at two time points ( $t=15 \mathrm{~min}$ and $\mathrm{t}=45 \mathrm{~min}$ ). The submaximal cycle test was performed at, on average, 109 (29) Watt versus 74 (23) Watt in young versus older adults, respectively $(p<0.001)$. The TO individuals 
performed the submaximal cycle test at higher absolute power (on average 97 (28) Watt) in comparison with $\mathrm{O}(74$ (23) Watt, $\mathrm{p}=0.030)$ and $\mathrm{IO}(60$ (26) Watt, $\mathrm{p}=$ $0.017)$.

\section{Calculations of energy expenditure and exercise efficiency}

Energy expenditure was measured in rest, upon insulin stimulation during the hyperinsulinemic-euglycemic clamp, and during the submaximal cycle test using indirect calorimetry. The Weir equation (59) was used to calculate whole-body energy expenditure from measurements of $\mathrm{O}_{2}$ consumption and $\mathrm{CO}_{2}$ production. Carbohydrate and fat oxidation rates were calculated using the non-protein equations by Péronnet and Massicotte (55).

During submaximal exercise test, gross energy efficiency (GEE) was computed as the power output (watts converted to $\mathrm{kJ} \mathrm{min}^{-1}$ ) over exercise energy expenditure (in $\mathrm{kJ} \mathrm{min}^{-1}$ ) during the 1-hour submaximal bike test and expressed as a ratio (Eq. 1) as described previously (60). Mean values of work, $\mathrm{VO}_{2}, \mathrm{VCO}_{2}$ were averaged over 15minute periods at two time points $(t=15 \mathrm{~min}$ and $t=45 \mathrm{~min})$. Data from the first 5 min and last 2 min were omitted.

$\operatorname{Eq~1:~} \operatorname{GEE}(\%)=\left(\frac{\text { Work }(\mathrm{kJ} \mathrm{min}-1)}{\operatorname{EEE}\left(\mathrm{kJ} \min ^{-1}\right)}\right) \cdot 100$

Net energy efficiency (NEE) was measured from the submaximal test as power output (watts converted to $\mathrm{kJ} \mathrm{min}^{-1}$ ) over EE during exercise (EEE) minus resting EE (REE) (Eq. 2) as described by (60). REE was measured on the same day as described above.

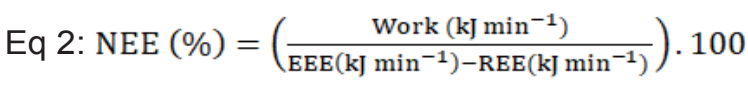

\section{Skeletal muscle biopsy}

Prior to the submaximal exercise test, a muscle biopsy was taken from the $m$. vastus lateralis under local anesthesia (1.0\% lidocaine without epinephrine) according to the Bergström method (61). Part of the biopsy was immediately placed in an ice-cold preservation medium (BIOPS, OROBOROS Instruments, Innsbruck, Austria) and used to measure ex vivo mitochondrial oxidative capacity. The remaining part of the 
muscle biopsy was immediately frozen in melting isopentane and stored at $-80^{\circ} \mathrm{C}$ until further analysis.

\section{Western blot analysis}

Mitochondrial content was assessed by mitochondrial OXPHOS protein expression using western blot analyses in Bioplex-lysates of human muscle tissue as previously described (62). Equal amounts of protein were loaded 4-12\% Bolt gradient gels (Novex, Thermo Fisher Scientific, Bleiswijk, The Netherlands). Proteins were transferred to nitrocellulose with the Trans-Blot Turbo transfer system (Bio-Rad Laboratories). Primary antibodies contained a cocktail of mouse monoclonal antibodies directed against human OXPHOS (dilution 1:10,000; ab110411, Abcam, Cambridge, UK). The hOxPHOS proteins were detected using secondary antibodies conjugated with IRDye680 or IRDye800 and were quantified with the CLx Odyssey Near-Infrared Imager (Li-COR, Westburg, Leusden, The Netherlands).

\section{Ex vivo high-resolution respirometry}

Permeabilized skeletal muscle fibers were immediately prepared from the muscle tissue collected in the preservation medium, as described previously (63). Subsequently, the permeabilized muscle fibers ( $2.5 \mathrm{mg}$ wet weight) were analyzed for mitochondrial function using an oxygraph (OROBOROS Instruments, Innsbruck, Austria), according to Hoeks et al. (63). To prevent oxygen limitation, the respiration chambers were hyper-oxygenated up to $\sim 400 \mu \mathrm{mol} \mathrm{L}^{-1} \mathrm{O}_{2}$. Subsequently, two different multi-substrate/inhibition protocols were used in which substrates and inhibitors were added consecutively at saturating concentrations. State 2 respiration was measured after the addition of malate $\left(4 \mathrm{mmol} \mathrm{L}^{-1}\right)$ plus octanoyl-carnitine (50 $\left.\mu \mathrm{mol} \mathrm{L} \mathrm{L}^{-1}\right)$ or malate $\left(4 \mathrm{mmol} \mathrm{L}^{-1}\right)$ plus glutamate $\left(10 \mathrm{mmol} \mathrm{L}^{-1}\right)$. Subsequently, an excess of $2 \mathrm{mmol} \mathrm{L}^{-1}$ of ADP was added to determine coupled (state 3 ) respiration. Coupled respiration was then maximized with convergent electron input through Complex I and Complex II by adding succinate $\left(10 \mathrm{mmol} \mathrm{L}^{-1}\right)$. Finally, the chemical uncoupler carbonylcyanide-4-(trifluoromethoxy)-phenylhydrazone (FCCP) was titrated to assess the maximal capacity of the electron transport chain (state $3 u$ respiration) or oligomycin $\left(2 \mu \mathrm{g} \mathrm{ml}^{-1}\right.$ ) was added to assess the respiration not coupled to ATP synthesis (state 40 respiration). The integrity of the outer mitochondrial membrane was assessed by the addition of cytochrome C (10 $\mu \mathrm{mol} \mathrm{L}^{-}$ $\left.{ }^{1}\right)$ upon maximal coupled respiration. If cytochrome $C$ increased oxygen consumption 
$>15 \%$, the measurement was excluded to assure the viability and quality of the muscle mitochondrial measurement. All measurements were performed in quadruplicate.

\section{Magnetic resonance spectroscopy (Muscle volume and PCr recovery)}

All magnetic resonance (MR) experiments were performed on the same day on a 3T whole body MRI scanner (Achieva 3T-X; Philips Healthcare, Best, The Netherlands). To standardize food intake, participants consumed a light lunch at noon and remained fasted until completion of all MR experiments. Participants reported to the university at 2:30 PM and were seated in the waiting room for at least 30 minutes to minimize the possible effect of prior muscle activity. At 3 PM, participants were positioned supine in the MR scanner to determine muscle volume with a series of T1-weighted images of the upper leg (slice thickness $=10.0 \mathrm{~mm}$, no gap between slices, in-plane resolution $=0.78 \times 0.78 \mathrm{~mm}$ ). A custom-written MATLAB 2016a script (The Mathworks Inc. Natick, MA, USA) was used to semi-automatically segment adipose tissue and muscle and quantify muscle volume. The muscle segmentation was performed in the consecutive slices between the lower end of the $m$. rectus femoris and the lower end of the $m$. gluteus maximus.

Subsequently, phosphorus magnetic resonance spectroscopy ( ${ }^{31} \mathrm{P}$-MRS) was performed to measure in vivo mitochondrial function in $m$. vastus lateralis as previously described (64), using a $6 \mathrm{~cm}$ surface coil. A series of 150 unlocalized ${ }^{31} \mathrm{P}$ spectra was acquired using the following parameters: single acquisitions (NSA $=1$ ); repetition time $(T R)=4000 \mathrm{~ms}$; spectral bandwidth $=3000 \mathrm{~Hz}$; number of points = 1024. The 150 spectra were divided into 10 spectra at rest, 70 spectra during kneeextension exercise, and 70 spectra during recovery. Exercise within the scanner was performed to an auditory cue $(0.5 \mathrm{~Hz})$ in a custom-built knee-extension device with adjustable weight. The intensity was chosen to correspond to $50-60 \%$ of the predetermined maximum weight (determined on a separate day). Spectra were analyzed with a custom-made MATLAB 2016a script. PCr, ATP, and inorganic phosphate peaks were fitted, and $\mathrm{pH}$ was determined. The $\mathrm{PCr}$ recovery was fitted with a mono-exponential function and the rate constant $\left(\kappa\right.$ in $\left.\mathrm{s}^{-1}\right)$ was determined as previously described (64). The rate constant $\kappa$ of $\mathrm{PCr}$ resynthesis is almost entirely dependent on ATP produced by oxidative phosphorylation and can therefore be used as a parameter of in vivo oxidative capacity (65). 


\section{Walking performance and unperturbed and perturbed walking stability}

To further determine physical function, walking performance and stability were quantitatively assessed during a self-paced 6MWT, during multiple fixed-speed gait trials, and during a repeated balance disturbance trial. All tasks were measured using a dual-belt, force plate-instrumented $(1000 \mathrm{~Hz})$ treadmill within a virtual environment (Computer Assisted Rehabilitation Environment Extended, CAREN; Motekforce Link, Amsterdam, The Netherlands) and a 12-camera motion capture system (100 Hz; Vicon Motion Systems, Oxford, UK).

The 6MWT began with an explanation and one familiarization session (including two "start-ups" to let participants become accustomed to the self-paced function) on the CAREN prior to completing the recorded 6MWT. The 6-minute walk distance was taken to represent performance (66). To assess gait variability, the means and coefficients of variation of step time, step length, step width, and double support time were measured during fixed-speed trials. This session again began with familiarization trials, followed by recorded trials (2-3 minutes - minimum of 120 steps) at speeds of $0.4-1.8 \mathrm{~m} \mathrm{~s}^{-1}$ in $0.2 \mathrm{~m} \mathrm{~s}^{-1}$ steps (67). These trials were then analyzed to calculate a stability-normalized walking speed for each participant individually for use in the gait perturbation trial, set for a margin of stability (MoS see below; (27)) of $0.05 \mathrm{~m}$ as described previously (68), ensuring a comparable baseline gait stability for all groups (69).

The perturbation trial included 3 to 4 minutes of unperturbed walking, followed by 10 unannounced unilateral treadmill belt acceleration perturbations (every 30 to 90 seconds) as reported previously (38). The first perturbations to each limb were analyzed (Pert1 $\mathrm{R}$ and Pert2L), representing novel disturbances, as well as the ninth perturbation (final left leg perturbation; Pert9L) to indicate adaptation in gait following eight repeated perturbations. The anteroposterior MoS at foot touchdown were calculated (27), adapted for our validated reduced kinematic model (70). The MoS was calculated for the following steps: the mean MoS of the eleventh to second last step before each perturbation (Base); the final step before each perturbation (Pre); and the first eight recovery steps following each perturbation (Post1-8).

The number of steps needed to return to pre-perturbation stability was determined by calculating the number of steps within $0.05 \mathrm{~m}$ of the MoS value of Base for each individual, counting back from the eighth recovery step, using custom written $\mathrm{R}$ code ( $R$ version 3.6.0). 
For all three gait tasks, initial data processing was conducted in MATLAB $2016 a$. The three-dimensional coordinates of the markers were filtered using a low pass second-order Butterworth filter (zero-phase) with a $12 \mathrm{~Hz}$ cut-off frequency. Foot touchdown and toe-off were detected using a combined method of force plate data (50 N threshold) and foot marker data, as described in detail previously (67). Only three individuals in the $1 \mathrm{O}$ group were able to complete the perturbation trial; as such the IO group was excluded from the perturbation analyses.

\section{Statistics}

Statistical analyses were performed using SPSS 26.0 (IBM, Chicago, IL, USA). Data are reported as mean (SD) unless stated otherwise. Analyses were performed for $n$ $=59$ unless specified otherwise in the table and the figure descriptions. The distribution of sex across groups was determined by $x^{2}$ test. In relation to the first research aim, differences between $Y$ and $O$ were tested by two-sided, independent samples t-test. To address the second research aim, group differences between $O$, TO, and 10 were determined by means of a one-way analysis of variance (ANOVA) with Tukey's post-hoc test or by a two-sided Kruskal-Wallis test with Bonferroni correction, as appropriate. Of note: the same group of older adults with normal physical activity $(\mathrm{O})$ was included in both comparisons.

GraphPad Prism version 8.02 for Windows was used for statistical analysis of the gait tasks (GraphPad Software Inc., La Jolla, California, USA). For the fixed-speed gait trials, mixed-effects models using the restricted maximum likelihood method with group ( $\mathrm{Y}$ and $\mathrm{O}$ or $\mathrm{O}$ and $\mathrm{TO}$ ) and walking speed (repeated measure: $0.4,0.6,0.8$, $1.0,1.2,1.4$, and $1.6 \mathrm{~m} \mathrm{~s}^{-1}$ ) as factors were conducted for the mean gait parameters and their variability. For the perturbation trials, two-way repeated measures ANOVAs with group ( $\mathrm{Y}$ and $\mathrm{O}$ or $\mathrm{O}$ and $\mathrm{TO}$ ) and step (repeated measures; Base, Pre, Post18) as factors were conducted for perturbations Pert $1 \mathrm{R}$, Pert $2 \mathrm{~L}$ and Pert9L with Dunnett's or Sidak's tests for multiple comparisons. Regarding the number of recovery steps required, Wilcoxon matched-pairs signed rank tests were used to test for differences between the perturbations with the groups and Mann-Whitney tests were used to compare the groups to each other.

To assess associations between variables, we conducted bivariate Pearson or Spearman correlation and partial correlation analyses corrected for sex, age, and BMI. For all tests $p<0.05$ was considered statistically significant. 


\section{Data availability}

Source data for all figures and tables have been provided with this paper (Supplementary Data). All other data supporting the findings of this study are available from the corresponding author upon reasonable request.

\section{Acknowledgements}

The project is organized by and executed under the auspices of TiFN, a publicprivate partnership on precompetitive research in food and nutrition. Funding for this research was obtained from Danone Nutricia Research, Friesland Campina, the Netherlands Organisation for Scientific Research, and the Top-sector Agri\&Food.

\section{Author contributions}

L.G. designed and performed the experiments, analyzed the data, and wrote the manuscript. N.J.C., C.M., C.E.F., L.B., Y.M.H.B., J.M., J.A.J., E.M-K., G.S., and B.H., assisted during the experiments, the data analysis, and reviewed and edited the manuscript. V.B.S., J.v.d.B., M.C.E.B., K.M., P.S., and J.H. contributed to the design of the study, interpretation of the data, and reviewed and edited the manuscript.

\section{Conflict of interest}

The authors declare no competing interests. Author M.C.E.B is affiliated with Friesland-Campina and author J.v.d.B is affiliated Danone Nutricia Research. Friesland-Campina and Danone Nutricia Research are sponsors of the TiFN program and partly financed the project. They had no role in data collection and analysis and decision to publish. Author M.C.E.B and J.v.d.B declare that they have no conflict of interest. 


\section{SUPPLEMENTARY MATERIAL FOR CHAPTER 3}

\section{Comparable gait variability during unperturbed walking in older adults}

Fourteen out of fifteen young participants completed all walking speed trials, with one person not able to walk at $1.8 \mathrm{~m} \mathrm{~s}^{-1}$ for two minutes. Twelve of seventeen old participants completed all speeds, with five unable to complete the $1.8 \mathrm{~m} \mathrm{~s}^{-1}$ speed. These differences between groups in the walking speeds generally align with the 6MWT results. When compared to young, older participants had a larger step length variability $\left(F_{(1,30)}=7.077, p=0.012\right.$; significant pairwise comparisons at $1.2,1.4$, and $1.6 \mathrm{~m} \mathrm{~s}^{-1}$; Supplementary Figure 1), but no other significant differences were found.

\section{Less gait stability but comparable adaptability during balance disturbances in older adults}

All participants were able to recover from the gait perturbations without harness assistance. When considering group differences for the entire perturbation trial, the first perturbation (Pert1 $R$ ) had a significantly larger effect on the margin of stability (MoS) in the older adults compared to the young participants (Pert1R: $F_{(1,28)}=7.7, p$ $=0.010)$. For the second (Pert2 $\mathrm{L})$ and ninth (Pert9 $\mathrm{L})$ perturbations, no significant group differences were observed (Pert2L: $F_{(1,29)}=0.6, p=0.435$; Pert9L: $F_{(1,29)}=$ $0.001, p=0.976)$, indicating that older adults initially perform poorer than young adults, but the ability of older adults to adapt gait in response to repeated perturbations is intact. Furthermore, significant step by age group interaction effects on MoS were found for Pert1 $\mathrm{R}$ and Pert2L but not Pert9L $\left(\right.$ Pert1 $_{\mathrm{R}}: \mathrm{F}_{(9,252)}=6.3, \mathrm{p}<$ 0.001; Pert2L: $F_{(9,261)}=3.539, p<0.001 ;$ Pert9L: $\left.F_{(9,261)}=1.413, p=0.182\right)$, indicative of different age-related recovery stepping behavior in the first two but not ninth perturbation. For all perturbations, the older adults' recovery steps, in comparison to the young adults, were more frequently different to Base and these differences also occurred in later recovery steps (significant results indicated by * in Supplementary Figure 2a).

When considering group differences at step level, the MoS in the first and second step after Pert1 $\mathrm{R}$ and in the first step after Pert2L were observed to be significantly different between young and old (significant results indicated by \# in Supplementary Figure 2a). These results indicate that the largest age group effect is seen in the first 1-2 recovery steps of the first two perturbations 
The young group required averages of 5.7 (1.8), 5.7 (1.6), and $3.6(2.1)$ recovery steps and the older group required averages of 6.2 (2.4), 6.2 (2.1), and 4.2 (2.1) recovery steps for Pert1R, Pert2L, and Pert9L, respectively (Supplementary Figure $2 b)$. Both the young and older group required significantly fewer recovery steps during Pert9 $L$ than both Pert1R and Pert2L (Supplementary Figure $2 b$ ). No significant group differences were observed in the number of recovery steps for each perturbation.

\section{Comparable gait variability during unperturbed walking in trained older adults}

In O, twelve out of seventeen participants completed all speeds, with five participants unable to complete the $1.8 \mathrm{~m} \mathrm{~s}^{-1}$ speed. Of TO, all eighteen participants could complete all speeds. For IO, one participant completed all speeds, one completed all except $1.8 \mathrm{~m} \mathrm{~s}^{-1}$, two reached $1.4 \mathrm{~m} \mathrm{~s}^{-1}$, and one reached $1.2 \mathrm{~m} \mathrm{~s}^{-1}$. These differences between groups in the walking speeds successfully achieved generally align with the 6MWT results. When comparing $\mathrm{O}$ and TO, no significant differences were observed for gait variability, including step time, step length, step width, and double support (Supplementary Figure 3).

A second set of similar analyses were conducted for the speeds $0.4 \mathrm{~m} \mathrm{~s}^{-1}$ up to 1.2 $\mathrm{m} \mathrm{s}^{-1}$ including the $\mathrm{O}$, TO, and $\mathrm{IO}$ groups. Here, a significant effect of group was found for double support time variability $\left(F_{(2,37)}=4.045, p=0.0258\right)$, with the $1 O$ group showing (non-significantly) more variability than the other two groups. No other group effects or pairwise comparisons were significant. Data shown in Supplementary Figure 4 also indicate non-significant increases in step width, step time variability, and (to a lesser extent) step length variability in IO. Together, the results indicate a less stable gait in 10 , despite the insignificant findings due to the small sample size.

\section{Comparable gait stability and adaptability in trained older adults}

During the gait stability assessments, all measured participants were able to recover from the gait perturbations without harness assistance. When considering the entire perturbation trial (Supplementary Figure $5 a$ ), MoS was not significantly different between $O$ and TO for Pert1R, Pert2L, and Pert9L (Pert1R: $F_{(1,32)}=0,066, p=0.8$; Pert2L: $F_{(1,32)}=0.019, p=0.892$; Pert9L: $\left.F_{(1,33)}=0.95, p=0.338\right)$. Furthermore, no significant step by group interaction effects on MoS were found for Pert1R, Pert2L, or 
Pert9L (Pert1R: $F_{(9,288)}=0.62, p=0.784 ;$ Pert2L: $F_{(9,288)}=0.88, p=0.544 ;$ Pert9L: $F_{(9,}$ $297)=1.64, p=0.102)$. These results indicate no significant stability or stepping behavior difference between $\mathrm{O}$ and TO. No significant differences in MoS between the groups at step level were observed (Supplementary Figure 5a).

Following Pert1R, Pert2L, and Pert9L, O required 6.2 (2.4), 6.2 (2.1), and 4.2 (2.1) recovery steps, respectively (Figure 13b). Following the same perturbations, TO required $6.1(2.2), \quad 5.2(2.1)$, and 4.4 (2.5) recovery steps, respectively (Supplementary Figure $5 \mathrm{~b}$ ). O participants required significantly fewer recovery steps during Pert9 $\mathrm{L}$ than both Pert1 $\mathrm{R}$ and Pert2L, while the TO adults required significantly fewer recovery steps during Pert9 $\mathrm{L}_{\mathrm{L}}$ than Pert $1_{\mathrm{R}}$ (Supplementary Figure $5 \mathrm{~b}$ ). No significant group differences on number of recovery steps for each perturbation were revealed. Three participants of the $\mathrm{IO}$ group started and completed the perturbation trial, while three 10 participants were unable to walk fast enough to maintain the stability-normalized walking speed. Therefore, they were not included in the previous analyses. Following Pert1R, Pert2L, and Pert9L, IO required 7.6, 6.0, and 4.6 recovery steps, respectively, with all three 10 participants reducing their number of required recovery steps. 


\section{Supplementary Figures}
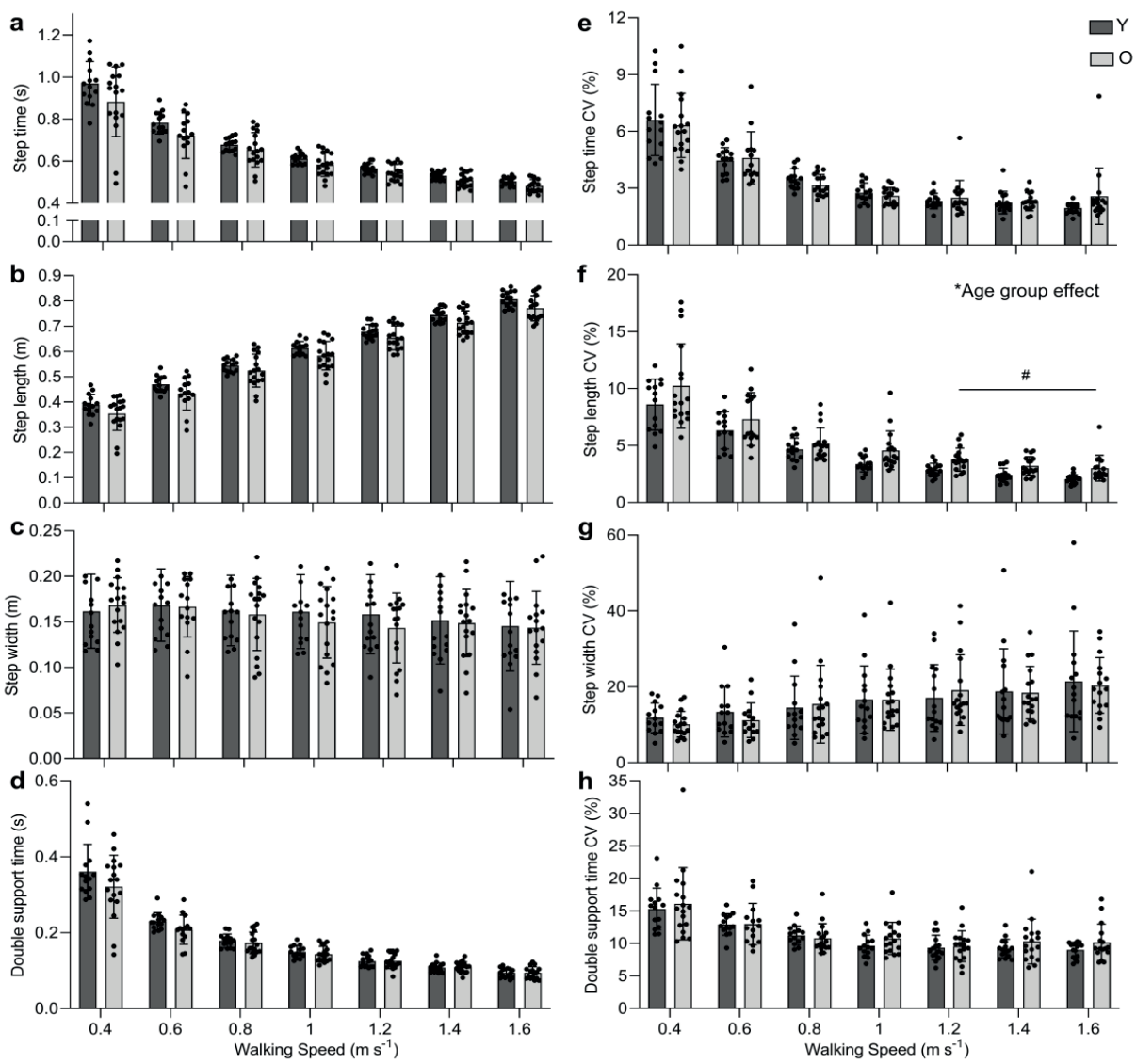

\section{Supplementary Figure 1}

Effect of aging on gait variability at different walking speeds $\left(0.4-1.6 \mathrm{~m} \mathrm{~s}^{-1}\right)$. a-d Absolute values for step time, step length, step with, and double support time. e-h Coefficients of variation (CV) of step time, step length, step width, and double support time. Dark grey bars represent the young individuals $(Y, n=14)$; light grey bars represent older individuals $(O, n=$ 17). One $Y$ participant was excluded due to issues in data collection and two $Y$ participants were not measured (one due to technical issues and one due to SARS-CoV-19 restrictions). Mixed-effects models using the restricted maximum likelihood method with age group and walking speed (repeated measure) as factors were conducted with pairwise Šídák's multiple comparisons test between groups at each speed. Values are presented as mean $\pm S D$ (with individual data points), * denotes age group effect denotes significant age group effect ( $p<$ $0.05)$ and \# with line indicates pairwise differences between the two groups $(p<0.05)$. 

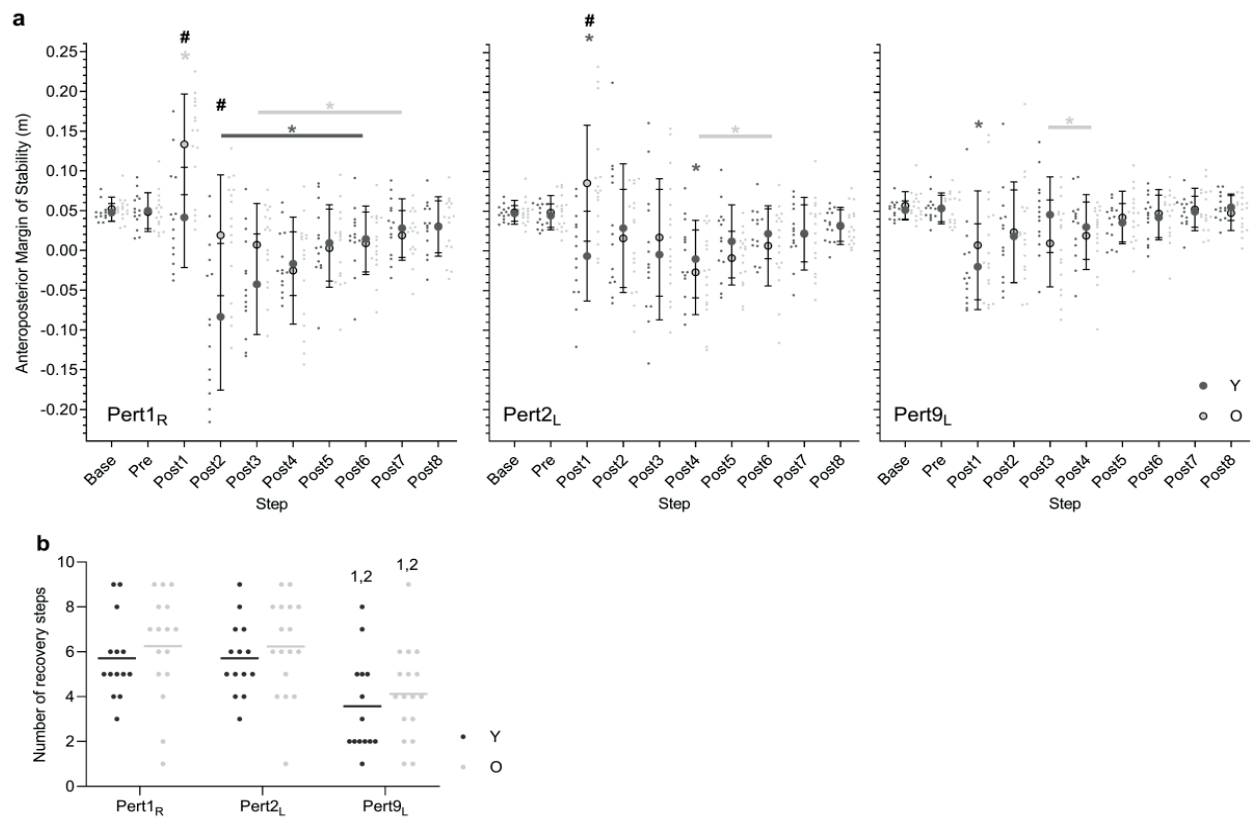

\section{Supplementary Figure 2}

Effect of aging on gait stability. a Mean and SD (with individual data points) of the anteroposterior margins of stability during the first, second, and ninth perturbations (Pert $1_{R}$, Pert2L, and Pert9 $\mathrm{L}$, respectively), including unperturbed walking prior to each perturbation (Base), the final step prior to each perturbation (Pre) and the first eight recovery steps following the perturbations (Post1 - 8) for young and older adults. Two-way repeated measures ANOVAs with group and step (repeated measures) as factors with within group pairwise comparisons to Base and between group pairwise comparisons were conducted. Dark grey* and light grey* indicate significant difference to Base for the young $(\mathrm{Y}$, dark grey dots, $n=12)$ and older (O, light grey dots, $n=13)$ groups, respectively $(p<0.05$, adjusted using Dunnett's multiple comparisons test). \# indicates significant difference between the young and older groups ( $p<0.05$; adjusted using Šídák's multiple comparisons test). $\mathbf{b}$ The number of recovery steps (means and individual values) required by the young (dark grey dots, $n=12$ ) and older adults (light grey dots, $n=13$ ) for the first, second, and ninth perturbations (Pert $1_{R}$, Pert $2_{L}$, and Pert9 ${ }_{L}$, respectively). ${ }^{1,2}$ indicate significant within-group difference to Pert $1_{R}$ and Pert2L, respectively $(p<0.05$, Wilcoxon matched-pairs signed rank tests). One $Y$ participant was excluded due to an issue in data collection and two $Y$ participants were not measured (one due to technical issues and one due to SARS-CoV-19 restrictions). Due to a technical issue with the treadmill, the data from the first perturbation of one of the $\mathrm{O}$ adults were excluded from the analysis. 

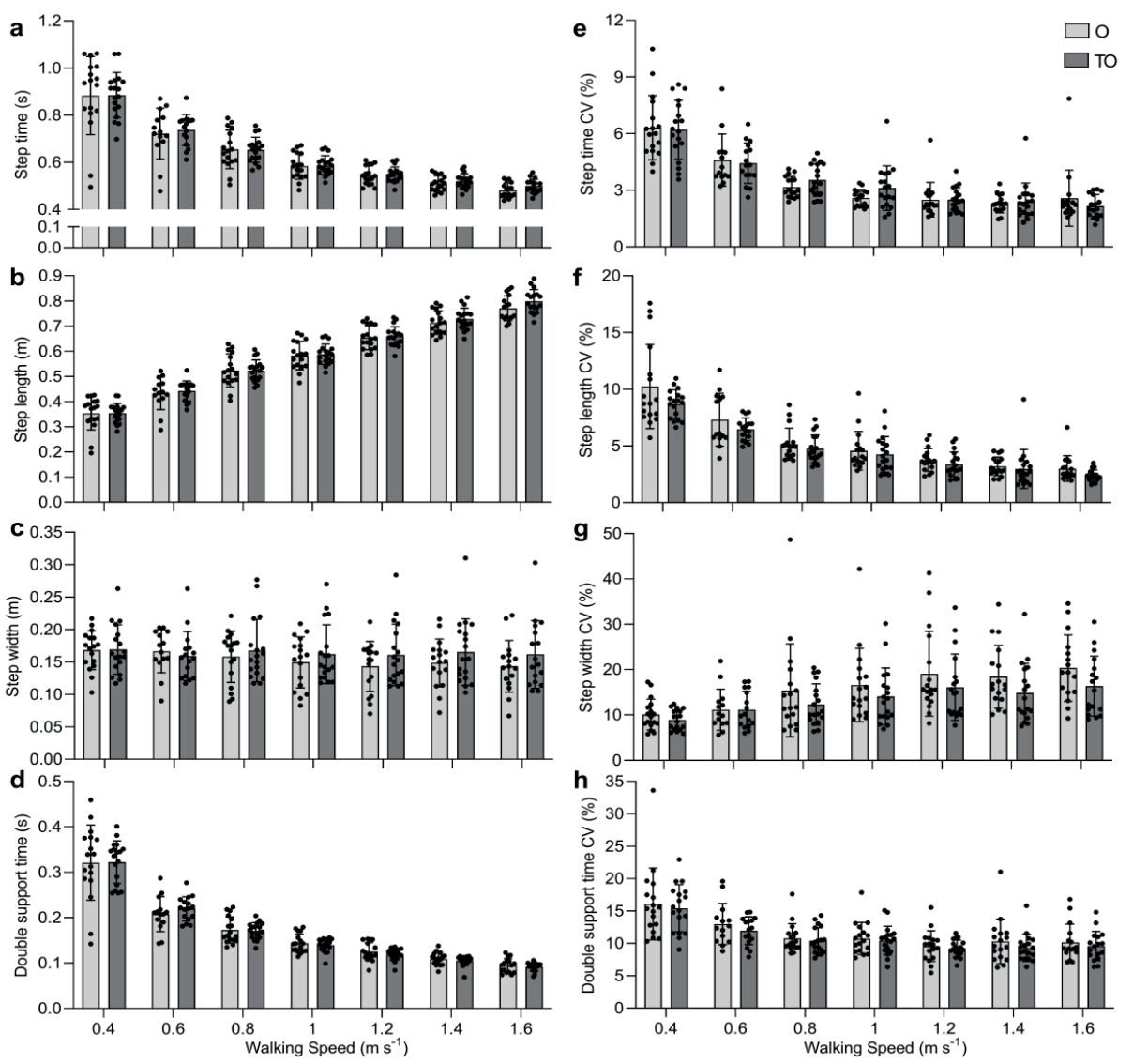

\section{Supplementary Figure 3}

Effect of exercise training on gait variability at different walking speeds $\left(0.4-1.6 \mathrm{~m} \mathrm{~s}^{-1}\right)$. a-d Absolute values for step time, step length, step with and, double support time. e-h Coefficients of variation (CV) of step time, step length, step width, and double support time. Light grey bars represent normally active older adults $(O, n=17)$; dark grey bars represent trained older adults (TO, $n=18)$. One TO participant was not measured due to scheduling difficulties. Mixed-effects models using the restricted maximum likelihood method with group and walking speed (repeated measure) as factors were conducted with pairwise Šídák's multiple comparisons test between groups at each speed. Values are presented as mean \pm SD (with individual data points). 

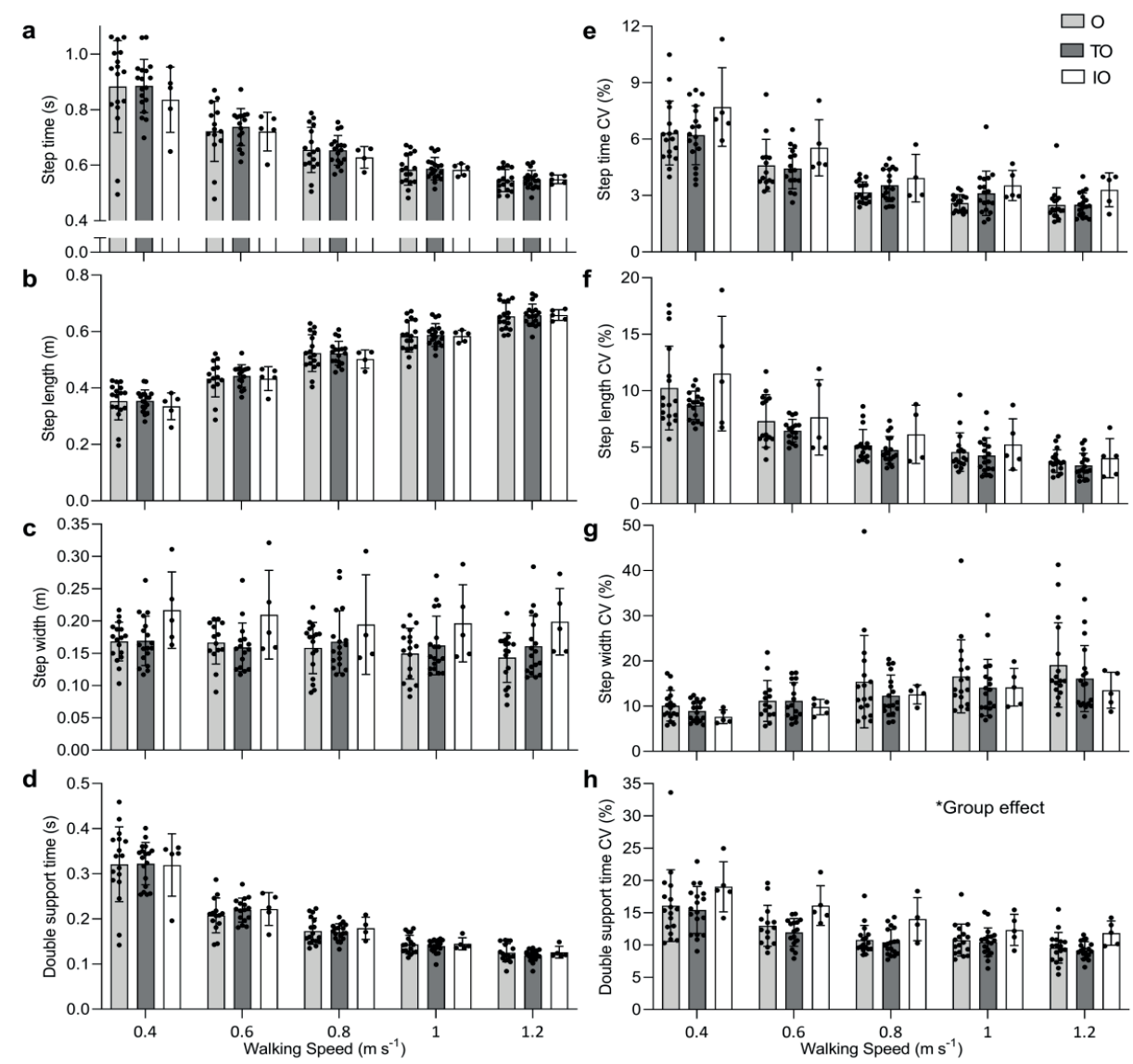

\section{Supplementary Figure 4}

Effect of exercise training and physical impairment on gait variability at different walking speeds $\left(0.4-1.2 \mathrm{~m} \mathrm{~s}^{-1}\right)$. a-d Absolute values for step time, step length, step width, and double support time. e-h Coefficients of variation (CV) of step time, step length, step width, and double support time. Light grey bars represent normally active older adults $(O, n=17)$; dark grey bars represent trained older adults $(\mathrm{TO}, \mathrm{n}=18)$; white bars represent physically impaired older adults $(\mathrm{IO}, \mathrm{n}=5)$. One $\mathrm{TO}$ and one $\mathrm{IO}$ participant were not measured due to scheduling difficulties. Mixed-effects models using the restricted maximum likelihood method with group and walking speed (repeated measure) as factors were conducted with pairwise Šídák's multiple comparisons test between groups at each speed. Values are presented as mean \pm SD (with individual data points), * denotes significant group effect $(p<0.05)$. 

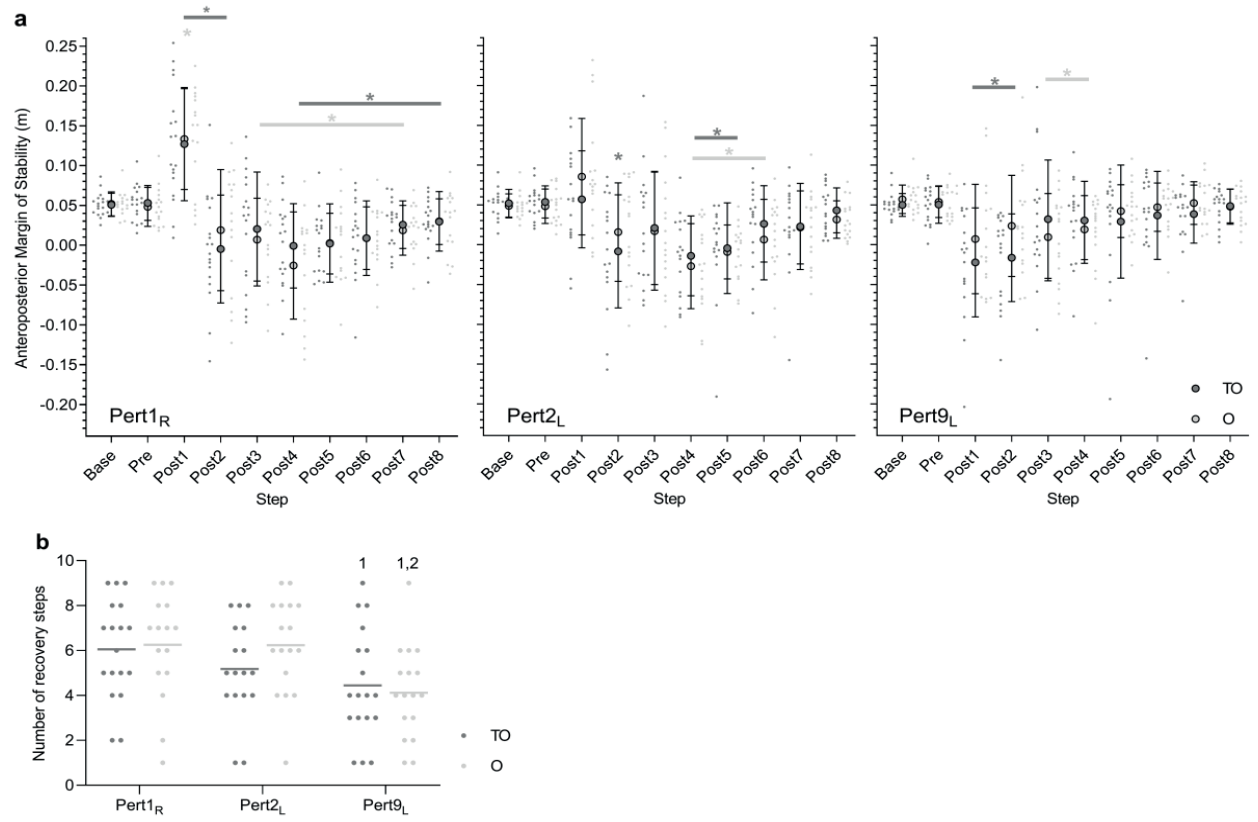

\section{Supplementary Figure 5}

Effect of exercise training on gait stability. a Means and \pm SD (with individual data points) of the anteroposterior margins of stability during the first, second, and ninth perturbations (Pert $1_{R}$, Pert $2_{L}$, and Pert9 $\mathrm{L}$, respectively), including unperturbed walking prior to each perturbation (Base), the final step prior to each perturbation (Pre) and the first eight recovery steps following the perturbations (Post1 - 8) for normally active older and trained older adults. Two-way repeated measures ANOVAs with group and step (repeated measures) as factors with within group pairwise comparisons to Base and between group pairwise comparisons were conducted. Dark grey * and light grey * indicate significant difference to Base for the $O$ (light grey dots, $n=13$ ) and TO (dark grey, dots $n=15$ ) groups, respectively ( $p<0.05$, adjusted using Dunnett's multiple comparisons test). b The number of recovery steps (means and individual values) required by the normally active older (light grey dots, $n$ = 13) and trained older adults (dark grey, dots $n=15$ ) for the first, second, and ninth perturbations (Pert $1_{R}$, Pert $2_{L}$, and Pert $9_{L}$, respectively). 1,2 indicate significant within-group difference to Pert $1_{R}$ and Pert 2 , respectively $(p<0.05$, Wilcoxon matched-pairs signed rank tests). One TO participant was not measured due to scheduling difficulties. Due to a technical issue with the treadmill, the data from the first perturbation of one of the $O$ participants and from the second perturbation of one of the TO participants were excluded from the analyses. Only three individuals in the $I O$ group were able to complete the perturbation trial, as such we compared only $\mathrm{O}$ versus TO. 


\section{Supplementary Tables}

Supplementary Table 1 Study outline.

\begin{tabular}{|c|c|c|c|c|c|}
\hline Screening & $\begin{array}{l}\text { Visit } 1 \\
\text { Week } 1\end{array}$ & $\begin{array}{l}\text { Visit } 2 \\
\text { Week } 2\end{array}$ & $\begin{array}{l}\text { Visit } 3 \\
\text { Week } 3\end{array}$ & \begin{tabular}{|l|} 
Visit 4 \\
Week 4
\end{tabular} & $\begin{array}{l}\text { Visit } 5 \\
\text { Week } 5\end{array}$ \\
\hline Medical questionnaire & $\begin{array}{l}\text { 1-step hyperinsulinemic } \\
\text { euglycemic }\end{array}$ & 6MWT (Caren) & $\begin{array}{l}\text { Body-composition } \\
\text { (bodpod) }\end{array}$ & Resting metabolic rate & Muscle volume (MRI) \\
\hline $\begin{array}{l}\text { Physical function } \\
\text { (SPPB) }\end{array}$ & & $\begin{array}{l}\mathrm{VO}_{2} \text { max cycle test } \\
{ }^{31} \mathrm{P}-\mathrm{MRS} \text { pre-test }\end{array}$ & $\begin{array}{l}\text { Gait variability and } \\
\text { dynamic balance test } \\
\text { (Caren) } \\
\text { Muscle strength } \\
\text { (Biodex) }\end{array}$ & \begin{tabular}{|l} 
Muscle biopsy \\
Ex vivo respirometry
\end{tabular} & $\begin{array}{l}\text { In vivo } \mathrm{PCr} \text { recovery } \\
\left({ }^{31} \mathrm{P}-\mathrm{MRS}\right)\end{array}$ \\
\hline & & & & Submaximal cycle test & \\
\hline \multicolumn{6}{|c|}{$\begin{array}{l}\text { Visit \#1 consisted of a 1-step hyperinsulinemic euglycemic clamp. On visit \#2, participants completed a } 6 \text {-minute walk test (6MWT) and } \\
\mathrm{VO}_{2} \text { max measurement. Furthermore, an ActivPAL accelerometer was provided to determine free-living physical activity over a period of } 5 \text { days } \\
\text { On visit \#3, participants completed a body composition measurement, a dynamic balance test, and quadriceps strength tests. On study visit \#4 } \\
\text { fasting blood samples were drawn, and resting energy expenditure was determined by indirect calorimetry followed by the muscle biops } \\
\text { procedure. Participants then completed the submaximal exercise test on a cycle ergometer. On visit \#5, participants completed the magneti } \\
\text { resonance imaging (MRI)/magnetic resonance spectroscopy (MRS) }\end{array}$} \\
\hline
\end{tabular}


Supplementary Table 2 Bivariate analysis to explore the relationships between energetics and physical muscle function.

\begin{tabular}{|c|c|c|c|c|c|c|c|c|c|c|c|c|c|c|}
\hline Measurement & Variables & & $\begin{array}{l}\text { 6MWT } \\
(\mathrm{m})\end{array}$ & $\begin{array}{l}\text { †Chair- } \\
\text { stand } \\
\text { test (s) }\end{array}$ & $\begin{array}{l}\mathrm{VO}_{2} \max \\
\left(\mathrm{ml} \mathrm{min}^{-1}\right. \\
\left.\mathrm{kgFFM}^{-1}\right)\end{array}$ & $\begin{array}{l}\text { Isokinetic } \\
\text { extension } \\
(\mathrm{Nm} \\
\left.\mathrm{kgFFM}^{-1}\right)\end{array}$ & $\begin{array}{l}\text { Isokinetic } \\
\text { flexion } \\
(\mathrm{Nm} \mathrm{kg} \\
\left.\mathrm{FFM}^{-1}\right)\end{array}$ & $\begin{array}{l}\text { M-value } \\
(\mu \mathrm{mol} \mathrm{kg} \\
\left.{ }^{1} \mathrm{~min}^{-1}\right)\end{array}$ & $\begin{array}{l}\mathrm{S}_{\mathrm{i}} \\
(\mu \mathrm{mol} \\
\mathrm{kgFFM}^{-1} \\
\left.\mathrm{~min}^{-1}\right)\end{array}$ & $\begin{array}{l}\text { Daily } \\
\text { steps } \\
\text { (counts } \\
\text { day }^{-1} \text { ) }\end{array}$ & $\begin{array}{l}\text { \#Recover } \\
\text { y steps } \\
\text { Pert1 }\end{array}$ & $\begin{array}{l}\text { Step } \\
\text { length } \\
\text { variability }\end{array}$ & $\begin{array}{l}\text { Double } \\
\text { support } \\
\text { time step } \\
\text { variability }\end{array}$ & $\begin{array}{l}\text { Gross } \\
\text { efficiency } \\
(\%)\end{array}$ \\
\hline \multirow[t]{6}{*}{$\begin{array}{l}\text { Mitochondrial } \\
\text { function }\end{array}$} & $\begin{array}{l}\text { Maximal coupled } \\
\text { respiration }\end{array}$ & $r$ & $0.335^{*}$ & -0.111 & $0.436^{*}$ & 0.080 & 0.126 & $0.379^{*}$ & 0.174 & $0.326^{*}$ & 0.105 & -0.177 & -0.258 & $0.345^{*}$ \\
\hline & $\begin{array}{l}\text { MOGS3 } \\
\left(\mathrm{pmol} \mathrm{s}^{-1} \mathrm{mg}^{-1}\right)\end{array}$ & $\mathrm{p}$ & 0.015 & 0.415 & 0.002 & 0.558 & 0.355 & 0.006 & 0.221 & 0.014 & 0.473 & 0.209 & 0.065 & 0.011 \\
\hline & $\begin{array}{l}\text { Maximal } \\
\text { uncoupled }\end{array}$ & r & $0.310^{*}$ & -0.146 & $0.512^{*}$ & 0.102 & 0.150 & $0.407^{*}$ & 0.232 & $0.372^{*}$ & 0.035 & -0.228 & $-0.324^{*}$ & $0.402^{*}$ \\
\hline & $\begin{array}{l}\text { respiration } \\
\left(\mathrm{pmol} \mathrm{s}^{-1} \mathrm{mg}^{-1}\right)\end{array}$ & $p$ & 0.025 & 0.282 & $<0.001$ & 0.454 & 0.269 & 0.003 & 0.101 & 0.005 & 0.812 & 0.103 & 0.019 & 0.003 \\
\hline & $\begin{array}{l}\mathrm{PCr} \text { recovery rate } \\
\text { constant } \mathrm{k}\left(\mathrm{s}^{-1}\right)\end{array}$ & $r$ & 0.240 & -0.075 & $0.482^{*}$ & 0.178 & 0.105 & 0.228 & 0.144 & 0.258 & -0.124 & $-0.283^{*}$ & $-0.313^{*}$ & $0.315^{*}$ \\
\hline & & $\mathrm{p}$ & 0.090 & 0.587 & 0.001 & 0.193 & 0.444 & 0.108 & 0.318 & 0.057 & 0.402 & 0.044 & 0.025 & 0.021 \\
\hline \multirow[t]{4}{*}{$\begin{array}{l}\text { Exercise } \\
\text { efficiency }\end{array}$} & $\begin{array}{l}\text { Gross efficiency } \\
(\%)\end{array}$ & $r$ & $0.679^{*}$ & $-0.485^{\star}$ & $0.711^{*}$ & $0.349^{*}$ & $0.356^{*}$ & $0.495^{*}$ & $0.337^{*}$ & $0.268^{*}$ & $-0.368^{*}$ & $-0.277^{*}$ & -0.193 & \\
\hline & & $\mathrm{p}$ & $<0.001$ & $<0.001$ & $<0.001$ & 0.008 & 0.007 & $<0.001$ & 0.015 & 0.046 & 0.008 & 0.044 & 0.166 & \\
\hline & $\begin{array}{l}\text { Net efficiency } \\
(\%)\end{array}$ & $r$ & $0.596^{*}$ & $0.454^{*}$ & $0.623^{*}$ & $0.276^{*}$ & 0.261 & $0.480^{*}$ & $0.356^{*}$ & 0.207 & $-0.349^{*}$ & -0.253 & -0.249 & \\
\hline & & $\mathrm{p}$ & $<0.001$ & $<0.001$ & $<0.001$ & 0.039 & 0.052 & $<0.001$ & 0.009 & 0.127 & 0.013 & 0.068 & 0.072 & \\
\hline
\end{tabular}

Data are from all groups (total $n>51$ ). * indicates the correlation is significant at the 0.05 level (two-sided, $p<0.05$ ); ${ }^{\dagger}$ indicates variables that were log transformed. MOGS3 = state 3 respiration upon malate + octanoyl-carnitine + glutamate + succinate; $6 \mathrm{MWT}=6$-minute walk test; $\mathrm{VO}_{2}$ max = maximal oxygen flow; FFM, = fat-free mass; $\mathrm{Nm}$ = newton meters; Pert1 = first perturbation during the balance challenge; $\mathrm{M}$-value, = mean glucose infusion rate; $S_{i}=$ insulin-stimulated glucose uptake; $r=$ pearson or \#spearman correlation coefficient as appropriate 


\section{REFERENCES}

1.Beard, J. R. et al. The World report on ageing and health: a policy framework for healthy ageing. The Lancet 387, 2145-2154 (2016).

2. Rodrigues, R., Huber, M. \& Lamura, G. Facts and Figures on Healthy Ageing and Longterm Care. Europe and North America, Occasional Reports Series 8. Vienna: European Centre (2012).

3. Roubenoff, R. Sarcopenia and its implications for the elderly. Eur J Clin Nutr 54, S40-S47 (2000).

4. Janssen, I., Heymsfield, S. B. \& Ross, R. Low Relative Skeletal Muscle Mass (Sarcopenia) in Older Persons Is Associated with Functional Impairment and Physical Disability. Journal of the American Geriatrics Society 50, 889-896 (2002).

5. Choi, S. et al. ${ }^{31} \mathrm{P}$ Magnetic Resonance Spectroscopy Assessment of Muscle Bioenergetics as a Predictor of Gait Speed in the Baltimore Longitudinal Study of Aging. GERONA 71, 1638-1645 (2016).

6. Distefano, G. et al. Physical activity unveils the relationship between mitochondrial energetics, muscle quality, and physical function in older adults: Mitochondria, muscle quality and physical function in aging. Journal of Cachexia, Sarcopenia and Muscle 9, 279-294 (2018).

7. Talbot, L. A., Musiol, R. J., Witham, E. K. \& Metter, E. J. Falls in young, middle-aged and older community dwelling adults: perceived cause, environmental factors and injury. BMC Public Health 5, 86 (2005).

8. Gadelha, A. B., Neri, S. G. R., Bottaro, M. \& Lima, R. M. The relationship between muscle quality and incidence of falls in older community-dwelling women: An 18-month follow-up study. Experimental Gerontology 110, 241-246 (2018).

9. Fried, L. P. et al. Frailty in Older Adults: Evidence for a Phenotype. The Journals of Gerontology Series A: Biological Sciences and Medical Sciences 56, M146-M157 (2001).

10. Crescenzo, R. et al. Skeletal Muscle Mitochondrial Energetic Efficiency and Aging. IJMS 16, 10674-10685 (2015).

11. dos Santos, L., Cyrino, E. S., Antunes, M., Santos, D. A. \& Sardinha, L. B. Sarcopenia and physical independence in older adults: the independent and synergic role of muscle mass and muscle function: Sarcopenia and physical independence in older adults. Journal of Cachexia, Sarcopenia and Muscle 8, 245-250 (2017).

12. Terroso, M., Rosa, N., Torres Marques, A. \& Simoes, R. Physical consequences of falls in the elderly: a literature review from 1995 to 2010. Eur Rev Aging Phys Act 11, 51-59 (2014).

13. Gonzalez-Freire, M. et al. Reconsidering the Role of Mitochondria in Aging. GERONA 70, 1334-1342 (2015).

14. Short, K. R. et al. Decline in skeletal muscle mitochondrial function with aging in humans. Proceedings of the National Academy of Sciences 102, 5618-5623 (2005).

15. Joseph, A.-M. et al. The impact of aging on mitochondrial function and biogenesis pathways in skeletal muscle of sedentary high- and low-functioning elderly individuals: Mitochondrial function in elderly individuals. Aging Cell 11, 801-809 (2012).

16. Jang, Y. C. et al. Increased superoxide in vivo accelerates age-associated muscle atrophy through mitochondrial dysfunction and neuromuscular junction degeneration. FASEB J 24, 1376-1390 (2010). 
17. Wanagat, J., Cao, Z., Pathare, P. \& Aiken, J. M. Mitochondrial DNA deletion mutations colocalize with segmental electron transport system abnormalities, muscle fiber atrophy, fiber splitting, and oxidative damage in sarcopenia. The FASEB Journal 15, 322-332 (2001).

18. Coen, P. M. et al. Skeletal Muscle Mitochondrial Energetics Are Associated With Maximal Aerobic Capacity and Walking Speed in Older Adults. The Journals of Gerontology: Series A 68, 447-455 (2013).

19. Zane, A. C. et al. Muscle strength mediates the relationship between mitochondrial energetics and walking performance. Aging Cell 16, 461-468 (2017).

20. Broskey, N. T. et al. Exercise efficiency relates with mitochondrial content and function in older adults. Physiol Rep 3, e12418 (2015).

21. Conley, K. E., Jubrias, S. A., Cress, M. E. \& Esselman, P. Exercise efficiency is reduced by mitochondrial uncoupling in the elderly: Mitochondrial uncoupling impacts exercise efficiency. Experimental Physiology 98, 768-777 (2013).

22. Distefano, G. et al. Chronological Age Does not Influence Ex-vivo Mitochondrial Respiration and Quality Control in Skeletal Muscle. GERONA glw102 (2016). doi:10.1093/gerona/glw102

23. Larsen, S. et al. The influence of age and aerobic fitness: effects on mitochondrial respiration in skeletal muscle. Acta Physiol (Oxf) 205, 423-432 (2012).

24. Ringholm, S. et al. Bed rest reduces metabolic protein content and abolishes exerciseinduced mRNA responses in human skeletal muscle. American Journal of PhysiologyEndocrinology and Metabolism 301, E649-E658 (2011).

25. Geng, T. et al. PGC-1a plays a functional role in exercise-induced mitochondrial biogenesis and angiogenesis but not fiber-type transformation in mouse skeletal muscle. American Journal of Physiology-Cell Physiology 298, C572-C579 (2010).

26. Sousa, A. S. et al. Sitting time and associated factors among Portuguese older adults: results from Nutrition UP 65. Eur J Ageing 17, 321-330 (2020).

27. Hof, A. L., Gazendam, M. G. J. \& Sinke, W. E. The condition for dynamic stability. Journal of Biomechanics 38, 1-8 (2005).

28. St-Jean-Pelletier, F. et al. The impact of ageing, physical activity, and pre-frailty on skeletal muscle phenotype, mitochondrial content, and intramyocellular lipids in men: Muscle phenotype, mitochondrial mass, and lipid content in human ageing. Journal of Cachexia, Sarcopenia and Muscle 8, 213-228 (2017).

29. Tudor-Locke, C., Hatano, Y., Pangrazi, R. P. \& Kang, M. Revisiting 'How Many Steps Are Enough?': Medicine \& Science in Sports \& Exercise 40, S537-S543 (2008).

30. Di Francesco, V. et al. Relationships between leisure-time physical activity, obesity and disability in elderly men. Aging Clin Exp Res 17, 201-206 (2005).

31. Lai, C.-C., Tu, Y.-K., Wang, T.-G., Huang, Y.-T. \& Chien, K.-L. Effects of resistance training, endurance training and whole-body vibration on lean body mass, muscle strength and physical performance in older people: a systematic review and network meta-analysis. Age and Ageing 47, 367-373 (2018).

32. Goodpaster, B. H. et al. Effects of physical activity on strength and skeletal muscle fat infiltration in older adults: a randomized controlled trial. J Appl Physiol 105, 6 (2008).

33. Woo, J. S., Derleth, C., Stratton, J. R. \& Levy, W. C. The Influence of Age, Gender, and Training on Exercise Efficiency. Journal of the American College of Cardiology 47, 10491057 (2006). 
34. Wang, E. et al. Impact of maximal strength training on work efficiency and muscle fiber type in the elderly: Implications for physical function and fall prevention. Experimental Gerontology 91, 64-71 (2017).

35. Karamanidis, K., Arampatzis, A. \& Mademli, L. Age-related deficit in dynamic stability control after forward falls is affected by muscle strength and tendon stiffness. Journal of Electromyography and Kinesiology 18, 980-989 (2008).

36. König, M., Epro, G., Seeley, J., Potthast, W. \& Karamanidis, K. Retention and generalizability of balance recovery response adaptations from trip perturbations across the adult life span. Journal of Neurophysiology 122, 1884-1893 (2019).

37. Karamanidis, K. \& Arampatzis, A. Age-related degeneration in leg-extensor muscletendon units decreases recovery performance after a forward fall: compensation with running experience. Eur J Appl Physiol 99, 73-85 (2006).

38. McCrum, C., Karamanidis, K., Grevendonk, L., Zijlstra, W. \& Meijer, K. Older adults demonstrate interlimb transfer of reactive gait adaptations to repeated unpredictable gait perturbations. GeroScience 42, 39-49 (2020).

39. Bohm, S., Mademli, L., Mersmann, F. \& Arampatzis, A. Predictive and Reactive Locomotor Adaptability in Healthy Elderly: A Systematic Review and Meta-Analysis. Sports Med 45, 1759-1777 (2015).

40. Grabiner, M. D., Crenshaw, J. R., Hurt, C. P., Rosenblatt, N. J. \& Troy, K. L. Exercisebased fall prevention: can you be a bit more specific? Exerc Sport Sci Rev 42, 161-168 (2014).

41. Gerards, M. H. G., McCrum, C., Mansfield, A. \& Meijer, K. Perturbation-based balance training for falls reduction among older adults: Current evidence and implications for clinical practice: Perturbation training for older adults. Geriatr Gerontol Int 17, 2294-2303 (2017).

42. Tudor-Locke, C. et al. How many steps/day are enough? for adults. Int J Behav Nutr Phys Act 8, 79 (2011).

43. Lanza, I. R. et al. Endurance Exercise as a Countermeasure for Aging. Diabetes 57, 2933-2942 (2008).

44. Sonjak, V. et al. Reduced Mitochondrial Content, Elevated Reactive Oxygen Species, and Modulation by Denervation in Skeletal Muscle of Prefrail or Frail Elderly Women. The Journals of Gerontology: Series A 74, 1887-1895 (2019).

45. Robinson, M. M. et al. Enhanced Protein Translation Underlies Improved Metabolic and Physical Adaptations to Different Exercise Training Modes in Young and Old Humans. Cell Metabolism 25, 581-592 (2017).

46. Adelnia, F. et al. The Role of Muscle Perfusion in the Age-Associated Decline of Mitochondrial Function in Healthy Individuals. Front. Physiol. 10, 427 (2019).

47. Gonzalez-Freire, M. et al. Skeletal muscle ex vivo mitochondrial respiration parallels decline in vivo oxidative capacity, cardiorespiratory fitness, and muscle strength: The Baltimore Longitudinal Study of Aging. Aging Cell 17, e12725 (2018).

48. Distefano, G. \& Goodpaster, B. H. Effects of Exercise and Aging on Skeletal Muscle. Cold Spring Harb Perspect Med 8, a029785 (2018).

49. Koster, A. et al. Does the Amount of Fat Mass Predict Age-Related Loss of Lean Mass, Muscle Strength, and Muscle Quality in Older Adults? The Journals of Gerontology Series A: Biological Sciences and Medical Sciences 66A, 888-895 (2011). 
50. Amati, F. et al. Skeletal Muscle Triglycerides, Diacylglycerols, and Ceramides in Insulin Resistance: Another Paradox in Endurance-Trained Athletes? Diabetes 60, 2588-2597 (2011).

51. Bharadwaj, M. S. et al. Relationships between mitochondrial content and bioenergetics with obesity, body composition and fat distribution in healthy older adults. BMC Obes $\mathbf{2}$, 40 (2015).

52. Guralnik, J. M. et al. A short physical performance battery assessing lower extremity function: association with self-reported disability and prediction of mortality and nursing home admission. J Gerontol 49, M85-94 (1994).

53. Remie, C. M. E. et al. Metabolic responses to mild cold acclimation in type 2 diabetes patients. Nat Commun 12, 1516 (2021).

54. Steele, R. Influences of glucose loading and of injected insulin on hepatic glucose output. Ann. N. Y. Acad. Sci. 82, 420-430 (1959).

55. Péronnet, F. \& Massicotte, D. Table of nonprotein respiratory quotient: an update. Can J Sport Sci 16, 23-29 (1991).

56. Kuipers, H., Verstappen, F. T. J., Keizer, H. A., Geurten, P. \& van Kranenburg, G. Variability of Aerobic Performance in the Laboratory and Its Physiologic Correlates. Int $J$ Sports Med 06, 197-201 (2008).

57. van der Berg, J. D. et al. Identifying waking time in 24-h accelerometry data in adults using an automated algorithm. Journal of Sports Sciences 34, 1867-1873 (2016).

58. Ravussin, E. et al. A 2-Year Randomized Controlled Trial of Human Caloric Restriction: Feasibility and Effects on Predictors of Health Span and Longevity. GERONA 70, 10971104 (2015).

59. Weir, J. B. de V. New methods for calculating metabolic rate with special reference to protein metabolism. The Journal of Physiology 109, 1-9 (1949).

60. Matomäki, P., Linnamo, V. \& Kyröläinen, H. A Comparison of Methodological Approaches to Measuring Cycling Mechanical Efficiency. Sports Med - Open 5, 23 (2019).

61. Bergström, J., Hermansen, L., Hultman, E. \& Saltin, B. Diet, Muscle Glycogen and Physical Performance. Acta Physiologica Scandinavica 71, 140-150 (1967).

62. van Moorsel, D. et al. Demonstration of a day-night rhythm in human skeletal muscle oxidative capacity. Molecular Metabolism 5, 635-645 (2016).

63. Phielix, E. et al. Lower Intrinsic ADP-Stimulated Mitochondrial Respiration Underlies In Vivo Mitochondrial Dysfunction in Muscle of Male Type 2 Diabetic Patients. Diabetes 57, 2943-2949 (2008).

64. Schrauwen-Hinderling, V. B. et al. Impaired in vivo mitochondrial function but similar intramyocellular lipid content in patients with type 2 diabetes mellitus and BMI-matched control subjects. Diabetologia 50, 113-120 (2007).

65. Kemp, G. J. \& Radda, G. K. Quantitative interpretation of bioenergetic data from 31P and $1 \mathrm{H}$ magnetic resonance spectroscopic studies of skeletal muscle: an analytical review. Magn Reson Q 10, 43-63 (1994).

66. Liu, W.-Y. et al. Reproducibility and Validity of the 6-Minute Walk Test Using the Gait Real-Time Analysis Interactive Lab in Patients with COPD and Healthy Elderly. PLoS ONE 11, e0162444 (2016).

67. McCrum, C. et al. The walking speed-dependency of gait variability in bilateral vestibulopathy and its association with clinical tests of vestibular function. Sci Rep 9, 18392 (2019). 
68. McCrum, C., Willems, P., Karamanidis, K. \& Meijer, K. Stability-normalised walking speed: A new approach for human gait perturbation research. Journal of Biomechanics 87, 48-53 (2019).

69. McCrum, C., Karamanidis, K., Willems, P., Zijlstra, W. \& Meijer, K. Retention, savings and interlimb transfer of reactive gait adaptations in humans following unexpected perturbations. Commun Biol 1, 230 (2018).

70. Süptitz, F., Catalá, M. M., Brüggemann, G.-P. \& Karamanidis, K. Dynamic stability control during perturbed walking can be assessed by a reduced kinematic model across the adult female lifespan. Human Movement Science 32, 1404-1414 (2013). 


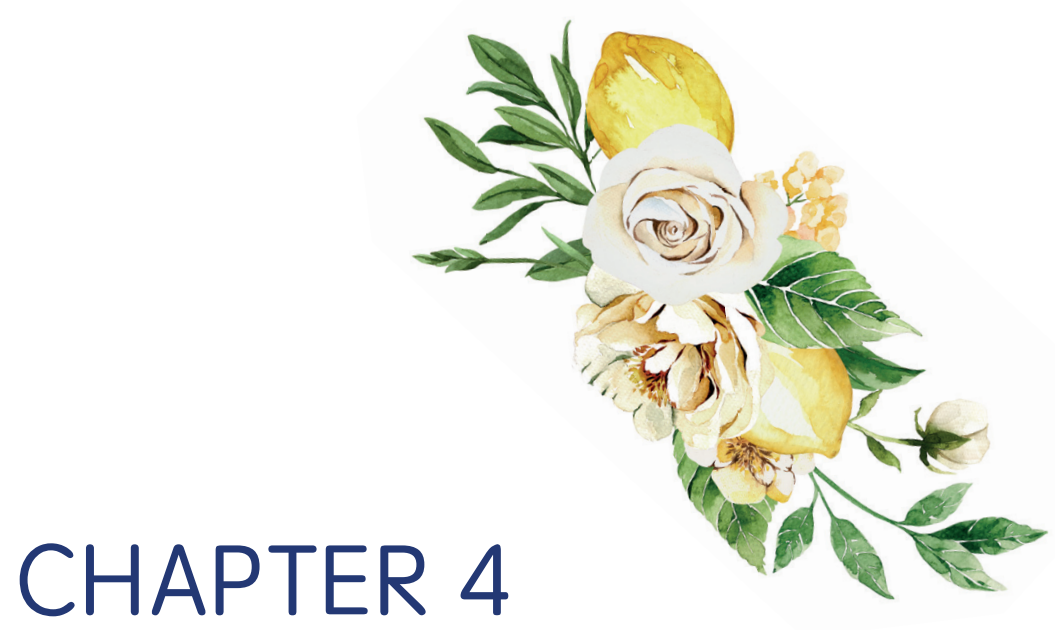

\section{Healthy aging and muscle function are positively associated with $\mathrm{NAD}^{+}$abundance in humans}

Lotte Grevendonk*, Georges E. Janssens*, Ruben Zapata-Perez, Bauke V. Schomakers, Johan de Vogel-van den Bosch, Jan M. W. Geurts, Michel van Weeghel, Patrick Schrauwen, Riekelt H. Houtkooper, Joris Hoeks.

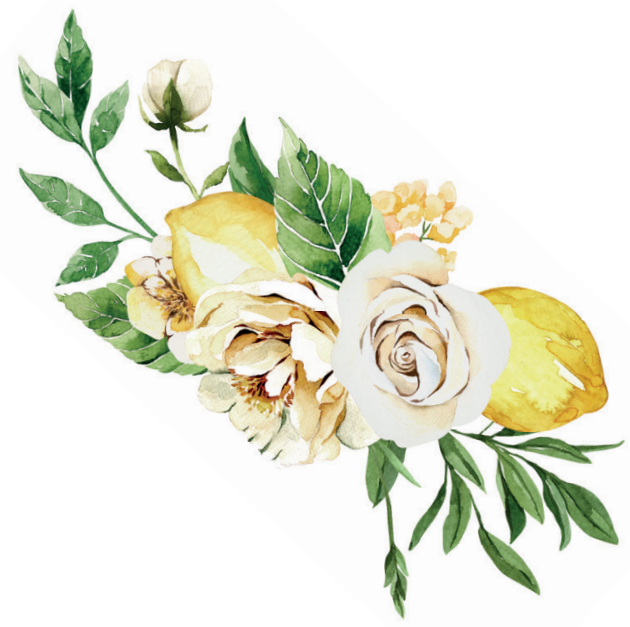

*Equal contribution 


\section{ABSTRACT}

Skeletal muscle is greatly affected by aging, resulting in a loss of metabolic and physical function. However, the underlying molecular processes and how (lack of) physical activity is involved in age-related metabolic decline in muscle function in humans is largely unknown. Here we investigated the muscle metabolome comparing young to older adults, whereby the older adults were either exercisetrained, had normal physical activity levels, or were physically impaired. Intriguingly, nicotinamide adenine dinucleotide $\left(\mathrm{NAD}^{+}\right)$was one of the most prominent metabolites to decline with age in human skeletal muscle, in line with preclinical models. This decline was exacerbated in impaired older individuals and was nearly fully prevented in the exercise-trained older individuals. $\mathrm{NAD}^{+}$was so intimately tied to muscle health levels that a correlation existed between muscle $\mathrm{NAD}^{+}$content, average steps-per-day, and mitochondrial and muscle functioning. Our work demonstrates the importance of exercise to promote healthy aging of human muscle and suggests a crucial role of $\mathrm{NAD}^{+}$therein

\section{Keywords}

Healthy longevity, muscle aging, metabolomics, exercise, NAD+ 


\section{Abbreviations}

KYNA Kynurenic acid

KYNU Kynurenine

NaAD Nicotinic acid adenine dinucleotide

$\mathrm{NAD}^{+} \quad$ Nicotinamide adenine dinucleotide

NAM Nicotinamide

NaMN

Nicotinic acid mononucleotide

NAMPT

Nicotinamide phosphoribosyltransferase

NMN

Nicotinamide mononucleotide

NR

Nicotinamide riboside

$\mathrm{NRH}$

Dihydronicotinamide riboside

PARPs

Poly(ADP-ribose) polymerases

PBMC

Peripheral blood mononuclear cell

PPP

Pentose phosphate pathway

PRPP

Phosphoribosyl pyrophosphate

QA

Quinolinic acid

SPPB

Short physical performance battery

Trp

\section{L-Tryptophan}

UPLC-HRMS Ultra-high performance liquid chromatography high-resolution mass spectrometry 


\section{INTRODUCTION}

Over the past few decades, remarkable achievements in healthcare have led to a dramatic increase in the average life expectancy in most economically developed countries $^{1,2}$. However, this increased lifespan is not paralleled by an increase in health span, as aging is accompanied by the development of various age-related pathologies including metabolic disorders ${ }^{3}$. Understanding and targeting the aging process itself, rather than treating the symptoms of these age-related pathologies, may be the most effective strategy to prevent or counteract aging-related health decline ${ }^{4}$.

The development of many age-related diseases has been associated with disturbances in mitochondrial metabolism, a hallmark of the aging process ${ }^{5}$. In that context, we and others have previously shown in both muscle and liver that pathways involved in oxidative phosphorylation (OXPHOS), fatty acid oxidation, and mitochondrial biogenesis are impaired in aged mice ${ }^{6,7}$. In addition, we also reported that aged $C$. elegans are characterized by a prominent accumulation of most fatty acid species, which appeared to be controlled, at least in part, by the mitochondrial metabolic regulator $\mathrm{AMPK}^{8}$. Together, these preclinical studies highlight that alterations in various metabolic pathways, particularly within mitochondrial metabolism, are linked to the aging process and could therefore explain age-related metabolic complications.

Skeletal muscle is highly affected by the aging process $^{9}$ and age-induced loss of skeletal muscle mass and function have been related to mobility impairments ${ }^{10,11}$, an increased risk of falls ${ }^{12,13}$ and physical frailty ${ }^{14}$. Aging is also associated with a decline in muscle metabolism, as exemplified by a decrease in insulin sensitivity, metabolic inflexibility, increased oxidative damage, and the occurrence of skeletal muscle mitochondrial dysfunction ${ }^{15-17}$. On the other hand, regular exercise positively impacts muscle metabolism and function, and regular exercise training has been shown to protect against aging-induced deterioration of muscle health ${ }^{18,19}$.

Based on these considerations, the aims of the present study were to investigate the muscle metabolome comparing young and aged individuals, and to identify how 
differences in the aged individuals may be associated to muscle and mitochondrial function. This comparison was expanded upon by evaluating changes in three groups of older individuals recruited according to their 'muscle health' states; aged individuals who 1) were exercise-trained ('trained' older adults), 2) who possessed normal physical activity abilities ('normal' older adults), or 3) who displayed an impaired physical function ('impaired' older adults). Our results show that nicotinamide adenine dinucleotide $\left(\mathrm{NAD}^{+}\right)$was one of the most depleted metabolites with age and that age-related $\mathrm{NAD}^{+}$depletion followed a 'healthy aging' trend, whereby physically impaired older individuals were marked by significantly lower $\mathrm{NAD}^{+}$levels and exercise-trained older individuals could maintain $\mathrm{NAD}^{+}$abundance to levels nearly as high as in the young. Furthermore, we found that in the older adults $\mathrm{NAD}^{+}$muscle abundance positively correlated with muscle and mitochondrial health parameters. Taken together, these results provide a first-time account that $\mathrm{NAD}^{+}$declines in aging human muscle and that $\mathrm{NAD}^{+}$abundance is directly associated to the healthy aging state of the individual. 


\section{RESULTS}

\section{The metabolome of human muscle aging}

In order to better understand which metabolites were associated to aging and to establish a 'healthy aging' muscle signature, we turned to an extensively characterized cohort of young and aged individuals, which we previously established (clinicaltrials.gov identifier NCT03666013). This mixed-gender cohort consisted of individuals that were either young $(n=12)$ or belonged to one of three aged groups; trained older adults $(n=17)$, older adults with normal physical activity levels $(n=17)$, or physically impaired older adults $(n=6)$. Participants were assigned to different study groups based on age, their levels of self-reported physical exercise training, and their levels of physical function. Participants were considered normally physically active if they completed no more than one structured exercise session per week. Older participants were considered exercise-trained if they engaged in at least three structured exercise sessions of at least one hour each per week for an uninterrupted period of at least one year prior to inclusion. Older participants were classified as physically impaired in case of a Short Physical Performance Battery (SPPB) score of $\leq 9$ (Table S1). Upon inclusion, further details on habitual physical activity levels were obtained using accelerometry. Over the course of five days, young individuals took an average of $\sim 10 \mathrm{~K}$ steps per day and spent $2.5 \%$ of their active time in highintensity activities (Figure 1A). The normal older adults group possessed similar ( $10 \mathrm{~K}$ steps/day and $2.2 \%$ active time in high-intensity activities), ensuring that changes observed between these groups were age-related rather than fitnessrelated. Meanwhile, trained older adults were more active ( $13 \mathrm{~K}$ steps/day on average) with more time spent in high-intensity activities (5.2\%), whereas the physically impaired older adults displayed a lower average daily step count ( 6K) and a lower amount of their active time spent in high-intensity activities (1.0\%) (Figure 1A).

Using the muscle biopsies collected from each participant in these groups, we performed ultra-high performance liquid chromatography coupled to high-resolution mass spectrometry (UPLC-HRMS)-based metabolomics (Table S2). The results were used to establish a baseline understanding of the differences occurring 
between young and older adults with normal and equivalent levels of physical activity, taking into account all 137 metabolites we were able to annotate. Comparing the muscle metabolomes of these two groups using a principal component analysis, we found a general separation between the groups to exist within the first two principal components, suggesting aging to impart large changes in the muscle metabolome (Figure S1A).

To better understand what metabolites were contributing to the differences occurring with aging, we compared the accumulated and depleted metabolites (Figure 1B, Figure S1B). We found a clear signature of changes to occur with normal aging (Figure 1B). Intriguingly, when considering the top five metabolites either accumulating or depleting in older vs. young adults with normal and equal physical activity, we found older adults to possess higher levels of ophthalmic acid, an oxidative stress marker 20,21 , and dihydroxyacetone-phosphate and 3methoxytyramine, two metabolite families negatively associated to mitochondrial respiration ${ }^{22,23}$. Moreover, we found a significant age-related decline of $\mathrm{NAD}^{+}$to occur with aging. Together these changes suggest an age-related change in oxidative metabolism in skeletal muscle (Figure 1C). 
A

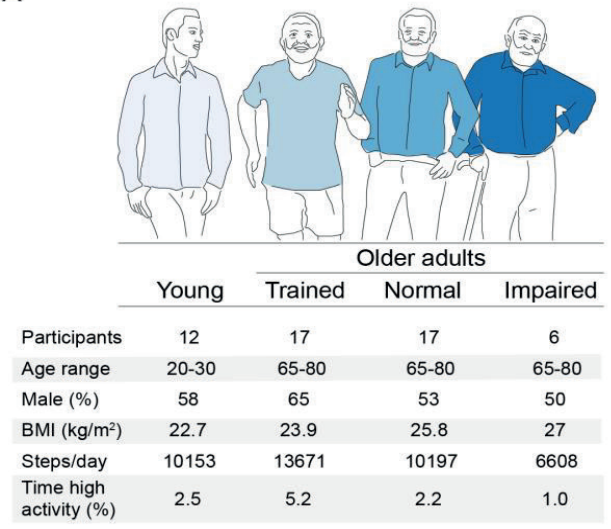

B

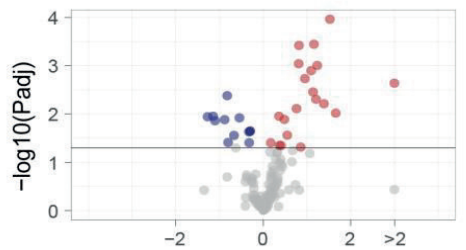

C

Normal older adult vs young (log2 fold change)

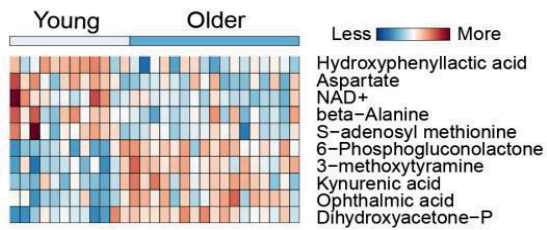

Figure 1: The metabolome of human muscle aging

(A) Study participants consisted of either young or older adults, whereby older adults were segmented into three categories: either 'exercise-trained,' 'normally physically active,' or 'physically impaired' with respect to their muscle health (Table S1). Group averages are presented for BMI, steps-per-day, and high-intensity physical active time, (B) Volcano plot of fold change (x-axis, log2 scale) versus $p$-value ( $y$-axis, -log10 scale) for older adults compared to young individuals with equal physical activity levels ('young' vs 'normal older adults')., illustrating significantly depleted (blue) or accumulated (red) metabolites with age. The horizontal line indicates significance $(p<0.05)$. Significance was determined using an empirical Bayes moderated t-test. (C) Top 5 accumulating and depleted metabolites in normal older adults compared to young, depicted as a heatmap with higher (red) or lower (blue) relative abundances of metabolites.

\section{$\mathrm{NAD}^{+}$abundance is directly related to healthy aging muscle}

Having established the metabolic changes occurring with aging in muscle, which pointed towards alterations in oxidative metabolism, we next asked which of these changes were not only linked to aging but were also related to muscle health levels. To do so, we ranked metabolites based on the degree to which their abundance level followed a health trend with the four groups, from young adults to trained older adults, to normally active older adults, to physically impaired older adults. This approach was designed to highlight metabolites that may decrease with normal aging, with a decline that can be either (partially) prevented by regular exercise training or exacerbated by belonging to a physically impaired aging group. Plotting 
these correlations against the normal aging changes served to highlight the agerelated changes that are important also on a health and exercise related axis. Remarkably, we found that changes in normal older adults and changes that follow a 'health trend' are highly correlated, implying that most metabolic changes that occur with age in the muscle can be reversed with regular exercise training (Figure 2A).

In line with this observation, we found that $\mathrm{NAD}^{+}$was not only one of the most depleted molecules in older adults when compared to young individuals, (Figure 1C) but also displayed the strongest association to healthy aging (Figure 2A). Indeed, age-related $\mathrm{NAD}^{+}$(Figure 1B) was exacerbated in the impaired older adults (Figure $2 \mathrm{~B}$, Figure $\mathrm{S} 2 \mathrm{~A}$ ). Furthermore, according to the Venn diagram analysis, a significant age-related decline in $\mathrm{NAD}^{+}$content was only found in common between the normal aging and the impaired aging groups (Figure S2B). Meanwhile, possessing an athletic lifestyle resulted in only a negligible age-related decline of $\mathrm{NAD}^{+}$in the trained older adults when compared to $\mathrm{NAD}^{+}$levels in the young (Figure 2B, Figure S2B).

Interestingly, we found ophthalmic acid, a marker of oxidative stress and one of the top-five accumulated metabolites (Figure 1C), to display the opposite trend as observed for $\mathrm{NAD}^{+}$, namely, an increase in older adults compared to young that became exacerbated in physically impaired older adults and was attenuated in trained older adults (Figure 2A, Figure 2C). In addition, we found that oxiglutathione, another marker of oxidative stress, followed the same trend as ophthalmic acid (Figure $2 \mathrm{~A}$ ), suggesting that $\mathrm{NAD}^{+}$depletion with aging may occur in parallel with increased ROS production. 
A

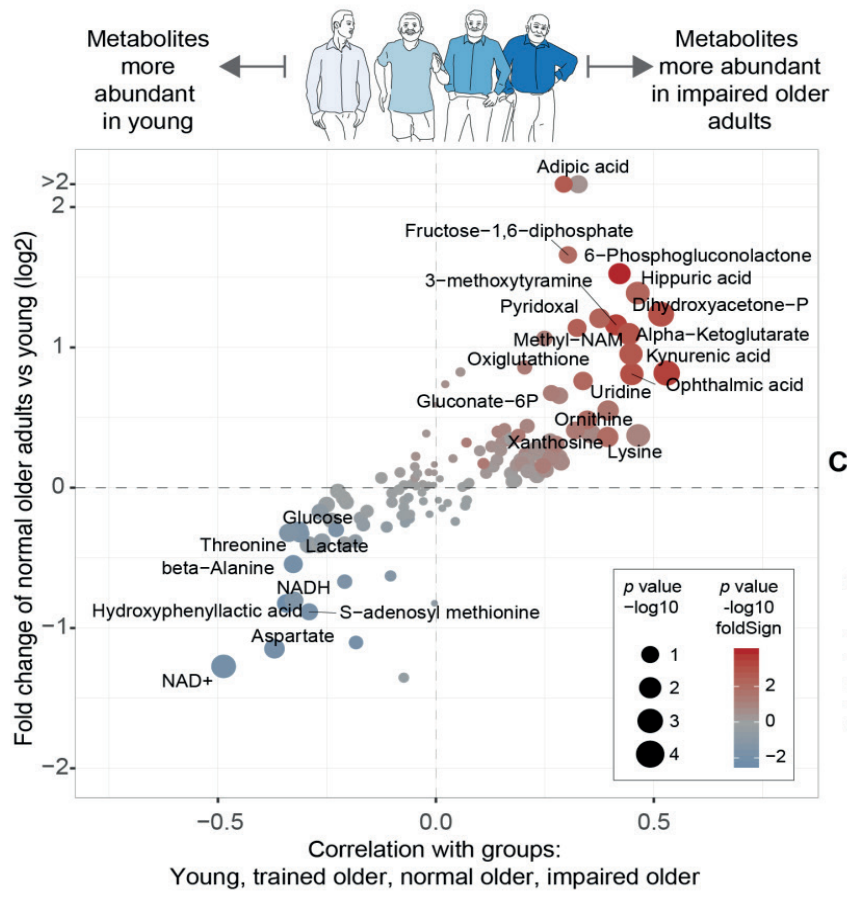

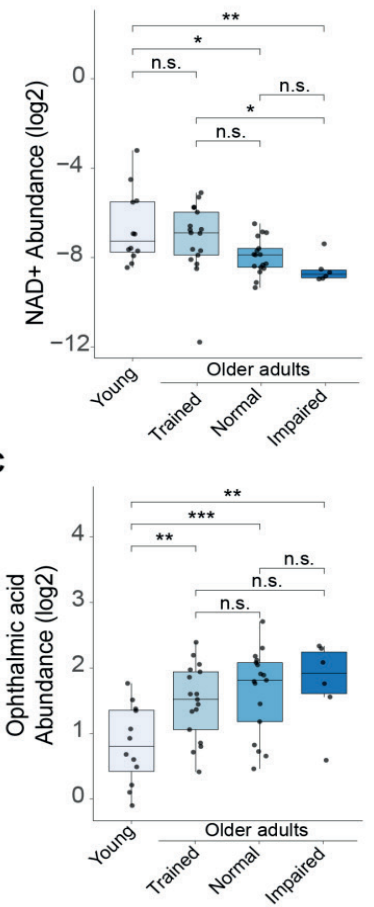

Figure 2: Muscle NAD+ levels relate to muscle health during aging

(A) Comparison of: (x-axis) the correlation a metabolite has to the four muscle health groups (young adults, trained older adults, older adults with normal physical activity levels, and physically impaired older adults), where the size of point on the graph indicates $p$-value of the correlation (- $\log 10$ scale), and correlation and significance were determined using Pearson's product-moment correlation coefficient, and (y-axis) the fold change the metabolite undergoes in older vs. young adults with normal physical activity, where the color of the point indicates the p-value of aging fold change (with directionality represented as either increasing, red, or decreasing, blue) and significance was determined using an empirical Bayes moderated t-test. The metabolite's name is depicted when the aging fold change had a significance of $p<0.05$. The graph reveals $N A D^{+}$to be both depleted in aging and to follow the strongest correlation to a healthy aging trend. (B) Abundance levels of $\mathrm{NAD}^{+}$in the four muscle health groups. (C) Abundance levels of ophthalmic acid in the four muscle health groups. Significance was determined using an empirical Bayes moderated ttest $\left({ }^{*} p<0.05,{ }^{* *} p<0.01,{ }^{* * *} p<0.001\right.$, n.s. $=$ not significant $)$.

\section{Modulation of the $\mathrm{NAD}^{+}$-pathway with aging}

Our untargeted metabolomics data pointed towards oxidative metabolism as a central player in aging muscle. Having observed that $\mathrm{NAD}^{+}$was both declined in 
normal aging and also possessed the strongest correlation to healthy aging, we next asked how the $\mathrm{NAD}^{+}$-pathway itself was modulated in our older adults relative to the young. To do so, we considered all $\mathrm{NAD}^{+}$-related metabolites (Figure S3A-L) and mapped out how they changed in each older adult group relative to young, onto the $\mathrm{NAD}^{+}$synthesis pathway (Figure 3 ). The depletion of $\mathrm{NAD}^{+}$, observed per aged group, was mirrored by a depletion of $\mathrm{NADH}$, although to a lesser extent. Furthermore, we observed a lack of modulation of $\mathrm{NADP}^{+}$and NADPH in the aged groups, which points towards an aging and exercise-dependent modulation of $\operatorname{NAD}(\mathrm{H})$, but not of their phosphorylated forms.

Having established $\mathrm{NAD}(\mathrm{H})$ 's associations to healthy aging, we next turned our attention to other metabolites involved in $\mathrm{NAD}^{+}$de novo synthesis and recycling. We found a strong correlation between kynurenic acid accumulation and the healthy aging trend (Figure 3, Figure S3B). Kynurenic acid is produced by kynurenine aminotransferase (KAT)-mediated irreversible transamination of kynurenine, a downstream metabolite of tryptophan in the $\mathrm{NAD}^{+}$de novo synthesis pathway, or through ROS-mediated oxidative degradation of kynurenine ${ }^{24}$. This latter explanation is in line with our previously noted increase in the oxidative damage markers ophthalmic acid and oxiglutathione ${ }^{20,21}$ in the older adults (Figure 2A, Figure 2C), supporting the hypothesis that kynurenic acid is produced via ROS-mediated degradation of kynurenine. 


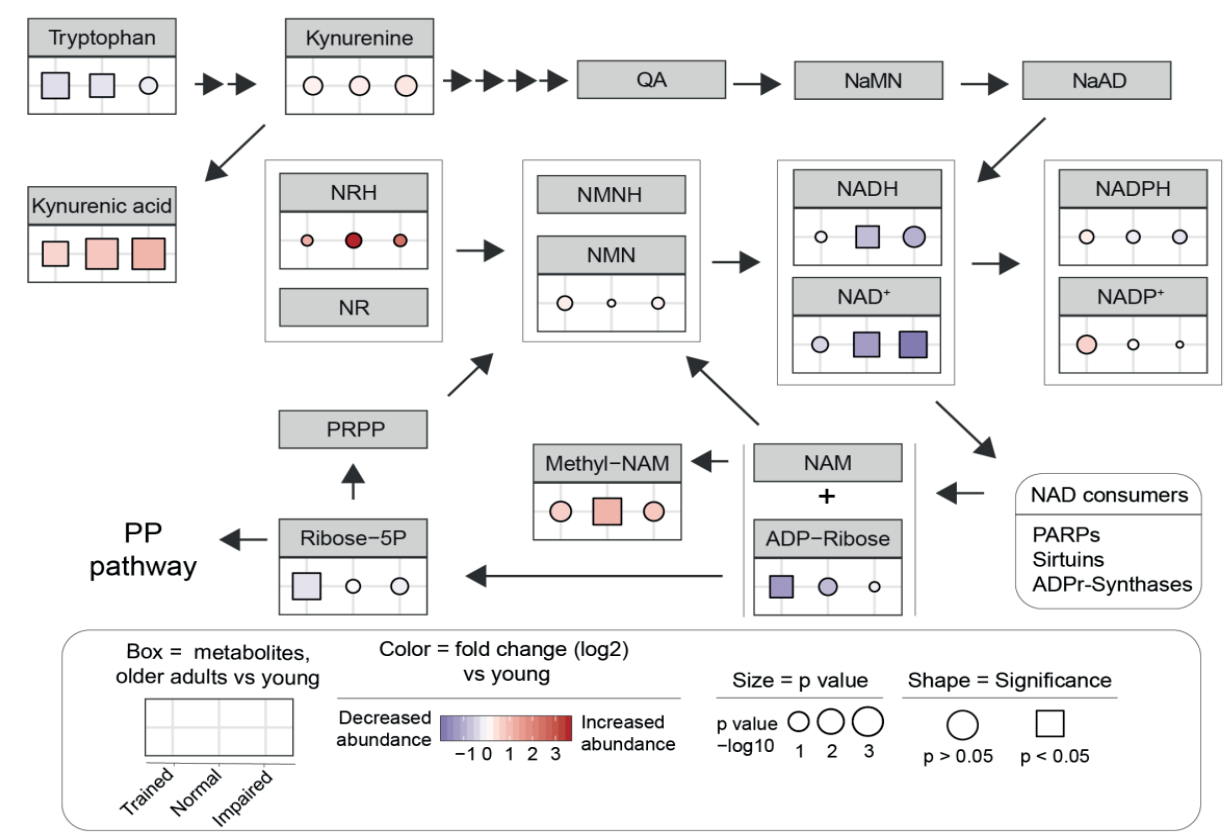

Figure 3: Major $\mathrm{NAD}^{+}$metabolites and changes in healthy muscle aging

Changes in the three older adult groups (trained, normally active, and physically impaired) relative to young are depicted for each $\mathrm{NAD}^{+}$-related metabolite in the map. The color indicates the fold change (blue=decreased, red=increased), the size indicates the $p$-value of the significance (-log10 scale), and the shape denotes a significance threshold (square, $p<0.05)$. Significance was determined using an empirical Bayes moderated t-test.

\section{$\mathrm{NAD}^{+}$metabolism is related to mitochondrial function and muscle health}

Our cohort possessed extensive phenotyping of muscle health parameters, which offered an unprecedented opportunity to better understand how the age-related decline of $\mathrm{NAD}^{+}$related to various outcomes of muscle health, an unexplored domain in human physiology. To address this, we performed a cross correlation of the abundances of $\mathrm{NAD}^{+}$-related metabolites to muscle-related parameters previously measured (clinicaltrials.gov identifier NCT03666013) in three older adult groups (Figure S4A). Phenotypical parameters consisted of molecular measurements of mitochondrial abundance (OXPHOS subunit abundances), mitochondrial respiration (oxygen consumption measurements in permeabilized muscle fibers), and in vivo mitochondrial function ( $\mathrm{PCr}$ recovery), in addition to physiological parameters, including muscle energetics (net exercise efficiency \%), 
muscle volume (MRI), muscle strength (isokinetic function), and habitual physical activity (accelerometry) (Figure S4B). By comparing and focusing our analyses on the metabolite abundances that correlate or anti-correlate with these molecular and physiological parameters, we reasoned that a correlation network could map the landscape of $\mathrm{NAD}^{+}$-related interactions associated with healthy muscle aging.

This approach revealed that a network existed that strongly linked $\mathrm{NAD}^{+}$metabolites to aging muscle function. Intriguingly, within this network kynurenic acid acted as a hub of negative associations, while $\mathrm{NAD}^{+}$was positively associated with many functional parameters of the muscle (Figure $4 \mathrm{~A}$ ). For example, we found that $\mathrm{NAD}^{+}$ abundance had a strong positive association to maximum ADP-stimulated (state 3 ) mitochondrial respiration (Figure 4B). Likewise, and quite remarkably, $\mathrm{NAD}^{+}$ abundance in muscle was also correlated to the average daily step count (Figure 4C). Meanwhile, when considering negative correlations, we observed that kynurenic acid was inversely associated with isokinetic muscle strength (Figure 4D) and kynurenine was inversely associated with net exercise efficiency (Figure 4E). Taken together, this approach revealed a first-time view of the significant relationship the $\mathrm{NAD}^{+}$pathway has to the healthy aging muscle functioning of older individuals.

Numerous preclinical studies have indicated $\mathrm{NAD}^{+}$depletion as the primary cause of disease and disability during aging ${ }^{25}$. While we have seen that boosting $N^{\prime} D^{+}$ metabolism in humans only induced very minor physiological changes ${ }^{26-28}$, increasing $\mathrm{NAD}^{+}$in animal models improved, amongst others, muscle recovery, endurance capacity, mitochondrial function, oxidative metabolism, insulin sensitivity, and lipid profiles, and extended lifespan ${ }^{17,29-35}$. In line with these preclinical studies, our $\mathrm{NAD}^{+}$-related metabolite and muscle physiology network supports that $\mathrm{NAD}^{+}$ abundance is indeed an indicator of muscle health status in aging individuals. 
A
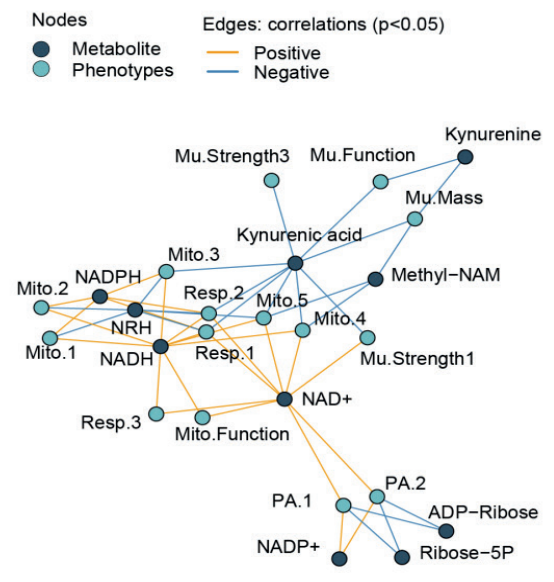

B
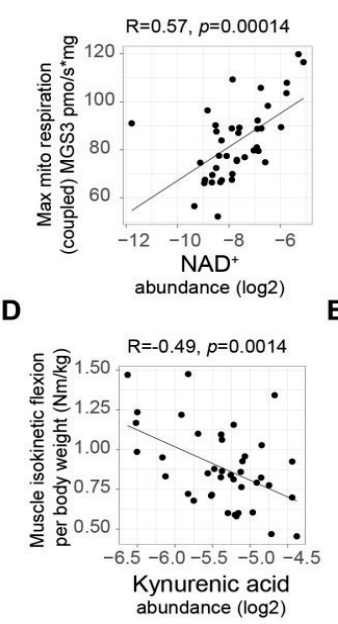

C
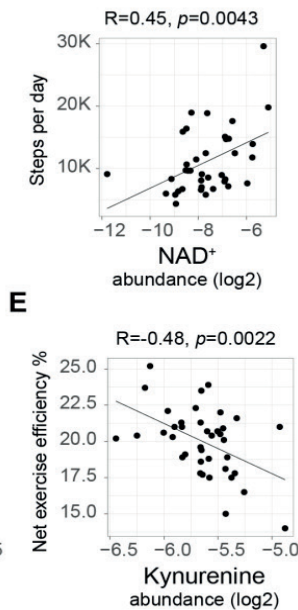

Figure 4: Molecular-physiological and $\mathrm{NAD}^{+}$-related healthy aging network

(A) Network of significant associations between molecular-physiological parameters (light blue nodes) and $\mathrm{NAD}^{+}$-related metabolites (dark blue nodes). Positive associations are in orange and negative associations in blue, where correlation and significance was determined in the older adults using Pearson's product-moment correlation coefficient $(p<0.05)$. (B-E) Examples of correlations within the network, highlighting the positive associations between (B) NAD ${ }^{+}$and mitochondrial respiration, and (C) $\mathrm{NAD}^{+}$and average daily steps, and highlighting the negative associations between (D) Kynurenic acid and muscle strength and (E) Kynurenine and exercise efficiency.

\section{CONCLUSIONS AND FUTURE PERSPECTIVES}

In the current study we investigated age-related muscle metabolomic changes comparing young and aged individuals. We identified how metabolomic differences in aged individuals relate to muscle and mitochondrial function by evaluating changes across a healthy (muscle) aging trend. Out of the 137 detected metabolites, $\mathrm{NAD}^{+}$was one of the most depleted metabolites upon aging, a finding which itself fills a void in the literature between preclinical animal research and clinical human research. Namely, to date, very few studies have established that $\mathrm{NAD}^{+}$, which declines in laboratory animals, also declines in human tissues ${ }^{36}$. Moreover, our study found that not only does $\mathrm{NAD}^{+}$decline with age, but also that its decline is associated with the health state of the aged individual. Specifically, it is more severely depleted in the impaired older adults, exercised-trained older adults nearly fully prevent this age-related loss, and $\mathrm{NAD}^{+}$abundance was directly positively 
associated with mitochondrial respiration and physiological muscle functioning when considering all individual older adults.

In this study, the young and normal aging groups were not engaged in structured exercise activities, and both recorded approximately 10,000 steps daily. Although this is well above the general recommendations ${ }^{37}$, our results suggest that maintaining a healthy physical activity level in older people comparable to the young is not sufficient to prevent an age-related decline in NAD+. However, endurancetrained older participants who had 12,500 steps/day and performed at least three exercise training sessions per week, did not show the decline in NAD ${ }^{+}$levels that occurred during normal aging. In contrast, physically impaired older adults in our study averaged less than 7,500 steps/day, reflecting a more sedentary lifestyle relative to the other participants ${ }^{38}$. The differences in habitual physical activity levels between the aging groups were directly reflected in $\mathrm{NAD}^{+}$metabolism, whereby quite strikingly, daily step counts correlated strongly with muscle NAD+ levels. Taken together, our results demonstrate that the best therapy to maintain youthful and physiologically important levels of $\mathrm{NAD}^{+}$during aging may simply involve highly structured exercise regimes, though this may not be realistic for all aging individuals.

While an age-related decline of $\mathrm{NAD}^{+}$and the benefits of boosting $\mathrm{NAD}^{+}$levels have been extensively demonstrated in model organisms ${ }^{17,29-35}$, relatively little evidence exists relating $\mathrm{NAD}^{+}$to aging and health in humans ${ }^{36}$. To our knowledge, to date, an age related decline in $\mathrm{NAD}^{+}$levels in humans has only been demonstrated to occur in the brain (using magnetic resonance spectroscopy) (39,40 $^{3}$ and pelvic skin samples (measured spectrophotometrically) ${ }^{41}$. Here we further demonstrate that $\mathrm{NAD}^{+}$ declines with human aging in skeletal muscle. Interestingly, it has previously been shown in human skeletal muscle that levels of nicotinamide phosphoribosyltransferase (NAMPT), the rate-limiting enzyme in the $\mathrm{NAD}^{+}$salvage pathway, decreases with aging and that both aerobic and resistance exercise training can increase NAMPT levels ${ }^{42}$. While the study was not designed to directly measure $\mathrm{NAD}^{+}$, the findings are in line with ours, suggesting that an aging lifestyle filled with exercise is efficient in influencing NAD ${ }^{+}$metabolic pathways. 
Transcriptomic and metabolomic studies in human skeletal muscle have extensively been studied in the context of frailty and sarcopenia, with key findings demonstrating changes in mitochondrial function, muscle growth, and cell turnover ${ }^{43-45}$. One study has demonstrated that $\mathrm{NAD}^{+}$(measured enzymatically) and mitochondrial oxidative capacity is decreased in sarcopenic older men relative to age-matched controls ${ }^{46}$. While the study was not designed to investigate whether older adults had lower levels of $\mathrm{NAD}^{+}$than young individuals in the first place, their findings are in line with our findings that impaired older adults have lower $\mathrm{NAD}^{+}$levels compared to normal older adults. Taken together, our work complements these recent findings and incorporates both young and old individuals, possessing both athletic and impaired aging states, to solidify our understanding of $\mathrm{NAD}^{+}$in skeletal muscle healthy aging.

In summary, our study advances our understanding of the relevance of $\mathrm{NAD}^{+}$ metabolism to human muscle aging, characterizing $\mathrm{NAD}^{+}$metabolism in extensively phenotyped young and older adults. Specifically, novel findings of our study include that (i) aging is associated with a depletion in skeletal muscle's NAD ${ }^{+}$abundance, occurring despite older adults maintaining adequate physical activity, (ii) lower NAD ${ }^{+}$ levels relate to impaired aging and a high intensity athletic aging lifestyle results in muscle $\mathrm{NAD}^{+}$levels similar to those seen in the young, and (iii) NAD+ metabolism strongly correlates with human skeletal muscle health during aging as assessed through mitochondrial and physical functioning, whereby the average daily step count an older adult takes directly associates with their muscle $\mathrm{NAD}^{+}$levels. Together this work affirms the relation of $\mathrm{NAD}^{+}$with overall health status in aging individuals and highlights the NAD-pathway as a promising target to promote healthy aging in humans.

\section{Acknowledgements}

L.G., J.H. and P.S. are financially supported by the TIFN research program Mitochondrial Health (ALWTF.2015.5) and the Netherlands Organization for Scientific Research (NWO). Work in the Houtkooper group is financially supported by an ERC Starting grant (no. 638290), a VIDI grant from ZonMw (no. 91715305), and a grant from the Velux Stiftung (no. 1063). G.E.J. is supported by a VENI grant from ZonMw. R.Z-P is supported by a post-doctoral grant from the European Union's 
Horizon 2020 research and innovation program under the Marie Skłodowska-Curie grant agreement number 840110 .

\section{Conflict of interest}

FrieslandCampina and Danone Nutricia Research are sponsors of the TIFN program and partly financed the project that led to human sample collection. They had no role in data collection, analysis, and decision to publish.

\section{Author contributions}

G.E.J., L.G., P.S., J.H., R.H.H. conceived the study. L.G. designed and performed the human clinical trial and experiments. G.E.J designed and performed the bioinformatics analyses. R.Z-P, B.V.S and M.v.W. performed the metabolomics analyses. J.M.W.G. and J.d.V.d.B reviewed the manuscript. G.E.J., L.G., R.Z-P, P.S. R.H.H and J.H. interpreted the results and wrote the manuscript with contributions from all other authors.

\section{METHODS}

Human subjects and procedures

Fifty-two participants, including 12 young ( 7 male and 5 female) and 40 older individuals (23 male and 17 female) were recruited in the community of Maastricht and its surroundings through advertisements at Maastricht University, in local newspapers, supermarkets, and at sports clubs. The study protocol was approved by the institutional Medical Ethical Committee and conducted in agreement with the declaration of Helsinki. All participants provided their written informed consent, and the study was registered at clinicaltrials.gov with identifier NCT03666013. Physiological data from this clinical trial has been reported in our previous study as part of a different analysis (manuscript accepted, Nature Communications 2021). Prior to inclusion, all subjects underwent a medical screening that included a physical examination by a physician and an assessment of physical function using the Short Physical Performance Battery (SPPB), comprised of a standing balance test, a 4-m walk test, and a chair-stand test. After the screening procedure, participants were assigned to the following study groups: Young individuals with normal physical activity (Y, 20 - 30 years), older adults with normal physical activity 
(NA, 65 - 80 years), athletic older adults (AA, $65-80$ years) and physically impaired older adults (IA, 65 - 80 years). Participants were considered normal, physically active (NA) if they completed no more than one structured exercise session per week. Participants were considered trained (AA) if they engaged in at least 3 structured exercise sessions of at least 1 hour each per week for an uninterrupted period of at least one year. Participants were classified as older adults with impaired physical function (IA) in case of an SPPB score of $\leq 9$. The SPPB score was calculated according to the cut-off points determined by ${ }^{47}$. Subject characteristics are summarized in Table S1.

\section{Muscle biopsy}

At 9 AM, after an overnight fast from 10 PM the preceding evening, a muscle biopsy was taken from the $m$. vastus lateralis under local anesthesia $(1.0 \%$ lidocaine without epinephrine) according to the Bergström method ${ }^{48}$. Part of the biopsy was immediately placed in an ice-cold preservation medium (BIOPS, OROBOROS Instruments, Innsbruck, Austria) and used for the preparation of permeabilized muscle fibers and measurement of ex vivo mitochondrial oxidative capacity, as described earlier 49,50 . The remaining part of the muscle biopsy was immediately frozen in melting isopentane and stored at $-80^{\circ} \mathrm{C}$ until further analysis.

\section{Mitochondrial respiration}

Permeabilized muscle fibers ( $2.5 \mathrm{mg}$ wet weight) were used for the assessment of mitochondrial capacity using a 2-chamber oxygraph (OROBOROS Instruments), according to ${ }^{51}$. To prevent oxygen limitation, the oxygraph chambers were hyperoxygenated up to $\sim 400 \mu \mathrm{mol} / \mathrm{L} \quad \mathrm{O}_{2}$. Subsequently, two different multisubstrate/inhibition protocols were used in which substrates and inhibitors were added consecutively at saturating concentrations. State 2 respiration was measured after the addition of malate $(4 \mathrm{mmol} / \mathrm{L})$ plus octanoyl-carnitine $(50 \mu \mathrm{mol} / \mathrm{L})$ or malate (4 mmol/L) plus glutamate $(10 \mathrm{mmol} / \mathrm{L})$. Subsequently, an excess of $2 \mathrm{mmol} / \mathrm{L}$ of ADP was added to determine coupled (state 3 ) respiration. Coupled respiration was then maximized with convergent electron input through Complex I and Complex II by adding succinate $(10 \mathrm{mmol} / \mathrm{L})$. Finally, the chemical uncoupler carbonylcyanide-4(trifluoromethoxy)-phenylhydrazone (FCCP) was titrated to assess the maximal capacity of the electron transport chain (state $3 u$ respiration). The integrity of the 
outer mitochondrial membrane was assessed by the addition of cytochrome C (10 $\mu \mathrm{mol} / \mathrm{L}$ ) upon maximal coupled respiration. If cytochrome $\mathrm{C}$ increased oxygen consumption $>15 \%$, the measurement was excluded to assure the viability and quality of the respiration measurement. All measurements were performed in quadruplicate.

\section{Mitochondrial content}

Mitochondrial content was assessed by mitochondrial OXPHOS protein expression using western blot analyses in Bioplex-lysates of human muscle tissue as previously described $^{52}$. Equal amounts of protein were loaded onto 4-12\% Bolt gradient gels (Novex, Thermo Fisher Scientific, Bleiswijk, The Netherlands). Proteins were transferred to a nitrocellulose membrane using the Trans-Blot Turbo transfer system (Bio-Rad Laboratories, Hercules, CA, USA). Primary antibodies contained a cocktail of mouse monoclonal antibodies directed against structural subunits of human OXPHOS (dilution 1:10,000; ab110411, Abcam, Cambridge, UK). The hOxPHOS proteins were detected using secondary antibodies conjugated with IRDye680 or IRDye800 and were quantified with a CLx Odyssey Near-Infrared Imager (Li-COR, Westburg, Leusden, The Netherlands).

Magnetic resonance imaging and spectroscopy (Pcr recovery and muscle volume) All MR experiments were performed on a 3T whole-body MRI scanner (Achieva 3TX; Philips Healthcare, Best, The Netherlands). Participants were positioned supine in the MR scanner to determine muscle volume with a series of T1-weighted images of the upper leg (slice thickness $=10.0 \mathrm{~mm}$, no gap between slices, in - plane resolution $=0.78 \times 0.78 \mathrm{~mm}$ ). A custom-written MATLAB 2016a script (The Mathworks Inc. Natick, MA, USA) was used to segment adipose tissue and muscle and quantify muscle volume semi-automatically. The muscle segmentation was performed in consecutive slices between the lower end of the $m$. rectus femoris and the lower end of the $m$. gluteus maximus. Phosphorus magnetic resonance spectroscopy $\left({ }^{31} \mathrm{P}\right.$ MRS) was performed to measure in vivo mitochondrial function in $m$. vastus lateralis, as previously described ${ }^{53}$, using a $6 \mathrm{~cm}$ surface coil. A series of 150 unlocalized ${ }^{31} \mathrm{P}$ spectra was acquired using the following parameters: single acquisitions (NSA = 1); repetition time $(T R)=4000 \mathrm{~ms}$; spectral bandwidth $=3000 \mathrm{~Hz}$; number of points = 
1024. The 150 spectra were divided into 10 spectra at rest, 70 spectra during kneeextension exercise, and 70 spectra during recovery. Exercise within the scanner was performed to an auditory cue $(0.5 \mathrm{~Hz})$ in a custom-built knee-extension device with adjustable weight. The intensity was chosen to correspond to $50-60 \%$ of the predetermined maximum weight. Spectra were analyzed with a custom-made MATLAB 2016a script. PCr, ATP, and inorganic phosphate peaks were fitted, and $\mathrm{pH}$ was determined. The $\mathrm{PCr}$ recovery was fitted with a mono-exponential function and the rate constant $\left(\mathrm{k}_{\left.\mathrm{in} \mathrm{s}^{-1}\right)}\right.$ was determined as previously described ${ }^{53}$. The rate constant $\mathrm{k}$ of $\mathrm{PCr}$ resynthesis is almost entirely dependent on ATP produced by oxidative phosphorylation and can be used as a parameter of in vivo oxidative capacity $^{54}$.

\section{Muscle function (exercise efficiency)}

Exercise efficiency was measured during a 1-hour submaximal exercise bout in the fasted state on an electronically-braked cycle ergometer. The submaximal cycle test was performed at $50 \%$ of Wmax, as determined during a maximal aerobic cycling test. To calculate exercise energy expenditure (EEE), $\mathrm{O}_{2}$ consumption and $\mathrm{CO}_{2}$ production were recorded using indirect calorimetry for 15 minutes at two timepoints, $\mathrm{T}=15$ and $\mathrm{T}=45$ (Omnical, IDEE, Maastricht, The Netherlands). EEE was calculated as the average of $T=15$ and $T=45$. Before the submaximal exercise test, resting energy expenditure (REE) was measured using indirect calorimetry. The Weir equation ${ }^{55}$ was used to calculate whole-body energy expenditure (EE). Net energy efficiency was computed as power output (watts converted to $\mathrm{kJ} / \mathrm{min}$ ) over EE during exercise (EEE) minus resting EE (REE) (Eq. 1) as described by ${ }^{56}$.

\section{Eq 1: NEE $(\%)=\left(\right.$ Work $(\mathrm{kJ} / \mathrm{min}) /(\mathrm{EEE}(\mathrm{kJ} / \mathrm{min})-\operatorname{REE}(\mathrm{kJ} / \mathrm{min})){ }^{*} 100$}

\section{Muscle strength}

Muscle contractile performance was measured using the Biodex System 3 Pro dynamometer (Biodex® Medical Systems, Inc., Shirley, NY, USA). The participants were stabilized in the device with shoulder, leg, and abdominal straps to prevent compensatory movement for the measurements. The test was performed with the left leg in all participants. To measure maximal muscle strength, each participant performed 30 consecutive knee extension and flexion movements (range of motion 
120 degrees/s). The peak torque of each extension and flexion was recorded. The maximal isokinetic knee-extensor and knee-flexor torque was defined as the highest peak torque and corrected for body weight $(\mathrm{Nm} / \mathrm{kg})$ and muscle volume $\left(\mathrm{Nm} / \mathrm{m}^{3}\right)$.

\section{Habitual physical activity}

Habitual physical activity was determined in all participants using an ActivPAL monitor (PAL Technologies, Glasgow, Scotland) for a consecutive period of 5 days, including two weekend days. Besides the total amount of steps per day, the total stepping time was calculated in proportion to waking time, determined according to ${ }^{57}$. Stepping time (i. e., physical activity) was then further classified into highintensity physical activity (HPA; minutes with a step frequency > 110 steps/min in proportion to waking time) and lower-intensity physical activity (LPA; minutes with a step frequency $\leq 110$ steps/min in proportion to waking time) $)^{37}$.

\section{Metabolomics}

Metabolomics was performed as previously described, with minor adjustments ${ }^{58}$. In a $2 \mathrm{~mL}$ tube, the following amounts of internal standard dissolved in water were added to each sample of approximately $5 \mathrm{mg}$ of freeze-dried muscle tissue: adenosine${ }^{15} \mathrm{~N}_{5}$-monophosphate $(5 \mathrm{nmol})$, adenosine- ${ }^{15} \mathrm{~N}_{5}$-triphosphate $(5 \mathrm{nmol}), \mathrm{D}_{4}$-alanine (0.5 nmol), D7-arginine (0.5 nmol), D3-aspartic acid $(0.5 \mathrm{nmol}), \mathrm{D}_{3}$-carnitine $(0.5$ $\mathrm{nmol}), \mathrm{D}_{4}$-citric acid $(0.5 \mathrm{nmol}),{ }^{13} \mathrm{C}_{1}$-citrulline $(0.5 \mathrm{nmol}),{ }^{13} \mathrm{C}_{6}$-fructose-1,6diphosphate (1 nmol), guanosine--15 $\mathrm{N}_{5}$-monophosphate $(5 \mathrm{nmol})$, guanosine- ${ }^{15} \mathrm{~N}_{5}$ triphosphate (5 nmol), ${ }^{13} \mathrm{C}_{6}$-glucose (10 nmol), ${ }^{13} \mathrm{C}_{6}$-glucose-6-phosphate (1 nmol), D3-glutamic acid $(0.5 \mathrm{nmol})$, D5-glutamine $(0.5 \mathrm{nmol})$, D5-glutathione $(1 \mathrm{nmol}),{ }^{13} \mathrm{C}_{6}$ isoleucine $(0.5 \mathrm{nmol}), \mathrm{D}_{3}$-lactic acid $(1 \mathrm{nmol})$, D3-leucine $(0.5 \mathrm{nmol})$, D4-lysine $(0.5$ $\mathrm{nmol})$, D3-methionine $(0.5 \mathrm{nmol})$, D6-ornithine $(0.5 \mathrm{nmol})$, D5-phenylalanine $(0.5$ $\mathrm{nmol}), \mathrm{D}_{7-p r o l i n e}(0.5 \mathrm{nmol}),{ }^{13} \mathrm{C}_{3}$-pyruvate $(0.5 \mathrm{nmol}), \mathrm{D}_{3}$-serine $(0.5 \mathrm{nmol}), \mathrm{D}_{6}-$ succinic acid $(0.5 \mathrm{nmol})$, D5-tryptophan $(0.5 \mathrm{nmol})$, D4-tyrosine $(0.5 \mathrm{nmol}), \mathrm{D}_{8}$-valine $(0.5 \mathrm{nmol})$. After adding the internal standard mix, a $5 \mathrm{~mm}$ stainless-steel bead and polar phase solvents (for a total of $500 \mu \mathrm{L}$ water and $500 \mu \mathrm{L} \mathrm{MeOH}$ ) were added and samples were homogenized using a TissueLyser II (Qiagen, Hilden, Germany) for $5 \mathrm{~min}$ at a frequency of 30 times/sec. Chloroform was added for a total of $1 \mathrm{~mL}$ to each sample before thorough mixing. Samples were then centrifuged for 10 minutes at $18.000 \mathrm{~g}$. The top layer, containing the polar phase, was transferred to a new 1.5 
$\mathrm{mL}$ tube and dried using a vacuum concentrator at $60^{\circ} \mathrm{C}$. Dried samples were reconstituted in $100 \mu \mathrm{L}$ 3:2 (v/v) methanol:water. Metabolites were analyzed using a Waters Acquity ultra-high performance liquid chromatography system coupled to a Bruker Impact II UM Ultra-High Resolution Qq-Time-Of-Flight mass spectrometer. Samples were kept at $12^{\circ} \mathrm{C}$ during analysis and $5 \mu \mathrm{L}$ of each sample was injected. Chromatographic separation was achieved using a Merck Millipore SeQuant ZICcHILIC column (PEEK $100 \times 2.1 \mathrm{~mm}, 3 \mu \mathrm{m}$ particle size). Column temperature was held at $30^{\circ} \mathrm{C}$. Mobile phase consisted of (A) 1:9 (v/v) acetonitrile:water and (B) 9:1 ( $\mathrm{v} / \mathrm{v})$ acetonitrile:water, both containing $5 \mathrm{mmol} / \mathrm{L}$ ammonium acetate. Using a flow rate of $0.25 \mathrm{~mL} / \mathrm{min}$, the LC gradient consisted of: $100 \% \mathrm{~B}$ for $0-2 \mathrm{~min}$, reach $0 \% \mathrm{~B}$ at $28 \mathrm{~min}, 0 \%$ B for $28-30 \mathrm{~min}$, reach $100 \%$ B at $31 \mathrm{~min}, 100 \%$ B for 31-32 min. Column re-equilibration is achieved at a flow rate of $0.4 \mathrm{~mL} / \mathrm{min}$ at $100 \% \mathrm{~B}$ for $32-35 \mathrm{~min}$. MS data were acquired using negative and positive ionization in full scan mode over the range of $\mathrm{m} / \mathrm{z}$ 50-1200. Data were analyzed using Bruker TASQ software version 2.1.22.3. All reported metabolite intensities were normalized to dry tissue weight, as well as to internal standards with comparable retention times and response in the MS. Metabolite identification has been based on a combination of accurate mass, (relative) retention times and fragmentation spectra, compared to the analysis of a library of standards (Sigma-Aldrich MSMLS).

\section{Statistical analyses and data visualization}

Data was processed and analyses were performed with $\mathrm{R}$ version $3.5 .1^{59}$ and Bioconductor version 3.760. Data was processed in part with the $\mathrm{R}$ package dplyr version 1.0.261. Principal Component Analysis (PCA) was performed using the $R$ package MixOmics version 6.6.262. Significance was assessed using an empirical Bayes moderated t-test on log2 transformed data within limma's linear model framework, taking participant groups into account ${ }^{63,64}$. Networks were constructed and visualized using igraph version 1.2.4.265. Unless implemented through an aforementioned $\mathrm{R}$ package or base $\mathrm{R}$ graphics, visualization of data was performed using ggplot2 version 3.2.166, ggpubr v 0.2.567, ggrepel version $0.8 .1^{68}$, with colors from RColorBrewer version 1.1-2 ${ }^{69}$. 


\section{Data availability}

Metabolomics data are available as supplementary materials accompanying this manuscript as both summary statistics and processed abundance values per individual. 


\section{SUPPLEMENTARY MATERIAL FOR CHAPTER 4}

\begin{tabular}{|c|c|c|c|}
\hline $\begin{array}{c}\text { Young adults, } \\
\text { normal physical } \\
\text { activity }\end{array}$ & $\begin{array}{l}\text { Trained older } \\
\text { adults }\end{array}$ & $\begin{array}{c}\text { Older adults, normal } \\
\text { physical activity }\end{array}$ & $\begin{array}{l}\text { Physically impaired } \\
\text { older adults }\end{array}$ \\
\hline 20-30 years old & \multicolumn{3}{|c|}{$65-80$ years old } \\
\hline $\begin{array}{c}\text { Normal physical } \\
\text { activity }\end{array}$ & $\begin{array}{l}\text { Highly physically } \\
\text { active }\end{array}$ & \multicolumn{2}{|c|}{ Normal physical activity } \\
\hline $\begin{array}{c}\text { Max } 1 \mathrm{~h} \text { exercise } \\
\text { per week }\end{array}$ & $\begin{array}{c}\geq 3 \text { times exercise } \\
\text { per week }\end{array}$ & \multicolumn{2}{|c|}{ Max $1 \mathrm{~h}$ exercise per week } \\
\hline \multicolumn{3}{|c|}{$\begin{array}{c}\text { Normal physical function } \\
\text { SPPB score }>9\end{array}$} & $\begin{array}{l}\text { Impaired physical } \\
\text { function } \\
\text { SPPB score } \leq 9\end{array}$ \\
\hline
\end{tabular}

Table S1: Inclusion criteria per study group. Participants were assigned to different study groups based on age, self-reported exercise levels, and physical function. Participants were considered normally physically active if they completed no more than one structured exercise session per week. Participants were considered exercise-trained if they engaged in at least three structured exercise sessions of at least 1 hour each per week for an uninterrupted period of at least one year prior to inclusion. Physical function was measured using the Short Physical Performance Battery, which includes a standing balance test, a 4-m walk test, and a chair-stand test. The SPPB score was calculated according to the cut-off points determined by (Guralnik et al. 1994). Participants were classified as physically impaired in case of an SPPB score of $\leq 9$. 
A

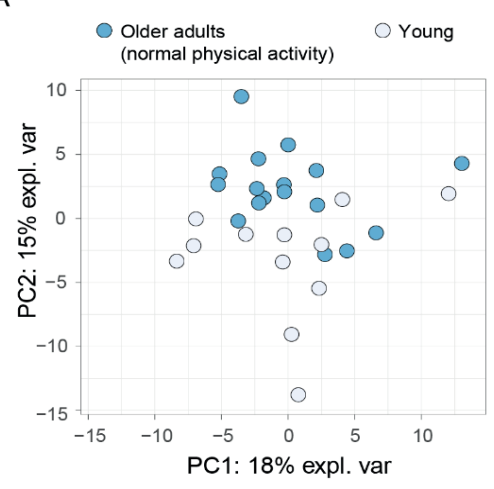

B

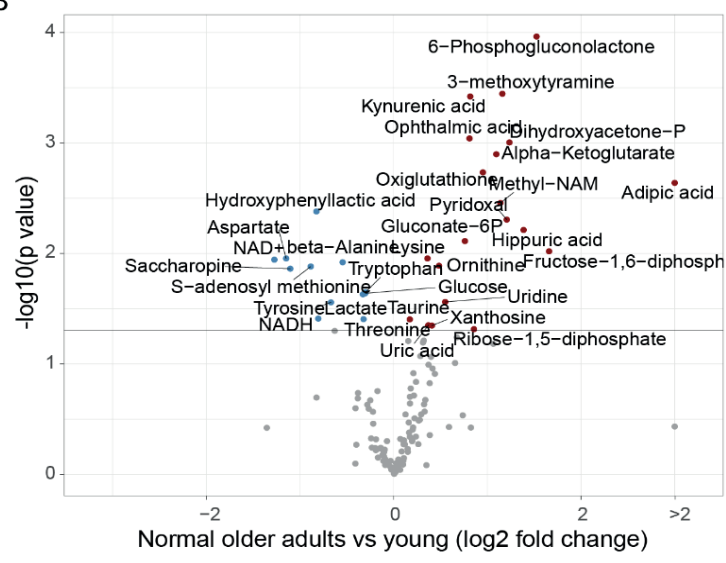

Figure S1: Global metabolomics changes of human muscle aging

(A) Principal Component Analysis (PCA) of metabolomes of young and older individuals possessing equal physical activity levels ('young' vs 'normal older adults'). (B) Volcano plot of fold change (x-axis, log2 scale) versus $p$-value ( $y$-axis, -log10 scale) for older adults compared to young individuals with equal physical activity levels, illustrating significantly depleted (blue) or accumulated (red) metabolites with age. The horizontal line indicates significance $(p<0.05)$. Significance was determined using an empirical Bayes moderated ttest. 
A

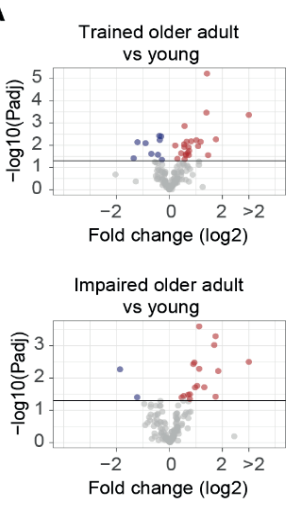

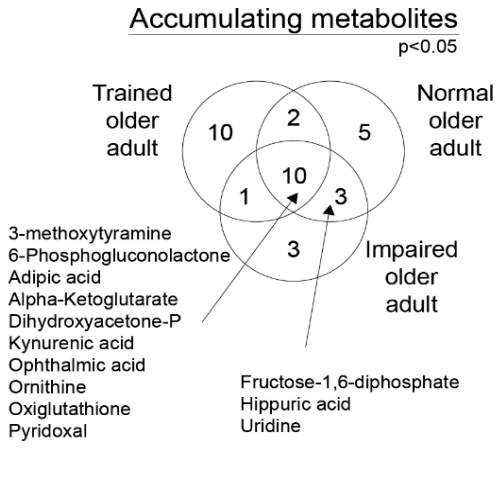

Depleted metabolites

$p<0.05$

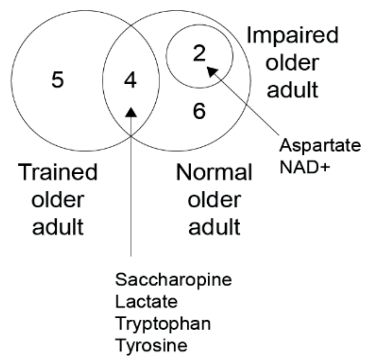

\section{Figure S2: Comparison of age-related changes in each aging group}

(A) Volcano plots of fold change (x-axis, log2 scale) versus $p$-value (y-axis, -log10 scale) for trained older adults (top), and physically impaired older adults (bottom) compared to young individuals, illustrating significantly depleted (blue) or accumulated (red) metabolites with age. Line indicates significance $(p<0.05)$. Significance was determined using an empirical Bayes moderated t-test. (B) Venn diagram of the overlap of significantly accumulating or depleting metabolites in each aged group (trained older adults, older adults with normal physical activity levels, physically impaired older adults) compared to young individuals ( $p<$ 0.05). 
A

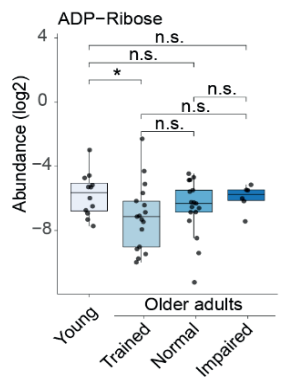

D

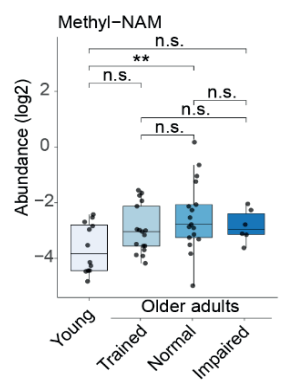

G

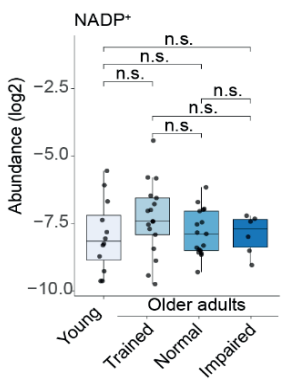

J

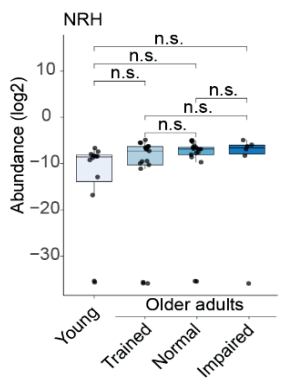

B

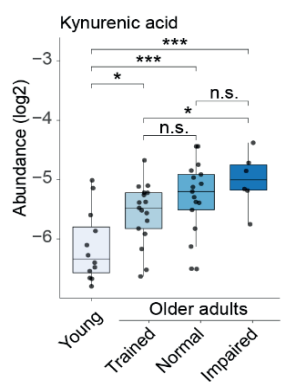

E

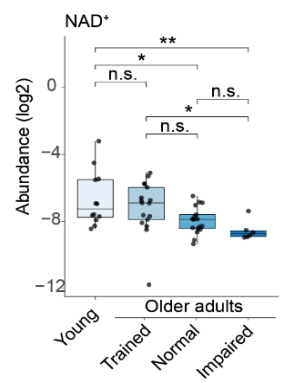

H

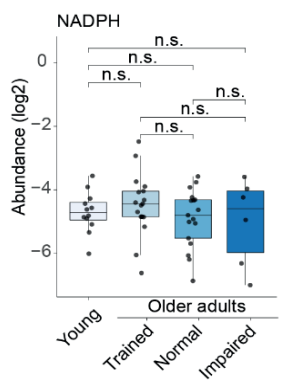

K

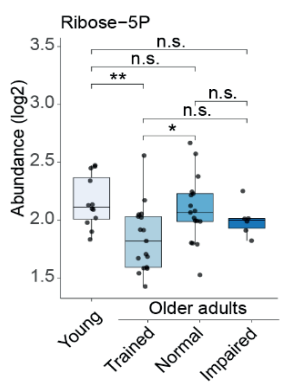

C

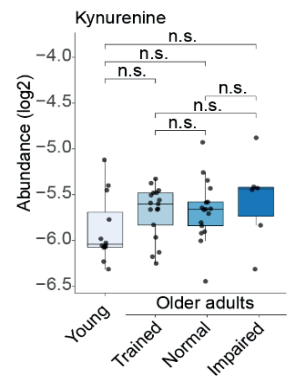

F

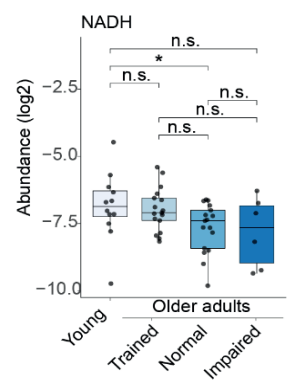

I

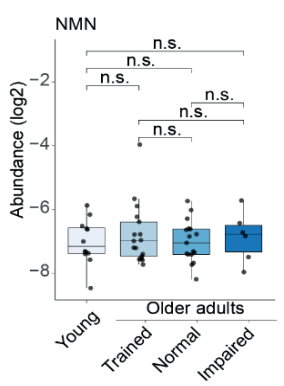

L

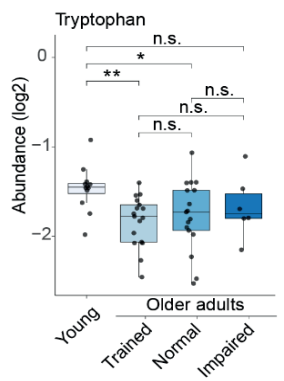

Figure S3: NAD+-related metabolites of healthy aging groups (A-L) Abundance levels of $\mathrm{NAD}^{+}$-related metabolites in the four muscle health groups, for (A) ADP-ribose, (B) Kynurenic acid, (C) Kynurenine, (D) Methyl-NAM, (E) NAD , (F) NADH, (G) NADP ${ }^{+}$, (H) $\mathrm{NADPH}$, (I) Nicotinamide mononucleotide (NMN), (J) Dihydronicotinamide riboside (NRH), (K) Ribose-5P, and (L) Tryptophan. Significance was determined using an empirical Bayes moderated t-test $\left({ }^{*} p<0.05,{ }^{* *} p<0.01,{ }^{* * *} p<0.001\right.$, n.s. $=$ not significant). 
A
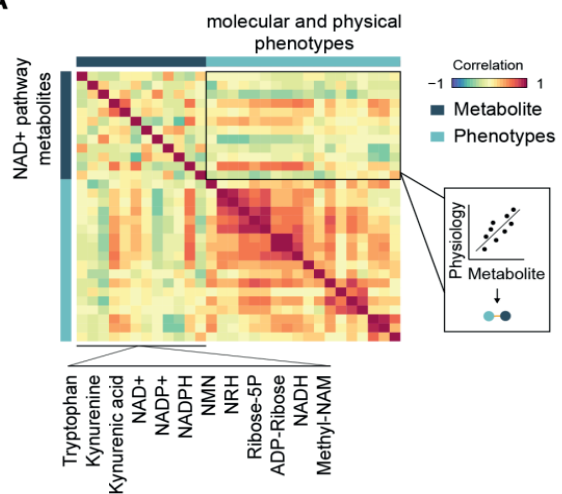

B

$\frac{\text { Node }}{\text { Mu.Function }} \frac{\text { Measurement }}{\text { Muscle function - Net exercise efficiency }(\%)}$

$\begin{array}{ll}\text { Mu.Function } & \text { Muscle function - Net exercise efficiency (\%) } \\ \text { Mito.1 } & \text { Mitochondria - OXPHOS compl V blot (A.U.) }\end{array}$

$\begin{array}{ll}\text { Mito.1 } & \text { Mitochondria - OXPHOS compl V blot (A.U.) } \\ \text { Mito.2 } & \text { Mitochondria - OXPHOS compl III blot (A.U.) }\end{array}$

Mito.3 Mitochondria - OXPHOS compl II blot (A.U.)

Mito.4 Mitochondria - OXPHOS compl IV blot (A.U.)

Mito.5 Mitochondria - OXPHOS compl I blot (A.U.)

Resp.1 Respiration - Coupled MOGS3 $\left(\mathrm{pmol} /\left(\mathrm{s}^{*} \mathrm{mg}\right)\right.$ )

Resp.2 Respiration - Uncoupled MOGS (pmol/( $\left.\mathbf{s}^{\star} \mathrm{mg}\right)$ )

Resp.3 Respiration - Coupled MGS3 (pmol/( $\left.\mathrm{s}^{*} \mathrm{mg}\right)$ )

Mito.Function Mitochondrial function - MRS $\mathrm{PCr}$ recovery rate

Mu.Mass Muscle mass - MRI Muscle volume $(\mathrm{cm} 3)$

Mu.Strength1 Muscle isokinetic extension / body weight $(\mathrm{Nm} / \mathrm{kg})$

Mu.Strength2 Muscle isokinetic extension / muscle volume $(\mathrm{Nm} / \mathrm{cm} 3)$

Mu.Strength3 Muscle isokinetic flexion / body weight $(\mathrm{Nm} / \mathrm{kg})$

Mu.Strength4 Muscle isokinetic flexion / muscle volume $(\mathrm{Nm} / \mathrm{cm} 3)$

PA.1 Physical activity - steps per day

PA 2 Physical activity- $\%$ time high physical activity

PA. 3

Physical activity- \% time low physical activity

Figure S4: Correlation of molecular-physiological phenotypes with metabolites involved in NAD+ synthesis

(A) Correlation matrix comparing the paired metabolome (dark blue) and muscle health parameters (light blue) in the older adults (Pearson's product-moment correlation coefficient). The scale ranges from blue (negative correlation), to yellow (no correlation), to red (positive correlation). Inset and cartoon: correlation between metabolites and muscle health parameters are used to reconstruct the network. (B) Abbreviations and measurements of the molecular-physiological phenotypes assessed in this analysis. Note: MOGS3, state 3 respiration upon malate + octanoyl-carnitine + glutamate + succinate, MGS3, state 3 respiration upon malate + glutamate + succinate 


\section{REFERENCES}

1. Salomon, J. A. et al. Healthy life expectancy for 187 countries, 1990-2010: A systematic analysis for the Global Burden Disease Study 2010. Lancet (2012). doi:10.1016/S0140-6736(12)61690-0

2. Ferrucci, L., Giallauria, F. \& Guralnik, J. M. Epidemiology of Aging. Radiologic Clinics of North America (2008). doi:10.1016/j.rcl.2008.07.005

3. Butler, R. N. et al. New model of health promotion and disease prevention for the $21 \mathrm{st}$ century. BMJ (2008). doi:10.1136/bmj.a399

4. Niccoli, T. \& Partridge, L. Ageing as a risk factor for disease. Current Biology 22, (2012).

5. López-Otín, C., Blasco, M. A., Partridge, L., Serrano, M. \& Kroemer, G. The hallmarks of aging. Cell 153, (2013).

6. Houtkooper, R. H. et al. The metabolic footprint of aging in mice. Sci. Rep. 1, 134 (2011).

7. Uchitomi, R. et al. Metabolomic Analysis of Skeletal Muscle in Aged Mice. Sci. Rep. (2019). doi:10.1038/s41598-019-46929-8

8. Gao, A. W. et al. A sensitive mass spectrometry platform identifies metabolic changes of life history traits in C. elegans. Sci. Rep. 7, (2017).

9. Roubenoff, R. Sarcopenia and its implications for the elderly. Eur. J. Clin. Nutr. (2000). doi:10.1038/sj.ejcn.1601024

10. Janssen, I., Heymsfield, S. B. \& Ross, R. Low relative skeletal muscle mass (sarcopenia) in older persons is associated with functional impairment and physical disability. J. Am. Geriatr. Soc. (2002). doi:10.1046/j.1532-5415.2002.50216.x

11. Distefano, G. et al. Physical activity unveils the relationship between mitochondrial energetics, muscle quality, and physical function in older adults. J. Cachexia. Sarcopenia Muscle (2018). doi:10.1002/jcsm.12272

12. Talbot, L. A., Musiol, R. J., Witham, E. K. \& Metter, E. J. Falls in young, middle-aged and older community dwelling adults: Perceived cause, environmental factors and injury. BMC Public Health (2005). doi:10.1186/1471-2458-5-86

13. Gadelha, A. B., Neri, S. G. R., Bottaro, M. \& Lima, R. M. The relationship between muscle quality and incidence of falls in older community-dwelling women: An 18month follow-up study. Exp. Gerontol. (2018). doi:10.1016/j.exger.2018.06.018

14. Fried, L. P. et al. Frailty in older adults: Evidence for a phenotype. Journals Gerontol. Ser. A Biol. Sci. Med. Sci. (2001). doi:10.1093/gerona/56.3.m146

15. Amati, F. et al. Physical inactivity and obesity underlie the insulin resistance of aging. Diabetes Care (2009). doi:10.2337/dc09-0267

16. Riera, C. E. \& Dillin, A. Tipping the metabolic scales towards increased longevity in mammals. Nat. Cell Biol. 17, 196-203 (2015).

17. Romani, M. et al. NAD+ boosting reduces age-associated amyloidosis and restores mitochondrial homeostasis in muscle. Cell Rep. 34, 108660 (2021).

18. Cartee, G. D., Hepple, R. T., Bamman, M. M. \& Zierath, J. R. Exercise Promotes Healthy Aging of Skeletal Muscle. Cell Metabolism 23, 1034-1047 (2016).

19. Greggio, C. et al. Enhanced Respiratory Chain Supercomplex Formation in Response to Exercise in Human Skeletal Muscle. Cell Metab. 25, 301-311 (2017).

20. Soga, T. et al. Differential metabolomics reveals ophthalmic acid as an oxidative stress biomarker indicating hepatic glutathione consumption. J. Biol. Chem. (2006). doi:10.1074/jbc.M601876200 
21. Dello, S. A. W. G. et al. Systematic review of ophthalmate as a novel biomarker of hepatic glutathione depletion. Clinical Nutrition (2013). doi:10.1016/j.clnu.2012.10.008

22. Smith, K. R., Hayat, F., Andrews, J. F., Migaud, M. E. \& Gassman, N. R. Dihydroxyacetone Exposure Alters $\mathrm{NAD}(\mathrm{P}) \mathrm{H}$ and Induces Mitochondrial Stress and Autophagy in HEK293T Cells. Chem. Res. Toxicol. (2019). doi:10.1021/acs.chemrestox.9b00230

23. Gluck, M. R. \& Zeevalk, G. D. Inhibition of brain mitochondrial respiration by dopamine and its metabolites: Implications for Parkinson's disease and catecholamine-associated diseases. J. Neurochem. (2004). doi:10.1111/j.14714159.2004.02747.x

24. Wirthgen, E., Hoeflich, A., Rebl, A. \& Günther, J. Kynurenic Acid: The Janus-faced role of an immunomodulatory tryptophan metabolite and its link to pathological conditions. Frontiers in Immunology (2018). doi:10.3389/fimmu.2017.01957

25. Rajman, L., Chwalek, K. \& Sinclair, D. A. Therapeutic Potential of NAD-Boosting Molecules: The In Vivo Evidence. Cell Metabolism (2018). doi:10.1016/j.cmet.2018.02.011

26. Remie, C. M. E. et al. Nicotinamide riboside supplementation alters body composition and skeletal muscle acetylcarnitine concentrations in healthy obese humans. Am. J. Clin. Nutr. (2020). doi:10.1093/ajcn/nqaa072

27. Pirinen, E. et al. Clinical and Translational Report Niacin Cures Systemic NAD + Deficiency and Improves Muscle Performance in Adult-Onset Mitochondrial Myopathy Niacin Cures Systemic NAD + Deficiency and Improves Muscle Performance in AdultOnset Mitochondrial Myopathy. Cell Metab. 31, (2020).

28. Elhassan, Y. S. et al. Nicotinamide Riboside Augments the Aged Human Skeletal Muscle NAD+ Metabolome and Induces Transcriptomic and Anti-inflammatory Signatures. Cell Rep. 28, 1717-1728.e6 (2019).

29. Cantó, C. et al. The NAD+ precursor nicotinamide riboside enhances oxidative metabolism and protects against high-fat diet-induced obesity. Cell Metab. (2012). doi:10.1016/j.cmet.2012.04.022

30. Sorrentino, V. et al. Enhancing mitochondrial proteostasis reduces amyloid- $\beta$ proteotoxicity. Nature 552, 187-193 (2017).

31. Zhang, $\mathrm{H}$. et al. NAD+ repletion improves mitochondrial and stem cell function and enhances life span in mice. Science (80-. ). (2016). doi:10.1126/science.aaf2693

32. Gomes, A. P. et al. Declining NAD+ induces a pseudohypoxic state disrupting nuclear-mitochondrial communication during aging. Cell (2013). doi:10.1016/j.cell.2013.11.037

33. Mills, K. F. et al. Long-Term Administration of Nicotinamide Mononucleotide Mitigates Age-Associated Physiological Decline in Mice. Cell Metab. (2016). doi:10.1016/j.cmet.2016.09.013

34. Yoshino, J., Mills, K. F., Yoon, M. J. \& Imai, S. I. Nicotinamide mononucleotide, a key $\mathrm{NAD}+$ intermediate, treats the pathophysiology of diet- and age-induced diabetes in mice. Cell Metab. (2011). doi:10.1016/j.cmet.2011.08.014

35. Mouchiroud, L. et al. The NAD+/sirtuin pathway modulates longevity through activation of mitochondrial UPR and FOXO signaling. Cell (2013). doi:10.1016/j.cell.2013.06.016

36. Connell, N. J., Houtkooper, R. H. \& Schrauwen, P. NAD + metabolism as a target for metabolic health: have we found the silver bullet? Diabetologia (2019). doi:10.1007/s00125-019-4831-3 
37. Tudor-Locke, C. et al. How many steps/day are enough? for adults. Int. J. Behav. Nutr. Phys. Act. (2011). doi:10.1186/1479-5868-8-79

38. Tudor-Locke, C., Hatano, Y., Pangrazi, R. P. \& Kang, M. Revisiting 'how many steps are enough?' Med. Sci. Sports Exerc. (2008). doi:10.1249/MSS.0b013e31817c7133

39. Zhu, X. H., Lu, M., Lee, B. Y., Ugurbil, K. \& Chen, W. In vivo NAD assay reveals the intracellular NAD contents and redox state in healthy human brain and their age dependences. Proc. Natl. Acad. Sci. U. S. A. (2015). doi:10.1073/pnas.1417921112

40. Cuenoud, B. et al. Brain NAD Is Associated With ATP Energy Production and Membrane Phospholipid Turnover in Humans. Front. Aging Neurosci. (2020). doi:10.3389/fnagi.2020.609517

41. Massudi, H. et al. Age-associated changes in oxidative stress and NAD+ metabolism in human tissue. PLoS One (2012). doi:10.1371/journal.pone.0042357

42. de Guia, R. M. et al. Aerobic and resistance exercise training reverses age-dependent decline in NAD+ salvage capacity in human skeletal muscle. Physiol. Rep. (2019). doi:10.14814/phy2.14139

43. Drummond, M. J. et al. Aging and microRNA expression in human skeletal muscle: A microarray and bioinformatics analysis. Physiol. Genomics (2011). doi:10.1152/physiolgenomics.00148.2010

44. Fazelzadeh, P. et al. The Muscle Metabolome Differs between Healthy and Frail Older Adults. J. Proteome Res. (2016). doi:10.1021/acs.jproteome.5b00840

45. Rivas, D. A. et al. Diminished skeletal muscle microRNA expression with aging is associated with attenuated muscle plasticity and inhibition of IGF-1 signaling. FASEB J. (2014). doi:10.1096/fj.14-254490

46. Migliavacca, E. et al. Mitochondrial oxidative capacity and NAD+ biosynthesis are reduced in human sarcopenia across ethnicities. Nat. Commun. (2019). doi:10.1038/s41467-019-13694-1

47. Guralnik, J. M. et al. A short physical performance battery assessing lower extremity function: Association with self-reported disability and prediction of mortality and nursing home admission. Journals Gerontol. (1994). doi:10.1093/geronj/49.2.M85

48. Bergström, J., Hermansen, L., Hultman, E. \& Saltin, B. Diet, Muscle Glycogen and Physical Performance. Acta Physiol. Scand. (1967). doi:10.1111/j.17481716.1967.tb03720.x

49. Phielix, E. et al. Lower intrinsic ADP-stimulated mitochondrial respiration underlies in vivo mitochondrial dysfunction in muscle of male type 2 diabetic patients. Diabetes (2008). doi:10.2337/db08-0391

50. Boushel, R. et al. Patients with type 2 diabetes have normal mitochondrial function in skeletal muscle. Diabetologia (2007). doi:10.1007/s00125-007-0594-3

51. Hoeks, J. et al. Prolonged fasting identifies skeletal muscle mitochondrial dysfunction as consequence rather than cause of human insulin resistance. Diabetes (2010). doi:10.2337/db10-0519

52. van Moorsel, D. et al. Demonstration of a day-night rhythm in human skeletal muscle oxidative capacity. Mol. Metab. (2016). doi:10.1016/j.molmet.2016.06.012

53. Schrauwen-Hinderling, V. B. et al. Impaired in vivo mitochondrial function but similar intramyocellular lipid content in patients with type 2 diabetes mellitus and BMImatched control subjects. Diabetologia (2007). doi:10.1007/s00125-006-0475-1

54. Kemp, G. J. \& Radda, G. K. Quantitative interpretation of bioenergetic data from 31P and $1 \mathrm{H}$ magnetic resonance spectroscopic studies of skeletal muscle: an analytical review. Magnetic resonance quarterly (1994). 
55. Weir, J. B. d. V. New methods for calculating metabolic rate with special reference to protein metabolism. J. Physiol. (1949). doi:10.1113/jphysiol.1949.sp004363

56. Matomäki, P., Linnamo, V. \& Kyröläinen, H. A Comparison of Methodological Approaches to Measuring Cycling Mechanical Efficiency. Sport. Med. - Open (2019). doi:10.1186/s40798-019-0196-x

57. van der Berg, J. D. et al. Identifying waking time in 24-h accelerometry data in adults using an automated algorithm. J. Sports Sci. (2016). doi:10.1080/02640414.2016.1140908

58. Molenaars, M. et al. A Conserved Mito-Cytosolic Translational Balance Links Two Longevity Pathways. Cell Metab. (2020). doi:10.1016/j.cmet.2020.01.011

59. The R Development Core Team. R: A language and environment for statistical computing. R Foundation for Statistical Computing Vienna, Austria. ISBN 3-90005107-0, URL http://ww (2010).

60. Gentleman, R. C. et al. Bioconductor: open software development for computational biology and bioinformatics. Genome Biol. (2004). doi:10.1186/gb-2004-5-10-r80

61. Wickham, H., François, R., Henry, L. \& Müller, K. dplyr: A Grammar of Data Manipulation. R package version. Media (2019).

62. Rohart, F., Gautier, B., Singh, A. \& Lê Cao, K. A. mixOmics: An R package for 'omics feature selection and multiple data integration. PLoS Comput. Biol. 13, e1005752 (2017).

63. Ritchie, M. E. et al. limma powers differential expression analyses for RNAsequencing and microarray studies. Nucleic Acids Res. 43, e47 (2015).

64. Law, C. W., Chen, Y., Shi, W. \& Smyth, G. K. voom: Precision weights unlock linear model analysis tools for RNA-seq read counts. Genome Biol. 15, R29 (2014).

65. Csardi, G. \& Nepusz, T. The igraph software package for complex network research. InterJournal Complex Sy, 1695 (2006).

66. Wickham, H. Ggplot2. Wiley Interdiscip. Rev. Comput. Stat. 3, 180-185 (2011).

67. Kassambara, A. Package 'ggpubr': 'ggplot2' Based Publication Ready Plots. $R$ Packag. version 0.4.0 (2020).

68. Slowikowski, K. ggrepel: Automatically Position Non-Overlapping Text Labels with 'ggplot2'. R package version 0.8.2 (2020).

69. Neuwirth, E. RColorBrewer: ColorBrewer palettes. $R$ Packag. version 1.1-2 https://cran.R-project.org/package=RColorBrewer (2014). 


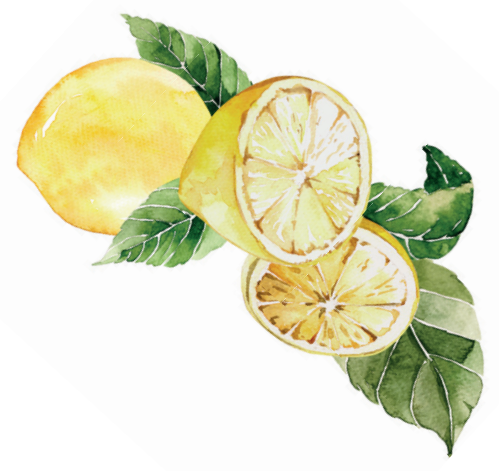

\section{CHAPTER 5}

$\mathrm{NAD}^{+}$- precursor supplementation with L-tryptophan, nicotinic acid, and nicotinamide does not affect mitochondrial function or skeletal muscle function in physically compromised older adults

Niels J. Connell, Lotte Grevendonk, Ciarán E. Fealy, Esther Moonen-Kornips,

Yvonne M.H. Bruls, Vera B. Schrauwen-Hinderling, Johan de Vogel, Robert Hageman, Jan Geurts, Ruben Zapata-Perez, Riekelt H. Houtkooper, Bas Havekes, Joris Hoeks, Patrick Schrauwen

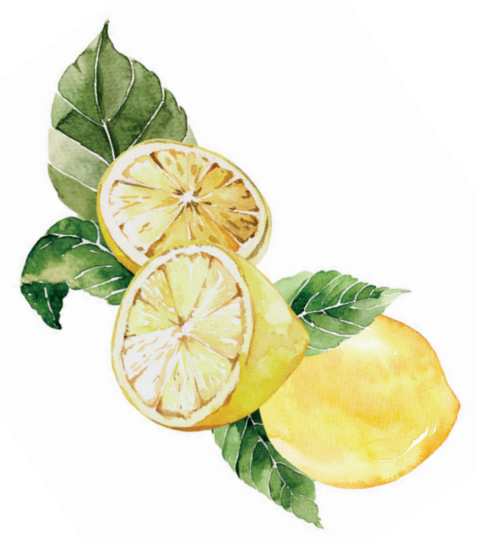




\section{Abbreviations}

${ }^{1} \mathrm{H}-\mathrm{MRS} \quad{ }^{1} \mathrm{H}$-Magnetic resonance spectroscopy

ADP Adenosine diphosphate

ADPr Adenosine diphosphate riboside

AMPK 5' adenosine monophosphate-activated protein kinase

ATP Adenosine triphosphate

BMI Body mass index

BMR Basal metabolic rate

CytC Cytochrome C

DBP Diastolic blood pressure

ETC Electron transport chain

FCCP Carbonylcyanide-4-(trifluoromethoxy)-phenylhydrazone

FFA Free fatty acids

GEE Gross exercise efficiency

INT Intervention

MAP Mean arterial pressure

NA Nicotinic acid

NAAD Nicotinic acid adenine dinucleotide

$\operatorname{NAD}(\mathrm{H}) \quad$ Nicotinamide adenine dinucleotide

$\operatorname{NADP}(\mathrm{H}) \quad$ Nicotinamide adenine dinucleotide phosphate

NAM Nicotinamide

NE Niacin equivalent

NEE Net exercise efficiency

NR Nicotinamide riboside

$\mathrm{PCr} \quad$ Phosphocreatine

QA Quinolinic acid

SBP Systolic blood pressure

SPPB Short physical performance battery

TG Triglycerides

L-Trp L-Tryptophan

RER Respiratory exchange ratio

UCP1 Uncoupling protein 1 


\section{ABSTRACT}

Background: Boosting nicotinamide dinucleotide $\left(\mathrm{NAD}^{+}\right)$via supplementation with niacin equivalents has been proposed as a potential modality capable of promoting healthy aging and negating age-dependent decline of skeletal muscle mass and function.

Objective: We investigated the efficacy the efficacy of NAD+-precursor supplementation (tryptophan, nicotinic acid, and nicotinamide) on skeletal muscle mitochondrial function in physically compromised older adults.

Methods: A randomized, double-blind, controlled trial was conducted in fourteen (female/male: 4/10) community-dwelling, older adults with impaired physical function (age: 72.9 (4.0) y; BMI: $25.2(2.3) \mathrm{kg} / \mathrm{m}^{2}$ ). Participants were supplemented with 207.5 mg niacin equivalents/day (intervention, INT) and a control product (CON) that did not contain niacin equivalents, each for 32 days. The primary outcomes tested were mitochondrial oxidative capacity and exercise efficiency and was analyzed by means of paired student's T-test. Secondary outcomes such as NAD ${ }^{+}$concentrations were analyzed accordingly.

Results: Following supplementation, skeletal muscle NAD ${ }^{+}$concentrations (7.5 (1.9) vs 7.9 (1.6) AU in intervention (INT) vs control (CON)) were not significantly different compared to the control condition, whereas skeletal muscle methyl-nicotinamide (MeNAM) levels were significantly higher under $\mathrm{NAD}^{+}$-precursor supplementation (INT 0.098 (0.063) vs CON 0.025 (0.014); $p=0.001)$, suggesting increased NAD ${ }^{+}$ metabolism. Conversely, both ADP-stimulated (INT 82.1 (19.0) vs CON 84.0 (19.2); $\mathrm{p}=0.716$ ) and maximally uncoupled mitochondrial respiration (INT 103.4 (30.7) vs CON 108.7 (33.4); $\mathrm{p}=0.495)$ did not improve under $\mathrm{NAD}^{+}$-precursor supplementation; neither did net exercise efficiency during the submaximal cycling test (INT 20.2 (2.77) vs CON 20.8 (2.88); p = 0.342).

Conclusions: Our findings are consistent with previous findings on NAD ${ }^{+}$efficacy in humans and we show in community-dwelling, older adults with impaired physical function that $\mathrm{NAD}^{+}$-precursor supplementation through L-tryptophan, nicotinic acid, and nicotinamide does not improve mitochondrial or skeletal muscle function. 


\section{INTRODUCTION}

Aging has been defined as the time-dependent decline of function (1), with one of the most striking features of the aging process being the progressive loss of skeletal muscle mass (sarcopenia), and in turn, the decline of skeletal muscle physical function (2). The loss of skeletal muscle mass and function leads to mobility impairments, increased risk of falls, physical frailty, and metabolic impairments (3). Hence, a compromised physical function increases the risk of dependency in activities of daily living and care needs. With society confronted with an ever-aging population, a large effort of research has focused on identifying strategies to promote healthy aging.

Interestingly, the loss of skeletal muscle function is paralleled by an age-dependent decline in mitochondrial function (1), an established hallmark of aging that is considered to be a driving force behind skeletal muscle aging (4-6). Therefore, promoting mitochondrial metabolism may be a promising strategy to negate the metabolic and functional disturbances seen in skeletal muscle during aging (7).

One pathway that has received much attention in this context is the nicotinamide adenine dinucleotide $\left(\mathrm{NAD}^{+}\right.$) - sirtuin (SIRT)-axis (8-11). The SIRT enzyme family is comprised by $\mathrm{NAD}^{+}$-dependent deacetylases involved in the regulation of mitochondrial metabolism (12) and act as sensors to the bioavailability of $\mathrm{NAD}^{+}(13)$. The co-enzyme $\mathrm{NAD}^{+}$and its reduced form NADH are critical to the cellular redox potential and are the predominant electron donor in the electron transport chain (ETC). NAD ${ }^{+}$, however, is the rate-limiting substrate in SIRT activity, and NAD ${ }^{+}$ bioavailability has been shown to decline with increasing age in mice $(4,10)$ and humans $(14,15)$. The human body derives $\mathrm{NAD}^{+}$from various dietary sources, as it can be synthesized de novo from the essential amino-acid L-tryptophan (L-Trp), or from niacin compounds such as nicotinic acid (NA) through the Preiss-Handler pathway or salvaged from nicotinamide (NAM) and nicotinamide riboside (NR) (16). From a dietary perspective, NR is a trace element, whereas L-Trp, NA, and NAM are more abundantly available in our diets (17).

Preclinical work in murine models of aging has demonstrated the efficacy of supplementing with $\mathrm{NAD}^{+}$-precursors, such as NR and nicotinamide mononucleotide $(\mathrm{NMN})$, to negate the effects of aging-induced by declining $\operatorname{NAD}^{+}$levels $(4,18)$, 
promote longevity (18), and improve energy metabolism, body weight, and mitochondrial oxidative metabolism (11). Following suit, several human intervention studies have focused on the use of the $\mathrm{NAD}^{+}$-precursor NR to improve cardiovascular health in middle-aged to older adults (19), to combat metabolic dysfunction in middle-aged obese individuals $(20,21)$, and to improve skeletal muscle metabolism in older adults (22). Although these studies provide some evidence on the efficacy of supplementation with $\mathrm{NAD}^{+}$-precursors in older adults to increase $\mathrm{NAD}^{+}$levels in blood and skeletal muscle through NR supplementation, evidence stemming from studies involving older adults with a compromised physical function and focusing on skeletal muscle function are still lacking.

Furthermore, other than through supplementation with high doses of NR or NA, there is only limited evidence discerning $\mathrm{NAD}^{+}$-precursor supplementation at lower doses. Dietary supplements bearing more resemblance of increased dietary intake may also has the potency to increase $\operatorname{NAD}^{+}$bioavailability in humans $(23,24)$. Moreover, increasing $\mathrm{NAD}^{+}$synthesis from L-Trp by inhibiting the ACMSD enzyme also leads to activation of SIRT1 and its downstream health benefits (25). Here, we aim to elucidate the efficacy of $\mathrm{NAD}^{+}$-precursor supplementation through a novel combination of L-Trp, NA, and NAM on skeletal muscle mitochondrial function in community-dwelling, older adults with impaired physical function.

\section{METHODS AND MATERIALS}

\section{Participants}

Fourteen physically compromised, older adults (65 years and older, BMI 20 - 30 $\mathrm{kg} / \mathrm{m}^{2}$ ) were recruited from the general population in the vicinity of Maastricht through advertisements. The study was conducted in accordance with the principles of the declaration of Helsinki and approved by the Ethics Committee of the Maastricht University Medical Center+. All participants provided written informed consent and the study was registered at clinicaltrials.gov (NCT03310034).

All participants (4 females, 10 males) were Caucasian, non-smokers, and generally healthy as predetermined by a medical questionnaire and physical examination by a physician, with only 7 participants using prescription medication, with amongst 
others: anti-hypertensives, statins, and proton pump inhibitors (summarized in Table 1). Impaired physical function of the participants was defined as a walking distance of 465 meters or less as assessed during a six-minute walk test (6MWT). Thus, during a clocked 6-minute session, participants were instructed to walk as far as possible without running, over a 20-meter flat surface track. The cut-off used to define impaired physical function was based on the median 6MWT walking distance (personal communication with Prof. Dr. Ir. C.P.G.M. de Groot, Dept. Of Agrotechnology and Food Sciences, Human Nutrition \& Health, Wageningen University and Research) of the pre-frail subgroup of the NU-AGE cohort (26). Habitual physical activity was assessed and analyzed by Baecke's Habitual Physical Activity Questionnaire (27). Exclusion from participation was primarily based on the following: excessive alcohol and/or drug abuse, physical activity > 1 hour/week, inability to complete the required measurements, type 2 diabetes, significant allergies or intolerances concerning the study products, and medication use known to hamper the participant's safety during the study procedures.

The study was conducted between November 2017 and February 2020. Conduction of the study protocol was hampered and curtailed prematurely during the SARSCoV-2 (COVID-19) virus outbreak in the Netherlands in accordance with the imposed governmental restrictions in early 2020. Ultimately, 13 participants completed the study protocol, with the fourteenth participant completing only the first research period and wash-out of the study protocol. 
Table 1 Baseline participant characteristics at screening.

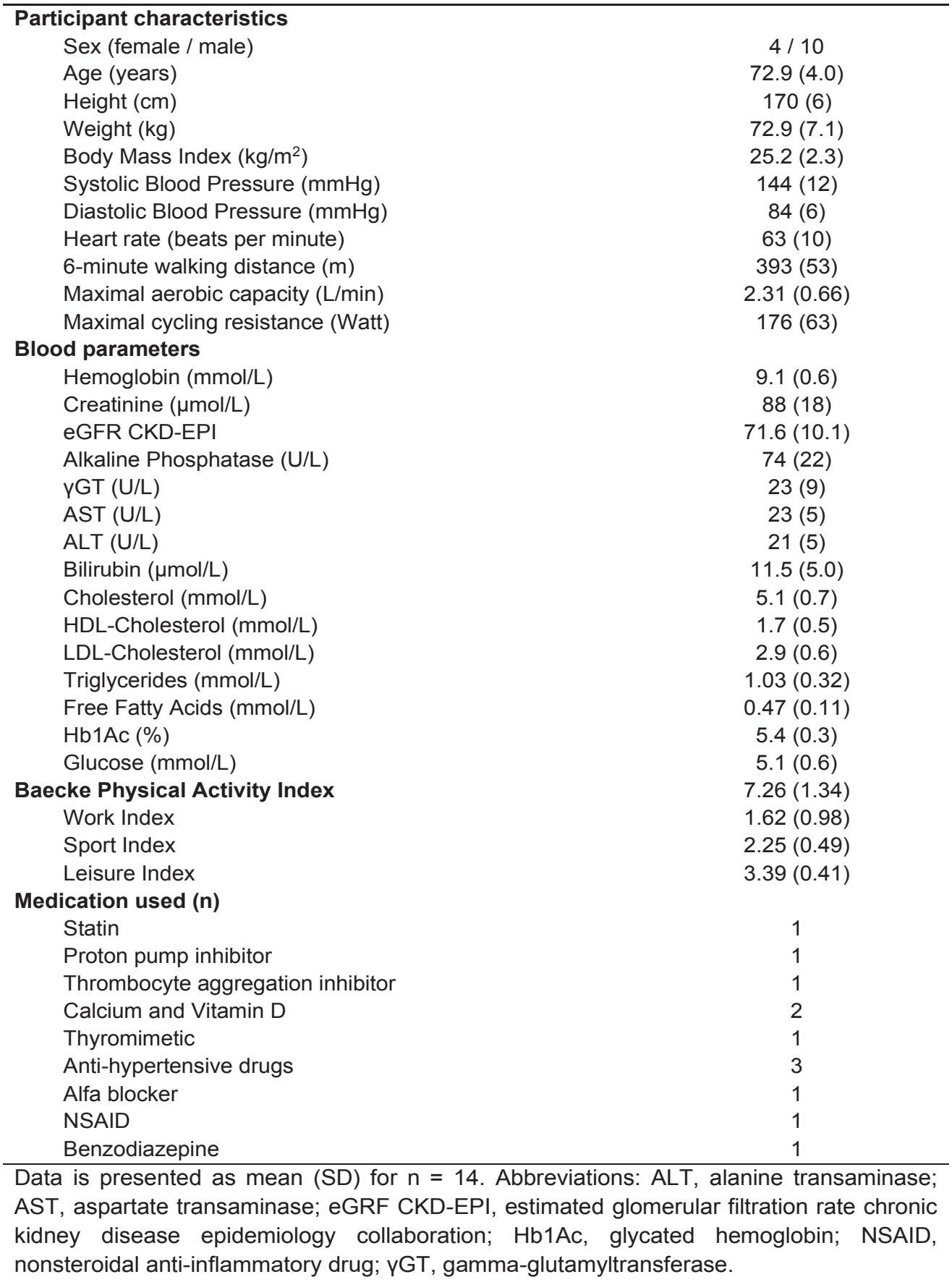




\section{Chapter 5}

\section{Study design}

We conducted a double-blind, randomized, controlled, cross-over trial in 13 physically compromised, older adults. Primary outcome parameters were ex and in vivo mitochondrial function and resting and submaximal exercise energy expenditure. Secondary outcome parameters were glucose tolerance, ectopic fat content, acetylcarnitine levels and physical function. All other outcome measures were exploratory. Each study period consisted of 32 days of supplementation with either the intervention product (INT) or control (CON), separated by at least 5 weeks of wash-out. We chose this intervention duration, based on previous studies in our lab with other nutritional supplements, such as resveratrol, that showed effects on metabolic outcome parameters (28). During the entire study, participants were instructed to maintain habitual physical activity levels and dietary choices and no dietary restrictions were imposed. An overview of the study design is presented in Figure 1. 


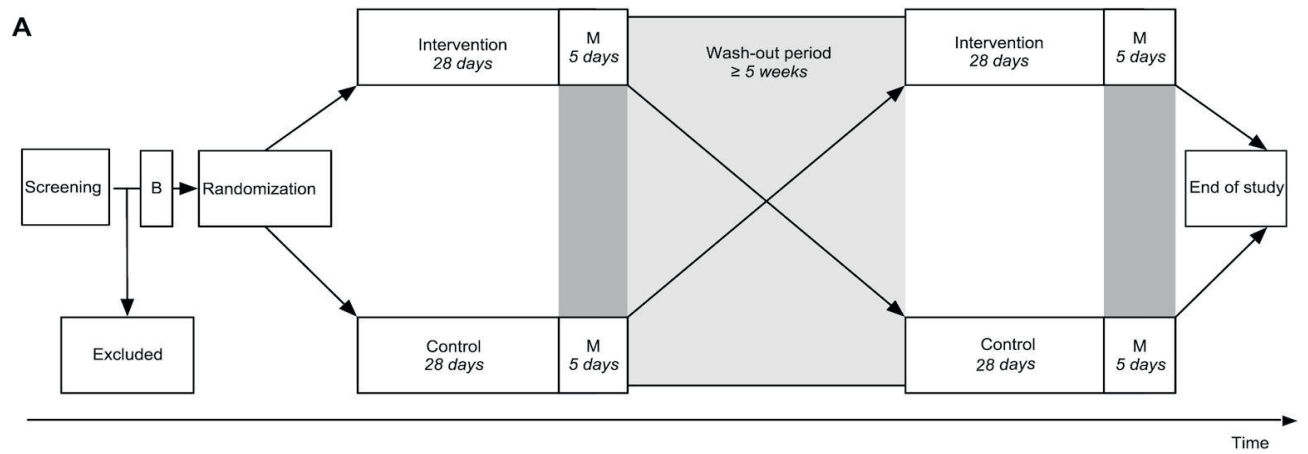

B

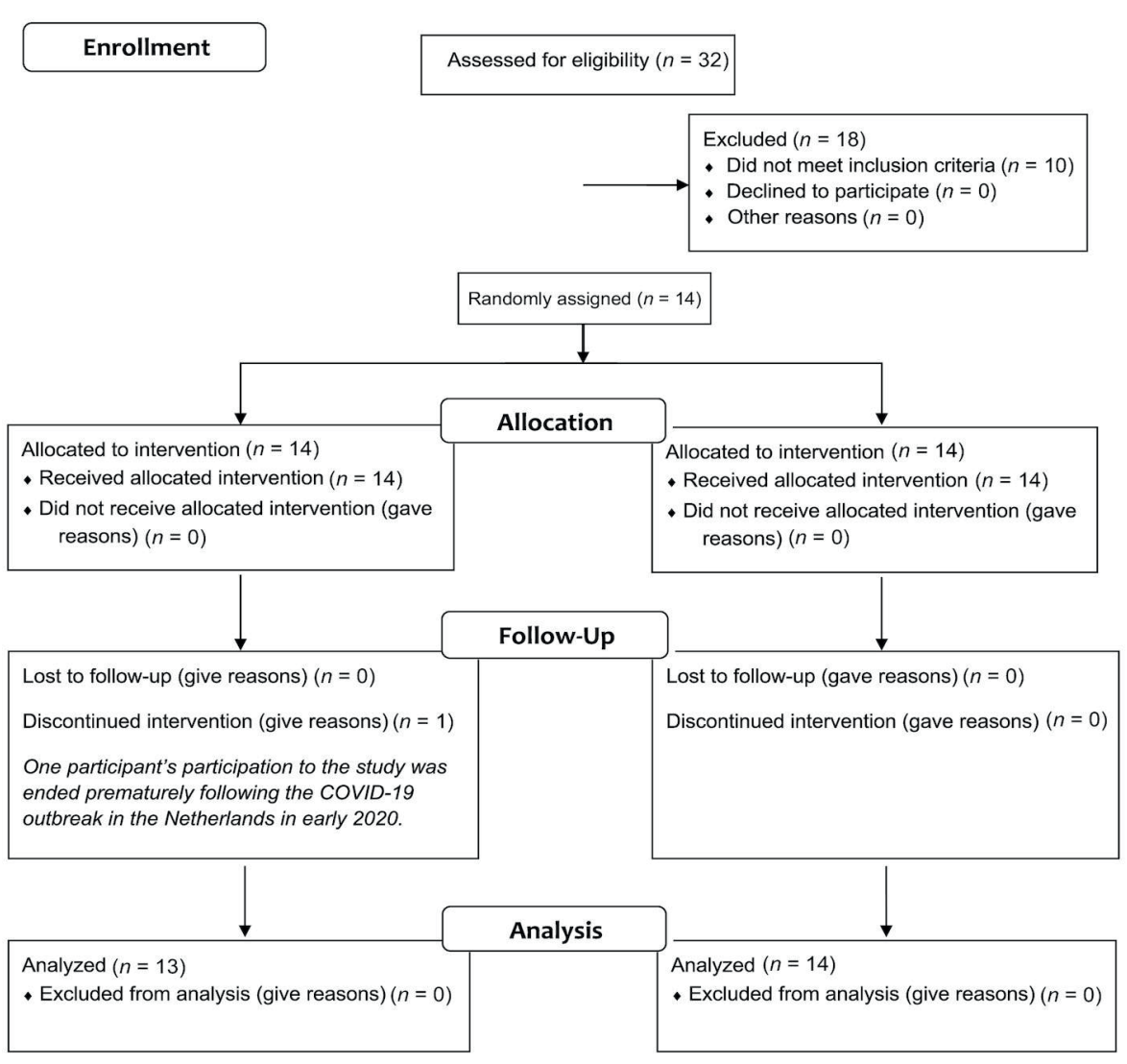

Figure 1 (A) Study timeline and (B) study protocol depicting a CONSORT flow diagram of participant inclusion in the study. Abbreviations: $B$, baseline measurements preceding randomization; COVID-19, coronavirus disease 2019; $\mathrm{M}$, end of study period measurements on days 29,31 , and 33 


\section{Supplementation protocol}

The intervention product (INT) was composed of a whey protein powder as a source of tryptophan, to which NA and NAM were added. In total, the equivalent of 207.5 $\mathrm{mg} /$ day niacin equivalents (NE) of $\mathrm{NAD}^{+}$-precursors was supplemented through LTrp (210 mg/day at a 60:1 conversion rate $(29,30))$, NA (4 mg/day), and NAM (200 $\mathrm{mg} /$ day). The control product ( $\mathrm{CON}$ ) was composed of an amino acid powder mixture resembling whey protein, but lacking L-Trp, NA, and NAM. Participants received the dietary supplements in a single daily serving, to be ingested during breakfast after dissolving the powder in $200 \mathrm{~mL}$ of orange juice. Production and packaging of the supplements were done by Danone Research and approved by the Toxicology and Safety department of Danone Research.

\section{Measurements}

Baseline testing

At baseline, participants underwent a maximal cycling test determining maximal aerobic capacity $\left(\mathrm{VO}_{2} \mathrm{max}\right)$ and maximal power output (Wmax) on an electronically braked cycle ergometer (Lode Excalibur, Groningen, The Netherlands), as described previously (31). $\mathrm{O}_{2}$ consumption and $\mathrm{CO}_{2}$ production were measured using indirect calorimetry (Omnical, IDEE, Maastricht, the Netherlands), and the respiratory exchange ratio (RER) was monitored as a verification of the participant's maximal effort.

\section{Day 1 - Start of supplementation}

Following the baseline cycling test, participants were randomly allocated to the intervention or control arm and supplementation was initiated. Participants then reported to our facility on Day 1 and 15 to measure blood pressure, to provide a venous blood sample, and to fill out the RAND-36 Health Survey 1.0 questionnaire $(32,33)$. The venous blood sample was analyzed for standard clinical lab values in the Maastricht Hospital. The participants were randomized in blocks of 4 using the website Randomizer.org for randomization by an independent researcher.

Blood pressure was measured in sitting position on the non-dominant arm with an oscillometric cuff (Omron M6 Comfort, OMRON Healthcare Europe B.V., Hoofddorp, the Netherlands) in triplicate from which mean arterial pressure (MAP) was estimated from the systolic blood pressure (SBP) and diastolic blood pressure (DBP) 
mean values of the measurement and calculated as MAP $=[(2 / 3 \times \mathrm{DBP})+(1 / 3 \times$ SBP)].

\section{Day 29 - Hepatic lipid content}

On day 29, participants reported to our facility in the fasted state at 7 AM to undergo proton magnetic resonance spectroscopy $\left({ }^{1} \mathrm{H}-\mathrm{MRS}\right)$ for the quantification of hepatic lipid content (intrahepatic lipid, IHL) with a 3T whole-body MR system (Achieva 3Tx; Philips Healthcare, Best, the Netherlands), as described previously (34). To prevent motion artifacts, participants were asked to breathe in the rhythm of the spectroscopic measurement. Subsequently, the water signal dominating the proton spectra was suppressed using frequency-selective pre-pulses and the spectra was fitted to quantify the lipid peak. The lipid/water ratio was used as a parameter of intrahepatic lipid content, as previously reported (34).

Next, a venous blood sample was acquired, the RAND-36 Health Survey 1.0 questionnaire was repeated and the short physical performance battery (SPPB) (35) was performed to assess physical function of the lower extremities. Lastly, blood pressure was measured as described previously under Day 1.

\section{Day 31 - Muscle volume}

The participants underwent another MRI session at 5 PM on Day 31 to determine the muscle volume of the upper leg. Henceforth, the participant was positioned in the 3T MRI scanner (Achieva 3Tx; Philips Healthcare, Best, the Netherlands) in the supine position, feet first. T2 weighted MRI measurements were acquired covering the from the patella to the art. coxae, allowing muscle volume to be determined using $3 \mathrm{D}$ segmentation.

\section{Day 33 - Submaximal cycling test and skeletal muscle biopsy}

On the last day of the study period (Day 33), participants reported to the research facility in the fasted state (from 10 PM the evening before). Body composition was measured by means of air displacement plethysmography (BodPod $®$, COSMED, Rome, Italy). Subsequently, indirect calorimetry through a ventilated hood system was used to determine the basal metabolic rate (BMR) and respiratory exchange ratio (RER) over a period of 30 minutes. 
Next, a skeletal muscle biopsy was obtained from the $m$. vastus lateralis according to the Bergström method (36) with a side-cutting needle under local anesthesia using $1.0 \%$ lidocaine $(10 \mathrm{mg} / \mathrm{mL})$ without epinephrine, from which a portion was kept in a preservation medium and freshly used to prepare permeabilized muscle fibers according to Hoeks et al. (37). Subsequently, ex vivo high-resolution respirometry was performed using a two-chamber Oxygraph (OROBOROS Instruments, Innsbruck, Austria) using a multiple substrate/inhibitor titration protocol to extensively characterize the mitochondrial capacity of skeletal muscle tissue, as previously reported (38). Measurements were performed in quadruplicate and the integrity of the outer mitochondrial membrane was assessed by the addition of cytochrome $\mathrm{c}$ (CytC) upon maximal coupled respiration (State 3) and deemed intact if the response to the CytC addition remained under 10\%, hence 16 out of 100 individual measurements were excluded.

Following the muscle biopsy, participants were submitted to a submaximal exercise session of one hour on an electrically braked cycling ergometer (Lode Excalibur, Groningen, The Netherlands) at $50 \%$ of their Wmax achieved in the maximal cycling test at baseline. indirect calorimetry was measured during the second and fourth quarter of the one-hour session.

\section{Calculations}

From the indirect calorimetry data, energy expenditure and substrate oxidation rates were calculated using Weir's formula under the assumption of negligible protein oxidation (39). In addition, gross exercise efficiency (GEE) was calculated as [Wext $/$ $E_{\text {tot }}$ and net exercise efficiency (NEE) as [Wext / (Etot - Erest)] (40) from the submaximal exercise indirect calorimetry data.

\section{Biochemical analyses}

\section{Plasma metabolites}

Using EDTA plasma, glucose (Horiba, Montpellier, France), insulin (Crystal Chem, Elk Grove Village, Illinois, USA), total free fatty acids (FFA) (Wako, Neuss, Germany), and triglycerides (TG) (Sigma, Saint Louis, Missouri, USA) concentrations were determined in samples collected on Day 1 and 29 by means enzymatic quantification with the ABX Pentra C400 (Horiba, Montpellier, France). All time points 
of one participant were measured in the same run with insulin and FFA levels measured in duplicates.

\section{Acylcarnitine analysis}

2-4 mg of freeze-dried muscle tissue was used to analyze skeletal muscle acylcarnitines, as previously described (41) . Quantitative determination of the propylated acylcarnitines in the medium was performed using tandem mass spectrometry in an Acquity UPLC System (Waters) coupled to a Quattro Premier XE Tandem Quadrupole Mass Spectrometer (Waters). The internal standards d3-0, d33, d3-6, d3-8, d3-10 and d3-16 carnitine were purchased from Dr. Herman J. ten Brink (Vrije Universiteit Medical Hospital, Amsterdam, The Netherlands).

\section{Enzymatic cyclic assay for quantitative NAD determination}

1-5 $\mathrm{mg}$ of freeze-dried muscle tissue were homogenized in $250 \mu \mathrm{L}$ of $2 \mathrm{M} \mathrm{HClO}_{4}$ using a TissueLyser II (Qiagen) bead mill for 5 minutes at 30 pulses per second. After centrifugation at $16000 \mathrm{~g}, 100 \mu \mathrm{L}$ of the acidic supernatant were neutralized by addition of $150 \mu \mathrm{L} 2 \mathrm{M} / 0.6 \mathrm{M} \mathrm{KOH} / \mathrm{MOPS}$ and centrifuged again to remove precipitated salts. $\mathrm{NAD}^{+}$content was determined using an enzymatic spectrophotometric cycling assay based on the coupled reaction of malate and alcohol dehydrogenases, as previously described (42). Enzymes used for $\mathrm{NAD}^{+}$ determination (alcohol dehydrogenase from yeast, malate dehydrogenase from pig heart, and glutamate oxaloacetic transaminase from pig liver) were purchased from Sigma.

\section{Metabolomics procedure}

2-4 mg of freeze-dried muscle tissue was used for metabolomics analysis as previously described (20). Briefly, metabolite analysis was carried out in an Aquity UPLC system (Waters) coupled to an Impact IITM Ultra-High Resolution Qq-Time-OfFlight mass spectrometer (Bruker). Chromatographic separation of the compounds was achieved using a SeQuant ZIC-cHILIC column (PEEK $100 \times 2.1 \mathrm{~mm}, 3 \mu \mathrm{m}$ particle size, Merck, Darmstadt, Germany) at $30{ }^{\circ} \mathrm{C}$. The LC method consisted in a gradient running at $0.25 \mathrm{~mL} / \mathrm{min}$ from $100 \%$ mobile phase $\mathrm{B}(9: 1$ acetonitrile:water with $5 \mathrm{mM}$ ammonium acetate $\mathrm{pH}$ 6.8) to $100 \%$ mobile phase $\mathrm{A}$ (1:9 acetonitrile:water with $5 \mathrm{mM}$ ammonium acetate $\mathrm{pH} 6.8$ ) in 28 minutes, followed by 
are-equilibration step at $100 \%$ B of 5 minutes. MS data were acquired both in negative and positive ionization modes in full scan mode over the range of $\mathrm{m} / \mathrm{z} 50$ 1200.

\section{Statistical analyses}

The variation in the primary outcome mitochondrial state 3 respiration on a combination of malate, octanoyl-carnitine, glutamate and succinate $\left(\sigma=\sim 8 \mathrm{pmol}^{*} \mathrm{mg}\right.$ wet tissue ${ }^{-1 *} \min ^{-1}$ ) was established previously during a pre- and post-intervention high-resolution respirometry (43). We have previously shown that the difference in mitochondrial function between healthy and insulin resistance volunteers is $\sim 20 \%$. To detect a difference of $\sim 10 \%$ increase in mitochondrial function $\left(\mu=\sim 6.0\right.$ pmol $^{*} \mathrm{mg}$ wet tissue ${ }^{-1 *} \min ^{-1}$ ), which we believe is physiologically relevant in this population and which is achievable with a short-term nutritional intervention as demonstrated previously (28), 14 participants must be included with a power ( $\pi$ ) of $80 \%$ and a twosided significance level $(\alpha)$ of 0.05 .

Statistical analysis was performed in SPSS 26 for Mac OS (IBM $®$ SPSS $®$ Statistics, USA) and graphs were made in Prism 8.4.2 for Windows (GraphPad Software, Inc., USA). Descriptive statistics were reported for all data parameters as mean \pm standard deviation (SD), unless otherwise stated. Normality of the data distributions were evaluated with the Kolmogorov-Smirnov normality test and deemed significant when $p<0.05$. The paired Students t-test was used to compare the results of the intervention to the control, or the equivalent Wilcoxon signed-rank test was used if normality was not met. In the event of missing values, the values were not replaced and the participant in question did not contribute to the analysis of that outcome, hence all analyses encompass $n=13$, unless stated otherwise. No carry-over or order effect was detected. In addition, Pearson correlation coefficients were computed. Bonferroni corrections were imposed to correct for multiple testing of single outcome measures. Moreover, due to multiplicity of the primary outcomes the type 1 error was corrected for using a Bonferroni correction as (alpha / n). Statistical significance was achieved if the $p$-value $<0.05$. 


\section{RESULTS}

$\mathrm{NAD}^{+}$-precursor supplementation increased methyl-nicotinamide but not NAD ${ }^{+}$ bioavailability in skeletal muscle

Compliance to the supplement use was estimated by the fraction of sachets returned compared to the number of sachets provided to the participants. For the supplementation with precursors (INT), 100\% (100\%) of all sachets were consumed, compared to $99 \%$ (range, min-max: 97-100\%) in the control condition (CON). Overall, the products were well tolerated, with only one participant reporting cold sores (herpes labialis), which resolved without intervention. Subject characteristics at the start of the study are summarized in Table 1. No carry-over or order effect was detected for any of the primary outcome parameters.

In order to determine the efficacy of the $\mathrm{NAD}^{+}$-precursor supplementation, the concentration of $\mathrm{NAD}^{+}$as well as several metabolites of the $\mathrm{NAD}^{+}$synthesis pathways were measured in skeletal muscle tissue, obtained from the $m$. vastus lateralis. These analyses showed that $\mathrm{NAD}^{+}$levels were similar between INT and $\operatorname{CON}(p=0.123$, Figure 2).

From the de novo biosynthesis pathway, L-Trp, kynurenine, and quinolinic acid (QA) did not differ between INT and CON ( $p \geq 0.05, n=11$, Figure 2). Synthesis of NAD ${ }^{+}$ from nicotinic acid (NA) through the Preiss-Handler pathway leads to synthesis of nicotinic acid adenine dinucleotide (NAAD), which was not different between NAD and CON ( $p=0.262, n=11$, Figure 2). NAD ${ }^{+}$can also be salvaged from NMN, NAM, and NR, but also in these precursors no differences between INT and CON were observed ( $p \geq 0.05, n=11$, Figure 2 ). Consumption of NAD yields NAM and ADPr, with no differences observed in ADPr levels between INT and CON $(p=0.413, n=$ 11, Figure 2). Conversely, the concentration of the primary breakdown product of NAM, methyl-NAM (MeNAM), which is also commonly described as a marker for increased NAD turnover (22), was measured and revealed significantly higher MeNAM concentrations in INT compared to CON ( $p=0.001$, Figure 2). Alternatively, $\mathrm{NAD}^{+}$can be phosphorylated into nicotinamide dinucleotide phosphate (NADP ${ }^{+}$), in which we did not observe a difference between conditions $(p=0.833, n=11$, Figure 2). The respective reduced forms, NADH and $\mathrm{NADPH}$, also did not differ between conditions ( $p=0.389, p=0.365$ respectively, $n=11$, Figure 2 ). 


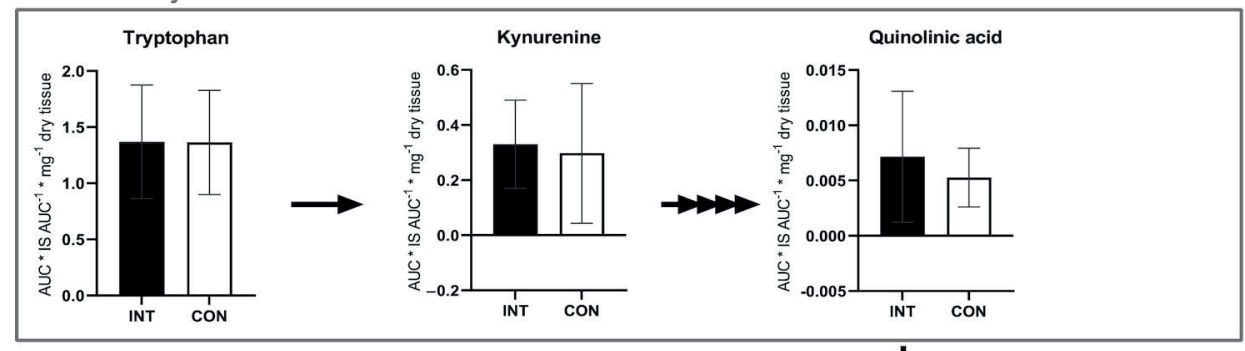

Salvage pathway

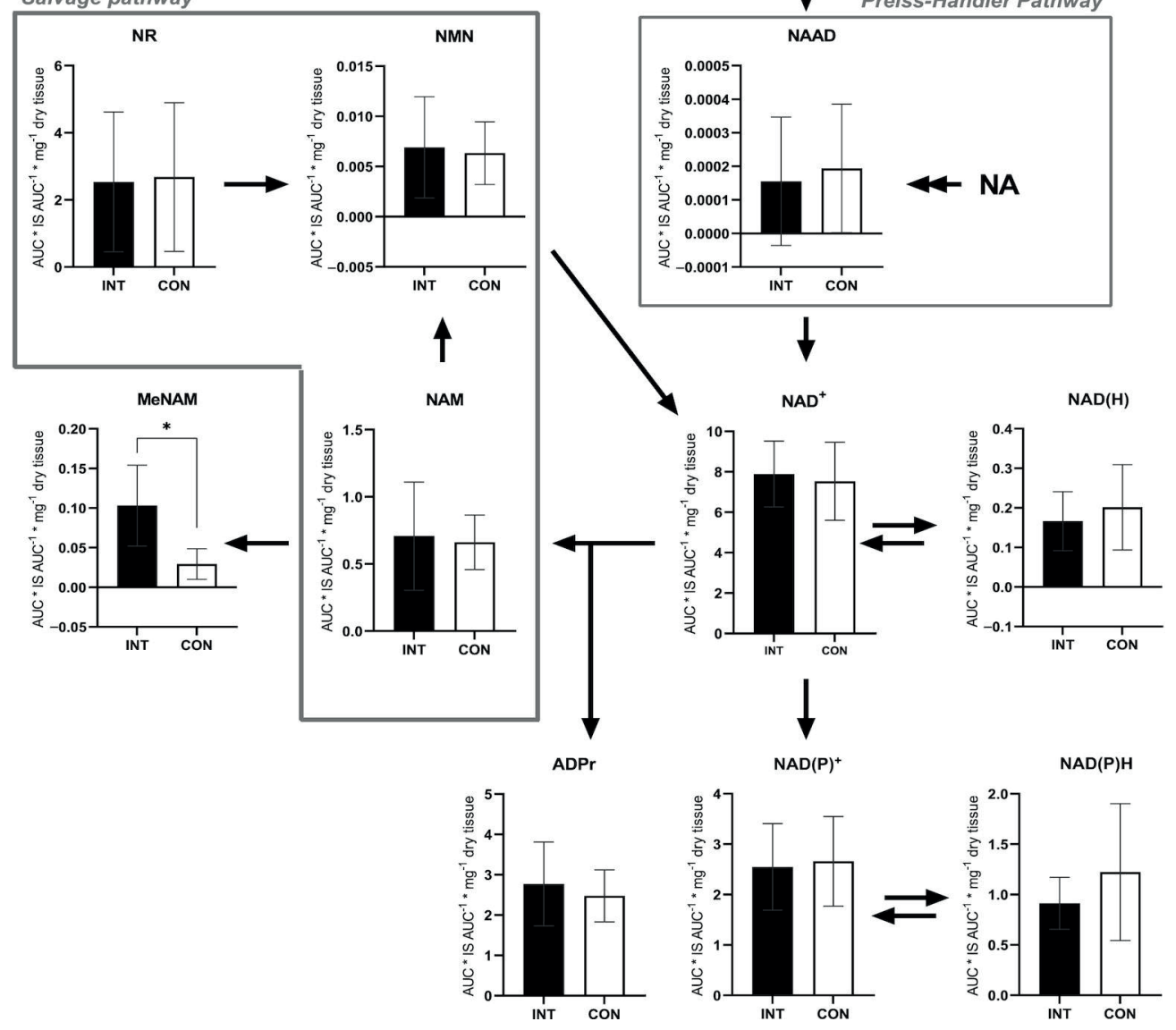

Figure $2 \mathrm{NAD}^{+}$metabolome in skeletal muscle tissue obtained from older adults with compromised physical function administered supplemental niacin equivalents and placebo, each for 32 days. The $y$-axis defines the AUC ( $A \cup C^{*} I S A U C^{-1}$ ) per $\mathrm{mg}$ of dry tissue used in the MS analysis. Data are displayed as means \pm SDs, $n=11$. ${ }^{*} P<0.01$. Abbreviations: ADPr, adenosine diphosphate riboside; AUC, area under curve; CON, control; INT, NAD intervention; IS, internal standard; MeNAM, methyl-nicotinamide; NA, nicotinic acid; NAAD, nicotinic acid adenine dinucleotide; NAM, nicotinamide; NMN, nicotinamide mononucleotide; NR, nicotinamide riboside. 


\section{Skeletal muscle acylcarnitine levels were unaffected by $\mathrm{NAD}^{+}$-precursor supplementation}

We previously showed that NR supplementation in humans altered acylcarnitine levels in skeletal muscle (20). Therefore, acylcarnitine levels were determined in the skeletal muscle tissue and species were categorized in subgroups, being free (C0), independent (C2), short-chain (C3-C5), medium-chain (C6-C12), and long-chain (C13-C20) acylcarnitines. Ultimately, no differences were observed in the concentrations of acylcarnitine subgroups between INT and CON ( $p \geq 0.05$, Figure $3 A B C D, n=11)$.

A
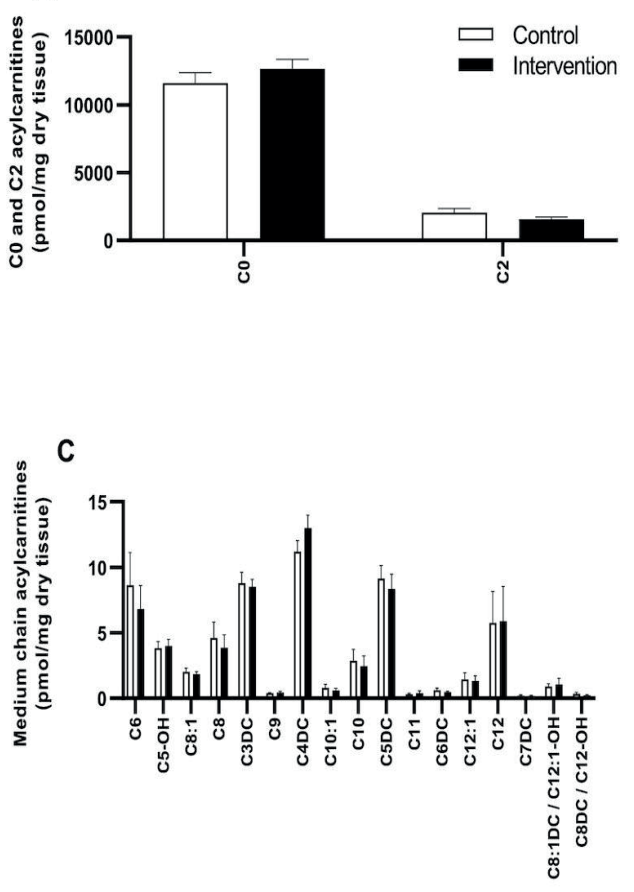

B

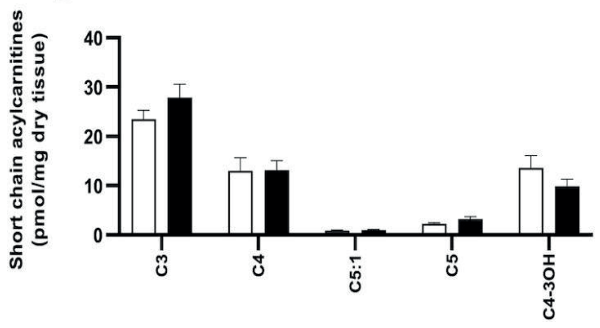

D

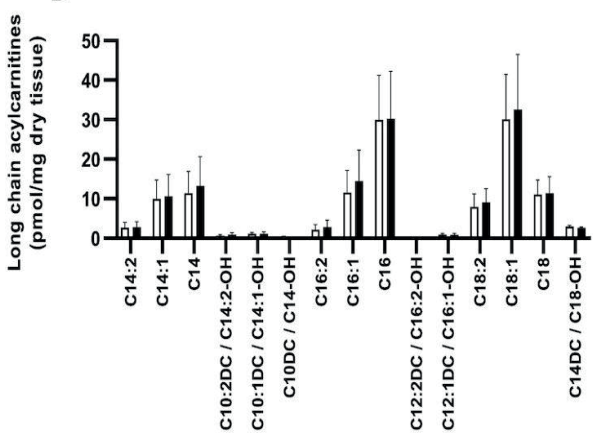

Figure 3 Skeletal muscle acylcarnitine levels in older adults with compromised physical function administered supplemental niacin equivalents and placebo. (A) Acylcarnitine levels in skeletal muscle for free (C0) and independent species (C2). Acylcarnitine levels for (B) short chain, (C) medium chain, and (D) long chain species (LC). Data are displayed as means \pm SDs, $n=11$ 


\section{Body composition was not affected by $\mathrm{NAD}^{+}$-precursor supplementation}

Body weight did not differ between INT $(73.0 \mathrm{~kg}(68.6,77.5)$ and CON $(72.9 \mathrm{~kg}$ (68.6, 77.2), $\mathrm{p}=0.812$ ). Also, body composition was unaffected by $\mathrm{NAD}^{+}$-precursor supplementation as fat percentage was not different for INT and CON (34.2\% (29.3, $39.2)$ and $33.3 \%(27.6,38.9)$ respectively, $p=0.121)$.

\section{$\mathrm{NAD}^{+}$-precursor supplementation did not improve overall ex vivo mitochondrial respiration capacity}

To determine skeletal muscle mitochondrial capacity, we performed ex vivo highresolution respirometry on permeabilized muscle fibers obtained from skeletal muscle biopsies. In the presence of substrates alone (State 2, Figure 4A), oxygen flux was similar in INT and CON, although a slight but significant difference was observed upon malate + glutamate (MG, $p=0.010$ ) between INT and CON. Also, the assessment of ADP-stimulated (State 3 ) respiration fueled by complex I-linked substrates (MO: Figure 4B, $p=0.882$ ) did not reveal any differences between INT and CON. If anything, state 3 respiration upon malate + glutamate tended to be lower in INT vs. CON (MG: $p=0.054$, Figure $4 C$ ). State 3 respiration upon parallel electron input into complex I and II showed no differences between INT and CON with the substrate combinations malate + octanoyl-carnitine + glutamate (MOG, $p=$ 0.753), malate + octanoyl-carnitine + glutamate + succinate (MOGS, $p=0.716$ ), or malate + glutamate + succinate (MGS, $p=0.388$, Figure 4D). Maximal uncoupled respiration (State $3 \mathrm{U}$ ), a reflection of the maximal capacity of the electron transport chain, did not differ between INT and CON ( $p=0.495$, Figure 4E). Lastly, mitochondrial proton leak (State 40) was assessed through the addition of oligomycin and did not differ between INT and CON ( $p=0.888$, Figure 4F). CytC responses were on average $1.32 \%$ for INT and $0.73 \%$ for CON and did not differ between conditions $(p=0.368)$. 

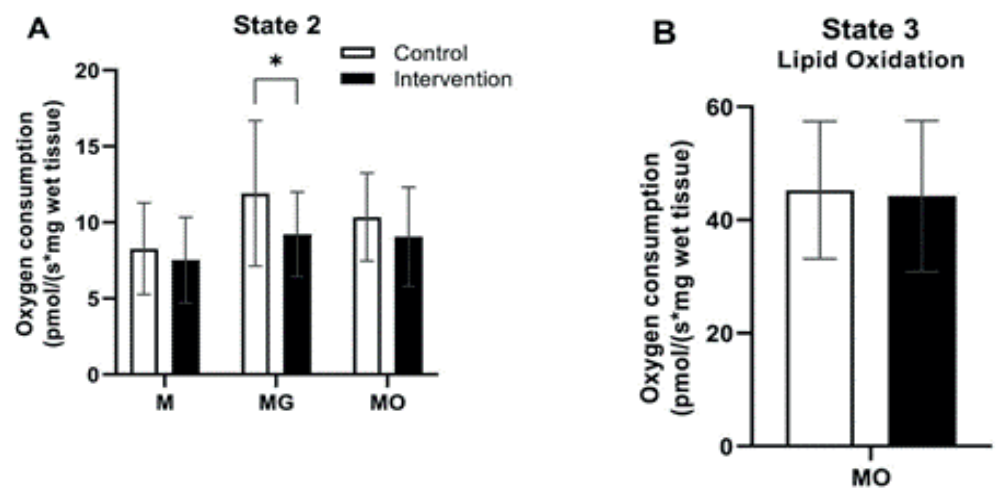
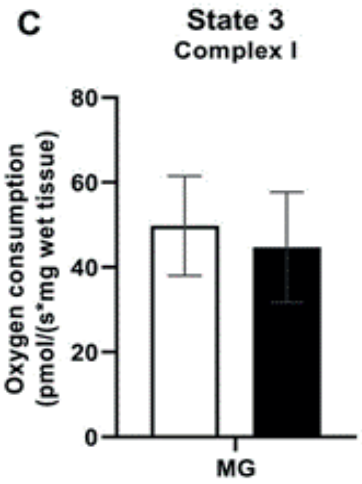

E

$$
\text { State Uncoupled }
$$
Complex I+ II

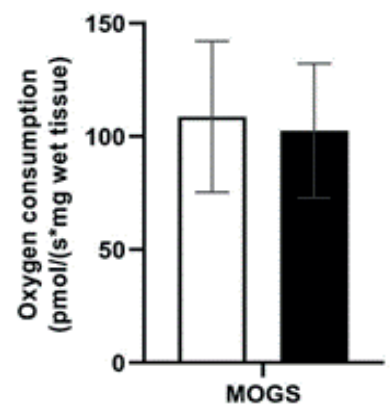

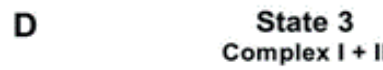

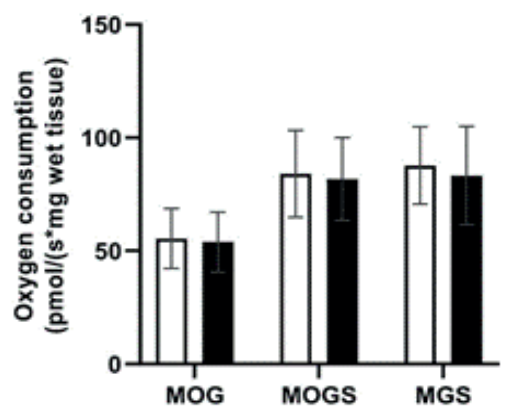

F $\quad \begin{gathered}\text { State } 40 \\ \text { Complex I+ II }\end{gathered}$

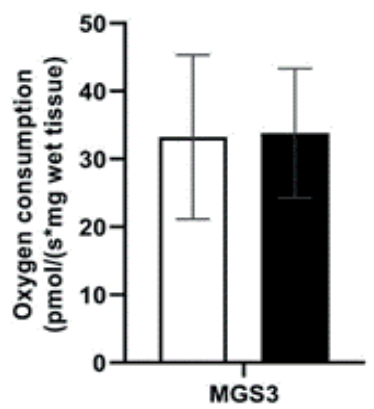

Figure 4 States of mitochondrial respiration of skeletal muscle tissue obtained from older adults with compromised physical function administered supplemental niacin equivalents and placebo, each for 32 days. (A) State 2 respiration. (B) State 3 respiration under ADP stimulation with lipid substrates. (C) State 3 respiration with substrates focusing on Complex I. (D) State 3 respiration with parallel substrates focus on Complex I and II. (E) Uncoupled respiration under FCCP addition. (F) State 40 respiration uncoupled from ATP synthase. Values are represented as means \pm SDs, $n=13$. ${ }^{*} P<0.05$. Abbreviations: FCCP, carbonylcyanide-4-(trifluoromethoxy)-phenylhydrazone; G, glutamate; M, malate; O, octanoyl-carnitine; S, succinate. 


\section{$\mathrm{NAD}^{+}$-precursor supplementation did not affect plasma substrate concentrations}

Plasma samples obtained on Day 29 of each research period were analyzed for the concentrations of glucose, insulin, FFA, and TG. Glucose concentrations did not differ for INT $(5.87 \mathrm{mmol} / \mathrm{L}(5.57,6.16)$ and $\operatorname{CON}(5.76 \mathrm{mmol} / \mathrm{L}(5.52,6.01), \mathrm{p}=$ 0.223). Correspondingly, insulin levels were not different between INT $(9.41 \mathrm{mU} / \mathrm{L}$ $(4.18,14.7)$ and $\operatorname{CON}(9.12 \mathrm{mU} / \mathrm{L}(5.40,12.8), \mathrm{p}=0.807)$. Concentrations of FFA showed no differences between INT $(496 \mu \mathrm{mol} / \mathrm{L}(397,595)$ and CON $(512 \mu \mathrm{mol} / \mathrm{L}$ (416, 609), $p=0.812)$. Lastly, plasma TG levels were not different between INT $(0.830 \mathrm{mmol} / \mathrm{L}(\mathrm{IQR} 0.675,1.03)$ and $\mathrm{CON}(0.990 \mathrm{mmol} / \mathrm{L}(\mathrm{IQR} 0.610,1.43), \mathrm{p}=$ 0.266).

\section{Basal metabolic rate and exercise efficiency were not altered by NAD+- precursor supplementation}

Basal metabolic rate and fasting RER were similar in INT and CON ( $p=0.824$ and $p$ $=0.297$, respectively, Figure 5A and B). As a result, neither carbohydrate nor lipid oxidation in the fasted state was different between INT and CON ( $p=0.588$, Figure $5 \mathrm{C}$ and $\mathrm{p}=0.787$, Figure 5D, respectively).

Besides assessment in resting conditions, substrate utilization was also determined during a submaximal cycling test. All participants completed the first 30 minutes of the cycling test, however, 6 out of 14 participants could not proceed with the cycling test at the set resistance and either decided to cease testing $(n=2)$ or required the resistance to be reduced $(n=4)$ in order to complete the remaining 30 minutes of the test. Therefore, we only report parameters measured in the first half-hour of the cycling test.

Besides substrate utilization, also gross (GEE) and net (NEE) exercise efficiency were also evaluated during the submaximal cycling test. Total energy expenditure during cycling did not differ between INT and CON ( $p=0.103$, Figure 6E), nor did cycling RER, carbohydrate oxidation, or lipid oxidation rates differ between INT and CON $(p=0.366, p=0.186, p=0.466$ respectively, Figure $6 F-H)$. GEE did not differ between conditions ( $p=0.419$, Figure 6I). NEE (i.e. GEE corrected for the BMR,) was also not different between conditions $(p=0.636$, Figure $6 \mathrm{~J})$. 

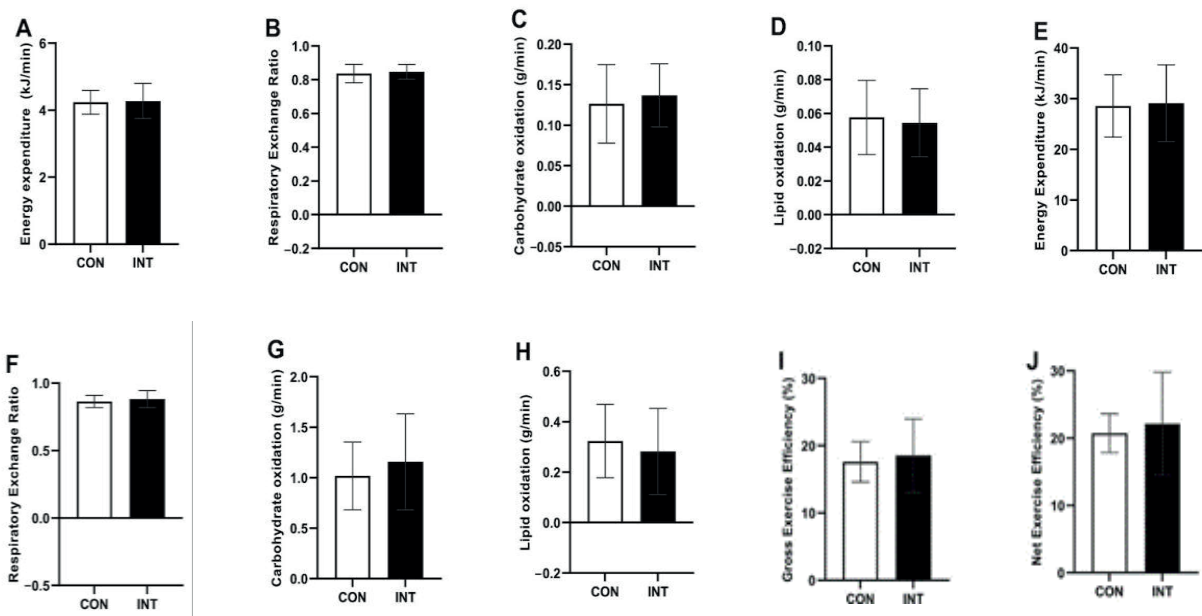

Figure 5 Whole-body indirect calorimetry of older adults with compromised physical function administered supplemental niacin equivalents and placebo. (A) Basal metabolic rate and (B) respiratory exchange ratio, with corresponding (C) carbohydrate oxidation rate and (D) lipid oxidation rate. (E) Submaximal exercise energy expenditure with corresponding (F) respiratory exchange ratio with corresponding $(\mathbf{G})$ carbohydrate and $\mathbf{( H )}$ lipid oxidation rates. (I) Gross and (J) net exercise efficiency from submaximal cycling test. Data are represented as means \pm SDs, $n=13$. Abbreviations: CO, control; INT, NAD intervention.

\section{Intrahepatic lipid content and muscle volume were not affected by NAD+- precursor supplementation}

Intrahepatic lipid content was measured non-invasively by ${ }^{1} \mathrm{H}-\mathrm{MRS}$ and did not differ between INT $(2.8 \%(0.8,4.8)$ and CON $(2.7 \%(1.1,4.3), p=0.624)$. Muscle volume of the $m$. vastus lateralis, assessed by MRI, was not different for INT $\left(1700 \mathrm{~cm}^{3}\right.$ $(1440,1960)$ compared to $\operatorname{CON}\left(1710 \mathrm{~cm}^{3}(1450,1980), \mathrm{n}=11, \mathrm{p}=0.450\right)$.

\section{$\mathrm{NAD}^{+}$-precursor supplementation did not affect blood pressure}

SBP and DBP were measured throughout each study period to evaluate the effect of $\mathrm{NAD}^{+}$-precursor supplementation on this parameter. Summary of the data is presented in Table 2. No differences were observed between INT and CON in SBP on Day 1 ( $p=0.821)$, Day $15(p=0.741)$, and Day $29(n=12, p=0.877)$. DBP did not differ between conditions on Day 1 ( $p=0.551)$, Day 15 ( $p=0.733)$, and Day 29 ( $n=12, p=0.433)$. Lastly, MAP was estimated from the acquired SBP and DBP values and was not different for INT and CON on Day $1(p=0.653)$, Day $15(p=$ 0.976), and Day $29(n=12, p=0.738)$. 
Table 2 Blood pressure in older adults with compromised physical function administered supplemental niacin equivalents and placebo, each for 32 days.

\begin{tabular}{|c|c|c|c|}
\hline & Intervention & Control & P-value \\
\hline \multicolumn{4}{|l|}{$\begin{array}{c}\text { SBP } \\
(\mathrm{mmHg})\end{array}$} \\
\hline Day 1 & $\begin{array}{c}140 \\
(131,148)\end{array}$ & $\begin{array}{c}141 \\
(131,151)\end{array}$ & 0.821 \\
\hline $\begin{array}{c}\text { Day } \\
15\end{array}$ & $\begin{array}{c}140 \\
(131,149)\end{array}$ & $\begin{array}{c}139 \\
(129,148)\end{array}$ & 0.741 \\
\hline $\begin{array}{c}\text { Day } \\
29\end{array}$ & $\begin{array}{c}136 \\
(128,145)\end{array}$ & $\begin{array}{c}137 \\
(130,143)\end{array}$ & 0.877 \\
\hline \multicolumn{4}{|l|}{$\begin{array}{c}\text { DBP } \\
(\mathrm{mmHg})\end{array}$} \\
\hline Day 1 & $\begin{array}{c}82.9 \\
(77.6,88.2)\end{array}$ & $\begin{array}{c}84.3 \\
(80.0,88.6)\end{array}$ & 0.551 \\
\hline $\begin{array}{l}\text { Day } \\
15\end{array}$ & $\begin{array}{c}82.9 \\
(78.1,87.8)\end{array}$ & $\begin{array}{c}83.8 \\
(78.5,89.0)\end{array}$ & 0.733 \\
\hline $\begin{array}{l}\text { Day } \\
29\end{array}$ & $\begin{array}{c}81.1 \\
(75.1,87.0)\end{array}$ & $\begin{array}{c}79.4 \\
(74.8,84.1)\end{array}$ & 0.433 \\
\hline $\begin{array}{c}\text { MAP } \\
(\mathrm{mmHg})\end{array}$ & & & \\
\hline Day 1 & $\begin{array}{c}102 \\
(97,107)\end{array}$ & $\begin{array}{c}103 \\
(97,109)\end{array}$ & 0.653 \\
\hline $\begin{array}{c}\text { Day } \\
15\end{array}$ & $\begin{array}{c}102 \\
(97,107)\end{array}$ & $\begin{array}{c}102 \\
(96,108)\end{array}$ & 0.976 \\
\hline $\begin{array}{l}\text { Day } \\
29\end{array}$ & $\begin{array}{c}99.4 \\
(93.5,105)\end{array}$ & $\begin{array}{c}98.5 \\
(94.0,103)\end{array}$ & 0.738 \\
\hline
\end{tabular}

Values are presented as means (95\% Cls), $n=13$ or 12 (Day 29).

Abbreviations: DBP, diastolic blood pressure; MAP, mean arterial pressure; SBP, systolic blood pressure. 


\section{SPPB performance did not improve under $\mathrm{NAD}^{+}$-precursor supplementation}

The SPPB was used as a clinical measure of physical functioning. Scores for the balance test, the walking speed, and the chair-rise test were similar in INT and CON ( $p>0.999, p=0.500$, and $p=0.489$, respectively). The overall score on the SPPB was also not different between INT (11.0 (IQR 10.00, 11.5) and CON (11.0 (IQR $9.50,12.0), p>0.999)$.

\section{Perceived physical functioning was positively influenced by $\mathrm{NAD}^{+}$-precursor supplementation}

The RAND-36 Health Survey 1.0 questionnaire was used as a measure of perceived health status. Although measured physical functioning was unaffected by NAD+precursor supplementation, significantly higher scores were observed for the perceived physical functioning domain in INT (92.0 (IQR 90.0, 100) as compared to $\operatorname{CON}(90.0$ (IQR 86.3, 93.8), $n=12, p=0.031$, Figure 6). However, the other 8 out of 9 domains of the RAND-36 did not show any differences between INT and CON on Day $29(p \geq 0.05$, Figure 6$)$.

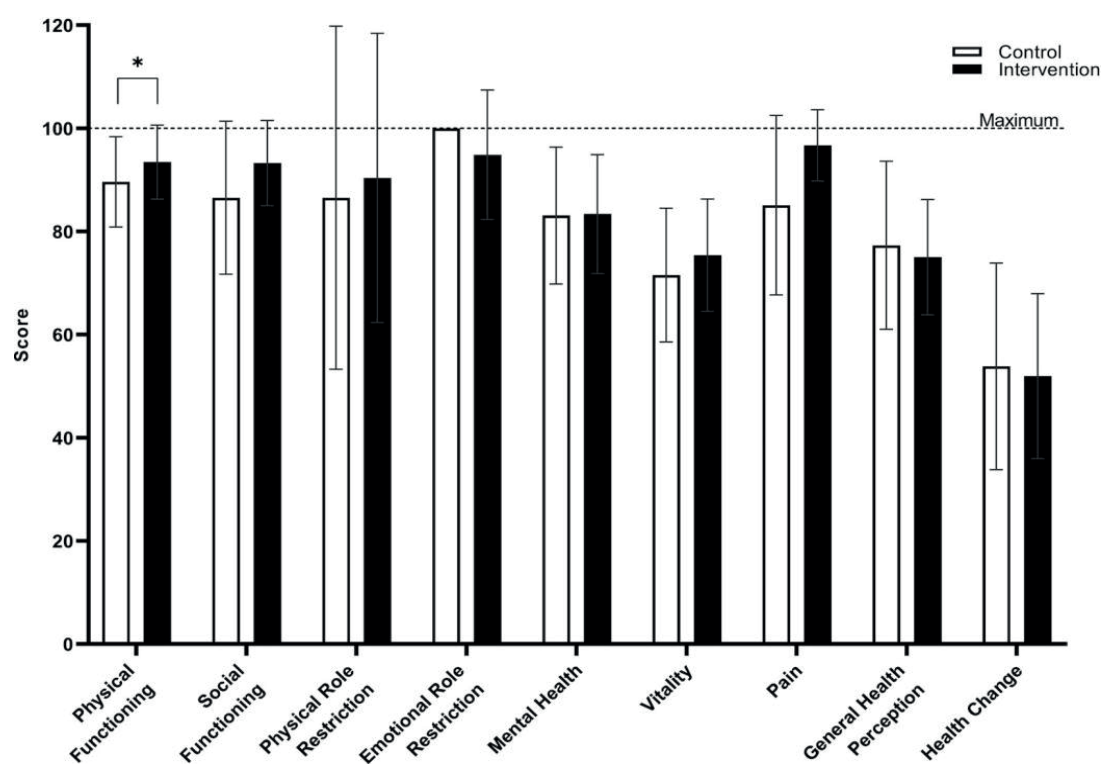

Figure 6 RAND-36 Health Survey 1.0 questionnaire of older adults with compromised physical function administered supplemental niacin equivalents and placebo over 8 domains on Day 29. All domains can be scored between 0 and 100, with higher scores being better. Data are represented as means $\pm \mathrm{SDs}, n=12,{ }^{*} P<0.05$. 


\section{DISCUSSION}

Mitochondrial dysfunction is an established hallmark of aging and together with declining $\mathrm{NAD}^{+}$levels, these factors are thought to drive the age-dependent decline in skeletal muscle physical function. Here, we hypothesized that supplementation with the $\mathrm{NAD}^{+}$-precursors L-Trp, NA, and NAM, in a dose that could be achieved through increased dietary intake, would improve mitochondrial oxidative capacity in community-dwelling, older adults with impaired physical function. We tested this hypothesis by conducting a double-blind, randomized, controlled, cross-over trial with detailed metabolic phenotyping. We show that supplementing with L-Trp, NA, and NAM does elevate MeNAM concentrations in skeletal muscle tissue, but in discordance with our hypothesis, we found no improvement in mitochondrial respiration capacity in older adults with an impaired physical function.

In the current study, we did not observe an improvement in mitochondrial respiratory capacity in skeletal muscle under $\mathrm{NAD}^{+}$-precursor supplementation compared to the control condition. We have previously shown that the $\mathrm{NAD}^{+}$-precursor acipimox was able to improve mitochondrial function in type 2 diabetes patients (44). However, the lack of effect of $\mathrm{NAD}^{+}$-precursor supplementation in the current study is in accordance with similar recent observations with NR supplementation in obese individuals $(45,46)$ and in older adults $(22)$, and extends this observation to physically compromised older adults. Along the same lines, SPPB performance, energy expenditure, substrate utilization, and exercise efficiency were all unaffected by $\mathrm{NAD}^{+}$-precursor supplementation. These findings are in agreement with Martens et al. (19), who investigated the effect of NR supplementation in healthy, lean, middle-aged to older adults on parameters of physical function such as resting energy expenditure, maximum aerobic capacity, and body composition, and found no differences between NR and placebo. In accordance, Elhassan et al. (22) showed that three weeks of NR supplementation does not increase handgrip strength in older adults. In contrast to our previous finding that NR supplementation resulted in a minor but significant reduction in fat mass in middle-aged obese humans (45), we did not find any effect on body composition upon $\mathrm{NAD}^{+}$-precursor supplementation in the current study. Also, muscle volume was similar in both conditions. Interestingly, although no improvements in physical function were detected upon $\mathrm{NAD}^{+}$-precursor supplementation, perceived physical function was improved upon NAD+. Possibly, 
subtle changes in physical function did occur, which were not detected by our outcome parameters. However, given the small number of volunteers in our study and the fact that this conclusion was based on one out of 9 domains of the RAND-36 survey, one should be cautious in interpreting this result. Future studies should determine both perceived versus actual physical function in (NAD+) supplementation studies.

Blood pressure has also been reported to be susceptible to $\mathrm{NAD}^{+}$-precursor supplementation in humans (19). However, we did not observe a change in SBP, DBP, or MAP under NAD+-precursor supplementation. This is in agreement with our previous NR supplementation study in healthy obese humans, which revealed no improvements in SBP, DBP, or MAP by performing 36-hour blood pressure measurements (45). These data, however, conflict with previous findings from Martens et al. (19) showing improvements in SBP and DBP in healthy middle-aged to older adults following 6 weeks of NR supplementation, which may be explained by discrepancies in the methodologies used to measure blood pressure, such as the higher dose used and duration of the supplementation.

Several studies have demonstrated the ability of $\mathrm{NAD}^{+}$-precursor supplementation to reduce IHL content in mice (47-49). Moreover, Dollerup et al. (21) have reported the tendency for IHL content to decline in obese humans following 12 weeks of NR supplementation. Although this observation did not reach statistical significance, the majority of the NR supplemented group (13 out of 20 participants) had an IHL content greater than 5\% prior to supplementation, and 9 out of these 13 individuals had reduced IHL content after supplementation. Here, we did not observe a beneficial effect of $\mathrm{NAD}^{+}$-precursor supplementation on IHL content. This is possibly due to the already low IHL content in our cohort, with only two participants displaying IHL contents greater than $5 \%$, which is representative of non-alcoholic fatty liver disease (NAFLD) (50), thereby limiting the potential for a reduction in IHL content.

It was previously shown that aging is associated with reduced intracellular $\mathrm{NAD}^{+}$ levels in skeletal muscle in humans $(14,15)$. It is now generally accepted that $\mathrm{NAD}^{+}$ metabolism is a main pathway in the regulation of mitochondrial function in skeletal muscle and would be a target for intervention to stimulate mitochondrial function and thereby prevent age-related decline in physical muscle function. In this light, Pirinen 
et al. have shown that a titration protocol of $250 \mathrm{mg} /$ day to 1000/day $\mathrm{mg}$ of niacin over 4 weeks is able to rescue mitochondrial respiration in adult-onset mitochondrial myopathy patients (51), demonstrating the potential and efficacy of NAD ${ }^{+}$-precursor supplementation in humans with pre-existent conditions. Notably, the window of opportunity for $\mathrm{NAD}^{+}$-precursor supplementation in this population with niacin deficiency by far exceeds the possibilities in populations who are not niacin deficient. Here, we studied a group of community-dwelling, older adults with an impaired physical function and, in measuring the $\mathrm{NAD}^{+}$levels in skeletal muscle tissue, we found that $\mathrm{NAD}^{+}$concentrations in skeletal muscle remained unaffected upon supplementation. We supplemented with three separate NAD+-precursors, being LTrp, NA, and NAM, aiming to take advantage of their respective pathways to contribute to the overall $\mathrm{NAD}^{+}$pool, as illustrated in Figure 2. It has previously been shown that NAM supplementation to cells and mice leads to extended lifespan and healthspan, respectively $(52,53)$. Furthermore, NAM supplementation has been shown to improve glucose metabolism in a rodent model of obesity and type 2 diabetes (54). Recently, de novo biosynthesis of NAD+ from L-Trp has received more attention as a viable dietary source of $\mathrm{NAD}^{+}(25)$. Katsyuba et al. demonstrated that upon the inhibition of the $\alpha$-amino- $\beta$-carboxymuconate- $\varepsilon$-semialdehyde decarboxylase (ACMSD) enzyme in liver and kidney, the conversion of L-Trp to $\mathrm{NAD}^{+}$has the potential to induce SIRT1 activation and, thereby, improve mitochondrial function (25). Here, we did not observe an increase in metabolites belonging to the kynurenine pathway from L-Trp to QA and subsequently to NAAD, a suggested sensitive biomarker of $\mathrm{NAD}^{+}$-precursor supplementation efficacy (55). It is possible that the majority of the L-Trp ingested was consumed by the ACSMD enzyme instead of allowing for spontaneous cyclisation of ACSM into QA and further enzymatic conversion to NAAD and ultimately NAD ${ }^{+}$. However, this remains speculative, as this can only be investigated by acquiring liver tissue, which we have not done in this study. Therefore, more human studies are needed to examine the effects of L-Trp and NAM, given at higher doses, on metabolic health outcomes.

Moreover, we opted to use a low dose of $4 \mathrm{mg}$ of NA daily in order to limit the occurrence of flushing due to its vasoactive properties (56). NAAD is the point of convergence for L-Trp and NA metabolism towards $\mathrm{NAD}^{+}$synthesis. However, NAAD concentrations did not increase in skeletal muscle, suggesting either an 
insufficient dose or the preference of skeletal muscle tissue for another NAD ${ }^{+}$ precursor. Additionally, Liu et al. have shown through isotope-tracer methodology that L-Trp, NA, and NAM are the predominant physiological circulatory NAD+precursors (57). Labeling of L-Trp and NA showed that these precursors are converted to $\mathrm{NAD}^{+}$in the liver and subsequently excreted into the circulation as NAM, and that NAM exchange from the circulation to skeletal muscle tissue is slow, in addition to skeletal muscle tissue exhibiting a low turnover rate of $\mathrm{NAD}^{+}(57)$. This may possibly explain why we did not observe an increase in NAM in skeletal muscle tissue in this study, or any other metabolite of the $\mathrm{NAD}^{+}$-precursors used in our supplementation protocol. Herein, our results contrast with observations of increasing NAAD in skeletal muscle made by us and others previously when supplementing with high doses of NR in obese (45) and aged individuals (22), suggesting that NAAD may be a sensitive, yet specific biomarker for NR supplementation efficacy.

The concentrations of $\mathrm{NAD}^{+}$in skeletal muscle tissue observed in both conditions of the current study appear to be comparable to concentrations found previously by us in a group of healthy, middle-aged, obese individuals supplemented with 1000 $\mathrm{mg} /$ day of NR, and demonstrating unresponsive NAD+ levels to NR supplementation compared to placebo (20). This raises the question whether $\mathrm{NAD}^{+}$levels per se are determinants of efficacy of $\mathrm{NAD}^{+}$-precursor supplementation, or that fluxes of $\mathrm{NAD}^{+}$ are more reliable measures of efficacy. We found that the $\mathrm{NAD}^{+}$concentrations in skeletal muscle tissue were unresponsive to supplementation under differing supplementation strategies in two different populations (the present study and (20)) and observed increased levels of MeNAM in both studies. This observation is also in line with the observations of Elhassan et al. (22) in NR supplemented older adults showing increased MeNAM levels in skeletal muscle, admit unchanged $\mathrm{NAD}^{+}$ concentrations. From the above, one could speculate that the concentration of $\mathrm{NAD}^{+}$ in skeletal muscle tissue is likely to be near the homeostatic maximum in humans in the absence of disease or niacin deficiency, as illustrated by the study of Pirinen et al. (51). This has also been proposed in the 'second hit' theory by Elhassan et al. (22), in which $\mathrm{NAD}^{+}$levels may only decline if chronological age is struck by a second trigger leading to metabolic stress, such as physical inactivity or (chronic) disease. In accordance with this reasoning, we previously showed that supplementation with the synthetic NA-analogue Acipimox increased skeletal muscle 
mitochondrial respiration capacity in individuals with T2DM, who are characterized by a relatively low mitochondrial capacity compared to BMI-matched healthy controls (44). From the above, it could be concluded that there is potential little room for improvement in skeletal muscle $\mathrm{NAD}^{+}$levels in healthy individuals through supplementation with $\mathrm{NAD}^{+}$-precursors, with as a consequence, little or no effect on downstream targets such as mitochondrial function, such as observed in the current study.

We acknowledge that this study is susceptible to limitations. The participants may represent the 'cream of the crop' in terms of older adults with an impaired physical function. As per the 6MWT distances recorded during the screening, the average walking speed in this cohort is $1.09 \mathrm{~m} / \mathrm{s}$, fitting of a healthy older (58) and prefrail population (59). Although individual 6MWT performance met our inclusion criterium, performance on the maximal cycling test yielded a larger variation in overall maximum cycling performance leading to a more heterogeneous cohort than expected. Walking performance is a good predictor of physical function and frailty, co-morbidity, and death (58), but it is hard to distinguish whether walking performance is limited by muscle function or may be limited by other health problems; i.e. a decreased walking performance does not necessarily have to translate to a reduced cycling performance, as joint issues could be the limiting factor and may be less prominent during cycling compared to walking. Secondly, the selected dose of $207.5 \mathrm{mg} /$ day NE (niacin equivalents), although being 12-15 times the RDA for vitamin B-3, may be insufficient to elevate NAD+ levels in humans. The recommended daily allowance (RDA) of niacin for adult humans is set at $16 \mathrm{mg} \mathrm{NE}$ for males and $14 \mathrm{mg}$ for females (17). However, no guidelines exist for older adults specifically. To date, only a small number of studies have looked into the $\mathrm{NAD}^{+}$ boosting ability of lower doses of $\mathrm{NAD}^{+}$-precursors in humans $(23,24)$, and found that doses of $100 \mathrm{mg} /$ day, $250 \mathrm{mg} / \mathrm{day}$, and $300 \mathrm{mg} /$ day NE are able to increase plasma NAD ${ }^{+}$metabolite levels over baseline, such as NAM and MeNAM. However, these studies did not investigate intracellular levels in target tissues such as skeletal muscle. Here, we have supplemented older adults with $207.5 \mathrm{mg}$ NE per day, a level of supplementation emulating increased dietary intake, and show that this is insufficient to increase levels of $\mathrm{NAD}^{+}$and its metabolites in skeletal muscle tissue but does increase skeletal muscle MeNAM levels, suggesting an increase in skeletal 
muscle $\mathrm{NAD}^{+}$metabolism. Further, the duration of the intervention was set at 32 days, which may be relatively short for health benefits to surface. However, we have shown that 30 days of resveratrol supplementation in obese individuals improves mitochondrial respiration capacity (28), supporting the notion that short-term interventions can positively affect human metabolism. Lastly, we cannot exclude that the sample size of the study was too small, although we have previously shown with a similar cross-over design and the methodology used that differences upon nutritional supplementation can be observed with similar sample size. Also, the chance of making a type I error due to testing of multiple study outcomes cannot be excluded.

In summary, we investigated the efficacy of $\mathrm{NAD}^{+}$-precursor supplementation on mitochondrial function aiming to combat the age-dependent decline in skeletal muscle function. We show in community-dwelling, older adults with impaired physical function that of L-Trp, NA, and NAM did increase MeNAM levels in skeletal muscle tissue but did not improve mitochondrial function and skeletal muscle function. Notwithstanding, further research is merited to elucidate the full potential of NAD+precursor supplementation in specific groups with more prominent metabolic dysfunction, such as (morbidly) obese and T2DM individuals. Future studies must also tailor their interventions towards the tissues targeted, optimizing delivery, and taking the fate of the chosen $\mathrm{NAD}^{+}$-precursor into account. 


\section{Chapter 5}

\section{Acknowledgements}

This work was supported by the Top Institute Food and Nutrition (TIFN) research program Mitochondrial Health under grant number $16 \mathrm{NH} 01$.

\section{Duality of interest}

The authors declare that there is no duality of interest associated with this manuscript.

\section{Contribution statement}

N.J.C., L.G., J.d.V., R.H., J.G., B.H., J.H., and P.S. designed research; N.J.C., L.G., C.E.F., and Y.M.H.B. performed research; N.J.C., E.K.M., Y.M.H.B., V.B.S.H., B.H., R.Z.P., R.H.H., J.H., and P.S. analyzed samples and data. N.J.C., J.H., and P.S. wrote the manuscript and all authors critically reviewed the manuscript. P.S. is the guarantor of this work and, as such, had full access to all the data in the study and takes responsibility for the integrity of the data and the accuracy of the data analysis. P.S. is the corresponding author of this work. All authors approved the version to be published. 


\section{REFERENCES}

1. Lopez-Otin C, Blasco MA, Partridge L, Serrano M, Kroemer G. The hallmarks of aging. Cell 2013;153(6):1194-217. doi: 10.1016/j.cell.2013.05.039.

2. Roubenoff R. Sarcopenia and its implications for the elderly. Eur J Clin Nutr 2000;54 Suppl 3:S40-7.

3. Crescenzo R, Bianco F, Mazzoli A, Giacco A, Liverini G, lossa S. Skeletal muscle mitochondrial energetic efficiency and aging. Int J Mol Sci 2015;16(5):10674-85. doi: 10.3390/ijms160510674.

4. Gomes AP, Price NL, Ling AJ, Moslehi JJ, Montgomery MK, Rajman L, White JP, Teodoro JS, Wrann CD, Hubbard BP, et al. Declining NAD(+) induces a pseudohypoxic state disrupting nuclear-mitochondrial communication during aging. Cell 2013;155(7):1624-38. doi: 10.1016/j.cell.2013.11.037.

5. Conley KE, Jubrias SA, Esselman PC. Oxidative capacity and ageing in human muscle. J Physiol 2000;526 Pt 1:203-10. doi: 10.1111/j.1469-7793.2000.t01-100203.x.

6. Kim Y, Triolo M, Hood DA. Impact of Aging and Exercise on Mitochondrial Quality Control in Skeletal Muscle. Oxid Med Cell Longev 2017;2017:3165396. doi: 10.1155/2017/3165396.

7. Andreux PA, Houtkooper RH, Auwerx J. Pharmacological approaches to restore mitochondrial function. Nat Rev Drug Discov 2013;12(6):465-83. doi: 10.1038/nrd4023.

8. Camacho-Pereira J, Tarrago MG, Chini CCS, Nin V, Escande C, Warner GM, Puranik AS, Schoon RA, Reid JM, Galina A, et al. CD38 Dictates Age-Related NAD Decline and Mitochondrial Dysfunction through an SIRT3-Dependent Mechanism. Cell Metab 2016;23(6):1127-39. doi: 10.1016/j.cmet.2016.05.006.

9. Zhu XH, Lu M, Lee BY, Ugurbil $K$, Chen $W$. In vivo NAD assay reveals the intracellular NAD contents and redox state in healthy human brain and their age dependences. Proceedings of the National Academy of Sciences of the United States of America 2015;112(9):2876-81. doi: 10.1073/pnas.1417921112.

10. Yoshino J, Mills KF, Yoon MJ, Imai S. Nicotinamide mononucleotide, a key NAD(+) intermediate, treats the pathophysiology of diet- and age-induced diabetes in mice. Cell Metab 2011;14(4):528-36. doi: 10.1016/j.cmet.2011.08.014.

11. Mills KF, Yoshida S, Stein LR, Grozio A, Kubota S, Sasaki Y, Redpath P, Migaud ME, Apte RS, Uchida K, et al. Long-Term Administration of Nicotinamide Mononucleotide Mitigates Age-Associated Physiological Decline in Mice. Cell Metab 2016;24(6):795-806. doi: 10.1016/j.cmet.2016.09.013.

12. Houtkooper RH, Pirinen E, Auwerx J. Sirtuins as regulators of metabolism and healthspan. Nat Rev Mol Cell Biol 2012;13(4):225-38. doi: 10.1038/nrm3293.

13. Canto C, Auwerx J. Targeting sirtuin 1 to improve metabolism: all you need is NAD(+)? Pharmacol Rev 2012;64(1):166-87. doi: 10.1124/pr.110.003905.

14. de Guia RM, Agerholm M, Nielsen TS, Consitt LA, Sogaard D, Helge JW, Larsen S, Brandauer J, Houmard JA, Treebak JT. Aerobic and resistance exercise training reverses age-dependent decline in $\mathrm{NAD}(+)$ salvage capacity in human skeletal muscle. Physiol Rep 2019;7(12):e14139. doi: 10.14814/phy2.14139.

15. Massudi H, Grant R, Braidy N, Guest J, Farnsworth B, Guillemin GJ. Age-associated changes in oxidative stress and NAD+ metabolism in human tissue. PLoS One 2012;7(7):e42357. doi: 10.1371/journal.pone.0042357. 
16. Mouchiroud L, Houtkooper RH, Auwerx J. NAD(+) metabolism: a therapeutic target for age-related metabolic disease. Crit Rev Biochem Mol Biol 2013;48(4):397-408. doi: 10.3109/10409238.2013.789479.

17. Bogan KL, Brenner C. Nicotinic acid, nicotinamide, and nicotinamide riboside: a molecular evaluation of NAD+ precursor vitamins in human nutrition. Annu Rev Nutr 2008;28:115-30. doi: 10.1146/annurev.nutr.28.061807.155443.

18. Zhang H, Ryu D, Wu Y, Gariani K, Wang X, Luan P, D'Amico D, Ropelle ER, Lutolf $\mathrm{MP}$, Aebersold $\mathrm{R}$, et al. $\mathrm{NAD}(+)$ repletion improves mitochondrial and stem cell function and enhances life span in mice. Science 2016;352(6292):1436-43. doi: 10.1126/science.aaf2693.

19. Martens CR, Denman BA, Mazzo MR, Armstrong ML, Reisdorph N, McQueen MB, Chonchol M, Seals DR. Chronic nicotinamide riboside supplementation is welltolerated and elevates $\mathrm{NAD}(+)$ in healthy middle-aged and older adults. Nat Commun 2018;9(1):1286. doi: 10.1038/s41467-018-03421-7.

20. Remie CME, Roumans KHM, Moonen MPB, Connell NJ, Havekes B, Mevenkamp J, Lindeboom L, de Wit VHW, van de Weijer T, Aarts S, et al. Nicotinamide riboside supplementation alters body composition and skeletal muscle acetylcarnitine concentrations in healthy obese humans. Am J Clin Nutr 2020. doi: 10.1093/ajcn/nqaa072.

21. Dollerup OL, Christensen B, Svart M, Schmidt MS, Sulek K, Ringgaard S, StodkildeJorgensen $\mathrm{H}$, Moller $\mathrm{N}$, Brenner $\mathrm{C}$, Treebak JT, et al. A randomized placebocontrolled clinical trial of nicotinamide riboside in obese men: safety, insulinsensitivity, and lipid-mobilizing effects. Am J Clin Nutr 2018;108(2):343-53. doi: 10.1093/ajcn/nqy132.

22. Elhassan YS, Kluckova K, Fletcher RS, Schmidt MS, Garten A, Doig CL, Cartwright DM, Oakey L, Burley CV, Jenkinson N, et al. Nicotinamide Riboside Augments the Aged Human Skeletal Muscle NAD(+) Metabolome and Induces Transcriptomic and Anti-inflammatory Signatures. Cell Rep 2019;28(7):1717-28 e6. doi: 10.1016/j.celrep.2019.07.043.

23. Irie J, Inagaki E, Fujita M, Nakaya H, Mitsuishi M, Yamaguchi S, Yamashita K, Shigaki S, Ono T, Yukioka H, et al. Effect of oral administration of nicotinamide mononucleotide on clinical parameters and nicotinamide metabolite levels in healthy Japanese men. Endocr J 2020;67(2):153-60. doi: 10.1507/endocrj.EJ19-0313.

24. Conze D, Brenner C, Kruger CL. Safety and Metabolism of Long-term Administration of NIAGEN (Nicotinamide Riboside Chloride) in a Randomized, Double-Blind, Placebo-controlled Clinical Trial of Healthy Overweight Adults. Sci Rep 2019;9(1):9772. doi: 10.1038/s41598-019-46120-z.

25. Katsyuba E, Mottis A, Zietak M, De Franco F, van der Velpen V, Gariani K, Ryu D, Cialabrini L, Matilainen $\mathrm{O}$, Liscio $\mathrm{P}$, et al. De novo $\mathrm{NAD}(+)$ synthesis enhances mitochondrial function and improves health. Nature 2018;563(7731):354-9. doi: 10.1038/s41586-018-0645-6.

26. Grootswagers P, Mensink M, Berendsen AAM, Deen CPJ, Kema IP, Bakker SJL, Santoro A, Franceschi C, Meunier N, Malpuech-Brugere C, et al. Vitamin B-6 intake is related to physical performance in European older adults: results of the New Dietary Strategies Addressing the Specific Needs of the Elderly Population for Healthy Aging in Europe (NU-AGE) study. Am J Clin Nutr 2021. doi: 10.1093/ajcn/nqaa368. 
27. Baecke JA, Burema J, Frijters JE. A short questionnaire for the measurement of habitual physical activity in epidemiological studies. Am J Clin Nutr 1982;36(5):93642. doi: 10.1093/ajcn/36.5.936.

28. de Ligt $M$, Bruls $Y M H$, Hansen J, Habets MF, Havekes B, Nascimento EBM, Moonen-Kornips E, Schaart G, Schrauwen-Hinderling VB, van Marken Lichtenbelt W, et al. Resveratrol improves ex vivo mitochondrial function but does not affect insulin sensitivity or brown adipose tissue in first degree relatives of patients with type 2 diabetes. Mol Metab 2018;12:39-47. doi: 10.1016/j.molmet.2018.04.004.

29. Edtion ed. Dietary Reference Intakes for Thiamin, Riboflavin, Niacin, Vitamin B6, Folate, Vitamin B12, Pantothenic Acid, Biotin, and Choline. Washington (DC), 1998.

30. Fukuwatari T, Shibata K. Nutritional aspect of tryptophan metabolism. Int J Tryptophan Res 2013;6(Suppl 1):3-8. doi: 10.4137/IJTR.S11588.

31. Kuipers H, Verstappen FT, Keizer HA, Geurten P, van Kranenburg G. Variability of aerobic performance in the laboratory and its physiologic correlates. Int J Sports Med 1985;6(4):197-201. doi: 10.1055/s-2008-1025839.

32. VanderZee KI, Sanderman R, Heyink JW, de Haes H. Psychometric qualities of the RAND 36-Item Health Survey 1.0: a multidimensional measure of general health status. Int J Behav Med 1996;3(2):104-22. doi: 10.1207/s15327558ijbm0302_2.

33. VanderZee KI, Sanderman R. Het meten van de algemene gezondheidstoestand met de RAND-36, een handleiding. UMGC / Rijksuniversiteit Groningen, Research Institute SHARE 2012; Tweede herziene druk.

34. Lindeboom L, Nabuurs Cl, Hesselink MK, Wildberger JE, Schrauwen P, SchrauwenHinderling VB. Proton magnetic resonance spectroscopy reveals increased hepatic lipid content after a single high-fat meal with no additional modulation by added protein. Am J Clin Nutr 2015;101(1):65-71. doi: 10.3945/ajcn.114.094730.

35. Guralnik JM, Simonsick EM, Ferrucci L, Glynn RJ, Berkman LF, Blazer DG, Scherr PA, Wallace RB. A short physical performance battery assessing lower extremity function: association with self-reported disability and prediction of mortality and nursing home admission. Journal of gerontology 1994;49(2):M85-94.

36. Bergstrom J. Percutaneous needle biopsy of skeletal muscle in physiological and clinical research. Scand J Clin Lab Invest 1975;35(7):609-16.

37. Hoeks J, van Herpen NA, Mensink M, Moonen-Kornips E, van Beurden D, Hesselink MK, Schrauwen P. Prolonged fasting identifies skeletal muscle mitochondrial dysfunction as consequence rather than cause of human insulin resistance. Diabetes 2010;59(9):2117-25. doi: 10.2337/db10-0519.

38. Phielix E, Meex R, Ouwens DM, Sparks L, Hoeks J, Schaart G, Moonen-Kornips E, Hesselink MK, Schrauwen P. High oxidative capacity due to chronic exercise training attenuates lipid-induced insulin resistance. Diabetes 2012;61(10):2472-8. doi: $10.2337 / \mathrm{db} 11-1832$.

39. Weir JB. New methods for calculating metabolic rate with special reference to protein metabolism. J Physiol 1949;109(1-2):1-9.

40. Matomaki P, Linnamo V, Kyrolainen H. A Comparison of Methodological Approaches to Measuring Cycling Mechanical Efficiency. Sports Med Open 2019;5(1):23. doi: 10.1186/s40798-019-0196-x.

41. van Vlies N, Tian L, Overmars H, Bootsma AH, Kulik W, Wanders RJ, Wood PA, Vaz FM. Characterization of carnitine and fatty acid metabolism in the long-chain acylCoA dehydrogenase-deficient mouse. Biochem J 2005;387(Pt 1):185-93. doi: 10.1042/BJ20041489. 
42. Kato T, Berger SJ, Carter JA, Lowry OH. An enzymatic cycling method for nicotinamide-adenine dinucleotide with malic and alcohol dehydrogenases. Anal Biochem 1973;53(1):86-97. doi: 10.1016/0003-2697(73)90409-0.

43. Timmers S, Konings E, Bilet L, Houtkooper RH, van de Weijer T, Goossens GH, Hoeks J, van der Krieken S, Ryu D, Kersten S, et al. Calorie restriction-like effects of 30 days of resveratrol supplementation on energy metabolism and metabolic profile in obese humans. Cell Metab 2011;14:612-22.

44. van de Weijer T, Phielix E, Bilet L, Williams EG, Ropelle ER, Bierwagen A, Livingstone R, Nowotny P, Sparks LM, Paglialunga S, et al. Evidence for a direct effect of the NAD+ precursor acipimox on muscle mitochondrial function in humans. Diabetes 2015;64(4):1193-201. doi: 10.2337/db14-0667.

45. Remie CM, Roumans KH, Moonen MP, Connell NJ, Havekes B, Mevenkamp J, Lindeboom L, de Wit VH, van de Weijer T, Aarts SA. Nicotinamide riboside supplementation alters body composition and skeletal muscle acetylcarnitine concentrations in healthy obese humans. The American Journal of Clinical Nutrition 2020.

46. Dollerup OL, Chubanava S, Agerholm M, Sondergard SD, Altintas A, Moller AB, Hoyer KF, Ringgaard S, Stodkilde-Jorgensen H, Lavery GG, et al. Nicotinamide riboside does not alter mitochondrial respiration, content or morphology in skeletal muscle from obese and insulin-resistant men. J Physiol 2020;598(4):731-54. doi: 10.1113/JP278752.

47. Zhou CC, Yang X, Hua X, Liu J, Fan MB, Li GQ, Song J, Xu TY, Li ZY, Guan YF, et al. Hepatic $N A D(+)$ deficiency as a therapeutic target for non-alcoholic fatty liver disease in ageing. Br J Pharmacol 2016;173(15):2352-68. doi: 10.1111/bph.13513.

48. Gariani K, Menzies KJ, Ryu D, Wegner CJ, Wang X, Ropelle ER, Moullan N, Zhang $H$, Perino $A$, Lemos $V$, et al. Eliciting the mitochondrial unfolded protein response by nicotinamide adenine dinucleotide repletion reverses fatty liver disease in mice. Hepatology 2016;63(4):1190-204. doi: 10.1002/hep.28245.

49. Trammell SA, Weidemann BJ, Chadda A, Yorek MS, Holmes A, Coppey LJ, Obrosov A, Kardon RH, Yorek MA, Brenner C. Nicotinamide Riboside Opposes Type 2 Diabetes and Neuropathy in Mice. Sci Rep 2016;6:26933. doi: 10.1038/srep26933.

50. Gaggini M, Morelli M, Buzzigoli E, DeFronzo RA, Bugianesi E, Gastaldelli A. Nonalcoholic fatty liver disease (NAFLD) and its connection with insulin resistance, dyslipidemia, atherosclerosis and coronary heart disease. Nutrients 2013;5(5):154460. doi: 10.3390/nu5051544.

51. Pirinen E, Auranen M, Khan NA, Brilhante V, Urho N, Pessia A, Hakkarainen A, Kuula J, Heinonen U, Schmidt MS, et al. Niacin Cures Systemic NAD(+) Deficiency and Improves Muscle Performance in Adult-Onset Mitochondrial Myopathy. Cell Metab 2020;31(6):1078-90 e5. doi: 10.1016/j.cmet.2020.04.008.

52. Lim CS, Potts M, Helm RF. Nicotinamide extends the replicative life span of primary human cells. Mech Ageing Dev 2006;127(6):511-4. doi: 10.1016/j.mad.2006.02.001.

53. Mitchell SJ, Bernier M, Aon MA, Cortassa S, Kim EY, Fang EF, Palacios HH, Ali A, Navas-Enamorado I, Di Francesco A, et al. Nicotinamide Improves Aspects of Healthspan, but Not Lifespan, in Mice. Cell Metab 2018;27(3):667-76 e4. doi: 10.1016/j.cmet.2018.02.001.

54. Yang SJ, Choi JM, Kim L, Park SE, Rhee EJ, Lee WY, Oh KW, Park SW, Park CY. Nicotinamide improves glucose metabolism and affects the hepatic NAD-sirtuin 
pathway in a rodent model of obesity and type 2 diabetes. J Nutr Biochem 2014;25(1):66-72. doi: 10.1016/j.jnutbio.2013.09.004.

55. Trammell SA, Schmidt MS, Weidemann BJ, Redpath P, Jaksch F, Dellinger RW, Li $Z$, Abel ED, Migaud ME, Brenner C. Nicotinamide riboside is uniquely and orally bioavailable in mice and humans. Nat Commun 2016;7:12948. doi: 10.1038/ncomms12948.

56. Benyo Z, Gille A, Bennett CL, Clausen BE, Offermanns S. Nicotinic acid-induced flushing is mediated by activation of epidermal langerhans cells. Mol Pharmacol 2006;70(6):1844-9. doi: 10.1124/mol.106.030833.

57. Liu L, Su X, Quinn WJ, 3rd, Hui S, Krukenberg K, Frederick DW, Redpath P, Zhan L, Chellappa K, White E, et al. Quantitative Analysis of NAD Synthesis-Breakdown Fluxes. Cell Metab 2018;27(5):1067-80 e5. doi: 10.1016/j.cmet.2018.03.018.

58. Abellan van Kan G, Rolland Y, Andrieu S, Bauer J, Beauchet O, Bonnefoy M, Cesari M, Donini LM, Gillette Guyonnet S, Inzitari M, et al. Gait speed at usual pace as a predictor of adverse outcomes in community-dwelling older people an International Academy on Nutrition and Aging (IANA) Task Force. J Nutr Health Aging 2009;13(10):881-9. doi: 10.1007/s12603-009-0246-z.

59. Tompkins BA, DiFede DL, Khan A, Landin AM, Schulman IH, Pujol MV, Heldman AW, Miki R, Goldschmidt-Clermont PJ, Goldstein BJ, et al. Allogeneic Mesenchymal Stem Cells Ameliorate Aging Frailty: A Phase II Randomized, Double-Blind, PlaceboControlled Clinical Trial. J Gerontol A Biol Sci Med Sci 2017;72(11):1513-22. doi: 10.1093/gerona/glx137. 


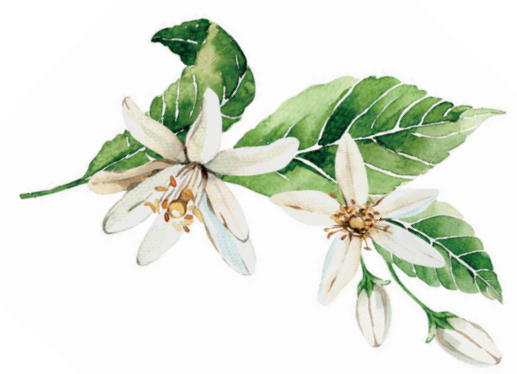

\section{CHAPTER 6}

\section{Type II muscle fibre properties are not associated with balance recovery following large perturbations during walking in young and older adults}

Lotte Grevendonk*, Christopher McCrum*, Gert Schaart, Esther Moonen-Kornips, Johanna A. Jörgensen, Anne Gemmink, Kenneth Meijer, Joris Hoeks

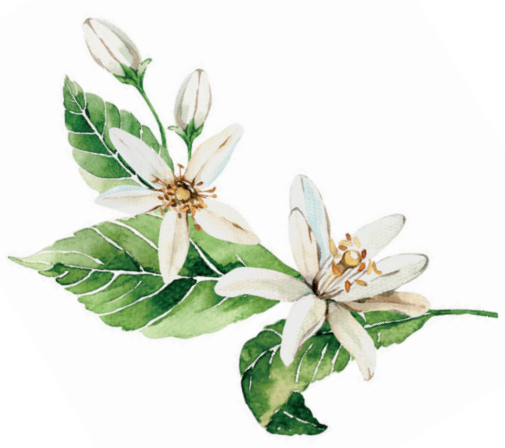




\section{Chapter 6}

\section{ABSTRACT}

Falls among older adults are often attributed to declining muscle strength with ageing. Associations between muscle strength and balance control have been reported, but the evidence for, and key mechanisms of resistance exercise in fall prevention are unclear. No studies have directly examined the relationship between muscle fibre characteristics and reactive balance control. Here, we address whether or not Type II muscle fibre characteristics associate with reactive balance during walking in young and older adults with varying muscle fibre type composition. We analyse muscle biopsy-derived fibre characteristics and stability during a treadmillbased walking perturbation (trip-like) task of healthy young adults, healthy, normally active older adults, trained older adults and physically impaired older adults. We find no significant associations between Type II muscle fibre properties and reactive balance during walking, indicating that practitioners and researchers should consider more than just the muscle tissue properties when assessing and intervening on fall risk. 


\section{INTRODUCTION}

Loss of muscle mass in humans after the age of 50 years is largely attributable to declines in lower body muscle mass [1], which has implications for mobility in older age. Specifically, the leg extensor muscle-tendon groups critical for gait [2-4] show reduced muscle strength and altered tendon mechanical properties in older, compared to younger adults [5-8]. Lower limb muscle strength [9-12], power $[11,13,14]$ and quality $[15,16]$ have been associated with daily life falls incidence in older adults, although findings are not unequivocal $[12,13,17]$. The majority of falls in community-dwelling older adults occur following large balance disturbances (like trips and slips) during walking [18-25]. Small to moderate associations have been reported between lower limb muscle strength and balance recovery performance following laboratory-based lean-and-release [26-30], trip [31-34] and slip [35,36] perturbations simulating common causes of falls. Regarding interventions, there is weak or mixed evidence that general strength training alone can improve balance [37-39] and reduce falls [40], and whether a direct cause-effect relationship between muscle strength and balance performance exists has been questioned [41].

The loss in muscle strength and function with increasing age may occur due to muscle fibre atrophy and muscle fibre loss [42-45]. Age-related decline in muscle mass seems to be primarily attributable to an atrophy of specifically Type II muscle fibres [46-51]. Older age is also associated with a change in the expression of myosin heavy chain isoforms (MHC) in favor of slow MHC I, reflecting the shift towards a slower fibre type profile (Type I) and the selective atrophy of Type II fibres [51-53]. One study on older female participants with and without hip fractures reported that age and hip fracture history had significant individual and accumulating effects on Type II muscle fibre size (decreasing with age and fall-related hip fracture) [46]. Encouragingly, physical activity may prevent or slow this age-related atrophy of Type II fibres [49] and it has been reported that increases in muscle mass as a result of resistance exercise in old age is attributable to hypertrophy of Type II fibres specifically (and almost exclusively)[48]. In contrast, in endurance-trained older adults, greater proportions of Type I fibres have been observed when compared to strength-trained older adults, untrained older adults and untrained young individuals [54-56]. 
Together, these data suggest that muscle fibre characteristics are an important factor in balance performance and falls. Despite this, there are no studies, to our knowledge, directly examining the relationship between muscle fibre characteristics and reactive balance control in the literature. In this study, we address for the first time whether or not Type II muscle fibre characteristics associate with reactive balance during walking in young and older adults with varying muscle fibre type composition. To do this, we analyse muscle biopsy-derived fibre characteristics and stability during a treadmill-based gait perturbation task of healthy young adults, healthy, normally active older adults, trained older adults and physically impaired older adults. Based on previous studies, we hypothesised that there would be a small to moderate relationship between Type II muscle fibre characteristics (size and proportion) and stability recovery following large perturbations during walking (number of recovery steps needed following a novel perturbation). We additionally explored these relationships after repeated perturbations to examine the potential role of Type II muscle fibre characteristics in adaptability of stability recovery responses.

\section{METHODS}

\section{Participants}

The data and analyses in the current secondary analysis were part of a larger study ( $n=59$; chapter 3 ), which was approved by the institutional Medical Ethical Committee, conducted in accordance with the declaration of Helsinki and registered at clinicaltrials.gov (NCT03666013). All participants provided their written informed consent. Some data have been reported in our previous publication addressing different research questions (chapter 3). For the current secondary analysis, the data of all participants who underwent muscle biopsies and completed the walking perturbation experiment in the larger study were included. This resulted in a sample of 52 participants including 14 young ( 7 male and 7 female) and 38 older (22 male and 16 female) individuals. Due to this being a secondary analysis, no formal power calculation was conducted to determine the sample size. However, sample sizes of 46 and 47 will achieve approximately $80 \%$ power with a significance level of 0.05 to detect Pearson and Spearman Correlation Coefficients of 0.4 , which we consider reasonable for the purpose of the current analysis. 
Prior to inclusion, all participants underwent a medical screening that included a medical questionnaire, a physical examination by a physician, and an assessment of physical function by means of a Short Physical Performance Battery [57]. After screening, participants were assigned to the following groups: Young individuals with normal physical activity (Y, 20 - 30 years), older adults with normal physical activity (O, 65 - 80 years), trained older adults (TO, 65 - 80 years) and older adults with impaired physical function (IO, 65 - 80 years). Participants were considered normally physically active if they completed no more than one structured exercise session per week, whereas participants were considered trained if they engaged in at least 3 structured exercise sessions of at least 1 hour each per week for an uninterrupted period of at least one year. Participants were classified as older adults with impaired physical function $(\mathrm{IO})$ in case of an SPPB score of $\leq 9$.

\section{Body Composition and Muscle Volume}

Body composition (fat and fat-free mass) was determined at 8 AM after an overnight fast from $10 \mathrm{PM}$ the previous evening using air displacement plethysmography (BodPod®, COSMED, Inc., Rome, Italy) [58]. To measure muscle volume, a 3T whole body MRI scanner was used (Achieva 3T-X; Philips Healthcare, Best, The Netherlands). Participants were positioned in the supine position in the MR scanner (feet first) to determine muscle volume of the upper leg using the body coil. Subsequently, a series of T1-weighted images were acquired of the upper leg (slice thickness of $10 \mathrm{~mm}$, no gap between slices, in-plane resolution of $0.78 \times 0.78 \mathrm{~mm})$. A custom-written MATLAB script was used to automatically segment adipose and muscle tissue. Thereby each image was normalised, and a histogram calculated. Histogram peaks corresponding to muscle tissue grey values were summed up, resulting in the count of muscle pixels. Muscle volume was calculated by voxel size times the number of muscle pixels: Vol_muscle $=$ Vox_size* $N$ muscle pixels. Every slice was reviewed individually and in case of a clear notable deviation, a manual threshold correction for muscle grey values was performed. The muscle segmentation was performed in the consecutive slices between the starting point (tendon attachment) of the $m$. rectus femoris and of the $m$. gluteus maximus. 


\section{Habitual physical activity}

Habitual physical activity was estimated using an ActivPAL activity monitor (PAL Technologies, Glasgow, Scotland) for five consecutive days, including two weekend days. The total amount of steps per day was measured, as well as the total stepping time in proportion to waking time, determined according to van der Berg et al. [59]. Stepping time (i. e., physical activity) was then further classified into high-intensity physical activity (HPA; minutes with a step frequency $>110$ steps/min in proportion to waking time) [60].

\section{Muscle Strength}

Maximum voluntary knee extension and flexion torque was measured using the Biodex System 3 Pro dynamometer (Biodex® Medical Systems, Inc., Shirley, NY, USA). Participants were stabilized with shoulder, leg, and abdominal straps to prevent compensatory movement and the test was performed with the left leg in all participants. Participants performed three $5 \mathrm{~s}$ maximal extensions and flexions with a 30 s rest period in between trials. The knee position was fixed at a $70^{\circ}$ angle. Maximal voluntary isometric knee-extensor and knee-flexor torque was defined as the average of the highest two out of three peak torques.

Participants also performed 30 consecutive extension and flexion movements (range of motion 120 degrees/s). The peak torque of each extension and flexion was recorded and the maxima were defined as the highest peak. For both protocols, maximal torque was recorded in Newton-meters and corrected for bodyweight $(\mathrm{Nm} / \mathrm{kg})$.

\section{Muscle Biopsy and Fibre Typing}

A biopsy specimen was taken from the vastus lateralis muscle under local anesthesia (1.0\% lidocaine without epinephrine), according to the Bergström method [61]. Muscle tissue was immediately frozen in melting isopentane cooled with liquid nitrogen for immunohistochemistry and stored at $-80^{\circ} \mathrm{C}$ degrees until analysis. Fresh $5 \mu \mathrm{m}$-thick cryosections were treated for five minutes with $0.25 \%$ Triton $\mathrm{X}-100$ in phosphate-buffered saline and incubated for 45 minutes at room temperature with a 
mouse monoclonal MHC1-antibody (A.4840; Developmental Studies Hybridoma Bank, lowa City, IO) and with the basal membrane marker laminin (L9393; Sigma, Zwijndrecht, The Netherlands). Thereafter, sections were incubated for 45 minutes with the appropriate secondary antibodies goat anti-mouse lgM AlexaFluor488 (A21042; Invitrogen Thermo Fisher, Breda, The Netherlands) and goat anti-rabbit IgG AlexaFluor555 (A21428; Invitrogen Thermo Fisher) and mounted with Mowiol. All sections were captured using a Nikon E800 fluorescence microscope (Nikon Instruments, Amsterdam, The Netherlands [62]) and images were analyzed in ImageJ [63]. Cell membranes were background corrected and threshold was set manually to create a binary image. Subsequently, the original cell membrane image (laminin staining) was added to the binary image of the cell membranes to check whether all cell membranes in the binary image were closed. If not, cell membranes were closed manually. Fibre size was determined by measuring the cross-sectional area (CSA) and fibre type distribution was expressed as a percentage with respect to the number of fibres and as a percentage of CSA (\% fibre type I/II area relative to total CSA).

\section{Stability during Perturbed Walking}

The walking measurements were conducted using the Computer Assisted Rehabilitation Environment Extended (CAREN; Motekforce Link, Amsterdam). The system includes a dual-belt force plate-instrumented treadmill $(1000 \mathrm{~Hz})$, a 12 camera Vicon Nexus motion capture system $(100 \mathrm{~Hz}$; Vicon Motion Systems, Oxford, UK) and a $180^{\circ}$ virtual environment providing optic flow. A safety harness connected to an overhead frame was worn by participants at all times. Six retroflective markers were attached to anatomical landmarks (C7, sacrum, left and right trochanter and left and right hallux).

Participants were already accustomed to the CAREN setup, as they had previously completed an instrumented six-minute walk test as part of the larger study. To further ensure participants were comfortable and familiarised to the measurement setup and conditions, the perturbation measurement session began with unrecorded fixedspeed walking trials of two minutes at speeds of $0.4 \mathrm{~m} / \mathrm{s}$ up to $1.8 \mathrm{~m} / \mathrm{s}$ in $0.2 \mathrm{~m} / \mathrm{s}$ increments. Following a break, the same fixed-speed walking trials were repeated 
and recorded. During another break, the data from these trials were used to calculate a stability-normalised walking speed for each participant individually for use in the subsequent walking perturbation trial, which was set for a margin of stability (MoS; see below; [64]) of 0.05m as described previously [65], ensuring a comparable baseline walking stability for all groups [66,67]. Next, participants completed the perturbation trial which started with three to four minutes of unperturbed walking at the stability-normalised walking speed, followed by 10 unannounced unilateral treadmill belt acceleration perturbations (every 30 to 90 seconds). The first and tenth accelerations were applied to the right leg, while the second to ninth accelerations were applied to the left leg as described previously $[66,67]$. Participants were told that they would complete a walking balance challenge and to try to continue walking as normally as possible. The participants were unaware of the specifics of the perturbations and no warnings or cues were given during the trial. For the current study, the first perturbations to each limb were analysed (Pert1R and Pert2L), representing novel disturbances, as well as the ninth perturbation (final left leg perturbation; Pert9L) to indicate adaptation in gait following eight repeated perturbations.

Data processing was conducted in MATLAB (2016a, The MathWorks, Inc., Natick). The three-dimensional coordinates of the markers were filtered using a low pass second order Butterworth filter (zero-phase) with a $12 \mathrm{~Hz}$ cut-off frequency. Foot touchdown and toe-off were detected using a combined method of force plate data (50 $\mathrm{N}$ threshold) and foot marker data [68], as described in detail previously [69]. The anteroposterior MoS at foot touchdown were calculated as the anteroposterior distance between the anterior boundary of the base of support (BoS) and the extrapolated centre of mass (X $\mathrm{CoM}$ ) [64], adapted for our validated reduced kinematic model [70] and treadmill walking [71], in the same manner as our previous studies [65-67]. The MoS was calculated for the following steps: the mean MoS of the eleventh to second last step before each perturbation (Base); the final step before each perturbation (Pre); and the first eight recovery steps following each perturbation (Post1-8). In order to determine the number of steps to recover to baseline stability (up to the eighth recovery step), two methods were used. The first, as used in our previous study [67], used a $0.05 \mathrm{~m}$ threshold - if the recovery step MoS value was within $0.05 \mathrm{~m}$ of the Base MoS, this was heuristically considered not meaningfully 
different to unperturbed baseline walking. The second method, rather than using a standard value for all participants, used an individual threshold of three standard deviations of the 10 pre-perturbation steps used to calculate Base. This accounted for those individuals with either much lower or higher variability in their unperturbed walking for whom the $0.05 \mathrm{~m}$ threshold might have been inappropriate. As there is no accepted standard for establishing the number of recovery steps, we have included and analysed both outcomes.

\section{Statistics}

Normality for all data was checked with Shapiro-Wilk tests, which determined whether parametric or non-parametric analyses would be used. Group differences in the parameters were assessed using either one-way ANOVAs with Tukey's multiple comparisons tests or Kruskal-Wallis tests with Dunn's multiple comparisons tests. All analyses involving the number of recovery steps included analyses using the standard $0.05 \mathrm{~m}$ threshold (Standard Threshold) and the individualised, three standard deviation threshold (Individual Threshold). Spearman correlations were conducted to assess the relationships between the recovery steps required by participants during Pert1R, Pert2 $\mathrm{L}$ and Pert9 $\mathrm{L}$ and the muscle fibre properties (percentage of Type II fibres, mean Type II fibre CSA and the percentage of CSA taken up by Type II fibres). Spearman correlations were also conducted for the recovery steps required and the muscle volume and strength measures. Correlations with Pert1R and Pert2L were used to determine associations with balance recovery following a novel perturbation (similar unexpected and unfamiliar situation to how falls occur in daily life) whereas associations with Pert9L were used to determine associations with adaptability of the stability recovery responses. $\alpha$ was set at 0.05 . Correlation coefficients were interpreted as follows: 0-0.1: Negligible; 0.1-0.3: Small; 0.3-0.5: Moderate; 0.5 and above: Large [72], although these may be overestimates in comparison to the field of gerontology, where there is evidence that Pearson's $r=$ $0.12,0.20$, and .032 may better represent small medium and large effects [73]. Statistical analyses were conducted, and figures were prepared using GraphPad Prism version 9 for Windows (GraphPad Software, LLC, San Diego, California, USA). 


\section{Chapter 6}

\section{RESULTS}

Participant Characteristics

Of the 52 participants who were included in this study, one participant was excluded due to problems in both walking motion capture (noise and marker gaps) and muscle biopsy (not enough tissue), and a further three participants were excluded due to issues with the muscle biopsy (not enough useable tissue was collected). The remaining 48 participants' data were included in the analyses and their characteristics, arranged per group, are displayed in Table 1. Note that we did not specifically aim to have groups of a specific size, as the groups themselves were not related to our primary aims. Rather we wanted to achieve a sample that included a wide range of muscle fibre type characteristics and stability recovery performance that could provide insight into the associations between these parameters. Due to technical issues, walking data from Pert1 $\mathrm{R}$ for one participant of group $\mathrm{O}$, walking data from Pert2L for one participant of group TO and MRI-derived muscle volume data for one participant of group TO were excluded.

Groups $O$ and $Y$ possessed similar physical activity levels ( $10 \mathrm{~K}$ steps/day and similar active time in high-intensity activities; Table 1). Group TO, in comparison were more active ( $13 \mathrm{~K}$ steps/day and $4.9 \%$ active time was at high intensity; Table 1) and were mainly involved in endurance sports and training, whereas Group IO were less active $(\sim 7.5 \mathrm{~K}$ steps/day and $1.6 \%$ active time at high intensity; Table 1$)$. 
Table 1: Participant characteristics, presented as mean (SD).

\begin{tabular}{|c|c|c|c|c|}
\hline & $\begin{array}{l}\text { Young, normal } \\
\text { physical } \\
\text { activity }(Y)\end{array}$ & $\begin{array}{l}\text { Older adults, } \\
\text { normal } \\
\text { physical } \\
\text { activity }(0)\end{array}$ & $\begin{array}{l}\text { Physically } \\
\text { impaired older } \\
\text { adults (IO) }\end{array}$ & $\begin{array}{l}\text { Trained older } \\
\text { adults (TO) }\end{array}$ \\
\hline $\mathbf{N}$ & 12 & 16 & 3 & 17 \\
\hline Male & 7 & 8 & 2 & 11 \\
\hline Age [years] & $23.9(2.2)$ & $70.6(3)$ & $69.7(4.2)$ & $67.8(2.4)$ \\
\hline BMI $\left[\mathrm{kg} / \mathrm{m}^{2}\right]$ & $22.4(3.3)$ & $25.8(3.4)$ & $28.4(1.8)$ & $23.8(1.8)$ \\
\hline Height [cm] & $176(7.4)$ & 169 (11.3) & $171(4.4)$ & $171.2(7.2)$ \\
\hline Weight [kg] & $69.3(11.5)$ & $74.0(12.3)$ & $82.9(2.4)$ & $70(7.5)$ \\
\hline Fat mass [\%] & $22.6(8.4)$ & $33.3(9.5)$ & $37(10.7)$ & $25.9(8.1)$ \\
\hline SPPB $4 \mathrm{~m}$ walk & $1.1(0.2)$ & $1.2(0.2)$ & $1.0(0.3)$ & $1.3(0.2)$ \\
\hline SPPB chair test & $9.2(2.7)$ & $10.2(1.4)$ & $13.7(7.1)$ & $8.9(2.1)$ \\
\hline Steps/day & $10487(3134)$ & $10007(2885)^{\ddagger}$ & 7594 (1851) & 13604 (6218) \\
\hline $\begin{array}{l}\text { HPA time/wake } \\
\text { time (\%) }\end{array}$ & $2.6(1.7)^{*}$ & $2.1(1.2)^{\ddagger}$ & $1.6(0.1)$ & $4.9(3.9)$ \\
\hline
\end{tabular}

Type I and II Muscle Fibre Characteristics

In order to evaluate the muscle fibre type characteristics of the muscle biopsy samples, a mean (SD) of 454(260) muscle fibres were analysed per participant. Representative fluorescence microscope images used for fibre type analyses are displayed in Fig. 1. In order to explore the range in the characteristic, one-way ANOVAs were applied and did not reveal a significant group effect on Type I or II muscle fibre percentage $(F(3,44)=2.8, P=0.051)$, Type I fibre CSA $(F(3,44)=0.8$, $\mathrm{P}=0.5)$ or Type II fibre $\operatorname{CSA}(\mathrm{F}(3,44)=1.9, \mathrm{P}=0.14)$, although group TO tended to have lower Type II fibre percentage (Fig. 1). A one-way ANOVA did reveal a significant effect of group on the percentage of total CSA taken up by Type I and II fibres $(F(3,44)=4.5, P=0.007)$, with Tukey's multiple comparisons tests revealing significantly lower values for group TO vs. $Y$ (adjusted $P=0.008$ ) and $O$ (adjusted $P=0.036)$, as indicated in Fig. 1. While group comparisons were not always significant, these data confirm a range of muscle fibre type characteristics were 


\section{Chapter 6}

present in our analysed participants, that there were no significant differences between groups $\mathrm{Y}$ and $\mathrm{O}$ and that group TO have an altered fibre type composition favouring Type I fibres. As can be seen in Fig. 1, Type II fibre mean CSA appeared to be lower in all older adult groups compared to group $\mathrm{Y}$, despite a non-significant one-way ANOVA. Post hoc Mann Whitney tests comparing group $\mathrm{Y}(\mathrm{n}=12$, Median $\left.=5477 \mu^{2}\right)$ with all older participants collapsed into one group $(n=36$, Median $\left.=3509 \mu \mathrm{m}^{2}\right)$ and with Group $\mathrm{O}\left(\mathrm{n}=16\right.$, Median $\left.=3500 \mu \mathrm{m}^{2}\right)$ revealed significant differences in Type II fibre mean CSA $(U=114, P=0.014$ and $U=49, P=0.029$, respectively). 

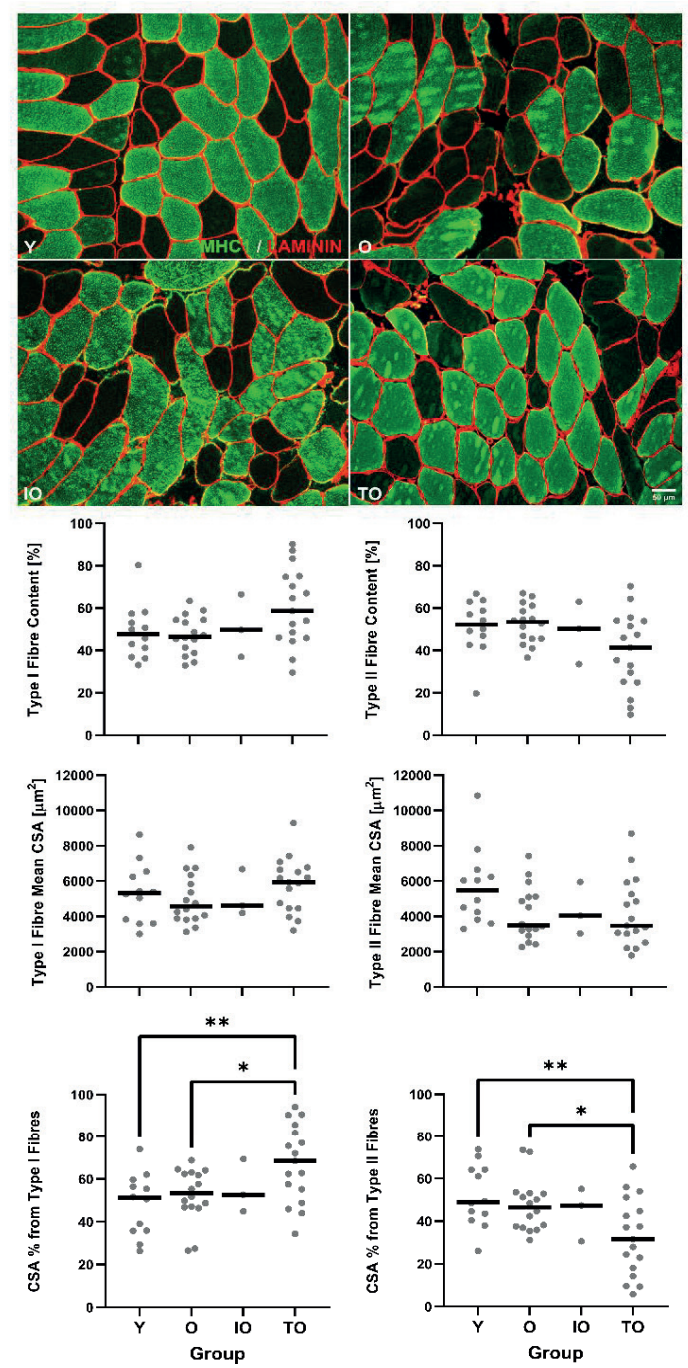

Figure 1: Top Panel: Representative fluorescence microscope images used for fibre type analyses from young adults $(Y)$, older adults with normal physical activity $(\mathrm{O})$, trained older adults (TO) and physically impaired older adults (IO). Basal membrane (laminin; red); type 1 fibres (MHC1; green) and type 2 fibres (no stain / black). Each participant shown has the median value of average fibre CSA within their group. Bottom Panel: Group median and individual Type I and II muscle fibre characteristics. *: $P=0.036^{* *}: P=0.008$ 
Muscle Volume, Strength Characteristics and Perturbation Recovery Steps

A one-way ANOVA did not reveal a significant effect of group on upper leg muscle volume $(F(3,43)=0.74, P=0.35)$. A significant group effect on peak isometric knee extension torque was found $(F(3,44)=3.06, P=0.038)$ with significantly lower values for group $O$ vs. $Y$ (adjusted $P=0.0287$ ). Kruskal-Wallis tests did not reveal a significant group effect on peak isometric knee flexion torque $(H(3)=4.68, P=0.197)$ but did reveal significant group effects on peak isokinetic knee extension $(H(3)=25.96, P<0.0001)$ and flexion $(H(3)=25.98, P<0.0001)$ torque, with significantly greater peak isokinetic knee extension torque in group $\mathrm{Y}$ vs. $\mathrm{O}$ (adjusted $\mathrm{P}<0.0001$ ), vs. $I O$ (adjusted $P=0.0024$ ) and vs. TO (adjusted $P=0.0178$ ) and significantly greater peak isokinetic knee flexion torque in group $\mathrm{Y}$ vs. $\mathrm{O}$ (adjusted $\mathrm{P}<0.0001$ ) and vs. 10 (adjusted $\mathrm{P}=0.0022$ ). Similar to the muscle fibre type characteristics, these data confirm a range of muscle strength values in our analysed participants. The muscle volume and strength data are displayed in the supplemental figures 1 and 2 .

Kruskal-Wallis tests did not reveal a significant group effect on the number of recovery steps for any perturbation or recovery step threshold (Fig. 2; Standard Threshold: $\mathrm{H}(3)=2.35, \mathrm{P}=0.5, \mathrm{H}(3)=1.2, \mathrm{P}=0.75$ and $\mathrm{H}(3)=2.66, \mathrm{P}=0.45$ for Pert1R, Pert2L and Pert9L, respectively; Individual Threshold: $H(3)=4.6, P=0.2, H(3)=2.68$, $P=0.44$ and $H(3)=0.36, P=0.95$ for Pert1 $R$, Pert2 $L$ and Pert9L, respectively). However, as can be seen in Figure 2, a large range of numbers of recovery steps was observed across the participants. 

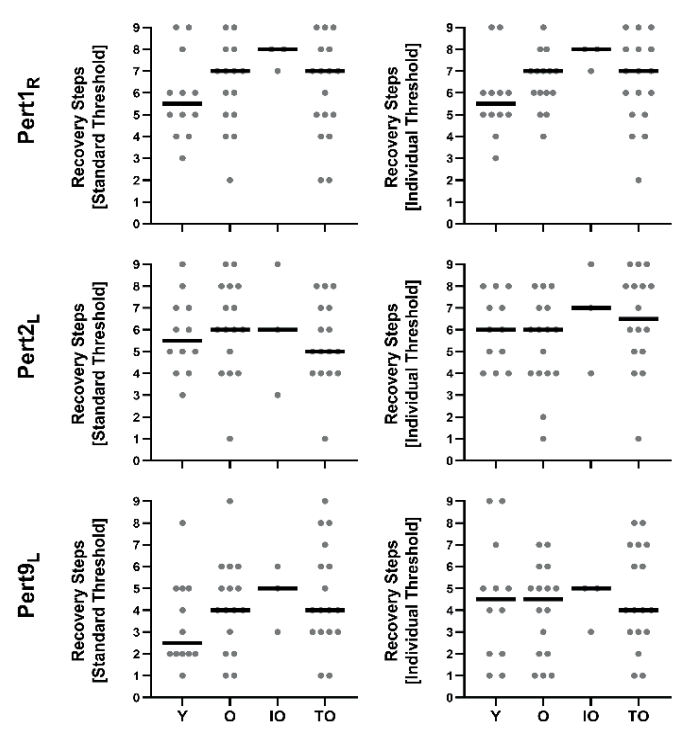

Figure 2: Group median and individual values for the number of recovery steps determined by the standard and individual thresholds for all the first perturbation to each leg (Pert $1_{R}$ and Pert2 $\mathrm{L}$ ) and the final left leg perturbation (Pert9 $\mathrm{L}$ ) for young adults $(\mathrm{Y})$, older adults with normal physical activity $(\mathrm{O})$, trained older adults $(\mathrm{TO})$ and physically impaired older adults (IO).

\section{No Associations between Type II Fibre Characteristics and Balance Recovery}

To test our hypothesis of a relationship between Type II muscle fibre characteristics (size and proportion) and stability recovery (number of recovery steps needed following a novel perturbation), Spearman correlations were conducted between these outcomes. These revealed no significant associations between the number of recovery steps following Pert1 $\mathrm{R}$ and Pert2L and the three Type II fibre characteristics: Type II fibre percentage, Type II fibre CSA and the percentage of total CSA taken up by Type II fibres (Table 2).

To provide further context for the muscle fibre characteristic associations, associations between the number of recovery steps and upper leg muscle volume and torque were also analysed. These Spearman correlations revealed no significant between upper leg muscle volume and the number of recovery steps following Pert1 $\mathrm{R}$ and Pert2L (Supplement Table 1). They revealed no significant associations between peak isometric and isokinetic knee extension and flexion torque and the 
number of recovery steps following Pert1R and one (of 8) moderate, significant association for Pert2L (significant for Peak Isometric Knee Flexion Torque; Supplement Table 1). Note that to provide further insight into these relationships, we also ran post hoc Spearman correlations between the fibre type characteristics and the muscle volume and torque outcomes, which revealed significant correlations between all pairs of muscle volume and torque outcomes, four (out of five) significant correlations between Type II fibre CSA and the muscle volume and torque outcomes but no significant correlations between Type II muscle fibre content or percentage of CSA from Type II fibres and the muscle volume and torque outcomes (Supplementary Table 2).

No Associations between Type II Fibre Characteristics and Adapted Balance Recovery

To explore the potential role of Type II muscle fibre characteristics in adaptability of stability recovery responses, associations between the recovery steps after eight repeated perturbations to the left leg (Pert9L) and the Type II muscle fibre characteristics were conducted. Spearman correlations revealed no significant associations between the number of recovery steps following Pert9L and the three Type II fibre characteristics: Type II fibre percentage, Type II fibre CSA and the percentage of total CSA taken up by Type II fibres (Table 2).

Again, to provide additional information, associations between the Pert9 $\mathrm{L}$ recovery steps and muscle volume and torque were also conducted. In general agreement with the fibre type correlations, Spearman correlations revealed no significant associations between upper leg muscle volume and the number of recovery steps following Pert9L, and only one (of 8) moderate, significant associations between peak isometric and isokinetic knee extension and flexion torque and the number of recovery steps following Pert9ı (Significant for Peak Isometric Knee Flexion Torque; Supplement Table 1). 
Table 2: Spearman Correlation Results for Recovery Steps and Type II Fibre Characteristics

\begin{tabular}{|c|c|c|c|c|c|c|c|}
\hline & & \multicolumn{2}{|c|}{ Pert1 $_{R}$} & \multicolumn{2}{|c|}{ Pert2 $\mathrm{L}$} & \multicolumn{2}{|c|}{ Pert9 ${ }_{L}$} \\
\hline & & $\begin{array}{c}\text { Standard } \\
\text { Threshol } \\
d\end{array}$ & $\begin{array}{c}\text { Individual } \\
\text { Threshol } \\
d\end{array}$ & $\begin{array}{c}\text { Standard } \\
\text { Threshol } \\
d\end{array}$ & $\begin{array}{c}\text { Individual } \\
\text { Threshol } \\
d\end{array}$ & $\begin{array}{c}\text { Standard } \\
\text { Threshol } \\
d\end{array}$ & $\begin{array}{c}\text { Individual } \\
\text { Threshol } \\
d\end{array}$ \\
\hline \multirow{3}{*}{$\begin{array}{c}\text { Type II } \\
\text { Fibre } \\
\text { Content [\%] }\end{array}$} & Spearman $r$ & 0.127 & 0.159 & 0.202 & -0.036 & 0.043 & -0.111 \\
\hline & $95 \% \mathrm{Cl}$ & $\begin{array}{c}-0.175 \text { to } \\
0.407\end{array}$ & $\begin{array}{c}-0.143 \text { to } \\
0.434\end{array}$ & $\begin{array}{c}-0.100 \text { to } \\
0.469\end{array}$ & $\begin{array}{c}-0.327 \text { to } \\
0.262\end{array}$ & $\begin{array}{c}-0.252 \text { to } \\
0.331\end{array}$ & $\begin{array}{c}-0.391 \text { to } \\
0.187\end{array}$ \\
\hline & $P$ & 0.395 & 0.286 & 0.174 & 0.812 & 0.771 & 0.452 \\
\hline \multirow{3}{*}{$\begin{array}{c}\text { Type II } \\
\text { Fibre Mean } \\
\text { CSA }\left[\mu \mathrm{m}^{2}\right]\end{array}$} & Spearman $r$ & -0.026 & -0.027 & 0.103 & 0.056 & -0.214 & -0.048 \\
\hline & $95 \% \mathrm{Cl}$ & $\begin{array}{c}-0.319 \text { to } \\
0.271\end{array}$ & $\begin{array}{c}-0.320 \text { to } \\
0.270\end{array}$ & $\begin{array}{c}-0.198 \text { to } \\
0.387\end{array}$ & $\begin{array}{c}-0.243 \text { to } \\
0.345\end{array}$ & $\begin{array}{c}-0.476 \text { to } \\
0.083\end{array}$ & $\begin{array}{c}-0.336 \text { to } \\
0.247\end{array}$ \\
\hline & $P$ & 0.861 & 0.856 & 0.490 & 0.710 & 0.144 & 0.745 \\
\hline \multirow{3}{*}{$\begin{array}{l}\text { CSA \% } \\
\text { from Type II } \\
\text { Fibres }\end{array}$} & Spearman $r$ & 0.143 & 0.120 & 0.194 & -0.125 & -0.085 & -0.161 \\
\hline & $95 \% \mathrm{Cl}$ & $\begin{array}{c}-0.159 \text { to } \\
0.420\end{array}$ & $\begin{array}{c}-0.182 \text { to } \\
0.401\end{array}$ & $\begin{array}{c}-0.107 \text { to } \\
0.463\end{array}$ & $\begin{array}{c}-0.405 \text { to } \\
0.177\end{array}$ & $\begin{array}{c}-0.367 \text { to } \\
0.213\end{array}$ & $\begin{array}{c}-0.433 \text { to } \\
0.137\end{array}$ \\
\hline & $P$ & 0.338 & 0.422 & 0.191 & 0.404 & 0.568 & 0.273 \\
\hline
\end{tabular}

\section{DISCUSSION}

In this study, we aimed to provide the first data on whether or not Type II muscle fibre characteristics associate with reactive balance during walking in young and older adults with varying muscle fibre type composition. We hypothesised that there would be a small to moderate relationship between Type II muscle fibre characteristics (size and proportion) and stability recovery (number of recovery steps needed following a novel perturbation). We conducted correlation analyses on our data from 48 young and older participants, with varying physical activity levels, muscle fibre type characteristics, muscle volume and strength, as well as stability recovery performance, and were not able to confirm this hypothesis, as we did not observe such correlations. The results of our secondary, exploratory correlations with muscle volume and strength generally aligned with the fibre type characteristic results, since associations between these parameters and stability recovery were also virtually absent. 
The current study found no significant differences in muscle fibre size in older adults compared to younger adults. Thus, we found no age-related differences between young and older participants who were not engaged in structured physical exercise but possessed comparable physical activity levels ( 10K steps/day) well above the general recommendation [60]. These results appear in contrast to previous studies reporting a substantial decrease in muscle fibre size, especially in Type II fibres, with age $[47,48,50]$. However, most of these studies did not measure and control for an age-related decrease in physical activity $[47,48,50]$, which could explain muscle fibre atrophy [56]. In line with our data, St-Jean-Pelletier et al. [49] observed an agerelated decrease in muscle Type II fibre size in sedentary middle-aged and older adults ( $\sim 7 \mathrm{~K}$ vs. $\sim 6.3 \mathrm{~K}$ steps/day on average) but not in physically active middleaged and older adults ( $12.9 \mathrm{~K}$ vs. $\sim 12.6 \mathrm{~K}$ steps/day). These results further support the hypothesis that muscle fibre atrophy is not simply a function of ageing per se but might also be driven by age-related physical inactivity and immobilization. However, it should be kept in mind that while the ANOVA was not significant, our post hoc analyses comparing the young adults with all older adults and with group $\mathrm{O}$ each found a significant effect of age on Type II fibre CSA (Hedges $g=0.82$ and $g=0.84$, respectively).

In the present study, the relative proportion of Type I muscle fibres was larger in endurance-trained older adults when compared to older adults with normal physical activity levels and young individuals. Although individual studies have shown conflicting findings regarding muscle fibre type distribution between young and older endurance-trained individuals [74,75], our results are in line with evidence indicating greater proportions of Type I fibres in endurance-trained older adults when compared to untrained older adults and untrained young individuals [54-56]. While evidence suggests long-term strength training provides a strong stimulus for the preservation of the structural and mechanical characteristics of skeletal muscle during ageing $[51,76]$, it remains unclear to what extent aerobic training alone can counteract these age-related changes $[74,77,78]$. However, endurance athletes may benefit from other adaptations that play a role in stability. One previous study reported that in a mixed group of older and younger adults, those who were recreational runners performed better on a forward falling task than the non-active participants, despite similar muscle strength values [28]. The authors suggested that 
runners frequently manage the stability of their body position during running and may therefore be better able to perform such destabilising tasks [28]. In our study, of the 17 participants in group TO, eight participated in regular running and a further two participated in badminton and tennis, which also involve multidirectional stepping and change of direction, and these individuals may have compensated their reduction in Type II fibre size and number with relatively more refined stability control.

The literature is currently mixed regarding the role of muscle strength in balance and falls $[9-13,17,37-40]$. Despite this, small to moderate associations between lower limb muscle strength and balance recovery performance following laboratory-based perturbations [26-36] led us to our hypothesis of a small to moderate relationship between Type II muscle fibre properties and balance recovery performance from a sudden walking perturbation. Our results suggest that the muscle fibre properties themselves are not critical factors for reactive balance recovery during walking, due to the lack of correlations, the inconsistent direction of the (non-significant) correlations, as well as the fact that the trained older group did not require a significantly different number of recovery steps, despite a significantly different proportion of the muscle fibre CSA coming from Type II fibres. In order to check if the younger adults' data were unduly influencing these results, we repeated the Spearmen correlation analyses post hoc with only the older participants included and these demonstrated similar results, with only one of the 18 associations being significant (Supplementary Table 3). Our collective muscle volume and strength correlation results were also mostly not statistically significant. These results may, to some extent, explain some of the mixed findings in the literature. Strength testing or training that primarily targets the muscle tissue properties and neglects other factors (such as motor coordination, response/movement speed, functionally relevant movements, among others; see Tieland et al. [44] and Hunter et al. [79] for reviews) may not capture critical elements of muscle function relevant for reactive balance control. That being said, the success of the recovery actions may not critically depend on maximal muscle strength at all. For example, one biomechanical modelling study found that, for backward balance loss, there is quite some overlap in the feasible regions for balance recovery during simulations with strength characteristics of young and older adults and recovery was only limited by strength 
during very short or very long recovery steps [80]. This is also supported by other studies demonstrating that older adults can improve their balance recovery responses to a level similar to young adults when repeatedly exposed to perturbations (acutely and over longer time periods), without changes in muscle strength [34,81]. As falls among community-dwelling older adults usually occur during dynamic movement [18-25], specificity of both strength and balance assessment and training should be key considerations in fall prevention.

\section{Study Limitations}

Some limitations of the current study should be considered. Regarding the walking perturbation protocol, our participants were informed that they would complete a walking balance challenge but were unaware of the specifics of the perturbations and no warnings or cues were given during the trial. However, beneficial effects of increased awareness of perturbations on stability recovery performance following trips have been shown $[82,83]$, even when the specifics are unknown. This may have resulted in better balance recovery performance than might be expected in natural settings. Regarding our outcome measure, the number of recovery steps, this is not a standard measure of balance recovery following perturbations to walking. However, no gold-standard outcome measure exists for this purpose. Other possibilities would be to use specific biomechanical variables such as the margin of stability or trunk lean at a specific time point. However, due to the heterogeneity in the balance recovery responses to such perturbations, using such specific measures at specific time points has limitations when trying to capture "overall" balance recovery during walking. We feel that the current measure gives a more general overall indicator that is more robust to individual differences in response behaviour. It should also be kept in mind that our muscle biopsies came from the vastus lateralis muscle which is not necessarily the most critical muscle for gait [2-4] (although it likely plays an important role in leg extension and energy absorption during the initial recovery steps) nor entirely representative of the fibre type properties from other lower limb muscles [84-86]. Finally, one potential limitation is the exploratory nature of some of the correlations and the lack of a priori correction for multiple comparisons. In general, despite the relatively high number of correlations performed, the lack of significance and the inconsistency in the direction of the 
correlations leads us to believe that our conclusions are justified and not dependent on spurious correlations (significant or not).

\section{Conclusions}

The current results indicate that Type II muscle fibre properties (proportion and dimensions) are not significantly associated with reactive balance recovery following perturbations to walking in young and older adults. Upper leg muscle volume and knee flexion and extension torque also failed to show a significant relationship with reactive balance recovery. These results have implications for muscle strength testing and training in fall prevention, as it appears that the muscle tissue properties are not key factors for balance recovery.

\section{Acknowledgements}

The authors thank Matthijs Hesselink for helpful discussions regarding the fibre type analysis, Tim Snijders for helpful comments and discussion on an earlier draft of the manuscript, Wouter Bijnens for assistance with the walking measurements, Paul Willems for technical support and Pascal Rense and Colin Dohmen for assistance with participant recruitment and various measurements. 


\section{Author Contributions}

CM: Conceptualisation, Data Curation, Formal Analysis, Investigation, Methodology, Visualisation, Writing - Original Draft, Writing - Reviewing \& Editing.

LG: Conceptualisation, Data Curation, Funding Acquisition, Investigation, Project Administration, Writing - Original Draft, Writing - Reviewing \& Editing.

GS: Investigation, Visualisation, Writing - Reviewing \& Editing.

EKM: Investigation, Writing - Reviewing \& Editing.

JAJ: Investigation, Writing - Reviewing \& Editing.

AG: Investigation, Software, Writing - Reviewing \& Editing.

KM: Conceptualisation, Resources, Supervision, Writing - Reviewing \& Editing.

$\mathrm{JH}$ : Conceptualisation, Funding Acquisition, Project Administration, Resources, Supervision, Writing - Reviewing \& Editing.

\section{Funding}

CM was financially supported by the NUTRIM Graduate Programme of Maastricht University Medical Centre ${ }^{+}$. LG and $\mathrm{JH}$ are financially supported by the TIFN research program Mitochondrial Health (ALWTF.2015.5). The project is partly organised by and executed under the auspices of TiFN, a public-private partnership on precompetitive research in food and nutrition.

\section{Competing Interests}

The authors declare no competing interests. Danone Nutricia Research, Friesland Campina, the Netherlands Organisation for Scientific Research and the Top-sector Agri\&Food are sponsors of the TIFN program and partly financed the project. They had no role in data collection and analysis, and the decision to publish. 
SUPPLEMENTARY MATERIAL FOR CHAPTER 6

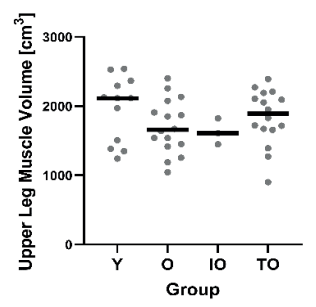

Supplement Fig. 1: Group median and individual upper leg muscle volume for young adults $(Y)$, older adults with normal physical activity $(\mathrm{O})$, trained older adults (TO) and physically impaired older adults (IO).
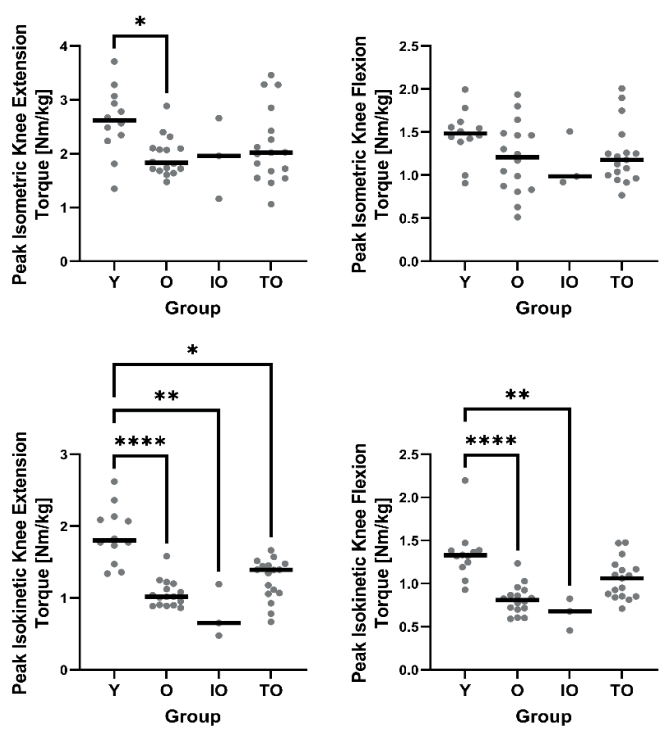

Supplement Figure 2: Group median and individual values for peak isometric and isokinetic knee extension and flexion torque for young adults $(\mathrm{Y})$, older adults with normal physical activity $(\mathrm{O})$, trained older adults $(\mathrm{TO})$ and physically impaired older adults (IO). *: $P<0.05^{* *} P<0.01{ }^{* * * *}: P<0.0001$ 
Supplement Table 1: Spearman correlation results for upper leg muscle volume, peak isometric and isokinetic knee extension and flexion torque and Pert $1_{R}$, Pert $2_{L}$ and Pert9 $9_{L}$

\begin{tabular}{|c|c|c|c|c|c|c|c|}
\hline & & \multicolumn{2}{|c|}{ Pert $_{R}$} & \multicolumn{2}{|c|}{ Pert2 $\mathrm{L}$} & \multicolumn{2}{|c|}{ Pert9 ${ }_{L}$} \\
\hline & & $\begin{array}{l}\text { Standard } \\
\text { Threshold }\end{array}$ & $\begin{array}{l}\text { Individual } \\
\text { Threshold }\end{array}$ & $\begin{array}{l}\text { Standard } \\
\text { Threshold }\end{array}$ & $\begin{array}{l}\text { Individual } \\
\text { Threshold }\end{array}$ & $\begin{array}{l}\text { Standard } \\
\text { Threshold }\end{array}$ & $\begin{array}{l}\text { Individual } \\
\text { Threshold }\end{array}$ \\
\hline & Spearman r & -0.1226 & -0.01128 & -0.01661 & -0.1461 & -0.1766 & -0.1825 \\
\hline \multirow[t]{3}{*}{$\begin{array}{l}\text { Upper Leg Muscle } \\
\text { Volume }\end{array}$} & $95 \% \mathrm{Cl}$ & $\begin{array}{c}-0.4061 \text { to } \\
0.1825\end{array}$ & $\begin{array}{c}-0.3086 \text { to } \\
0.2881\end{array}$ & $\begin{array}{c}-0.3134 \text { to } \\
0.2832\end{array}$ & $\begin{array}{c}-0.4259 \text { to } \\
0.1592\end{array}$ & $\begin{array}{c}-0.4484 \text { to } \\
0.1251\end{array}$ & $\begin{array}{c}-0.4532 \text { to } \\
0.1191\end{array}$ \\
\hline & $P$ & 0.417 & 0.9407 & 0.9127 & 0.3327 & 0.235 & 0.2195 \\
\hline & Spearman r & 0.1023 & 0.09993 & -0.06967 & -0.2268 & -0.03795 & -0.1397 \\
\hline \multirow[t]{3}{*}{$\begin{array}{l}\text { Peak Isometric Knee } \\
\text { Extension Torque }\end{array}$} & $95 \% \mathrm{Cl}$ & $\begin{array}{c}-0.1989 \text { to } \\
0.3858\end{array}$ & $\begin{array}{c}-0.2012 \text { to } \\
0.3838\end{array}$ & $\begin{array}{c}-0.3575 \text { to } \\
0.2302\end{array}$ & $\begin{array}{c}-0.4892 \text { to } \\
0.07325\end{array}$ & $\begin{array}{c}-0.3264 \text { to } \\
0.2570\end{array}$ & $\begin{array}{c}-0.4148 \text { to } \\
0.1589\end{array}$ \\
\hline & $P$ & 0.4939 & 0.5039 & 0.6417 & 0.1252 & 0.7979 & 0.3437 \\
\hline & Spearman r & -0.01427 & 0.002994 & -0.2043 & -0.3262 & -0.2399 & -0.3902 \\
\hline \multirow[t]{3}{*}{$\begin{array}{l}\text { Peak Isometric Knee } \\
\text { Flexion Torque }\end{array}$} & $95 \% \mathrm{Cl}$ & $\begin{array}{c}-0.3081 \text { to } \\
0.2821\end{array}$ & $\begin{array}{c}-0.2924 \text { to } \\
0.2979\end{array}$ & $\begin{array}{c}-0.4711 \text { to } \\
0.09667\end{array}$ & $\begin{array}{c}-0.5668 \text { to }- \\
0.03440\end{array}$ & $\begin{array}{c}-0.4971 \text { to } \\
0.05612\end{array}$ & $\begin{array}{c}-0.6125 \text { to }- \\
0.1108\end{array}$ \\
\hline & $P$ & 0.9242 & 0.9841 & 0.1683 & 0.0252 & 0.1006 & 0.0061 \\
\hline & Spearman r & -0.1622 & -0.2081 & -0.2041 & -0.06516 & -0.1998 & -0.1164 \\
\hline \multirow[t]{3}{*}{$\begin{array}{l}\text { Peak Isokinetic Knee } \\
\text { Extension Torque }\end{array}$} & $95 \% \mathrm{Cl}$ & $\begin{array}{c}-0.4365 \text { to } \\
0.1396\end{array}$ & $\begin{array}{l}-0.4741 \text { to } \\
0.09277\end{array}$ & $\begin{array}{l}-0.4709 \text { to } \\
0.09691\end{array}$ & $\begin{array}{c}-0.3535 \text { to } \\
0.2345\end{array}$ & $\begin{array}{c}-0.4647 \text { to } \\
0.09799\end{array}$ & $\begin{array}{c}-0.3950 \text { to } \\
0.1819\end{array}$ \\
\hline & $P$ & 0.276 & 0.1604 & 0.1688 & 0.6635 & 0.1734 & 0.4308 \\
\hline & Spearman r & -0.08 & -0.1216 & -0.2476 & -0.07478 & -0.1597 & -0.07469 \\
\hline \multirow[t]{2}{*}{$\begin{array}{l}\text { Peak Isokinetic Knee } \\
\text { Flexion Torque }\end{array}$} & $95 \% \mathrm{Cl}$ & $\begin{array}{c}-0.3665 \text { to } \\
0.2204\end{array}$ & $\begin{array}{c}-0.4023 \text { to } \\
0.1800\end{array}$ & $\begin{array}{l}-0.5058 \text { to } \\
0.05127\end{array}$ & $\begin{array}{c}-0.3620 \text { to } \\
0.2254\end{array}$ & $\begin{array}{c}-0.4317 \text { to } \\
0.1388\end{array}$ & $\begin{array}{c}-0.3589 \text { to } \\
0.2222\end{array}$ \\
\hline & $P$ & 0.593 & 0.4155 & 0.0933 & 0.6174 & 0.2781 & 0.6139 \\
\hline
\end{tabular}


Supplement Table 2: Spearman correlation matrix for upper leg muscle volume, peak isometric and isokinetic knee extension and flexion torque, Type II fibre content, Type II fibre mean CSA and CSA percentage from Type II fibres

\begin{tabular}{|c|c|c|c|c|c|c|c|c|c|}
\hline & & $\begin{array}{l}\text { Upper Leg } \\
\text { Muscle Volume }\end{array}$ & $\begin{array}{c}\text { Peak Isometric } \\
\text { Knee Extension } \\
\text { Torque } \\
\end{array}$ & $\begin{array}{c}\text { Peak Isometric } \\
\text { Knee Flexion } \\
\text { Torque }\end{array}$ & $\begin{array}{c}\text { Peak Isokinetic } \\
\text { Knee Extension } \\
\text { Torque }\end{array}$ & $\begin{array}{c}\text { Peak Isokinetic } \\
\text { Knee Flexion } \\
\text { Torque }\end{array}$ & $\begin{array}{l}\text { Type II Fibre } \\
\text { Content [\%] }\end{array}$ & $\begin{array}{c}\text { Type II Fibre } \\
\text { Mean CSA }\left[\mu \mathrm{m}^{2}\right]\end{array}$ & $\begin{array}{c}\text { CSA \% from Type } \\
\text { II Fibres }\end{array}$ \\
\hline \multirow[b]{2}{*}{$\begin{array}{c}\text { Upper Leg } \\
\text { Muscle Volume }\end{array}$} & Spearman $r$ & & & & & & & & \\
\hline & $\begin{array}{c}95 \% \mathrm{Cl} \\
\mathrm{P}\end{array}$ & & & & & & & & \\
\hline \multirow{2}{*}{$\begin{array}{c}\text { Peak Isometric } \\
\text { Knee Extension } \\
\text { Torque }\end{array}$} & Spearman $r$ & 0.405412 & & & & & & & \\
\hline & $\begin{array}{c}95 \% \mathrm{Cl} \\
\mathrm{P}\end{array}$ & $\begin{array}{c}0.1252 \text { to } 0.6257 \\
\mathbf{0 . 0 0 4 7}\end{array}$ & & & & & & & \\
\hline \multirow{3}{*}{$\begin{array}{l}\text { Peak Isometric } \\
\text { Knee Flexion } \\
\text { Torque }\end{array}$} & Spearman $r$ & 0.533534 & 0.646 & & & & & & \\
\hline & $95 \% \mathrm{Cl}$ & 0.2829 to 0.7159 & 0.4361 to 0.7891 & & & & & & \\
\hline & $P$ & 0.0001 & $<0.0001$ & & & & & & \\
\hline \multirow{3}{*}{$\begin{array}{c}\text { Peak Isokinetic } \\
\text { Knee Extension } \\
\text { Torque }\end{array}$} & Spearman $r$ & 0.631707 & 0.608 & 0.528 & & & & & \\
\hline & $95 \% \mathrm{Cl}$ & 0.4137 to 0.7812 & 0.3839 to 0.7642 & 0.2790 to 0.7105 & & & & & \\
\hline & $P$ & $<0.0001$ & $<0.0001$ & 0.0001 & & & & & \\
\hline \multirow{3}{*}{$\begin{array}{l}\text { Peak Isokinetic } \\
\text { Knee Flexion } \\
\text { Torque }\end{array}$} & Spearman $r$ & 0.490634 & 0.670 & 0.538 & 0.877 & & & & \\
\hline & $95 \% \mathrm{Cl}$ & 0.2286 to 0.6864 & 0.4697 to 0.8045 & 0.2914 to 0.7171 & 0.7858 to 0.9305 & & & & \\
\hline & $P$ & 0.0005 & $<0.0001$ & 0.0001 & $<0.0001$ & & & & \\
\hline \multirow{3}{*}{$\begin{array}{l}\text { Type II Fibre } \\
\text { Content [\%] }\end{array}$} & Spearman $r$ & -0.04845 & 0.043 & 0.175 & 0.045 & -0.118 & & & \\
\hline & $95 \% \mathrm{Cl}$ & -0.3388 to 0.2503 & -0.2523 to 0.3308 & -0.1234 to 0.4443 & -0.2508 to 0.3322 & -0.3961 to 0.1806 & & & \\
\hline & $P$ & 0.7464 & 0.7723 & 0.2342 & 0.7639 & 0.4257 & & & \\
\hline \multirow{3}{*}{$\begin{array}{c}\text { Type II Fibre } \\
\text { Mean CSA } \\
{\left[\mu \mathrm{m}^{2}\right]}\end{array}$} & Spearman $r$ & 0.570421 & 0.282 & 0.297 & 0.564 & 0.405 & -0.018 & & \\
\hline & $95 \% \mathrm{Cl}$ & 0.3310 to 0.7408 & -0.01117 to 0.5302 & $\begin{array}{c}0.005062 \text { to } \\
0.5418\end{array}$ & 0.3256 to 0.7350 & 0.1284 to 0.6235 & -0.2946 to 0.2610 & & \\
\hline & $P$ & $<0.0001$ & 0.0523 & 0.0406 & $<0.0001$ & 0.0043 & 0.8970 & & \\
\hline \multirow{3}{*}{$\begin{array}{l}\text { CSA \% from } \\
\text { Type II Fibres }\end{array}$} & Spearman $r$ & 0.066374 & 0.187 & 0.297 & 0.185 & 0.048 & 0.855 & 0.331 & \\
\hline & $95 \% \mathrm{Cl}$ & -0.2334 to 0.3546 & -0.1114 to 0.4540 & $\begin{array}{c}0.005538 \text { to } \\
0.5421\end{array}$ & -0.1132 to 0.4526 & -0.2473 to 0.3355 & 0.7566 to 0.9153 & 0.05832 to 0.5575 & \\
\hline & $P$ & 0.6576 & 0.2038 & 0.0403 & 0.2082 & 0.7449 & $<0.0001$ & 0.0155 & \\
\hline
\end{tabular}


Supplementary Table 3: Spearman Correlation Results for Recovery Steps and Type II Fibre Characteristics in the Older Participants.

\begin{tabular}{|c|c|c|c|c|c|c|c|}
\hline & & \multicolumn{2}{|c|}{ Pert1 $_{R}$} & \multicolumn{2}{|c|}{ Pert2 $L_{L}$} & \multicolumn{2}{|c|}{ Pert9 ${ }_{\mathrm{L}}$} \\
\hline & & $\begin{array}{l}\text { Standard } \\
\text { Threshold }\end{array}$ & $\begin{array}{l}\text { Individual } \\
\text { Threshold }\end{array}$ & $\begin{array}{l}\text { Standard } \\
\text { Threshold }\end{array}$ & $\begin{array}{l}\text { Individual } \\
\text { Threshold }\end{array}$ & $\begin{array}{l}\text { Standard } \\
\text { Threshold }\end{array}$ & $\begin{array}{l}\text { Individual } \\
\text { Threshold }\end{array}$ \\
\hline \multirow{3}{*}{$\begin{array}{l}\text { Type II Fibre } \\
\text { Content [\%] }\end{array}$} & Spearman $r$ & 0.2188 & 0.3559 & 0.1725 & -0.1154 & -0.08924 & -0.2365 \\
\hline & $95 \% \mathrm{Cl}$ & $\begin{array}{c}-0.1335 \text { to } \\
0.5220\end{array}$ & $\begin{array}{c}0.01544 \text { to } \\
0.6224\end{array}$ & $\begin{array}{c}-0.1805 \text { to } \\
0.4861\end{array}$ & $\begin{array}{c}-0.4404 \text { to } \\
0.2362\end{array}$ & $\begin{array}{c}-0.4143 \text { to } \\
0.2560\end{array}$ & $\begin{array}{c}-0.5315 \text { to } \\
0.1098\end{array}$ \\
\hline & $P$ & 0.2066 & 0.0359 & 0.3217 & 0.5090 & 0.6048 & 0.1650 \\
\hline \multirow{3}{*}{$\begin{array}{c}\text { Type II Fibre } \\
\text { Mean CSA } \\
{\left[\mu \mathrm{m}^{2}\right]}\end{array}$} & Spearman $r$ & 0.008247 & 0.001569 & 0.06739 & -0.009371 & -0.2108 & -0.08795 \\
\hline & $95 \% \mathrm{Cl}$ & $\begin{array}{c}-0.3350 \text { to } \\
0.3496\end{array}$ & $\begin{array}{c}-0.3409 \text { to } \\
0.3437\end{array}$ & $\begin{array}{c}-0.2814 \text { to } \\
0.4005\end{array}$ & $\begin{array}{c}-0.3506 \text { to } \\
0.3340\end{array}$ & $\begin{array}{c}-0.5119 \text { to } \\
0.1364\end{array}$ & $\begin{array}{c}-0.4132 \text { to } \\
0.2572\end{array}$ \\
\hline & $P$ & 0.9625 & 0.9929 & 0.7005 & 0.9574 & 0.2172 & 0.6100 \\
\hline \multirow{3}{*}{$\begin{array}{l}\text { CSA \% from } \\
\text { Type II Fibres }\end{array}$} & Spearman $r$ & 0.2514 & 0.3135 & 0.1707 & -0.2258 & -0.1783 & -0.3070 \\
\hline & $95 \% \mathrm{Cl}$ & $\begin{array}{c}-0.09948 \text { to } \\
0.5467\end{array}$ & $\begin{array}{c}-0.03227 \text { to } \\
0.5923\end{array}$ & $\begin{array}{c}-0.1823 \text { to } \\
0.4847\end{array}$ & $\begin{array}{c}-0.5273 \text { to } \\
0.1263\end{array}$ & $\begin{array}{c}-0.4866 \text { to } \\
0.1693\end{array}$ & $\begin{array}{c}-0.5840 \text { to } \\
0.03403\end{array}$ \\
\hline & $P$ & 0.1452 & 0.0667 & 0.3269 & 0.1922 & 0.2980 & 0.0686 \\
\hline
\end{tabular}




\section{REFERENCES}

1. Janssen I, Heymsfield SB, Wang Z, Ross R (2000) Skeletal muscle mass and distribution in 468 men and women aged 18-88 yr. J Appl Physiol 89 (1):81-88. doi:10.1152/jappl.2000.89.1.81

2. Kulmala JP, Korhonen MT, Ruggiero L, Kuitunen S, Suominen H, Heinonen A, Mikkola A, Avela J (2016) Walking and Running Require Greater Effort from Ankle than Knee Extensor Muscles. Med Sci Sports Exerc. doi:10.1249/mss.0000000000001020

3. Kulmala JP, Korhonen MT, Kuitunen S, Suominen H, Heinonen A, Mikkola A, Avela J (2014) Which muscles compromise human locomotor performance with age? Journal of the Royal Society, Interface / the Royal Society 11 (100):20140858. doi:10.1098/rsif.2014.0858

4. Pandy MG, Andriacchi TP (2010) Muscle and joint function in human locomotion. Annu Rev Biomed Eng 12:401-433. doi:10.1146/annurev-bioeng-070909-105259

5. McCrum C, Leow P, Epro G, Konig M, Meijer K, Karamanidis K (2018) Alterations in Leg Extensor Muscle-Tendon Unit Biomechanical Properties With Ageing and Mechanical Loading. Front Physiol 9 (150):150. doi:10.3389/fphys.2018.00150

6. Stenroth L, Peltonen J, Cronin NJ, Sipila S, Finni T (2012) Age-related differences in Achilles tendon properties and triceps surae muscle architecture in vivo. Journal of applied physiology (Bethesda, Md : 1985) 113 (10):1537-1544. doi:10.1152/japplphysiol.00782.2012 7. Onambele GL, Narici MV, Maganaris CN (2006) Calf muscle-tendon properties and postural balance in old age. Journal of applied physiology (Bethesda, Md : 1985) 100 (6):2048-2056. doi:10.1152/japplphysiol.01442.2005

8. Mademli L, Arampatzis A, Karamanidis K (2008) Dynamic stability control in forward falls: postural corrections after muscle fatigue in young and older adults. Eur J Appl Physiol 103 (3):295-306. doi:10.1007/s00421-008-0704-z

9. De Rekeneire N, Visser M, Peila R, Nevitt MC, Cauley JA, Tylavsky FA, Simonsick EM, Harris TB (2003) Is a Fall Just a Fall: Correlates of Falling in Healthy Older Persons. The Health, Aging and Body Composition Study. J Am Geriatr Soc 51 (6):841-846. doi:10.1046/j.1365-2389.2003.51267.x

10. Cattagni T, Scaglioni G, Laroche D, Van Hoecke J, Gremeaux V, Martin A (2014) Ankle muscle strength discriminates fallers from non-fallers. Front Aging Neurosci 6:336. doi:10.3389/fnagi.2014.00336

11. Perry MC, Carville SF, Smith ICH, Rutherford OM, Newham DJ (2007) Strength, power output and symmetry of leg muscles: effect of age and history of falling. Eur $\mathrm{J}$ Appl Physiol 100 (5):553-561. doi:10.1007/s00421-006-0247-0

12. LaRoche DP, Cremin KA, Greenleaf B, Croce RV (2010) Rapid torque development in older female fallers and nonfallers: A comparison across lower-extremity muscles. J Electromyogr Kinesiol 20 (3):482-488. doi:10.1016/j.jelekin.2009.08.004

13. Kamo T, Asahi R, Azami M, Ogihara H, Ikeda T, Suzuki K, Nishida Y (2019) Rate of torque development and the risk of falls among community dwelling older adults in Japan. Gait Posture 72:28-33. doi:10.1016/j.gaitpost.2019.05.019

14. Skelton DA, Kennedy J, Rutherford OM (2002) Explosive power and asymmetry in leg muscle function in frequent fallers and non-fallers aged over 65. Age Ageing 31 (2):119-125. doi:10.1093/ageing/31.2.119

15. Gadelha AB, Neri SGR, Nobrega OT, Pereira JC, Bottaro M, Fonseca A, Lima RM (2018) Muscle quality is associated with dynamic balance, fear of falling, and falls in older women. Exp Gerontol 104:1-6. doi:10.1016/j.exger.2018.01.003 
16. Gadelha AB, Neri SGR, Bottaro M, Lima RM (2018) The relationship between muscle quality and incidence of falls in older community-dwelling women: An 18-month follow-up study. Exp Gerontol 110:241-246. doi:10.1016/j.exger.2018.06.018

17. Melzer I, Benjuya N, Kaplanski J (2004) Postural stability in the elderly: a comparison between fallers and non-fallers. Age Ageing 33 (6):602-607. doi:10.1093/ageing/afh218

18. Berg WP, Alessio HM, Mills EM, Tong C (1997) Circumstances and consequences of falls in independent community-dwelling older adults. Age Ageing 26 (4):261-268. doi:10.1093/ageing/26.4.261

19. Tinetti ME, Speechley M, Ginter SF (1988) Risk factors for falls among elderly persons living in the community. N Engl J Med 319 (26):1701-1707. doi:10.1056/NEJM198812293192604

20. Sheldon JH (1960) On the Natural History of Falls in Old Age. Br Med J 2 (5214):16851690. doi:10.1136/bmj.2.5214.1685

21. Lord SR, Ward JA, Williams P, Anstey KJ (1993) An epidemiological study of falls in older community-dwelling women: the Randwick falls and fractures study. Aust $\mathrm{J}$ Public Health 17 (3):240-245

22. Niino N, Tsuzuku S, Ando F, Shimokata H (2000) Frequencies and circumstances of falls in the National Institute for Longevity Sciences, Longitudinal Study of Aging (NILS-LSA). J Epidemiol 10 (1 Suppl):S90-94. doi:10.2188/jea.10.1sup_90

23. Talbot LA, Musiol RJ, Witham EK, Metter EJ (2005) Falls in young, middle-aged and older community dwelling adults: perceived cause, environmental factors and injury. BMC Public Health 5:86. doi:10.1186/1471-2458-5-86

24. Crenshaw JR, Bernhardt KA, Achenbach SJ, Atkinson EJ, Khosla S, Kaufman KR, Amin $S$ (2017) The circumstances, orientations, and impact locations of falls in communitydwelling older women. Arch Gerontol Geriatr 73:240-247. doi:10.1016/j.archger.2017.07.011 25. McCrum C (2019) A Trip to Remember: Assessing and Improving Walking Stability in Older Adults. Maastricht University, Gildeprint Drukkerijen

26. Grabiner MD, Owings TM, Pavol MJ (2005) Lower extremity strength plays only a small role in determining the maximum recoverable lean angle in older adults. J Gerontol A Biol Sci Med Sci 60 (11):1447-1450. doi:10.1093/gerona/60.11.1447

27. Karamanidis K, Arampatzis A, Mademli L (2008) Age-related deficit in dynamic stability control after forward falls is affected by muscle strength and tendon stiffness. J Electromyogr Kinesiol 18 (6):980-989. doi:10.1016/j.jelekin.2007.04.003

28. Karamanidis K, Arampatzis A (2007) Age-related degeneration in leg-extensor muscletendon units decreases recovery performance after a forward fall: compensation with running experience. Eur J Appl Physiol 99 (1):73-85. doi:10.1007/s00421-006-0318-2

29. Carty CP, Barrett RS, Cronin NJ, Lichtwark GA, Mills PM (2012) Lower limb muscle weakness predicts use of a multiple- versus single-step strategy to recover from forward loss of balance in older adults. J Gerontol A Biol Sci Med Sci 67 (11):1246-1252. doi:10.1093/gerona/gls149

30. Carty CP, Cronin NJ, Lichtwark GA, Mills PM, Barrett RS (2012) Lower limb muscle moments and power during recovery from forward loss of balance in male and female single and multiple steppers. Clin Biomech (Bristol, Avon) 27 (10):1031-1037. doi:10.1016/j.clinbiomech.2012.07.009

31. Pavol MJ, Owings TM, Foley KT, Grabiner MD (2002) Influence of lower extremity strength of healthy older adults on the outcome of an induced trip. J Am Geriatr Soc 50 (2):256-262 
32. Pijnappels M, van der Burg PJ, Reeves ND, van Dieen JH (2008) Identification of elderly fallers by muscle strength measures. Eur J Appl Physiol 102 (5):585-592. doi:10.1007/s00421-007-0613-6

33. Pijnappels M, Bobbert MF, van Dieen JH (2005) Push-off reactions in recovery after tripping discriminate young subjects, older non-fallers and older fallers. Gait Posture 21 (4):388-394. doi:10.1016/j.gaitpost.2004.04.009

34. Epro G, McCrum C, Mierau A, Leyendecker M, Brüggemann GP, Karamanidis K (2018) Effects of triceps surae muscle strength and tendon stiffness on the reactive dynamic stability and adaptability of older female adults during perturbed walking. Journal of applied physiology (Bethesda, Md : 1985) 124 (6):1541-1549. doi:10.1152/japplphysiol.00545.2017 35. Ding L, Yang F (2016) Muscle weakness is related to slip-initiated falls among $\begin{array}{llllll}\text { community-dwelling older } \quad \text { adults. } & J & \text { Biomech } & 49 & \text { (2):238-243. }\end{array}$ doi:10.1016/j.jbiomech.2015.12.009

36. Han L, Yang F (2015) Strength or power, which is more important to prevent slip-related falls? Hum Mov Sci 44:192-200. doi:10.1016/j.humov.2015.09.001

37. Orr R, Raymond J, Fiatarone Singh M (2008) Efficacy of progressive resistance training on balance performance in older adults : a systematic review of randomized controlled trials. Sports Med 38 (4):317-343. doi:10.2165/00007256-200838040-00004

38. Howe TE, Rochester L, Neil F, Skelton DA, Ballinger C (2011) Exercise for improving balance in older people. Cochrane Database Syst Rev (11):Cd004963. doi:10.1002/14651858.CD004963.pub3

39. Horlings CG, van Engelen BG, Allum JH, Bloem BR (2008) A weak balance: the contribution of muscle weakness to postural instability and falls. Nat Clin Pract Neurol 4 (9):504-515. doi:10.1038/ncpneuro0886

40. Sherrington C, Fairhall NJ, Wallbank GK, Tiedemann A, Michaleff ZA, Howard K, Clemson L, Hopewell S, Lamb SE (2019) Exercise for preventing falls in older people living in the community. Cochrane Database Syst Rev 1:CD012424. doi:10.1002/14651858.CD012424.pub2

41. Orr R (2010) Contribution of muscle weakness to postural instability in the elderly. A systematic review. Eur J Phys Rehabil Med 46 (2):183-220

42. Wilkinson DJ, Piasecki M, Atherton PJ (2018) The age-related loss of skeletal muscle mass and function: Measurement and physiology of muscle fibre atrophy and muscle fibre loss in humans. Ageing research reviews 47:123-132. doi:10.1016/j.arr.2018.07.005

43. Deschenes MR (2004) Effects of Aging on Muscle Fibre Type and Size. Sports Med 34 (12):809-824. doi:10.2165/00007256-200434120-00002

44. Tieland M, Trouwborst I, Clark BC (2018) Skeletal muscle performance and ageing. Journal of cachexia, sarcopenia and muscle 9 (1):3-19. doi:10.1002/jcsm.12238

45. Mitchell WK, Atherton PJ, Williams J, Larvin M, Lund JN, Narici M (2012) Sarcopenia, Dynapenia, and the Impact of Advancing Age on Human Skeletal Muscle Size and Strength; a Quantitative Review. Front Physiol 3. doi:10.3389/fphys.2012.00260

46. Kramer IF, Snijders T, Smeets JSJ, Leenders M, van Kranenburg J, den Hoed M, Verdijk LB, Poeze M, van Loon LJC (2017) Extensive Type II Muscle Fiber Atrophy in Elderly Female Hip Fracture Patients. The Journals of Gerontology: Series A 72 (10):1369-1375. doi:10.1093/gerona/glw253

47. McPhee JS, Cameron J, Maden-Wilkinson T, Piasecki M, Yap MH, Jones DA, Degens H (2018) The Contributions of Fiber Atrophy, Fiber Loss, In Situ Specific Force, and Voluntary Activation to Weakness in Sarcopenia. The Journals of Gerontology: Series A 73 (10):12871294. doi:10.1093/gerona/gly040 
48. Nilwik R, Snijders T, Leenders M, Groen BBL, van Kranenburg J, Verdijk LB, van Loon LJC (2013) The decline in skeletal muscle mass with aging is mainly attributed to a reduction in type II muscle fiber size. Exp Gerontol 48 (5):492-498. doi:10.1016/j.exger.2013.02.012 49. St-Jean-Pelletier F, Pion CH, Leduc-Gaudet J-P, Sgarioto N, Zovilé I, Barbat-Artigas S, Reynaud O, Alkaterji F, Lemieux FC, Grenon A, Gaudreau P, Hepple RT, Chevalier S, Belanger M, Morais JA, Aubertin-Leheudre M, Gouspillou G (2017) The impact of ageing, physical activity, and pre-frailty on skeletal muscle phenotype, mitochondrial content, and intramyocellular lipids in men. Journal of cachexia, sarcopenia and muscle 8 (2):213-228. doi:10.1002/jcsm.12139

50. Lee W-S, Cheung W-H, Qin L, Tang N, Leung K-S (2006) Age-associated Decrease of Type IIA/B Human Skeletal Muscle Fibers. Clinical Orthopaedics and Related Research $®$ 450:231-237. doi:10.1097/01.blo.0000218757.97063.21

51. Klitgaard H, Mantoni M, Schiaffino S, Ausoni S, Gorza L, Laurent-Winter C, Schnohr P, Saltin B (1990) Function, morphology and protein expression of ageing skeletal muscle: a cross-sectional study of elderly men with different training backgrounds. Acta Physiol Scand 140 (1):41-54. doi:10.1111/j.1748-1716.1990.tb08974.x

52. Hameed M, Orrell RW, Cobbold M, Goldspink G, Harridge SD (2003) Expression of IGFI splice variants in young and old human skeletal muscle after high resistance exercise. J Physiol 547 (Pt 1):247-254. doi:10.1113/jphysiol.2002.032136

53. Short KR, Vittone JL, Bigelow ML, Proctor DN, Coenen-Schimke JM, Rys P, Nair KS (2005) Changes in myosin heavy chain mRNA and protein expression in human skeletal muscle with age and endurance exercise training. Journal of applied physiology (Bethesda, Md : 1985) 99 (1):95-102. doi:10.1152/japplphysiol.00129.2005

54. Aagaard P, Magnusson PS, Larsson B, Kjaer M, Krustrup P (2007) Mechanical muscle function, morphology, and fiber type in lifelong trained elderly. Med Sci Sports Exerc 39 (11):1989-1996. doi:10.1249/mss.0b013e31814fb402

55. Mackey AL, Karlsen A, Couppé C, Mikkelsen UR, Nielsen RH, Magnusson SP, Kjaer M (2014) Differential satellite cell density of type I and II fibres with lifelong endurance running in old men. Acta Physiol (Oxf) 210 (3):612-627. doi:10.1111/apha.12195

56. Mosole S, Carraro U, Kern H, Loefler S, Fruhmann H, Vogelauer M, Burggraf S, Mayr W, Krenn M, Paternostro-Sluga T, Hamar D, Cvecka J, Sedliak M, Tirpakova V, Sarabon N, Musarò A, Sandri M, Protasi F, Nori A, Pond A, Zampieri S (2014) Long-term high-level exercise promotes muscle reinnervation with age. J Neuropathol Exp Neurol 73 (4):284-294. doi:10.1097/nen.0000000000000032

57. Guralnik JM, Simonsick EM, Ferrucci L, Glynn RJ, Berkman LF, Blazer DG, Scherr PA, Wallace RB (1994) A short physical performance battery assessing lower extremity function: association with self-reported disability and prediction of mortality and nursing home admission. J Gerontol 49 (2):M85-94. doi:10.1093/geronj/49.2.m85

58. Dempster P, Aitkens S (1995) A new air displacement method for the determination of human body composition. Med Sci Sports Exerc 27 (12):1692-1697

59. van der Berg JD, Willems PJB, van der Velde JHPM, Savelberg HHCM, Schaper NC, Schram MT, Sep SJS, Dagnelie PC, Bosma H, Stehouwer CDA, Koster A (2016) Identifying waking time in 24-h accelerometry data in adults using an automated algorithm. J Sports Sci 34 (19):1867-1873. doi:10.1080/02640414.2016.1140908

60. Tudor-Locke C, Craig CL, Brown WJ, Clemes SA, De Cocker K, Giles-Corti B, Hatano Y, Inoue S, Matsudo SM, Mutrie N, Oppert JM, Rowe DA, Schmidt MD, Schofield GM, Spence JC, Teixeira PJ, Tully MA, Blair SN (2011) How many steps/day are enough? For adults. Int J Behav Nutr Phys Act 8 (1):79. doi:10.1186/1479-5868-8-79 
61. Bergström J, Hermansen L, Hultman E, Saltin B (1967) Diet, Muscle Glycogen and Physical Performance. Acta Physiol Scand 71 (2-3):140-150. doi:10.1111/j.17481716.1967.tb03720.x

62. Koopman R, Schaart G, Hesselink MK (2001) Optimisation of oil red O staining permits combination with immunofluorescence and automated quantification of lipids. Histochem Cell Biol 116 (1):63-68. doi:10.1007/s004180100297

63. Schneider CA, Rasband WS, Eliceiri KW (2012) NIH Image to ImageJ: 25 years of image analysis. Nat Methods 9 (7):671-675. doi:10.1038/nmeth.2089

64. Hof AL, Gazendam MG, Sinke WE (2005) The condition for dynamic stability. J Biomech 38 (1):1-8. doi:10.1016/j.jbiomech.2004.03.025

65. McCrum C, Willems P, Karamanidis K, Meijer K (2019) Stability-normalised walking speed: A new approach for human gait perturbation research. J Biomech 87:48-53. doi:10.1016/j.jbiomech.2019.02.016

66. McCrum C, Karamanidis K, Grevendonk L, Zijlstra W, Meijer K (2020) Older adults demonstrate interlimb transfer of reactive gait adaptations to repeated unpredictable gait perturbations. Geroscience 42 (1):39-49. doi:10.1007/s11357-019-00130-x

67. McCrum C, Karamanidis K, Willems P, Zijlstra W, Meijer K (2018) Retention, savings and interlimb transfer of reactive gait adaptations in humans following unexpected perturbations. Commun Biol 1 (1):230. doi:10.1038/s42003-018-0238-9

68. Zeni JA, Jr., Richards JG, Higginson JS (2008) Two simple methods for determining gait events during treadmill and overground walking using kinematic data. Gait Posture 27 (4):710-714. doi:10.1016/j.gaitpost.2007.07.007

69. McCrum C, Lucieer F, van de Berg R, Willems P, Perez Fornos A, Guinand N, Karamanidis K, Kingma H, Meijer K (2019) The walking speed-dependency of gait variability in bilateral vestibulopathy and its association with clinical tests of vestibular function. Sci Rep 9 (1):18392. doi:10.1038/s41598-019-54605-0

70. Süptitz F, Moreno Catala M, Brüggemann GP, Karamanidis K (2013) Dynamic stability control during perturbed walking can be assessed by a reduced kinematic model across the adult female lifespan. Hum Mov Sci 32 (6):1404-1414. doi:10.1016/j.humov.2013.07.008

71. Suptitz F, Karamanidis K, Moreno Catala M, Bruggemann GP (2012) Symmetry and reproducibility of the components of dynamic stability in young adults at different walking velocities on the treadmill. J Electromyogr Kinesiol 22 (2):301-307. doi:10.1016/j.jelekin.2011.12.007

72. Cohen J (1992) A power primer. Psychol Bull 112 (1):155-159. doi:10.1037//00332909.112.1.155

73. Brydges CR (2019) Effect Size Guidelines, Sample Size Calculations, and Statistical Power in Gerontology. Innov Aging 3 (4):igz036. doi:10.1093/geroni/igz036

74. McKendry J, Breen L, Shad BJ, Greig CA (2018) Muscle morphology and performance in master athletes: A systematic review and meta-analyses. Ageing research reviews 45:62-82. doi:10.1016/j.arr.2018.04.007

75. Zampieri S, Pietrangelo L, Loefler S, Fruhmann H, Vogelauer M, Burggraf S, Pond A, Grim-Stieger M, Cvecka J, Sedliak M, Tirpáková V, Mayr W, Sarabon N, Rossini K, Barberi L, De Rossi M, Romanello V, Boncompagni S, Musarò A, Sandri M, Protasi F, Carraro U, Kern H (2015) Lifelong physical exercise delays age-associated skeletal muscle decline. J Gerontol A Biol Sci Med Sci 70 (2):163-173. doi:10.1093/gerona/glu006

76. Pearson SJ, Young A, Macaluso A, Devito G, Nimmo MA, Cobbold M, Harridge SD (2002) Muscle function in elite master weightlifters. Med Sci Sports Exerc 34 (7):1199-1206. doi:10.1097/00005768-200207000-00023 
77. Coggan AR, Spina RJ, King DS, Rogers MA, Brown M, Nemeth PM, Holloszy JO (1992) Skeletal muscle adaptations to endurance training in 60- to 70-yr-old men and women. Journal of applied physiology (Bethesda, Md : 1985) 72 (5):1780-1786. doi:10.1152/jappl.1992.72.5.1780

78. Cadore EL, Pinto RS, Bottaro M, Izquierdo M (2014) Strength and endurance training prescription in healthy and frail elderly. Aging Dis 5 (3):183-195. doi:10.14336/ad.2014.0500183

79. Hunter SK, Pereira HM, Keenan KG (2016) The aging neuromuscular system and motor performance. Journal of applied physiology (Bethesda, Md : 1985) 121 (4):982-995. doi:10.1152/japplphysiol.00475.2016

80. Kadono N, Pavol MJ (2013) Effects of aging-related losses in strength on the ability to recover from a backward balance loss. J Biomech 46 (1):13-18. doi:10.1016/j.jbiomech.2012.08.046

81. Epro G, Mierau A, McCrum C, Leyendecker M, Bruggemann GP, Karamanidis K (2018) Retention of gait stability improvements over 1.5 years in older adults: effects of perturbation exposure and triceps surae neuromuscular exercise. J Neurophysiol 119 (6):2229-2240. doi:10.1152/jn.00513.2017

82. Oludare SO, Pater ML, Rosenblatt NJ, Grabiner MD (2018) Trip-specific training enhances recovery after large postural disturbances for which there is NO expectation. Gait Posture 61:382-386. doi:10.1016/j.gaitpost.2018.02.001

83. Pater ML, Rosenblatt NJ, Grabiner MD (2015) Expectation of an upcoming large postural perturbation influences the recovery stepping response and outcome. Gait Posture 41 (1):335-337. doi:10.1016/j.gaitpost.2014.10.026

84. Luden N, Minchev K, Hayes E, Louis E, Trappe T, Trappe S (2008) Human vastus lateralis and soleus muscles display divergent cellular contractile properties. American journal of physiology Regulatory, integrative and comparative physiology 295 (5):R1593R1598. doi:10.1152/ajpregu.90564.2008

85. Gollnick PD, Sjödin B, Karlsson J, Jansson E, Saltin B (1974) Human soleus muscle: a comparison of fiber composition and enzyme activities with other leg muscles. Pflugers Arch 348 (3):247-255. doi:10.1007/bf00587415

86. Edgerton VR, Smith JL, Simpson DR (1975) Muscle fibre type populations of human leg muscles. Histochem J 7 (3):259-266. doi:10.1007/bf01003594 


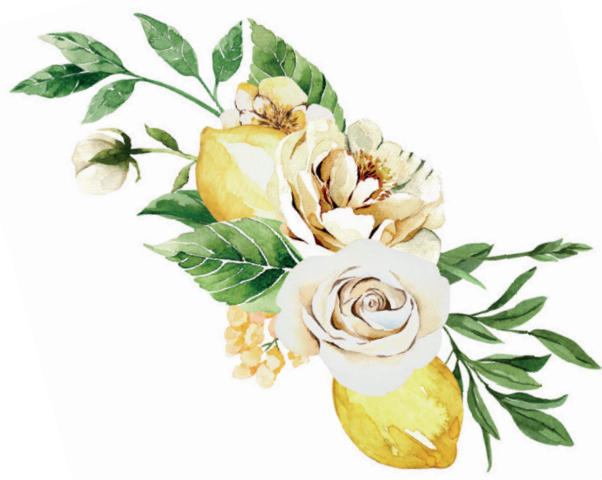

\section{CHAPTER 7}

General discussion

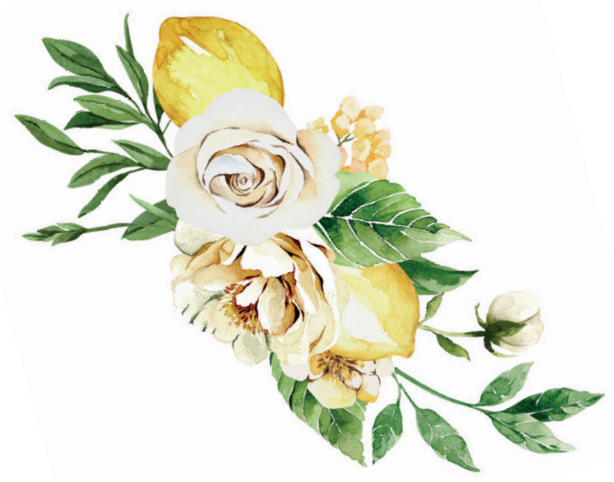


Although advanced chronological age is one of the most critical risk factors for many chronic diseases, the rate at which physiological functions and overall health status decline with age - defined as biological age - is highly variable among individuals (1). In contrast to the quantitative measure of chronological age, biological aging is more qualitative and can be influenced by factors including genetic background, lifestyle, nutrition, socio-economic status, and environmental influences such as access to health care $(2,3)$. The biological processes involved in aging are complex and are predominantly driven by damage acquired by cells and tissues over time (4), thereby defining aging as the progressive decline of function through the loss of physiological integrity (5).

Frailty is a late-life syndrome resulting from cumulative decline across various physiological reserves. An older adult with frailty has an increased vulnerability to stressors and consequently develops adverse health-related outcomes such as disability, increased dependency, comorbidities, and mortality (6-8). Deterioration of the musculoskeletal system is the main component of frailty, but also other physical domains, including cognitive, psychological, and social domains, can be affected (8). Physical frailty often involves sarcopenia, which is defined as decreased muscle mass and function, including muscle strength and physical performance (9). Sarcopenia has implications on mobility, the risk of falls, and the onset of insulin resistance, type 2 diabetes and obesity $(5,10)$. In turn, the development of frailty and sarcopenia can be accelerated under the influence of metabolic disturbances, nutritional deficiencies, lack of physical activity, and comorbidities (9), together leading to a vicious cycle. Skeletal muscle mass starts to decline with age from around the age of 40 years (11), and even more prominent is the age-related decline in muscle strength, which is two to five times faster than the decline in muscle mass and is a more consistent risk for disability (12). To better understand the aging process, the present thesis aimed to investigate the age-related changes in the skeletal muscle and its consequences on physical performance and metabolic health. In chapter 3, irrespective of lean mass, muscle strength was remarkably lower in older adults than in young individuals. The age-related decline in muscle mass seems to be attributable to muscle fiber atrophy and muscle fiber loss of especially type II fibers, contributing to muscle weakness (13-15). Nevertheless, despite changes in muscle strength, we did not observe age-related differences in 
muscle fiber characteristics between young and older participants who, important to mention, had comparable levels of physical activity (chapter 3). The discrepancy could be explained by the fact that previous studies did not measure and control for an age-related decrease in physical activity (13-15), which could explain muscle fiber atrophy (16). These results suggest that muscle fiber atrophy is not simply a function of aging per se but might also be driven by age-related physical inactivity and immobilization.

One of the most critical consequences of age-related disability is the increased risk of falls leading to further functional decline and physical impairments (17). As such, numerous exercise training programs in older adults aim to prevent the loss of gait stability and improve physical independence. It is commonly assumed that muscle strength and power are essential factors for coping with large balance disturbances. Nevertheless, muscle strength training programs usually only moderately improve balance and fall management (18-20). In addition, in chapter 6, no correlations were observed between muscle fiber characteristics, muscle volume and strength, and stability recovery performance in young and older participants with varying physical activity levels. These results suggest that muscle tissue properties per se are not key factors for balance recovery and a multi-domain intervention might be demanded to mitigate physical aging.

\section{Mitochondrial dysfunction and aging}

Because mitochondria are essential regulators of processes such as ATP provision, oxidative stress, proteostasis, apoptosis, inflammation, and $\mathrm{Ca}^{2+}$ handling (21-24), impairments in mitochondrial function potentially impact the progression of sarcopenia and accelerate aging. Indeed, mitochondrial function is widely studied in relation to aging, as exemplified by numerous studies covering various aspects of mitochondrial capacity and function in human skeletal muscle.

First of all, studies have focused on the effect of age on (markers of) mitochondrial density. Thus, using citrate synthase (CS) activity as a marker of cellular oxidative capacity and mitochondrial density (25), several studies reported reductions in mitochondrial density with age $(26,27)$. In contrast to these findings, we did not show a significant difference in mitochondrial density in older adults compared to young 
individuals, based on OXPHOS protein levels (chapter 3). Also, other studies reported no age-related differences in mitochondrial content based on CS activity and OXPHOS protein expression $(28,29)$. However, despite a comparable mitochondrial density, we observed that mitochondrial respiratory capacity was lower in older adults than young individuals (chapter 3), supporting the idea that mitochondrial function can change with age in the absence of perceptible changes in mitochondrial volume (30). Although the observed age-related decline in mitochondrial respiratory capacity in older adults is in line with previous clinical work (31-33), other studies observed no chronological age effect on mitochondrial respiration $(28,34,35)$. Factors like age-related changes in physical activity may explain these differences between studies.

Besides mitochondrial content and (intrinsic) respiratory capacity, recent studies (36-40) have highlighted the importance of mitochondrial morphology and turnover for proper mitochondrial function (reviewed in chapter 2). Mitochondrial integrity relies on the efficiency of quality control processes, and their mitochondrial network is regulated by continuous fusion, fission, and mitophagy (41-43). While the activation of the mitochondrial biogenesis pathway leads to an increase in mitochondrial content, e.g. as a result of exercise training, it is also vital to eliminate any mitochondrial segments that have outlived their usefulness via mitophagy in order to maintain or improve the quality of the mitochondrial pool (44-46). Limited animal and human studies have examined mitochondrial quality control processes in muscle aging, with inconsistent results, and therefore warrant further investigation. Thus, some studies described increased fission in muscle tissue of older rats, indicated by smaller mitochondria and increased expression of fission proteins FIS1 and DRP1 (47), whereas other studies indicated an increased fusion, illustrated by a larger mitochondrial reticulum size, along with concomitant increases in the fusion proteins OPA1 and MFN2 increased fusion in aged mice (48). Furthermore, other studies reported no differences in mRNA levels for mitochondrial fission protein Fis1 and fusion protein Mitofusin 1 (Mfn1) between young and old sedentary individuals $(49,50)$. The muscle tissue available from the young and older adults studied in chapter 3 may help to understand age-related changes through assessment of fission, fusion, and mitophagy proteins and their relationship with muscle health and physical function. Moreover, combining the measurement of these regulatory 
proteins with confocal microscopy could shed further light on age-related alterations in the regulation of the finely tuned mitochondrial network. Nonetheless, it should be stressed that these experiments would only provide a snapshot of mitochondrial connectivity, whereas we know from previous studies that the network is highly dynamic and already displays changes over the course of a day (51)

\section{Potential confounders of the relationship between aging and mitochondrial capacity}

Some of the contradictory associations between aging and skeletal muscle mitochondrial capacity and integrity, particularly in humans, could relate to heterogeneous participant characteristics such as physical activity, body fat content/distribution, and insulin sensitivity $(28,52)$. In other words, the isolated impact of aging on skeletal muscle mitochondrial function is difficult to unravel from the many other factors that change concurrently with aging (53).

Aging has been associated with an increased abdominal white adipose tissue mass $(54,55)$. In chapter 3 we also observed that body fat content was different between young and older adults and may therefore contribute to the reduction in mitochondrial function and physical capacity. Aging-related adiposity is also linked with increased fat deposition in skeletal muscle (56). Thus, in the $m$. vastus lateralis of older adults, intramyocellular lipid (IMCL) accumulation has been observed by proton magnetic resonance spectroscopy ( $\left.{ }^{1} \mathrm{H}-\mathrm{MRS}\right)(57)$ as well as by histological analysis of muscle biopsies (58,59). Moreover, larger IMCL droplets have been linked to decreased mitochondrial content in muscle fibers from older adults, and lipid droplet morphology likely contributes to age-related reductions in mitochondrial function and lipid metabolism (52). Also, reduction in peroxisome proliferatoractivated receptor gamma coactivator $1 \alpha$ (PGC-1 $\alpha$ ) expression and disruption of mitochondrial dynamics and autophagy have been linked to lipid accumulation in muscle fibers during aging (60). Nevertheless, it remains to be established to which extent IMCL per se mediates the decline in mitochondrial function and if changes in mitochondrial capacity are a cause or consequence of increased adiposity.

In addition to changes in body composition, aging is generally associated with decreased physical activity levels, and previous studies suggested that mitochondrial function is not affected by chronological aging per se but rather by the age-related 
decrease in physical activity $(34,61,62)$. Sedentary, older individuals exhibit impaired mitochondrial function, possible loss of membrane potential, and increased oxidative stress $(63,64)$. These deleterious changes promote the loss of muscle endurance and strength associated with age and impair life quality (64-66). Interestingly though, our findings described in chapter 3 indicate that even when maintaining an adequate daily physical activity level, reflected by $\quad 10000$ steps per day, a decline in mitochondrial function and muscle health at older age is inevitable. However, it should be stressed that this does not exclude moderate physical activity as an important factor in preventing more severe deterioration of skeletal muscle mitochondrial capacity and muscle function at older age. Older adults with low(er) physical activity levels and impaired physical function were underrepresented in our cohort and, likely, this group still benefits from increases in moderate physical activity. Indeed, in larger cohorts of older adults, the number of steps per day correlated with the prevention of falls (67) and all-cause mortality (68). Nonetheless, only participants with an even higher physical activity level, achieved through regular physical exercise training, were able to negate the effects of aging on skeletal muscle mitochondrial capacity and muscle function in our study (chapter 3 ). In line with these results, modest aerobic activity, reported as $\sim 3.5 \mathrm{~h} /$ week of leisure-time activity, fails to prevent the age-related decrease in mitochondrial respiration and insulin sensitivity. Simultaneously, rigorous exercise programs such as high-intensity interval training can normalize mitochondrial respiration in older adults $(69,70)$, underscoring the importance of intensity of exercise as a determinant of ensuring healthy aging.

\section{Can physical exercise training prevent biological aging?}

While physical activity is defined as any bodily movement produced by skeletal muscles resulting in energy expenditure, physical exercise specifically refers to planned, structured, repetitive, and purposive activity to improve or maintain physical fitness (71). Given its whole-body, health-promoting nature, physical exercise is one of the most powerful tools to prevent functional decline and the onset of numerous age-related chronic diseases (72,73). As regular exercise training promotes independence in older adults, it is a key component of successful aging (74). 
Moreover, the 2018 Physical Activity Guidelines emphasize the importance of regular exercise as a medical therapy in older individuals (75). Benefits of exercise training in older adults have been demonstrated in multiple diseases and disorders, including cancer, type 2 diabetes, peripheral artery disease, hypertension, osteoporosis, dyslipidemia, obesity, cognitive impairment, depression, anxiety, and sleep disorders (75). An important goal of exercise interventions in older adults is to preserve skeletal muscle function to prevent physical disability (76). For example, resistance training in aged individuals increases muscle mass and strength, preventing sarcopenia and age-related muscle weakness (77). Furthermore, endurance training improves aerobic capacity and physical function, supporting independence in daily living activities (78). Moreover, exercise can also prevent ageassociated intermuscular adipose tissue infiltration and the associated development of insulin resistance and poor muscle function (79).

As mentioned above, we found (chapter 3 ) that older adults who regularly perform exercise training display an improved skeletal muscle mitochondrial capacity and muscle function compared to non-exercising older individuals. This was accompanied by an increased insulin sensitivity and more favorable body composition, with a lower percentage of overweight observed amongst the trained older adults compared to the normally active and inactive older adults included in this study. Physical exercise is well known to increase mitochondrial content and function, largely through stimulation of PGC-1 $1 \alpha$ activity $(80,81)$. Furthermore, exercise can improve the efficiency of mitochondria through remodeling of the mitochondrial network resulting from an improved balance between mitochondrial biogenesis, fusion, fission and mitophagy (82-85). Moreover, in response to repeated bouts of endurance exercise, several studies have presented increases in autophagy and mitophagy markers within the muscle (45), essential in maintaining a healthy mitochondrial pool.

Despite the extensively recognized benefits of exercise training in maintaining physical function during aging, only $23.5 \%$ of the aging population succeeds in meeting the WHO recommended physical exercise levels of at least 150 minutes of moderate-intensity aerobic physical exercise per week (86). Furthermore, highly structured exercise regimes may not be realistic for all older individuals, especially when they are affected by comorbidities and have a poor physical function. Even 
when older adults engage in carefully controlled exercise training regimens, the training response is heterogeneous $(87,88)$. In older exercise responders, a large interindividual variability was observed in the improvement of functional performance assessed by either maximal oxygen uptake (VO2max) and walking speed after endurance training or by knee extensor strength after resistance training (88). Moreover, a fraction of older participants does not respond or even declines in function following exercise training and shows apparent adverse effects on cardiometabolic parameters such as resting systolic blood pressure, triglycerides, and insulin levels $(87,88)$. Although specific mechanisms have been proposed to explain the decreased response to exercise in older adults, including the age-related decline in the bone-derived hormone osteocalcin (89), there are likely multiple factors at play, which deserve more investigation.

\section{Exercise responsiveness and NAD ${ }^{+}$}

The variability in exercise training responsiveness might relate to the downregulation of nicotinamide adenine dinucleotide $\left(\mathrm{NAD}^{+}\right)$and the $\mathrm{NAD}^{+}$metabolome at older age. $\mathrm{NAD}^{+}$is a pyridine nucleotide that serves as an essential cofactor and substrate for many critical cellular processes involved in DNA repair, oxidative phosphorylation and ATP production, epigenetically modulated gene expression, immunological functions and intracellular calcium signaling (90). NAD ${ }^{+}$content in skeletal muscle in the resting state is positively correlated with the oxidative capacity of skeletal muscle fibers (90). Exercise requires a higher $\mathrm{NAD}^{+}$recycling rate to cope with the increased energetic demand, and hence changes in $\mathrm{NAD}^{+}$levels may provide a logical connection between exercise-induced metabolic stress and muscle adaptation (91). More specifically, exercise induces local energy deprivation and increases NAD ${ }^{+}$ levels, activating the $\mathrm{NAD}^{+}$-dependent deacetylase SIRT1 and other sirtuins that control the physiological responses to exercise. SIRT1, in turn, activates PGC-1a to induce mitochondrial biogenesis and fatty acid oxidation (92). Decreased NAD ${ }^{+}$ levels in aged mice have been linked to reduced activity of SIRT1 and mitochondrial activity (93-95), suggesting that inadequate exercise responses could be related to the observed decline in $\mathrm{NAD}^{+}$upon aging. 
Both underproduction and overconsumption of $\mathrm{NAD}^{+}$may contribute to $\mathrm{NAD}^{+}$ depletion in aging. The rate-limiting enzyme in $\mathrm{NAD}^{+}$biosynthesis is nicotinamide phosphoribosyl transferase (NAMPT) $(96,97)$ and NAMPT was shown to be negatively correlated with age in human skeletal muscle, indicating a possible agerelated decline in $\mathrm{NAD}^{+}$synthesis (98). $\mathrm{NAD}^{+}$overconsumption results from an overactive response to DNA damage by poly (ADP ribose) polymerases (PARPs) and an age-related increase in CD38, as shown in murine and human muscle tissue $(95,96,99)$. Numerous animal studies have indicated NAD ${ }^{+}$depletion as the primary cause of disease and disability during aging (100). Nevertheless, very few studies have established that $\mathrm{NAD}^{+}$also declines with aging in human tissues (101). In chapter 4, we therefore performed a metabolomic analysis in skeletal muscle biopsies. We identified $\mathrm{NAD}^{+}$as one of the top 3 metabolites decreasing with age in skeletal muscle tissue in older adults compared to young adults with similar and adequate physical activity levels. Moreover, $\mathrm{NAD}^{+}$was more severely depleted in physically impaired older adults, whereas exercise-trained older adults were nearly fully protected against this age-related loss of $\mathrm{NAD}^{+}$. Interestingly, we also found strong correlations between muscle $\mathrm{NAD}^{+}$content and the subjects' daily step count, mitochondrial capacity, and muscle function. Our results affirm the relation of NAD ${ }^{+}$ with muscle health in aging human individuals and highlight the NAD+-pathway as a promising target to promote healthy aging, possibly in combination with exercise training.

Indeed, based on a large number of preclinical studies in invertebrate lab models and mice, $\mathrm{NAD}^{+}$-based therapeutics have been proposed as one of the most promising strategies to counteract physiological aging (96,100-102). More specifically, supplementation of the $\mathrm{NAD}^{+}$precursor nicotinamide mononucleotide $(\mathrm{NMN})$ in aging mice attenuated age-related decline of muscle strength, mitigated the age-associated decline in energy metabolism, insulin sensitivity and lipid metabolism, and increased lifespan and healthspan (102). In addition, treatment with the $\mathrm{NAD}^{+}$precursor nicotinamide riboside (NR) in aged mice prevented muscle stem cell senescence, improved mitochondrial function and increased lifespan (103). Another strategy to increase $\mathrm{NAD}^{+}$bioavailability is by inhibiting the $\mathrm{NAD}^{+}$ consuming enzymes CD38 en PPAR, reducing enzymatic competition, and enhancing SIRT1 activity. Long-term inhibition of the NAD+ consuming enzyme 
PARP in mice enhanced SIRT1 activity, which was accompanied by improved mitochondrial function and enhanced energy expenditure and endurance performance (104). Nevertheless, evidence on the efficacy of NAD ${ }^{+}$supplementation in improving physical function in humans is limited. NAD ${ }^{+}$can be derived from various dietary sources as it can be synthesized (1) de novo from the essential amino acid L-tryptophan, (2) through the Preiss-Handler pathway from vitamin B3 compounds such as nicotinic acid (NA, niacin), or (3) through the salvage pathway from nicotinamide, NMN, and NR (105). Chapter 5 investigated the efficacy of $\mathrm{NAD}^{+}$-precursor supplementation with L-tryptophan, nicotinic acid, and nicotinamide in older adults with an impaired physical function. However, NAD ${ }^{+}$precursor supplementation did neither affect skeletal muscle mitochondrial respiratory capacity nor physical function. These findings are in line with data from Elhassan et al. (106), who supplemented healthy, older adults with a high dose of NR and also did not observe any alterations in mitochondrial respiration capacity and muscle strength. In fact, several other studies, performed in obese individuals $(107,108)$, middle-aged participants (109), and older men (110), failed to establish beneficial effects of NR supplementation on mitochondrial capacity and metabolic health.

Based on these mixed findings in animals vs. humans, NAD ${ }^{+}$precursor supplementation alone might not be sufficient to improve in physical performance. Considering that the positive effects of regular exercise training may be limited in older adults by the dysregulated $\mathrm{NAD}^{+}$metabolome, it is conceivable that the combination of exercise training with $\mathrm{NAD}^{+}$precursor supplementation could potentiate exercise therapy in older adults. Preclinical studies on the combination of NR $(48,49)$ or NMN (113) with exercise training have reported mixed results. These studies were conducted mainly on young animals and used different exercise protocols. Nevertheless, NR has a good safety profile and showed beneficial effects in individuals with impaired baseline health status, as shown in patients with myopathies (114). Additional well-designed preclinical studies and RCTs in humans are warranted to test the combination of $\mathrm{NAD}^{+}$precursor supplementation and exercise in improving the homeostasis of the $\mathrm{NAD}^{+}$metabolome, mitochondrial function and exercise tolerance in older adults with moderate physical function.

Taken together, physical exercise training is a powerful tool to prevent the decline in physical integrity with age but might not be feasible and equally effective for all older 
individuals. Nutritional supplementation of $\mathrm{NAD}^{+}$precursors might facilitate and optimize the adaptive process to exercise training in older adults, and as such, may boost the efficacy of exercise therapy for healthy aging.

\section{Concluding remarks}

This thesis aimed to study skeletal muscle mitochondria's role in the age-related loss of physical function. Based on the results, we conclude that, despite maintaining recommended daily physical activity levels, aging is associated with a decline in mitochondrial capacity, NAD+ levels, muscle health, and physical function. Higher levels of physical activity, achieved through high-intensity regular exercise training, partially prevent the effects of aging on $\mathrm{NAD}^{+}$abundance, mitochondrial function, and muscle health. The observed correlations between mitochondrial capacity and exercise efficiency and insulin sensitivity highlight the role of mitochondrial dysfunction in accelerated aging and deterioration of skeletal muscle health. Moreover, $\mathrm{NAD}^{+}$metabolism strongly correlates with skeletal muscle health during aging as assessed through mitochondrial and physical functioning, affirming the relation of $\mathrm{NAD}^{+}$with overall health status, especially in older adults, and emphasizing the NAD-pathway as a promising target to promote healthy aging in humans. Nevertheless, $\mathrm{NAD}^{+}$supplementation with $\mathrm{NAD}^{+}$-precursors alone did not improve mitochondrial function and muscle health. Moreover, we conclude that despite differences in fiber type distribution across the different age groups, muscle tissue properties per se are not key factors for balance recovery, highlighting that fall prevention training should include the multiple aspects of physical decline with aging.

Taken together, mitochondria represent a significant target in the strategy to extend healthspan. To this point, exercise remains the most effective behavioral medicine for improving mitochondrial health, not only in muscle but also in other tissues. Further elucidation of mitochondrial turnover mechanisms and NAD ${ }^{+}$metabolism in muscle could offer potential therapeutic targets to promote healthy aging in humans. 


\section{REFERENCES}

1. Levine, M. E. Modeling the Rate of Senescence: Can Estimated Biological Age Predict Mortality More Accurately Than Chronological Age? The Journals of Gerontology Series A: Biological Sciences and Medical Sciences 68, 667-674 (2013).

2. Blackburn, E. H., Epel, E. S. \& Lin, J. Human telomere biology: A contributory and interactive factor in aging, disease risks, and protection. Science 350, 1193-1198 (2015).

3. Ridout, K. K. et al. Early life adversity and telomere length: a meta-analysis. Mol Psychiatry 23, 858-871 (2018).

4. Akbari, M., Kirkwood, T. B. L. \& Bohr, V. A. Mitochondria in the signaling pathways that control longevity and health span. Ageing Research Reviews 54, 100940 (2019).

5. López-Otín, C., Blasco, M. A., Partridge, L., Serrano, M. \& Kroemer, G. The Hallmarks of Aging. Cell 153, 1194-1217 (2013).

6. Morley, J. E. et al. Frailty Consensus: A Call to Action. J Am Med Dir Assoc 14, 392397 (2013).

7. Walston, J. et al. Research agenda for frailty in older adults: toward a better understanding of physiology and etiology: summary from the American Geriatrics Society/National Institute on Aging Research Conference on Frailty in Older Adults. $J$ Am Geriatr Soc 54, 991-1001 (2006).

8. Gielen, E. et al. Musculoskeletal Frailty: A Geriatric Syndrome at the Core of Fracture Occurrence in Older Age. Calcif Tissue Int 91, 161-177 (2012).

9. Angulo, J., El Assar, M. \& Rodríguez-Mañas, L. Frailty and sarcopenia as the basis for the phenotypic manifestation of chronic diseases in older adults. Mol Aspects Med 50, 1-32 (2016).

10. Carter, H. N., Chen, C. C. W. \& Hood, D. A. Mitochondria, muscle health, and exercise with advancing age. Physiology (Bethesda) 30, 208-223 (2015).

11. Silva, A. M. et al. Ethnicity-related skeletal muscle differences across the lifespan. Am J Hum Biol 22, 76-82 (2010).

12. Mitchell, W. K. et al. Sarcopenia, Dynapenia, and the Impact of Advancing Age on Human Skeletal Muscle Size and Strength; a Quantitative Review. Front. Physio. 3, (2012).

13. Kramer, I. F. et al. Extensive Type II Muscle Fiber Atrophy in Elderly Female Hip Fracture Patients. The Journals of Gerontology: Series A 72, 1369-1375 (2017).

14. McPhee, J. S. et al. The Contributions of Fiber Atrophy, Fiber Loss, In Situ Specific Force, and Voluntary Activation to Weakness in Sarcopenia. The Journals of Gerontology: Series A 73, 1287-1294 (2018).

15. Nilwik, R. et al. The decline in skeletal muscle mass with aging is mainly attributed to a reduction in type II muscle fiber size. Exp Gerontol 48, 492-498 (2013). 
16. Mosole, S. et al. Long-term high-level exercise promotes muscle reinnervation with age. J Neuropathol Exp Neurol 73, 284-294 (2014).

17. Terroso, M., Rosa, N., Torres Marques, A. \& Simoes, R. Physical consequences of falls in the elderly: a literature review from 1995 to 2010. Eur Rev Aging Phys Act 11, 51-59 (2014).

18. Orr, R., Raymond, J. \& Fiatarone Singh, M. Efficacy of Progressive Resistance Training on Balance Performance in Older Adults: A Systematic Review of Randomized Controlled Trials. Sports Medicine 38, 317-343 (2008).

19. Horlings, C. G. C., van Engelen, B. G. M., Allum, J. H. J. \& Bloem, B. R. A weak balance: the contribution of muscle weakness to postural instability and falls. Nat Clin Pract Neurol 4, 504-515 (2008).

20. Sherrington, C. et al. Exercise for preventing falls in older people living in the community. Cochrane Database Syst Rev 1, CD012424 (2019).

21. Heber, D. et al. Clinical detection of sarcopenic obesity by bioelectrical impedance analysis. The American Journal of Clinical Nutrition 64, 472S-477S (1996).

22. Rapizzi, E. et al. Recombinant expression of the voltage-dependent anion channel enhances the transfer of Ca2+ microdomains to mitochondria. J Cell Biol 159, 613624 (2002).

23. Kujoth, G. C. et al. Mitochondrial DNA mutations, oxidative stress, and apoptosis in mammalian aging. Science 309, 481-484 (2005).

24. Schaap, L. A., Pluijm, S. M. F., Deeg, D. J. H. \& Visser, M. Inflammatory markers and loss of muscle mass (sarcopenia) and strength. Am J Med 119, 526.e9-17 (2006).

25. Larsen, S. et al. The influence of age and aerobic fitness: effects on mitochondrial respiration in skeletal muscle. Acta Physiol (Oxf) 205, 423-432 (2012).

26. Short, K. R. et al. Decline in skeletal muscle mitochondrial function with aging in humans. Proceedings of the National Academy of Sciences 102, 5618-5623 (2005).

27. Short, K. R. et al. Impact of aerobic exercise training on age-related changes in insulin sensitivity and muscle oxidative capacity. Diabetes 52, 1888-1896 (2003).

28. Rasmussen, U. F., Krustrup, P., Kjaer, M. \& Rasmussen, H. N. Experimental evidence against the mitochondrial theory of aging. A study of isolated human skeletal muscle mitochondria. Exp Gerontol 38, 877-886 (2003).

29. Gouspillou, G. et al. Increased sensitivity to mitochondrial permeability transition and myonuclear translocation of endonuclease $\mathrm{G}$ in atrophied muscle of physically active older humans. FASEB j. 28, 1621-1633 (2014).

30. Hood, D. A., Memme, J. M., Oliveira, A. N. \& Triolo, M. Maintenance of Skeletal Muscle Mitochondria in Health, Exercise, and Aging. Annu. Rev. Physiol. 81, 19-41 (2019). 
31. Tonkonogi, M. et al. Reduced oxidative power but unchanged antioxidative capacity in skeletal muscle from aged humans. Pflugers Arch - Eur J Physiol 446, 261-269 (2003).

32. Trounce, I., Byrne, E. \& Marzuki, S. Decline in skeletal muscle mitochondrial respirationy chain function - possible factor in aging. The Lancet (1989).

33. Boffoli, D. et al. Decline with age of the respiratory chain activity in human skeletal muscle. Biochimica et Biophysica Acta (BBA) - Molecular Basis of Disease 1226, 7382 (1994).

34. Distefano, G. et al. Physical activity unveils the relationship between mitochondrial energetics, muscle quality, and physical function in older adults: Mitochondria, muscle quality and physical function in aging. Journal of Cachexia, Sarcopenia and Muscle 9, 279-294 (2018).

35. Distefano, G. et al. Chronological Age Does not Influence Ex-vivo Mitochondrial Respiration and Quality Control in Skeletal Muscle. GERONA glw102 (2016). doi:10.1093/gerona/glw102

36. Liu, H. et al. Aberrant mitochondrial morphology and function associated with impaired mitophagy and DNM1L-MAPK/ERK signaling are found in aged mutant Parkinsonian LRRK2R1441G mice. Autophagy 1-25 (2020). doi:10.1080/15548627.2020.1850008

37. Liu, Y. J. et al. Mitochondrial translation and dynamics synergistically extend lifespan in C. elegans through HLH-30. J Cell Biol 219, (2020).

38. Yang, X. et al. Mitochondrial dynamics quantitatively revealed by STED nanoscopy with an enhanced squaraine variant probe. Nat Commun 11, 3699 (2020).

39. Liu, Y. J., Mclntyre, R. L., Janssens, G. E. \& Houtkooper, R. H. Mitochondrial fission and fusion: A dynamic role in aging and potential target for age-related disease. Mechanisms of Ageing and Development 186, 111212 (2020).

40. Romanello, V. \& Sandri, M. The connection between the dynamic remodeling of the mitochondrial network and the regulation of muscle mass. Cell. Mol. Life Sci. (2020). doi:10.1007/s00018-020-03662-0

41. Ono, T., Isobe, K., Nakada, K. \& Hayashi, J.-I. Human cells are protected from mitochondrial dysfunction by complementation of DNA products in fused mitochondria. Nature Genetics 28, 272-275 (2001).

42. Twig, G. et al. Fission and selective fusion govern mitochondrial segregation and elimination by autophagy. EMBO J 27, 433-446 (2008).

43. Twig, G., Hyde, B. \& Shirihai, O. S. Mitochondrial fusion, fission and autophagy as a quality control axis: The bioenergetic view. Biochimica et Biophysica Acta (BBA) Bioenergetics 1777, 1092-1097 (2008).

44. Ljubicic, V. et al. Molecular basis for an attenuated mitochondrial adaptive plasticity in aged skeletal muscle. Aging (Albany NY) 1, 818-830 (2009). 
45. Lira, V. A. et al. Autophagy is required for exercise training-induced skeletal muscle adaptation and improvement of physical performance. FASEB J 27, 4184-4193 (2013).

46. Kim, Y., Triolo, M., Erlich, A. T. \& Hood, D. A. Regulation of autophagic and mitophagic flux during chronic contractile activity-induced muscle adaptations. Pflugers Arch 471, 431-440 (2019).

47. Iqbal, S., Ostojic, O., Singh, K., Joseph, A.-M. \& Hood, D. A. Expression of mitochondrial fission and fusion regulatory proteins in skeletal muscle during chronic use and disuse. Muscle Nerve 48, 963-970 (2013).

48. Leduc-Gaudet, J.-P. et al. Mitochondrial morphology is altered in atrophied skeletal muscle of aged mice. Oncotarget 6, 17923-17937 (2015).

49. Bori, Z. et al. The effects of aging, physical training, and a single bout of exercise on mitochondrial protein expression in human skeletal muscle. Exp Gerontol 47, 417424 (2012).

50. Konopka, A. R., Suer, M. K., Wolff, C. A. \& Harber, M. P. Markers of human skeletal muscle mitochondrial biogenesis and quality control: effects of age and aerobic exercise training. J Gerontol A Biol Sci Med Sci 69, 371-378 (2014).

51. Daemen, S. C.M. A microscopic view on myocellular lipid droplets and mitochondria: Dynamic organelles involved in insulin sensitivity. (2019).

52. Crane, J. D., Devries, M. C., Safdar, A., Hamadeh, M. J. \& Tarnopolsky, M. A. The effect of aging on human skeletal muscle mitochondrial and intramyocellular lipid ultrastructure. J Gerontol A Biol Sci Med Sci 65, 119-128 (2010).

53. Distefano, G. \& Goodpaster, B. H. Effects of Exercise and Aging on Skeletal Muscle. Cold Spring Harb Perspect Med 8, a029785 (2018).

54. Miard, S. \& Picard, F. Obesity and aging have divergent genomic fingerprints. Int J Obes (Lond) 32, 1873-1874 (2008).

55. Barzilai, N., Huffman, D. M., Muzumdar, R. H. \& Bartke, A. The critical role of metabolic pathways in aging. Diabetes 61, 1315-1322 (2012).

56. Slawik, M. \& Vidal-Puig, A. J. Lipotoxicity, overnutrition and energy metabolism in aging. Ageing Res Rev 5, 144-164 (2006).

57. Cree, M. G. et al. Intramuscular and Liver Triglycerides Are Increased in the Elderly. The Journal of Clinical Endocrinology \& Metabolism 89, 3864-3871 (2004).

58. St-Jean-Pelletier, F. et al. The impact of ageing, physical activity, and pre-frailty on skeletal muscle phenotype, mitochondrial content, and intramyocellular lipids in men: Muscle phenotype, mitochondrial mass, and lipid content in human ageing. Journal of Cachexia, Sarcopenia and Muscle 8, 213-228 (2017).

59. Gueugneau, M. et al. Skeletal muscle lipid content and oxidative activity in relation to muscle fiber type in aging and metabolic syndrome. J Gerontol A Biol Sci Med Sci 70, 566-576 (2015). 
60. Zhao, L. et al. Evidence for association of mitochondrial metabolism alteration with lipid accumulation in aging rats. Experimental Gerontology 56, 3-12 (2014).

61. Barrientos, A. et al. Absence of relationship between the level of electron transport chain activities and aging in human skeletal muscle. Biochem Biophys Res Commun 229, 536-539 (1996).

62. Brierley, E. J., Johnson, M. A., James, O. F. \& Turnbull, D. M. Effects of physical activity and age on mitochondrial function. QJM 89, 251-258 (1996).

63. Chabi, B. et al. Mitochondrial function and apoptotic susceptibility in aging skeletal muscle. Aging Cell 7, 2-12 (2008).

64. Montgomery, M. K. \& Turner, N. Mitochondrial dysfunction and insulin resistance: an update. Endocrine Connections 4, R1-R15 (2015).

65. Payne, B. A. I. \& Chinnery, P. F. Mitochondrial dysfunction in aging: Much progress but many unresolved questions. Biochim Biophys Acta 1847, 1347-1353 (2015).

66. Romanello, V. \& Sandri, M. Mitochondrial Quality Control and Muscle Mass Maintenance. Front Physiol 6, 422 (2015).

67. Aranyavalai, T. et al. Association between walking 5000 step/day and fall incidence over six months in urban community-dwelling older people. BMC Geriatr 20, 194 (2020).

68. Lee, I.-M. et al. Association of Step Volume and Intensity With All-Cause Mortality in Older Women. JAMA Intern Med (2019). doi:10.1001/jamainternmed.2019.0899

69. Lanza, I. R. et al. Endurance Exercise as a Countermeasure for Aging. Diabetes 57, 2933-2942 (2008).

70. Robinson, M. M. et al. Enhanced Protein Translation Underlies Improved Metabolic and Physical Adaptations to Different Exercise Training Modes in Young and Old Humans. Cell Metabolism 25, 581-592 (2017).

71. Caspersen, C. J., Powell, K. E. \& Christenson, G. M. Physical activity, exercise, and physical fitness: definitions and distinctions for health-related research. Public Health Rep 100, 126-131 (1985).

72. Booth, F. W. Lack of exercise is a major cause of chronic diseases. 135 (2014).

73. Ruegsegger, G. N. \& Booth, F. W. Health Benefits of Exercise. 15

74. Anton, S. D. et al. Successful aging: Advancing the science of physical independence in older adults. Ageing Research Reviews 24, 304-327 (2015).

75. Piercy, K. L. et al. The Physical Activity Guidelines for Americans. JAMA 320, 2020 (2018).

76. Pahor, M. et al. Effect of Structured Physical Activity on Prevention of Major Mobility Disability in Older Adults: The LIFE Study Randomized Clinical Trial. JAMA 311, 2387 (2014). 
77. Borde, R., Hortobágyi, T. \& Granacher, U. Dose-Response Relationships of Resistance Training in Healthy Old Adults: A Systematic Review and Meta-Analysis. Sports Med 45, 1693-1720 (2015).

78. Bouaziz, W. et al. Health benefits of aerobic training programs in adults aged 70 and over: a systematic review. Archives of Gerontology and Geriatrics 69, 110-127 (2017).

79. Goodpaster, B. H. et al. Effects of physical activity on strength and skeletal muscle fat infiltration in older adults: a randomized controlled trial. J Appl Physiol 105, 6 (2008).

80. Baar, K. et al. Adaptations of skeletal muscle to exercise: rapid increase in the transcriptional coactivator PGC-1. FASEB j. 16, 1879-1886 (2002).

81. Geng, T. et al. PGC-1a plays a functional role in exercise-induced mitochondrial biogenesis and angiogenesis but not fiber-type transformation in mouse skeletal muscle. American Journal of Physiology-Cell Physiology 298, C572-C579 (2010).

82. Cartoni, R. et al. Mitofusins $1 / 2$ and ERRalpha expression are increased in human skeletal muscle after physical exercise. J Physiol 567, 349-358 (2005).

83. Ding, $\mathrm{H}$. et al. Response of mitochondrial fusion and fission protein gene expression to exercise in rat skeletal muscle. Biochim Biophys Acta 1800, 250-256 (2010).

84. Perry, C. G. R. et al. Repeated transient mRNA bursts precede increases in transcriptional and mitochondrial proteins during training in human skeletal muscle. $J$ Physiol 588, 4795-4810 (2010).

85. Smuder, A. J., Kavazis, A. N., Min, K. \& Powers, S. K. Exercise protects against doxorubicin-induced markers of autophagy signaling in skeletal muscle. $J \mathrm{Appl}$ Physiol (1985) 111, 1190-1198 (2011).

86. Koyanagi, A., Stubbs, B., Smith, L., Gardner, B. \& Vancampfort, D. Correlates of physical activity among community-dwelling adults aged 50 or over in six low- and middle-income countries. PLoS ONE 12, e0186992 (2017).

87. Bouchard, C. et al. Adverse Metabolic Response to Regular Exercise: Is It a Rare or Common Occurrence? PLoS ONE 7, e37887 (2012).

88. Chmelo, E. A. et al. Heterogeneity of Physical Function Responses to Exercise Training in Older Adults. J Am Geriatr Soc 63, 462-469 (2015).

89. Mera, P. et al. Osteocalcin signaling in myofibers is necessary and sufficient for optimum adaptation to exercise. Cell Metab 23, 1078-1092 (2016).

90. Graham, T., Sjøgaard, G., Löllgen, H. \& Saltin, B. NAD in muscle of man at rest and during exercise. Pflugers Arch 376, 35-39 (1978).

91. White, A. T. \& Schenk, S. NAD + /NADH and skeletal muscle mitochondrial adaptations to exercise. American Journal of Physiology-Endocrinology and Metabolism 303, E308-E321 (2012). 
92. Haigis, M. C. \& Sinclair, D. A. Mammalian sirtuins: biological insights and disease relevance. Annu Rev Pathol 5, 253-295 (2010).

93. Gomes, A. P. et al. Declining NAD+ Induces a Pseudohypoxic State Disrupting Nuclear-Mitochondrial Communication during Aging. Cell 155, 1624-1638 (2013).

94. Braidy, N. et al. Age Related Changes in NAD+ Metabolism Oxidative Stress and Sirt1 Activity in Wistar Rats. PLoS ONE 6, 18 (2011).

95. Camacho-Pereira, J. et al. CD38 dictates age-related NAD decline and mitochondrial dysfunction through a SIRT3-dependent mechanism. Cell Metab 23, 1127-1139 (2016).

96. Braidy, N. et al. Role of Nicotinamide Adenine Dinucleotide and Related Precursors as Therapeutic Targets for Age-Related Degenerative Diseases: Rationale, Biochemistry, Pharmacokinetics, and Outcomes. Antioxid Redox Signal 30, 251-294 (2019).

97. Revollo, J. R., Grimm, A. A. \& Imai, S. The NAD Biosynthesis Pathway Mediated by Nicotinamide Phosphoribosyltransferase Regulates Sir2 Activity in Mammalian Cells. J. Biol. Chem. 279, 50754-50763 (2004).

98. de Guia, R. M. et al. Aerobic and resistance exercise training reverses agedependent decline in NAD+ salvage capacity in human skeletal muscle. Physiol Rep 7, e14139 (2019).

99. Massudi, H. et al. Age-Associated Changes In Oxidative Stress and NAD+ Metabolism In Human Tissue. PLoS ONE 7, e42357 (2012).

100. Rajman, L., Chwalek, K. \& Sinclair, D. A. Therapeutic Potential of NAD-Boosting Molecules: The In Vivo Evidence. Cell Metabolism 27, 529-547 (2018).

101. Connell, N. J., Houtkooper, R. H. \& Schrauwen, P. NAD+ metabolism as a target for metabolic health: have we found the silver bullet? Diabetologia 62, 888-899 (2019).

102. Mills, K. F. et al. Long-Term Administration of Nicotinamide Mononucleotide Mitigates Age-Associated Physiological Decline in Mice. Cell Metabolism 24, 795-806 (2016).

103. Zhang, $\mathrm{H}$. et al. $\mathrm{NAD}^{+}$repletion improves mitochondrial and stem cell function and enhances life span in mice. Science 352, 1436-1443 (2016).

104. Pirinen, E. et al. Pharmacological Inhibition of Poly(ADP-Ribose) Polymerases Improves Fitness and Mitochondrial Function in Skeletal Muscle. Cell Metabolism 19, 1034-1041 (2014).

105. Mouchiroud, L. et al. The NAD+/Sirtuin Pathway Modulates Longevity through Activation of Mitochondrial UPR and FOXO Signaling. Cell 154, 430-441 (2013).

106. Elhassan, Y. S. et al. Nicotinamide Riboside Augments the Aged Human Skeletal Muscle NAD+ Metabolome and Induces Transcriptomic and Anti-inflammatory Signatures. Cell Rep 28, 1717-1728.e6 (2019). 
107. Dollerup, O. L. et al. Nicotinamide riboside does not alter mitochondrial respiration, content or morphology in skeletal muscle from obese and insulin-resistant men. $J$ Physiol 598, 731-754 (2020).

108. Remie, C. M. E. et al. Nicotinamide riboside supplementation alters body composition and skeletal muscle acetylcarnitine concentrations in healthy obese humans. $A m \mathrm{~J}$ Clin Nutr 112, 413-426 (2020).

109. Martens, C. R. et al. Chronic nicotinamide riboside supplementation is well-tolerated and elevates NAD+ in healthy middle-aged and older adults. Nat Commun 9, 1286 (2018).

110. Dolopikou, C. F. et al. Acute nicotinamide riboside supplementation improves redox homeostasis and exercise performance in old individuals: a double-blind cross-over study. Eur J Nutr 59, 505-515 (2020).

111. Crisol, B. M. et al. NAD+ precursor increases aerobic performance in mice. Eur J Nutr 59, 2427-2437 (2020).

112. Kourtzidis, I. A. et al. Nicotinamide riboside supplementation dysregulates redox and energy metabolism in rats: Implications for exercise performance. Exp Physiol 103, 1357-1366 (2018).

113. Das, A. et al. Impairment of an Endothelial NAD+-H2S Signaling Network Is a Reversible Cause of Vascular Aging. Cell 173, 74-89.e20 (2018).

114. Pirinen, E. et al. Niacin Cures Systemic NAD+ Deficiency and Improves Muscle Performance in Adult-Onset Mitochondrial Myopathy. Cell Metab 31, 1078-1090.e5 (2020).

115. Park, J.-S., Davis, R. L. \& Sue, C. M. Mitochondrial Dysfunction in Parkinson's Disease: New Mechanistic Insights and Therapeutic Perspectives. Curr Neurol Neurosci Rep 18, 21 (2018).

116. Radak, Z. et al. Physical exercise, reactive oxygen species and neuroprotection. Free Radic Biol Med 98, 187-196 (2016).

117. Bernardo, T. C. et al. Physical Exercise and Brain Mitochondrial Fitness: The Possible Role Against Alzheimer's Disease. Brain Pathol 26, 648-663 (2016). 


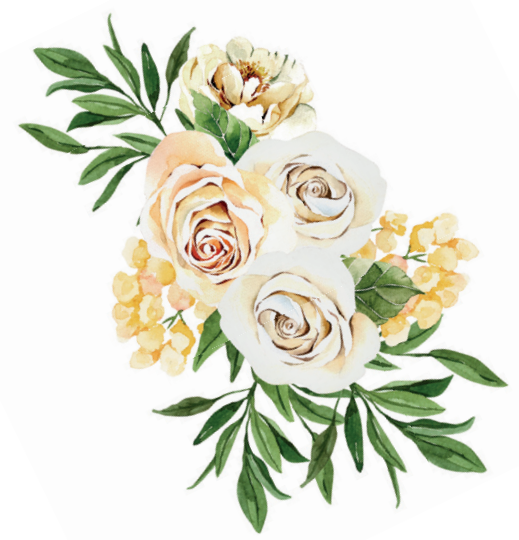

\section{APPENDICES}

Samenvatting

Summary

Sommario

Impact

Dankwoord

About the author

List of publications

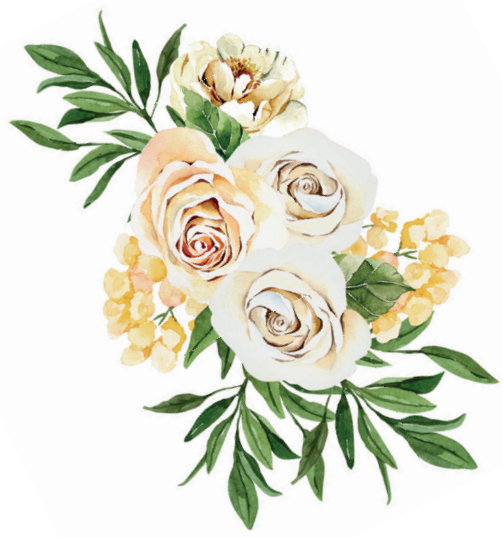




\section{SAMENVATTING}

Spiergezondheid en veroudering: een focus op mitochondriën, metabolisme en fysieke functie.

Mensen leven langer dan ooit tevoren en ondanks de voortdurend verbeterende levensverwachting en gezondheidszorg, is het behoud van een goede levenskwaliteit gedurende deze extra jaren niet vanzelfsprekend. Eén van de meest opvallende processen bij het ouder worden is de progressieve afname van spiermassa en spierkracht. Dit verlies van spierkwaliteit is gaat gepaard met functionele beperkingen en het verlies van onafhankelijkheid in dagelijkse activiteiten, maar ook met metabole stoornissen en een groter risico op chronische ziekten. Eén van de veronderstelde oorzaken van het verlies van spiergezondheid tijdens veroudering is de afname van de mitochondriële capaciteit. Dit proefschrift beoogt een beter inzicht te krijgen in de rol van het mitochondriële metabolisme in de afname van de spiergezondheid tijdens veroudering. Daarbij is tevens aandacht voor verschillende andere factoren die tijdens het verouderingsproces vaak gelijktijdig veranderen, zoals een afname van fysieke activiteit en veranderingen in lichaamssamenstelling. Inzichten in het proces van spierveroudering op fysiologisch en moleculair niveau kunnen bijdragen aan de verdere ontwikkeling van optimale strategieën om ook op oudere leeftijd een goede (spier)gezondheid en levenskwaliteit te behouden.

Mitochondriën zijn geen op zichzelf staande organellen, maar vormen strak gereguleerde, dynamische netwerken. Om te kunnen voldoen aan de fluctuerende energiebehoeftes van de cel worden deze netwerken voortdurend opgebouwd, afgebroken en onderworpen aan strenge kwaliteitscontroles. Om de gepaste morfologie en functie te behouden, kunnen slecht functionerende delen van mitochondriële netwerken worden afgesplitst en afgebroken via een proces dat mitofagie wordt genoemd. Daarnaast kunnen nieuwgevormde mitochondriën fuseren met andere mitochondriën om zo het mitochondriële netwerk aan te passen aan de fysiologische benodigdheden van de cel. Een goede dynamiek van het netwerk is essentieel voor het behoud van een optimale mitochondriële functie. Hoofdstuk 2 bestudeert de beschikbare literatuur over verstoringen in de regulatie van mitochondriële netwerken bij veroudering. Deze literatuurstudie laat zien dat een 
afwijkende mitochondriële dynamiek en verminderde kwaliteitscontrole gerelateerd zijn aan de gezondheid van skeletspieren bij veroudering. De conclusie is ook dat er meer humane studies nodig zijn om de onderliggende mechanismen te begrijpen en nieuwe therapieën te ontwikkelen om de verstoorde mitochondriële dynamiek bij ziekte en veroudering aan te pakken.

Of de afname van de mitochondriële functie tijdens het ouder worden verklaard wordt door de toenemende (chronologische) leeftijd of meer door een leeftijdsgebonden afname in fysieke activiteit is nog onduidelijk. In hoofdstuk $\mathbf{3}$ werd daarom het effect van leeftijd op mitochondriële functie en spiergezondheid onderzocht aan de hand van een gedetailleerde fenotypische karakterisering van jongere en oudere personen met vergelijkbare niveaus van fysieke activiteit. Daarnaast werd de invloed van fysieke activiteit op het verouderingsproces onderzocht door een vergelijking te maken in spierfunctie en spiergezondheid tussen getrainde ouderen, ouderen met een normaal niveau van fysieke activiteit en ouderen met een verminderde fysieke functie. Het vergelijken van deze groepen laat zien dat veroudering geassocieerd is met een afname van de mitochondriële functie, inspanningsvermogen, inspanningsefficiëntie, loopstabiliteit, spierfunctie en insulinegevoeligheid. Opvallend genoeg zien we deze afname in ouderen ondanks dat het fysiek activiteitsniveau vergelijkbaar is met dat van jongeren. Tevens voldoen beide groepen qua activiteit aan de algemene aanbevelingen, namelijk een gemiddelde van 10000 stappen per dag. Enkel een forse verhoging van de fysieke activiteit, door middel van regelmatig en intensief te trainen, blijkt de effecten van veroudering op de mitochondriële functie en spierfunctie tegen te kunnen gaan. Daarnaast onderschrijven de waargenomen correlaties tussen mitochondriële capaciteit en inspanningsefficiëntie en insulinegevoeligheid, de rol van mitochondria bij de afname van de spiergezondheid tijdens veroudering. Deze resultaten identificeren de mitochondria als een potentieel therapeutisch doelwit om spierveroudering tegen te gaan en om de fysieke functie te kunnen behouden.

Om beter te begrijpen waarom de mitochondriële functie afneemt met de leeftijd, werden in hoofdstuk 4 de metabole verschillen in het spierweefsel van de proefpersonen uit hoofdstuk 3 verder onderzocht. Het niveau van 137 metabolieten 
(metabole signaalstoffen) werd gedetecteerd en vergeleken in jongeren, ouderen met een normale fysieke functie, getrainde ouderen en ouderen met een verminderde fysieke functie en 65 metabolieten bleken te veranderen met veroudering. Van de metabolieten die verlaagd waren in ouderen ten opzichte van jongeren, was de afname van nicotinamide adenine dinucleotide $\left(\mathrm{NAD}^{+}\right)$het meest prominent. $\mathrm{NAD}^{+}$is een essentieel metaboliet dat onder andere nodig is voor verschillende cellulaire processen die betrokken zijn bij oxidatieve fosforylering en energieproductie in de mitochondriën. Interessant is dat hoofdstuk 4 ook aantoont dat deze afname in $\mathrm{NAD}^{+}$sterker aanwezig is bij ouderen met een verminderde fysieke functie en dat het $\mathrm{NAD}^{+}$niveau bij fysiek getrainde ouderen bijna volledig vergelijkbaar is met dat van de jongeren. $\mathrm{NAD}^{+}$en andere belangrijke metabolieten van het $\mathrm{NAD}^{+}$metabolisme blijken ook sterk te correleren met de mitochondriële functie, het gemiddeld aantal stappen per dag en de spiergezondheid. Deze resultaten tonen de potentie aan van inspanningstraining om gezond ouder te worden en suggereren een cruciale rol van $\mathrm{NAD}^{+}$hierin.

Een mogelijke manier om het aan veroudering gerelateerde verlies in $\mathrm{NAD}^{+}$tegen te gaan is door middel van de suppletie van $\mathrm{NAD}^{+}$- precursoren via de voeding. In hoofdstuk 5 werd het effect onderzocht van een 32 dagen durende suppletie met $\mathrm{NAD}^{+}$-precursoren (bestaande uit L-tryptofaan, nicotinezuur en nicotinamide) op de mitochondriële functie en spiergezondheid in ouderen met een lagere fysieke functie. In de controle arm namen alle deelnemers gedurende 32 dagen tevens een controlemiddel, zonder $\mathrm{NAD}^{+}$precursoren. Hierbij was niet bekend in welke volgorde zij de $\mathrm{NAD}^{+}$supplementen en het controlemiddel ontvingen. De studieresultaten tonen aan dat de $\mathrm{NAD}^{+}$concentraties in de skeletspier niet significant verschillen in vergelijking met de controleconditie. Echter is de concentratie van het afbraakproduct methyl-nicotinamide significant hoger na suppletie met $\mathrm{NAD}^{+}-$ precursoren wat lijkt te wijzen op een verhoging van het $\mathrm{NAD}^{+}$metabolisme. De mitochondriële respiratie capaciteit blijkt niet te verbeteren door suppletie met NAD+precursoren en de inspanningsefficiëntie en spierfunctie blijven onveranderd. Deze resultaten zijn vergelijkbaar met andere studieresultaten over de werkzaamheid van $\mathrm{NAD}^{+}$-precursoren via de voeding in mensen. Kortom, de inname van extra NAD ${ }^{+}$ precursoren bestaande uit L-tryptofaan, nicotinezuur en nicotinamide verhoogt de 
methylnicotinamide niveaus in de spieren, maar verbetert de mitochondriële capaciteit en spiergezondheid niet in ouderen met een verminderde fysieke functie.

Eén van de meest kritieke complicaties van veroudering is het verlies van evenwichtscontrole en stabiliteit, wat leidt tot een verhoogd risico op vallen en valgerelateerde gevolgen zoals breuken, verdere functionele achteruitgang en immobiliteit. Over het algemeen wordt aangenomen dat spiermassa en kracht essentiële factoren zijn om met grote evenwichtsverstoringen om te gaan. Echter blijkt spierkrachttraining slechts een matig effect te hebben op de evenwichtscontrole. De leeftijdsgebonden afname van spiermassa wordt voornamelijk toegeschreven aan atrofie van type II spiervezels wat leidt tot een afname van de spierkracht. In hoofdstuk 6 onderzochten we of de karakteristieken van de type II spiervezels al dan niet geassocieerd zijn met de evenwichtscontrole van jonge en oudere personen. Om dit te doen werd de dynamische stabiliteit gemeten tijdens een evenwichtsverstoring op een loopband en werden de spiervezels gekarakteriseerd in spierbiopsies van de jongvolwassenen, normaal actieve ouderen, getrainde ouderen en ouderen met een verminderde spierfunctie. Ondanks de grote variatie in spiervezelverdeling, spiermassa en spierkracht over de verschillende groepen, blijken deze spiereigenschappen niet te correleren met de stabiliteit tijdens deze evenwichtsverstoringen. Dit geeft aan dat valpreventie interventies niet enkel zouden moeten focussen op het verbeteren van de kwaliteit van het spierweefsel, maar dat mogelijk ook de andere verouderingsprocessen moeten worden aangepakt om functionele achteruitgang in ouderen te voorkomen.

Op basis van dit proefschrift kunnen we concluderen dat ondanks het handhaven van de aanbevolen hoeveelheid dagelijkse lichaamsbeweging, veroudering gepaard gaat met een afname van de mitochondriële capaciteit, NAD ${ }^{+}$metabolisme, spiergezondheid, loopstabiliteit en fysieke functie. Enkel hogere niveaus van fysieke activiteit, bereikt door regelmatig en intensief te trainen, kunnen de effecten tegen gaan van veroudering op het $\mathrm{NAD}^{+}$metabolisme, mitochondriële functie en spiergezondheid. Een beter begrip van de regulatie van mitochondriële functie en de rol van $\mathrm{NAD}^{+}$metabolisme in de spier bieden mogelijk therapeutische aanknopingspunten om de achteruitgang in fysieke functie tegen te gaan en op een gezonde manier ouder te worden. 


\section{SUMMARY}

\section{Skeletal muscle health in aging: A focus on mitochondria, metabolism, and physical performance}

People are living longer than ever before and despite continuously improving life expectancy and healthcare, maintaining a good quality of life during these extra years is not a matter of course. One of the most striking processes of aging is the progressive decline in muscle mass and strength. This loss of muscle quality is associated with functional limitations and loss of independence in daily activities, as well as metabolic disorders and a greater risk of chronic disease. One of the hypothesized causes of the loss of muscle health during aging is the decrease in mitochondrial capacity. This thesis aims to gain a better understanding of the role of mitochondrial metabolism in the decline of muscle health during aging. Attention is also paid to several other factors that often change simultaneously during the aging process, such as a decrease in physical activity and changes in body composition. Insights into the process of muscle aging on a physiological and molecular level can contribute to the further development of to maintain good (muscle) health and quality of life in old age.

Mitochondria form tightly regulated, dynamic networks. To meet the fluctuating energy needs of the cell, these networks are continuously built, broken down, and subjected to strict quality controls. To maintain appropriate morphology and function, malfunctioning portions of mitochondrial networks can be cleaved off and broken down through a process called mitophagy. In addition, newly formed mitochondria can fuse with other mitochondria to adapt the mitochondrial network to the physiological needs of the cell. Good network dynamics are essential for maintaining optimal mitochondrial function. Chapter 2 examines the available literature on disturbances in the regulation of mitochondrial networks in aging. This literature review shows that aberrant mitochondrial dynamics and impaired quality control are related to skeletal muscle health in aging. It also concludes that more human studies are needed to understand the underlying mechanisms and develop new therapies to address the disrupted mitochondrial dynamics in disease and aging.

Whether the decrease in mitochondrial function during aging is explained by increasing (chronological) age or by an age-related reduction in physical activity is 
still unclear. Therefore, in chapter 3 the effect of age on mitochondrial function and muscle health was investigated by detailed phenotypic characterization of younger and older individuals with comparable levels of physical activity. In addition, the influence of physical activity on the aging process was investigated by comparing muscle function and muscle health between trained older adults, older adults with normal levels of physical activity, and older adults with reduced physical function. Comparing these groups shows that aging is associated with a decrease in mitochondrial function, exercise capacity, exercise efficiency, gait stability, muscle function, and insulin sensitivity. Remarkably, we see this decrease in older adults despite the fact that the physical activity level is comparable to that of young people. Both groups also meet the general recommendations in terms of activity, namely an average of 10,000 steps per day. Only a substantial increase in physical activity, through regular and intensive training, appears to counteract the effects of aging on mitochondrial and muscle function. In addition, the observed correlations between mitochondrial capacity and exercise efficiency, and insulin sensitivity support the role of mitochondria in the decline in muscle health during aging. These results identify the mitochondria as a potential therapeutic target to counteract muscle aging and maintain physical function.

To better understand why mitochondrial function declines with age, in chapter 4 the metabolic differences in the muscle tissue of the subjects from chapter 3 were further investigated. The level of 137 metabolites (metabolic signaling substances) was detected and compared in young people, the older adults with normal physical function, trained older adults, and older adults with impaired physical function, and 65 metabolites were found to change with aging. Of the metabolites decreased in the older adults compared to the young, the decrease in nicotinamide adenine dinucleotide $\left(\mathrm{NAD}^{+}\right)$was most prominent. $\mathrm{NAD}^{+}$is an essential metabolite required for various cellular processes involved in oxidative phosphorylation and energy production in the mitochondria. Interestingly, chapter 4 also shows that this decrease in $\mathrm{NAD}^{+}$is more pronounced in older adults with impaired physical function and that $\mathrm{NAD}^{+}$levels in physically trained older adults are almost completely comparable to that of the young. $\mathrm{NAD}^{+}$and other metabolites of $\mathrm{NAD}^{+}$metabolism have also been found to correlate strongly with mitochondrial function, average steps 
per day, and muscle health. These results demonstrate the potential of exercise training for healthy aging and suggest a crucial role of $\mathrm{NAD}^{+}$in this.

A possible way to counteract the aging-related loss in $\mathrm{NAD}^{+}$is through dietary supplementation of $\mathrm{NAD}^{+}$precursors. In chapter 5, the effect of a 32-day supplementation with $\mathrm{NAD}^{+}$precursors (consisting of L-tryptophan, nicotinic acid, and nicotinamide) on mitochondrial function and muscle health in older adults with lower physical function was investigated. In the control arm, all participants also took a control product, without $\mathrm{NAD}^{+}$precursors, for 32 days. It was not known in which order they received the $\mathrm{NAD}^{+}$supplements and the control. The study results show that the $\mathrm{NAD}^{+}$concentrations in skeletal muscle do not differ significantly compared to the control condition. However, the concentration of the degradation product methyl-nicotinamide is significantly higher after supplementation with $\mathrm{NAD}^{+}$ precursors, which seems to indicate an increase in $\mathrm{NAD}^{+}$metabolism. The mitochondrial respiratory capacity does not appear to be improved by supplementation with $\mathrm{NAD}^{+}$precursors and the exercise efficiency and muscle function remain unchanged. These results are comparable to other study results on the efficacy of dietary $\mathrm{NAD}^{+}$precursors in humans. In short, the intake of additional $\mathrm{NAD}^{+}$precursors consisting of L-tryptophan, nicotinic acid, and nicotinamide increases methylnicotinamide levels in muscles but does not improve mitochondrial capacity and muscle health in older adults with a reduced physical function.

One of the most critical complications of aging is loss of balance control and stability, leading to an increased risk of falls and fall-related consequences such as fractures, further functional decline, and immobility. It is generally believed that muscle mass and strength are essential factors for dealing with significant imbalances. However, muscle strength training appears to have only a moderate effect on balance control. The age-related decrease in muscle mass is mainly attributed to the atrophy of type II muscle fibers leading to a decrease in muscle strength. In chapter 6 we investigated whether or not the characteristics of the type II muscle fibers are associated with balance control in young and older participants. Dynamic stability was measured during a balance disturbance on a treadmill, and muscle fibers were characterized in muscle biopsies from the young adults, normally active older adults, trained older adults, and older adults with impaired muscle function. Despite the large variation in muscle fiber distribution, muscle mass, and muscle strength across 
the different groups, these muscle properties do not appear to correlate with stability during these imbalances. This indicates that fall prevention interventions should not only focus on improving muscle tissue quality but may also need to address other aging processes to prevent functional decline in older adults.

Based on this thesis, we can conclude that despite maintaining the recommended amount of daily physical activity, aging is associated with a decrease in mitochondrial capacity, $\mathrm{NAD}^{+}$metabolism, muscle health, gait stability, and physical function. Only higher levels of physical activity, achieved through regular and intense exercise, can counteract the effects of aging on $\mathrm{NAD}^{+}$metabolism, mitochondrial function, and muscle health. A better understanding of the regulation of mitochondrial function and the role of $\mathrm{NAD}^{+}$metabolism in muscle may provide therapeutic leads to counteract the decline in physical function and age in a healthy way. 


\section{SOMMARIO}

Salute dei muscoli scheletrici nell'invecchiamento: un focus su mitocondri, metabolismo e prestazioni fisiche

Le persone vivono più a lungo che mai e, nonostante il continuo miglioramento dell'aspettativa di vita e dell'assistenza sanitaria, mantenere una buona qualità della vita durante tutti questi anni non è scontato. Uno dei processi più eclatanti dell'invecchiamento, è il progressivo declino della massa muscolare e della forza. Questa perdita di qualità muscolare, è associata a limitazioni funzionali e perdita di indipendenza nelle attività quotidiane, nonché a disturbi metabolici e ad un maggior rischio di malattie croniche. Una delle cause ipotizzate della perdita di salute muscolare durante l'invecchiamento, è la diminuzione della capacità mitocondriale. Questa tesi, mira ad ottenere una migliore comprensione del ruolo del metabolismo mitocondriale nel declino della salute muscolare durante l'invecchiamento. Viene prestata attenzione anche a diversi altri fattori, che spesso cambiano contemporaneamente durante il processo di invecchiamento, come la diminuzione dell'attività fisica e le variazioni della composizione corporea. Approfondimenti sul processo di invecchiamento muscolare a livello fisiologico e molecolare, possono contribuire all'ulteriore sviluppo di strategie ottimali per mantenere una buona salute (muscolare) e una buona qualità di vita in età avanzata.

I mitocondri, sono reti dinamiche strettamente regolate. Per soddisfare le fluttuazioni del fabbisogno energetico della cellula, queste reti vengono continuamente costruite, scomposte e sottoposte a severi controlli di qualità. Per mantenere una morfologia e una funzione appropriate, le parti malfunzionanti delle reti mitocondriali possono essere scisse e scomposte attraverso un processo chiamato mitofagia. Inoltre, i mitocondri di nuova formazione, possono fondersi con altri mitocondri per adattare la rete mitocondriale ai bisogni fisiologici della cellula. Una buona dinamica della rete è essenziale per mantenere una funzione mitocondriale ottimale. II capitolo 2 , esamina la letteratura disponibile sui disturbi nella regolazione delle reti mitocondriali nell'invecchiamento. Questa revisione della letteratura, mostra che le dinamiche mitocondriali aberranti e il controllo di qualità alterato, sono correlati alla salute del muscolo scheletrico nell'invecchiamento. Si afferma inoltre, che sono necessari ulteriori studi sull'uomo per comprendere i meccanismi sottostanti e sviluppare nuove 
terapie per affrontare le dinamiche mitocondriali interrotte nella malattia e nell'invecchiamento.

Non è ancora chiaro se la diminuzione della funzione mitocondriale durante l'invecchiamento sia spiegata dall'aumento dell'età (cronologica) o, piuttosto, da una diminuzione dell'attività fisica correlata all'età. Pertanto, nel capitolo 3 , è stato studiato l'effetto dell'età sulla funzione mitocondriale e sulla salute muscolare, mediante una dettagliata caratterizzazione fenotipica di individui più giovani e più anziani con comparabili livelli di attività fisica. Inoltre, è stata studiata l'influenza dell'attività fisica sul processo di invecchiamento, confrontando la funzione muscolare e la salute muscolare tra anziani che fanno spesso sport, anziani con normali livelli di attività fisica e anziani con funzione fisica ridotta. II confronto di questi gruppi, mostra che l'invecchiamento è associato a una diminuzione della funzione mitocondriale, della capacità di esercizio, dell'efficienza dell'esercizio, della stabilità dell'andatura, della funzione muscolare e della sensibilità all'insulina. Sorprendentemente, vediamo questa diminuzione negli anziani, nonostante il fatto che il livello di attività fisica sia paragonabile a quello dei giovani. Entrambi i gruppi, soddisfano anche le raccomandazioni generali in termini di attività, ovvero una media di 10.000 passi al giorno. Solo un sostanziale aumento dell'attività fisica, attraverso un allenamento regolare e intensivo, sembra contrastare gli effetti dell'invecchiamento sulla funzione mitocondriale e muscolare. Inoltre, le correlazioni osservate tra capacità mitocondriale, efficienza dell'esercizio e sensibilità all'insulina, supportano il ruolo dei mitocondri nel declino della salute muscolare durante l'invecchiamento. Questi risultati, identificano i mitocondri come un potenziale bersaglio terapeutico, per contrastare l'invecchiamento muscolare e mantenere la funzione fisica.

Per capire meglio perché la funzione mitocondriale diminuisce con l'età, nel capitolo 4 sono state ulteriormente studiate le differenze metaboliche nel tessuto muscolare dei soggetti del capitolo 3. II livello di 137 metaboliti (sostanze di segnalazione metabolica), è stato rilevato e confrontato nei giovani, negli anziani con funzione fisica normale, negli anziani allenati e negli anziani con funzione fisica compromessa e, 65 metaboliti, sono stati trovati a cambiare con l'invecchiamento. Dei metaboliti diminuiti negli anziani rispetto ai giovani, la diminuzione della nicotinamide adenina dinucleotide $\left(\mathrm{NAD}^{+}\right)$è stata la più notevole. $\mathrm{NAD}^{+}$, è un metabolita essenziale 
richiesto per vari processi cellulari coinvolti nella fosforilazione ossidativa e nella produzione di energia nei mitocondri. È interessante notare, che il capitolo 4 mostra anche che questa diminuzione di $\mathrm{NAD}^{+}$è più pronunciata negli anziani con funzione fisica compromessa e che i livelli di NAD+ negli anziani allenati fisicamente, sono quasi completamente paragonabili a quelli dei giovani. Ė stato anche scoperto che il $\mathrm{NAD}^{+}$e altri metaboliti del metabolismo del $\mathrm{NAD}^{+}$sono fortemente correlati con la funzione mitocondriale, i passi medi al giorno e la salute muscolare. Questi risultati dimostrano il potenziale dell'esercizio fisico, per un invecchiamento sano e suggeriscono un ruolo cruciale del $\mathrm{NAD}^{+}$in questo.

Un modo possibile per contrastare la perdita di NAD ${ }^{+}$correlata all'invecchiamento, è attraverso l'integrazione alimentare di precursori di $\mathrm{NAD}^{+}$. Nel capitolo 5 , è stato studiato l'effetto di un'integrazione di 32 giorni con precursori NAD+ (costituiti da Ltriptofano, acido nicotinico e nicotinamide), sulla funzione mitocondriale e sulla salute muscolare negli anziani con funzione fisica inferiore. In una delle fasi, tutti i partecipanti hanno anche assunto un prodotto di controllo, senza precursori NAD ${ }^{+}$ per 32 giorni. Non era noto in quale ordine ricevessero gli integratori $\mathrm{NAD}^{+}$e i prodotti di controllo. I risultati dello studio, mostrano che le concentrazioni di NAD ${ }^{+}$ nel muscolo scheletrico, non differiscono significativamente rispetto alla condizione di controllo. Tuttavia, la concentrazione del prodotto di degradazione metilnicotinammide è significativamente più alta dopo l'integrazione con precursori di $\mathrm{NAD}^{+}$, il che sembra indicare un aumento del metabolismo del NAD ${ }^{+}$. La capacità respiratoria mitocondriale, non sembra essere migliorata dall'integrazione con precursori NAD+ inoltre, l'efficienza dell'esercizio e la funzione muscolare rimangono invariate. Questi risultati sono paragonabili ai risultati di altri studi sull'efficacia dei precursori $\mathrm{NAD}^{+}$nella dieta nell'uomo. In breve, l'assunzione di precursori NAD ${ }^{+}$ aggiuntivi costituiti da L-triptofano, acido nicotinico e nicotinamide aumenta i livelli di metilnicotinamide nei muscoli, ma non migliora la capacità mitocondriale e la salute muscolare negli anziani con una funzione fisica ridotta.

Una delle complicanze più critiche dell'invecchiamento, è la perdita del controllo dell'equilibrio e della stabilità, che porta ad un aumento del rischio di cadute e delle conseguenze ad esse correlate, come fratture, ulteriore declino funzionale e, qualche volta, immobilità. Si ritiene generalmente, che la massa muscolare e la forza siano fattori essenziali per affrontare squilibri significativi. Tuttavia, l'allenamento 
della forza muscolare sembra avere solo un effetto moderato sul controllo dell'equilibrio. La diminuzione della massa muscolare legata all'età, è principalmente attribuita all'atrofia delle fibre muscolari di tipo II che porta ad una diminuzione della forza muscolare. Nel capitolo 6, abbiamo studiato se le caratteristiche delle fibre muscolari di tipo II, sono associate o meno al controllo dell'equilibrio in soggetti giovani e anziani. La stabilità dinamica, è stata misurata durante un disturbo dell'equilibrio su un tapis roulant e le fibre muscolari sono state caratterizzate in biopsie muscolari di giovani adulti, anziani normalmente attivi, anziani allenati e anziani con funzione muscolare compromessa. Nonostante la grande variazione nella distribuzione delle fibre muscolari, nella massa muscolare e nella forza muscolare tra i diversi gruppi, queste proprietà muscolari non sembrano essere correlate alla stabilità durante questi squilibri. Ciò indica che gli interventi di prevenzione delle cadute, non dovrebbero concentrarsi solo sul miglioramento della qualità del tessuto muscolare, ma potrebbero anche dover affrontare altri processi di invecchiamento per prevenire il declino funzionale negli anziani.

Sulla base di questa tesi, possiamo concludere che, nonostante il mantenimento della quantità raccomandata di attività fisica quotidiana, l'invecchiamento è associato a una diminuzione della capacità mitocondriale, del metabolismo $\mathrm{NAD}^{+}$, della salute muscolare, della stabilità dell'andatura e della funzione fisica. Solo livelli più elevati di attività fisica, raggiunti attraverso un esercizio fisico intenso e regolare, possono contrastare gli effetti dell'invecchiamento sul metabolismo $\mathrm{NAD}^{+}$, sulla funzione mitocondriale e sulla salute muscolare. Una migliore comprensione della regolazione della funzione mitocondriale e del ruolo del metabolismo $\mathrm{NAD}^{+}$nel muscolo, può fornire indicazioni terapeutiche per contrastare il declino della funzione fisica e dell'età in modo sano. 


\section{IMPACT}

What is the main objective of the thesis, and what are the most important results and conclusions?

Between 1990 and 2019, the number of people older than 80 has tripled, and this number is expected to triple again by 2050 (1). This enormous accomplishment of increasing life expectancy illustrates the remarkable advances achieved in biomedicine and healthcare but comes at a cost though, since many diseases such as type 2 diabetes and cardiovascular diseases are more prevalent at an older age (2). Moreover, in proportion, there are increasingly more older adults in the population than young individuals, an imbalance known as the 'silver tsunami,' which implicates increased healthcare costs and significant social challenges. If we consider the aging human body, skeletal muscle is prominently affected. Ageinduced loss of lean muscle mass and function is known as sarcopenia and leads to disabilities and increased dependency and care needs. A better understanding of the aging process itself, rather than focusing on age-associated disease, can help to develop strategies to target the aging process and maintain people's health during those extra years of life.

In the cells of the human body, the mitochondria are organelles that maintain cellular energy levels necessary for all vital processes. As a result, mitochondria are also known as the cell's powerhouses. In muscle cells, healthy mitochondria regulate various metabolic processes and provide energy for muscle contractions. It is well documented that with aging, muscle mitochondrial function declines, which is paralleled by a loss in muscle strength and mass. Importantly, this reduction in muscle health translates into a loss of physical independence and an increased risk for metabolic disease. If and how age-related alterations in mitochondrial function and muscle health (causally) relate to each other is however not well established in humans. This thesis aimed to better understand the aging process in skeletal muscle by investigating the role of mitochondrial metabolism in the age-related loss of physical performance.

It has become increasingly clear that mitochondria do not operate as individual organelles but physically and functionally connect into dynamic networks. These networks are constantly built, restored, and subjected to quality control mechanisms 
to assure efficient mitochondrial function. Growing evidence indicates that altered mitochondrial dynamics and impaired quality control influence skeletal muscle health in aging and metabolic disease. In chapter 2 this evidence was reviewed in a literature study on the role of abnormal mitochondrial network dynamics in the agerelated loss of muscle health. However, more - especially human - studies are needed to understand the implications and underlying mechanisms in order to develop new therapeutics to target mitochondrial dynamics in human disease. An important first step in this context is to develop suitable techniques to study these dynamic processes properly.

To better understand the relationship between skeletal muscle mitochondrial metabolism and muscle health, we, in chapter 3, extensively measured mitochondrial capacity and muscle function in young and older adults. We found that aging is associated with a decline in mitochondrial capacity, muscle health, and physical function. Interestingly, this decline was observed in older adults despite the fact that they performed more than 10000 steps-per-day, which is above the general physical activity recommendations. Since we also studied trained older adults in this study, we could also show that only a further increase in physical activity levels, achieved through regular high-intensity exercise training, was able to prevent the effects of aging on muscle health. Furthermore, we found a correlation between mitochondrial function and muscle health, indicating that maintaining mitochondrial function at older age is important to promote healthy aging.

To further understand why mitochondrial function declines when we age, we next studied the muscle tissue of the young and older participants and compared their metabolic profiles, using untargeted metabolomics (chapter 4). The most prominent change we observed was an age-related decline in $\mathrm{NAD}^{+}$, an essential metabolite that is needed in the mitochondria to generate energy. Interestingly, this decline in $\mathrm{NAD}^{+}$was exacerbated in older individuals with a lower physical function and was nearly entirely prevented in the exercise-trained older adults. $\mathrm{NAD}^{+}$also strongly correlated to the average steps-per-day and mitochondrial and muscle functioning, demonstrating the importance of exercise to age healthier and suggesting a crucial role of $\mathrm{NAD}^{+}$therein. 
A strategy to potentially alleviate the age-related depletion of $\mathrm{NAD}^{+}$in the mitochondria is by stimulating $\mathrm{NAD}^{+}$synthesis through nutritional supplementation of $\mathrm{NAD}^{+}$precursors. In this context, chapter 5 explored the effectiveness of $\mathrm{NAD}^{+}$ precursors supplementation (consisting of tryptophan, vitamin B3, and nicotinamide) on $\mathrm{NAD}^{+}$levels, mitochondrial function, and muscle health in older adults with reduced physical function. Nevertheless, the extra intake of these $\mathrm{NAD}^{+}$precursors appeared not to be sufficient to boost mitochondrial function and failed to improve physical function.

One of the most critical consequences of age-related decrease in physical function is the loss of balance control leading to an increased risk of falls and fall-related effects such as fractures, further functional decline, immobility, and causal mortality. Therefore, fall prevention therapy is essential to promote healthy aging. The reduction in muscle strength and mass is assumed to be the main reason for the age-related loss in the ability to cope with balance disturbances. Nevertheless, in chapter 6, no strong correlations were observed in young and older adults between muscle characteristics and balance control. These results indicate that fall prevention therapy in older individuals should not only focus on improving muscle quality, but a multi-domain intervention might be necessary.

Taken together, results from this dissertation indicate the importance of mitochondrial function and $\mathrm{NAD}^{+}$metabolism in muscle aging and suggest that physical exercise training remains the most powerful tool to improve mitochondrial function and to age in a healthy way. A better understanding of the role of $\mathrm{NAD}^{+}$in muscle metabolism and the mitochondrial quality control mechanism can reveal promising therapeutic targets to promote healthy aging.

\section{What is the contribution of the research results to science and society?}

The studies described in this thesis add to the existing knowledge on changes that occur in skeletal muscle while human age. With this new knowledge, we add some pieces to the puzzle and contribute to the clarification of the complex process of aging. Our results may help to identify new targets and approaches in order to prevent age-related disability and help people live a long healthy life. 
Due to the vital role of skeletal muscle in daily life activities, the deterioration of muscle mass and function (sarcopenia) leads to a decreased quality of life, increased hospitalization rates and the loss of independence. Over the coming decades, the prevalence of sarcopenia is expected to increase drastically in Europe, and these trends will not only be a burden for the older population. They will impose a tremendous financial load on society as a whole in terms of increased healthcare costs, which are expected to double for European countries such as The Netherlands (3). To make healthcare affordable and preserve a good quality of life, it is crucial to maintain people's health and physical independence at older age. A better understanding of the aging process itself can help us identify strategies to promote healthy aging and significantly impact society.

\section{To whom are the research results relevant?}

The research results of this thesis are relevant to different stakeholders. First of all, these results are of interest to researchers working in the field of aging and agingrelated diseases, as they contribute to a better understanding of how skeletal muscle metabolism and function change as we age. With this knowledge, researchers can investigate new targets and explore new avenues aimed at growing old in a healthy way. The new insights into the relationship between impaired muscle health and mitochondrial metabolism are also interesting to the food industry, as mitochondrial metabolism may be sensitive to nutritional intervention. This opens up new opportunities to develop nutritional strategies to prevent the age-related loss of physical function. The research results also support clear benefits of exercise training for older adults, which allow better recommendations, explanations, and motivations on how to maintain a healthy lifestyle. These recommendations can be adopted by the government and/or healthcare in general. Finally, these studies are of interest to the general public as they provide leads on how to preserve a healthy muscle function and a high quality of life while aging.

Several communication strategies are applied to inform the various relevant stakeholders. The results are used for original scientific articles which have been submitted to international, peer-reviewed journals. Once these articles are published, the knowledge from these studies is shared with scientists worldwide. The studies 
have also been presented at several conferences and symposia, which increases the visibility of the results and thereby contributes to new insights and ideas for future research. In addition, during regular expert meetings and project-based seminars, the results have been shared regularly with the industrial partners and collaborating universities within the TIFN Mitochondrial Health project. Being part of this TIFN consortium enhanced collaborations between academia and industry, thereby fostering translational research. Finally, the results can be communicated during general lectures to increase public awareness of the importance of healthy aging

\section{REFERENCES}

1. United Nations. World Population Ageing 2019. Department of Economic and Social Affairs, Population Division (2020).

2. Prince, M. J. et al. The burden of disease in older people and implications for health policy and practice. The Lancet 385, 549-562 (2015).

3. European Commission, Directorate-General for Economic and Financial Affairs, EC, \& Economic Policy Committee of the European Communities. The 2015 ageing report: economic and budgetary projections for the 28 EU Member States (2013-2060). (Publications Office, 2015). 


\section{DANKWOORD}

En jawel, daar is hij dan, meer in zicht dan ooit: de eindstreep! Dit promotietraject voelde voor mij aan als een lange marathon met wat struikelblokken en doorbijt momenten maar ook veel goede coaches en trouwe supporters die me hebben geholpen dit doel te bereiken. Zonder deze mensen zou ik de eindstreep niet over zijn geraakt en ik wil dan ook de resterende adrenaline van deze eindspurt gebruiken om deze mensen te bedanken.

Ik kan me de dag van mijn sollicitatie nog goed herinneren. De positie leek me enorm interessant en het zou ook betekenen dat ik na 2 jaren Milaan terug dichter bij mijn familie zou komen wonen. Mijn trouwe supporters kwamen daarom ook mee naar de sollicitatie. Ik was best zenuwachtig en eens aangekomen bij de universiteitssingel 50 (dankzij het goede zoekwerk van mama!), ging mijn broer voor de zekerheid even mee naar binnen om me niet als een kip zonder kop verloren te laten lopen. Toen ik uiteindelijk aankwam op het juiste secretariaat (bedankt broer!), werd ik ontvangen door twee vriendelijke dames, Désirée en Yolanda. Ik vertelde dat ze ik zenuwachtig was omdat ik deze PhD positie heel erg graag zou krijgen. Lieve Yolanda en Désirée, dat was de eerste van nog vele andere keren dat jullie mij wisten gerust te stellen en mij uit de nood hebben geholpen. Heel erg bedankt voor al jullie ondersteuning, betrokkenheid en lieve attenties.

Joris, ik was enorm blij toen ik een paar dagen na mijn sollicitatie van jou het mailtje kreeg met het goede nieuws dat ik in september 2016 welkom was aan de start van dit PhD avontuur in de DMRG-groep. Van het begin en tot met het einde stond je me bij met raad en daad. Je was een fijne begeleider die niet enkel de focus lag op goede onderzoek prestaties maar ook snel ontdekte hoe ik als persoon in elkaar zit. Je begreep dat nee-zeggen niet één van mijn specialiteiten was en we hebben vaak samengezeten om na te gaan hoe ik meer gefocust en met voldoende energie de eindstreep zou kunnen halen. Dat liep niet altijd even vlotjes maar ik ben heel blij met het eindresultaat. Je kritische input maar ook positieve kijk hebben me helpen groeien als wetenschapper en als persoon en daar ben ik je enorm dankbaar voor. Ik wens je nog veel geluk toe.

Patrick, ook jouw inzet was essentieel tijdens mijn promotietraject. Ik bewonder het enorm hoe goed en efficiënt jij bent in het opvolgen en sturen van de vele 
verschillende projecten in DMRG. Jouw kritische blik op de data en manuscripten en je wetenschappelijke inzichten waren erg waardevol en leerrijk. Ik wens jou en de DMRG-groep nog veel succes toe.

Riekelt, heel fijn dat jij ook deel uitmaakt van het promotieteam. Ik vond het enorm interessant om met jou onderzoeksgroep te mogen samenwerken. Het was leuk te zien hoe de samenkomst van verschillende expertises effectief tot mooie resultaten kan leiden. Ik heb hier veel uit geleerd en wil je bedanken voor je enthousiasme en waardevolle feedback tijdens mijn promotietraject. Ik wens jou en je onderzoeksgroep nog veel succes en nog meer fijne samenwerkingen met Maastricht toe!

To the assessment committee and members of the corona: Stef Kremers, Annemie Schols, Jaap Keijer, Paul Coen, and Tanja Adam, thank you for taking the time to read and review my thesis and to be present during my dissertation.

Georges, although we only saw each other during online meetings, it was a great pleasure for me to work together with you on the Mitochondrial health metabolomics. Your scientific enthusiasm is contagious! Our collaboration extended the Mitohealth project with fascinating new insights and beautiful figures. I am pleased I can complete my thesis with these results. Many thanks for all your effort. Rubén, Bauke and Michel, I also want to thank you for all the energy and enthusiasm you put into this collaboration. It is impressive how well you manage to work with these complex metabolomic data sets. I wish you guys all the best!

Chris, thank you for our pleasant collaboration on the different projects in this thesis. Your ideas, effort, and the many many Caren measurements enhanced the uniqueness of our data set! I learned a lot from working with you and appreciate how you manage to report your scientific work as transparent as possible. It is impressive how many ideas and insights you have! Wouter, also, thank you for helping us out with the Caren measurements. Kenneth, thank you for your support and input during our collaboration! 
Ciarán, many thanks for all your help during my $\mathrm{PhD}$. It was a pleasure to have you on board the Mitohealth project. It is impressive how many ideas and how much knowledge you have. I enjoyed our scientific discussions, coffee breaks, pub quizzes, whiskey tastings, movement cooperation, and the magic hill in Dundalk. Thank you for your critical and valuable input on the manuscripts and your effort during the many clamps. I wish you all the best, some extra sleep sometimes, and good luck with your career.

Vrijwel de meest gedreven deelnemers aan deze marathon waren de vrijwilligers die zich met volledige overtuiging opgaven om deel te nemen aan dit intensieve onderzoek. Jullie toewijding en inspanningen tijdens de vele testen gaf ons de kans deze unieke studie opzet uit te bouwen, met veel interessante en verassende inzichten als resultaat. Het was enorm leuk jullie bij elke test steeds een beetje beter te leren kennen. Bedankt voor jullie aanstekelijke enthousiasme en mooie levenswijsheden!

Ook wil ik dank jullie wel zeggen aan de receptie (het onthaal voor de Vlamingen) van Universiteitssingel 50. Jullie weten onze deelnemers steeds met een vriendelijke glimlach en de nodige uitleg te ontvangen en dat is enorm fijn. Hans, bedankt voor al je hulp!

Matthijs, ook jouw inzichten hebben me geholpen bij het uitvoeren van dit onderzoek en het interpreteren van de resultaten. Ik kan me nog erg goed herinneren dat je door de gangen riep: Oh no, what did we hire!? Toen je hoorde Rodrigo en ikzelf beide waren flauwgevallen tijdens onze eerste oefensessie bloedprikken (oeps). Jouw humor, betrokkenheid, en positieve ingesteldheid zijn van grote waarde voor de DMRG-groep. Ik wens je nog veel succes en vooral vele leuke fietstochtjes toe!

Lena, wat was ik blij toen je aan boord sprong van het Mitohealth project! Jij hebt me enorm veel geleerd, van het aanvragen van ethische toetsing tot $\mathrm{VO}_{2}$-max testen en zoveel meer. Bedankt voor al je hulp en steun en de vele MR-scans die je voor de studie hebt uitgevoerd (ik ben vraag me af hoe het gaat met het 'op en neer' gewicht). Ik wens je nog veel geluk. Esther, bedankt voor jouw onmisbare hulp met de clamp data! 
Yvonne, jij hebt me enorm veel geholpen bij het uitvoeren, de analyses en het afronden van de studie, bedankt daarvoor! Ik heb veel van jou geleerd! Ook wil ik graag Axel, Kim, Pandi en Marlies bedanken voor jullie hulp met de vele MR-scans. Heel fijn dat we ook op jullie konden rekenen! Julian en Vera, bedankt voor jullie inzet bij de MR-metingen en data-analyse. Jullie expertise vormt een grote meerwaarde voor de onderzoeken in de DMRG-groep.

Gert, Esther, en Johanna, jullie werk is in alle onderzoeken binnen DMRG onmisbaar! Enorm bedankt voor het de vele vele biopten en plasma samples die jullie hebben verwerkt voor celkweek, western Blot, oxygraaf metingen, vezeltypering en nog veel meer! Ook bedankt voor het typeren van oneindig veel spiervezels en Johanna bedankt voor me de kneepjes van het oxygraven aan te leren (ik mis die schaapjes soms wel). Jullie hebben me vaak uit de nood geholpen en er was altijd tijd voor een gezellig babbeltje. Het was heel fijn jullie kantoor buren te mogen zijn!

Jeroen, heel erg bedankt voor jouw hulp bij het uitwerken van de Activ-pal data. Ook bedankt voor jouw enthousiasme en uitmuntende BBQ-skills, die kwamen de groep zeker ten goede! Het was leuk je collega te zijn en ik hoop dat je binnenkort nog eens langskomt op de fiets met kleine Jasper!

Bas, dankjewel voor jouw medisch advies tijdens de studies! Niels, bedankt om steeds klaar te staan met raad en daad en bedankt voor de talloze clamps en spierbiopten. Ook bedankt aan Edmundo (muchas gracias!), Vera, Yvo, en Michiel voor jullie tijd en inspanning bij de vele spierbiopten. Jullie zijn onmisbaar in de groep en ik kan me inbeelden dat het niet altijd makkelijk is betrokken te zijn bij zoveel verschillende studies. Desondanks wisten jullie steeds de proefpersonen gerust te stellen met jullie nauwkeurige handelingen, duidelijke uitleg en goede zorgen. Dank jullie wel!

Nynke, nog voor de start van ons promotietraject werd ons verteld dat wij kamergenootjes zouden worden. We mochten samen op Oroboros-cursus naar Oostenrijk en dat was een zeer mooi begin! Het was heel leuk je kantoorgenootje te zijn. Dank je wel voor alle steun en gezelligheid. Ik wens jou en Casper nog veel geluk en succes toe op jullie nieuwe avontuur! 
Froukje, ik was enorm blij toen jij Fort-Belgium kwam versterken! Tijdens jouw eerste week gingen we opzoek naar jouw pasje dat je vergat in je MRUM-jasje dat al vertrokken was naar het washok. Vanaf toen was ik er helemaal van overtuigd dat ik het heel goed zou gaan vinden met jou en je heerlijke, Belgische efficiëntie. We hebben elkaar nog vaker uit de nood geholpen (vergeten sleutels, verloren pasjes, gesloten loopbruggen, dubbel geboekte proefpersonen, presentatie-stress, nood aan pepernoten, nood aan paaseitjes en nood aan Aperollekes) en daar kijk ik met veel plezier op terug. Dank je wel voor alles!

Carlijn, je talent voor organisatie hebben me op vele vlakke wegwijs gemaakt in uitvoeren van humaan onderzoek en in het afwerken van mijn thesis. Dankzij jou vond ik nu ook de weg tot de onderzoeksgroep van Anesthesie en daar ben ik heel erg blij mee! Enorm bedankt voor alle hulp en aanmoedigingen (nie plooien eh!) en nog heel veel succes bij Medtronic!

Anne, Froukje en Carlijn, mijn favoriete hardloopvriendjes! Vooral tijdens de laatste loodjes van deze marathon hebben jullie mij over de streep geholpen. Toen Covid kwam zijn onze loopafspraakjes enorm hard gestegen in frequentie en dat was voor mij heel fijn. De leuke routes, mooie natuur, avontuurlijke klimmetjes, klaagrondjes, en fijne gesprekken doen me steeds enorm veel deugd. Bedankt voor jullie lieve steun, jullie indrukwekkende navigatie vaardigheden, jullie luisterende oren en jullie goede raad. Anne en Vera, het was heel leuk om samen met jullie en te trainen voor onze eerste, echte marathon! Anne, ook wil ik jou bedanken voor je onvergetelijke kazou-concert, prachtig! Vera en Kay, ik kijk ernaar uit jullie aanstekelijke lach weer te kunnen horen tijdens onze loopjes! Of nog eens een wijntje doen is ook goed! Ik wens jullie veel geluk en een mooie carrière toe en ik wens mezelf nog vele leuke loopjes en reisjes toe met jullie!

Charlotte, we hebben vaak zitten klagen tegen elkaar over de struggels van het PhD leven (heel zwaaar), maar gelukkig maken fijne wandelingen, free-runnen en lekkere wijntjes veel goed. Bedankt je steun en voor de vele keren dat je zo lekker voor me hebt gekookt! Nog veel succes met het afronden van je PhD, je kan het!

Verder wil ik iedereen van de DRMG-groep bedanken. Dank jullie wel voor de gezelligheid, de helpende handen en de fijne samenwerkingen. Het was fijn en leerrijk deel uit te maken van deze groep en jullie hebben allemaal op één of andere manier bijgedragen aan dit boekje! 
Ook wil ik de studenten bedanken die me tijdens hun master stage geholpen hebben bij het onderzoek; Pascal, Michelle, Daniel, Lina, en Collin. Bedankt voor de jullie inzet en enthousiasme. Ik wens jullie veel succes toe!

Aan alle leden van VBW en HB: bedankt voor al jullie hulp en de prettige samenwerking. Een grote dank aan Harry, Loek, Marc, Paul, Stephanie, Gabby en Wendy voor alle MRUM en technische ondersteuning. Harry, dank je wel om ook het thuiswerken mogelijk te maken! Stephanie, zo fijn dat jij van aanpakken weet. Bedankt voor je hulp en heerlijke enthousiasme!

Ik wil ook graag de financiële steun van TiFN en NWO bedanken waardoor ik dit onderzoek heb kunnen doen. Het was erg leerrijk om deel uit te mogen van het TiFN consortium met zowel academische als industriële experten en ik wil dan ook alle partners bedanken voor het delen van hun expertise en voor de kritische discussies tijdens onze meetings. Johan, Marjolein, en Jan, bedankt voor jullie waardevolle input op de manuscripten. Bart, Pol, en Carolien het was leuk om samen met jullie collega-promovendi in dit project te zitten en ik wens jullie het nog veel geluk toe.

Also, many thanks to the doctors of the Republic of Juanfrasya! Juanfra and Asya, 6 years ago we met each other years by coincidence in Milan and ever since it has been amazing to be your friend. I am so happy you also moved to the Netherlands, and it is always so lovely to meet you! You guys know hard it is to do a PhD, moeilijk, moeilijk moeilijk! Thank you for your friendship, good advice, and encouragement. At the moment that I'm writing this, I'm looking forward to celebrating together with you your wedding in Rotterdam! I hope you can see your family soon and I wish you cuties pies all the best and a fantastic honeymoon in the Galapagos!

Dank je wel also to Evelyn and Juls! It was great to get to know you guys during your Dutch adventure, and I enjoyed the many moments of fun and special beers we had together. Evelyn, besides being a great colleague, you became a good friend, and I miss our magic carpet bike rides! Thank you so much for your support and friendship! It is a pity that we could not join your wedding, but it made me so happy to see you guys dancing and celebrating! I am looking forward to visiting you in Australia and meeting little Ranga!! I wish you all the best! Veel liefs en tot snel! 
Lieve Jan en Helma, ik vind het heel fijn dat jullie onze buren zijn. Bedankt voor al jullie hulp, interesse, lieve attenties en voor de goede zorgen voor ons huisje en onze plantjes. Ik wens jullie veel geluk toeben nog vele leuke reisjes naar Italië. Lieve Lenny en Carl, bedankt voor alle gezelligheid, de pizza's van Maurizio en de waarschuwingen voor het hoge water! Ik woon hier heel graag!

Dank je wel lieve Bente, jij leerde me de hilariteit van bessen en celorganellen en streven for life!! Anne, Melanie en An-Sofie jullie zijn van beach-beige goud! Bedankt voor jullie spandoeken op het vliegveld, de vele verrassingsfeestjes en zalige (300\%) weekendjes. Onze therapeutische slappe lachbuien zijn opkikkering verzekerd en als ik de lotto won dan zou ik niet het interieur van de Spar veranderen of het te laat door hebben, nee, dan zou ik alvast een kamer gaan boeken in ons toekomstig bejaardentehuis. An-Sofie, kei-hell dank je wel voor al de tijd en moeite die je stak het ontwerp en het drukken van deze thesis. Jij zit vol talent en ik ben echt heel blij met het einderesultaat! Ik heb het heel erg getroffen met jullie als vriendinnen! Ik wens jullie het allerbeste en nog heel veel vriendenboekjes toe!!

Marilux, dr. Letizia!! Grazie mille di tutto, sei fantastica! Poi, grazie mille alla mia bellissima famiglia italiana: Anna, Gianuzzo, Chiara, Giuseppe, Giulia Valentina, Davide, Chiara e Giuseppe (brakerke), senza dimenticare anche Pino, Enza, Claudio, Salvo e Riccio. Siete toppu fuuaitti! Dal primo incontro, anche senza capire una parola in italiano (tranne mangia mangia), mi avete fatto sentire come un pesce dentro l'acqua nella vostra bella Sicilia, perché la vostra ospitalità ed il vostro amore va oltre le parole! Sicuramente non è sempre facile che vostro Bettino stia qua in Olanda, ma vi voglio ringraziare per esserci stati sempre vicini nonostante la distanza. È sempre un gran piacere rivedervi e spero di festeggiare questo dottorato presto anche con voi. Sapete di essere sempre i beneventi nella nostra casuccia. Grazie per il vostro incorragiante supporto, vi auguro tutto il bene ed ancora tantissimi bei momenti insieme.

Lieve oma, ik weet dat jij heel veel boeken hebt en dat jij je boeken enorm koestert. Ik ben er zeker van dat dit boekje je trots maakt op je poezewoefke en ik hoop een plekje in je exclusieve boekenkast te mogen bemachtigen. Ik heb enorm veel bewondering voor jou. Bedankt voor alles! 
En dan is het nu tijd om de lieve, leuke Grevies te bedanken. Tom, Sarina, An, Dennis, ik voel met echt een gelukzakske met jullie als (schoon)broers en (schoon)zussen. Dank jullie wel voor de ontelbare dingen die jullie doen voor mij. Opgroeiend als ruziënde kinderen had ik nooit gedacht dat we het ooit zo goed met elkaar zouden kunnen vinden maar het tegengestelde is waar en ik vind het heerlijk! Tom ik kijk enorm uit naar een vervolg op onze Rock Werchter avonturen en An hou je koffertje maar alvast klaar voor Sevilla! Lieve mama en papa, zonder jullie zou ik niet staan waar ik nu sta en niet zijn wie ik nu ben. Ik heb heel veel bewondering voor jullie en wil jullie heel erg bedanken voor jullie warme liefde en eeuwige steun. Ik ben heel blij jullie weer dicht bij mij te hebben want zonder mijn lieve coach en trouwste supporter was ik hier niet geraakt. Ik zie jullie allemaal enorm graag en wens jullie een mooie toekomst toe met nog vele gezellige Grevie-gelukjes. Laten we snel nog eens gaan wandelen of fietsen want zoals mama het mooi zegt: Bewegen is goed voor lichaam en geest, doe je het met andere dan geniet je het meest!

Roberto, already for the writing of my master thesis, you played a critical role. We met during my Erasmus in Milan and the first time I only understood you were somebody who did something with computers. That somebody became the champion of my senior internship as you were able to carefully bring back to life my laptop (on which I spilled some tea). Thanks to you, I could continue to work on my thesis, but also, thanks to you, it became more difficult for me to focus. After my graduation I moved back to Milan, to live with you. That decision was not easy, but I am so happy I followed my heart. You did not only show how to cook properly and enjoy la dolce vita, but you also showed me the meaning of true amore. Now we are seven years later, and you still make me very happy Mr. Campione! I am very thankful you took the courage to leave your beautiful country and move with me to the Netherlands for this PhD adventure. Like seven years ago, your endless support and sweet encouragements helped me grow and reach this thesis finish line. Grazie mille per tutto quello che sei e tutto quello che fai per me! Hai un cuore molto grande e grazie a te, mia vita è pieno di risate, piacere, vino buono e bellezza. Anche grazie per tutte le tazze di tè che mi hai fatto! Sono molto fortunata di essere tua moglie e sono sicuro che ancora tante belle cose ci stanno aspettando. Ik hou van jou!! 


\section{ABOUT THE AUTHOR}

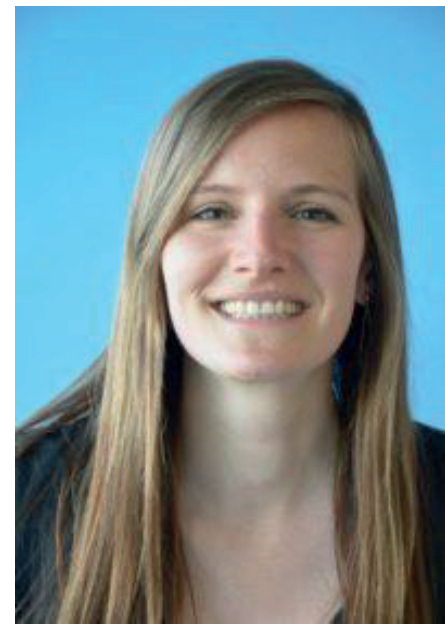

Lotte Grevendonk was born on May 27th, 1991, in Lommel, Belgium. In 2014 she graduated with a Master's degree in Biomedical Sciences at Hasselt University. During her junior internship, she was involved in clinical and molecular research on the effect of exercise therapy on patients with multiple sclerosis. For her Master's thesis project, she was selected for an international training program in the Epidemiology, Epigenetics and Toxicology Lab of Prof. Bollati at the University of Milan. There she performed her research project in the framework of the Environage birth cohort of Prof. Nawrot, investigating mitochondrial oxidative DNA damage caused by particulate air pollution exposure during pregnancy. Afterwards, she worked at the Laboratory of Genetics of Complex Neurological Disorders of Doctor Martinelli Boneschi at the San Raffaele Scientific Institute in Milan. In this research group, she was involved in the identification of relevant genetic variants in multiple sclerosis.

In September 2016, she started her PhD research at the Department of Nutrition and Movement Sciences at Maastricht University (NUTRIM, School of Nutrition and Translational Research in Metabolism), under the supervision of Dr. Joris Hoeks and Prof. Dr. Patrick Schrauwen. During her PhD, within the Diabetes and Metabolism Research Group, Lotte focused on the relationship between skeletal muscle mitochondrial metabolism and muscle health in older adults. As described in this thesis, her research aimed to understand the aging processes in the muscles better and investigate how healthy aging can be promoted. Upon finalization of her thesis, since May 2021, Lotte is continuing her scientific career as a research coordinator at the Department of Anesthesiology at Maastricht University Medical Center. 


\section{LIST OF PUBLICATIONS}

Grevendonk L, Connell NJ, McCrum C, Fealy CE. Fealy, Bilet L, Bruls YMH, Mevenkamp J, Schrauwen-Hinderling VB, Jörgensen JA, Moonen-Kornips E, Schaart G, Havekes B, de Vogel-van den Bosch J, Bragt MCE, Meijer K, Schrauwen, Joris Hoeks J. Impact of aging and exercise on skeletal muscle mitochondrial capacity, energy metabolism, and physical function. Nat Commun. 2021 Aug 6;12(1):4773. doi: 10.1038/s41467-021-24956-2. PMID: 34362885; PMCID: PMC8346468

Connell NJ, Grevendonk L, Fealy CE, Moonen-Kornips E, Bruls YMH, SchrauwenHinderling VB, de Vogel J, Hageman R, Geurts J, Zapata-Perez R, Houtkooper RH, Havekes B, Hoeks J, Schrauwen P. NAD+-Precursor Supplementation With LTryptophan, Nicotinic Acid, and Nicotinamide Does Not Affect Mitochondrial Function or Skeletal Muscle Function in Physically Compromised Older Adults. J Nutr. 2021 Jun 30:nxab193. doi: 10.1093/jn/nxab193. Epub ahead of print.

Gerards MHG, Meijer K, Karamanidis K, Grevendonk L, Hoeks J, Lenssen AF, McCrum C. Adaptability to Balance Perturbations During Walking as a Potential Marker of Falls History in Older Adults. Front Sports Act Living. 2021 May 19;3:682861. doi: 10.3389/fspor.2021.682861.

Lagerwaard B, van der Hoek MD, Hoeks J, Grevendonk L, Nieuwenhuizen AG, Keijer J, de Boer VCJ. Propionate hampers differentiation and modifies histone propionylation and acetylation in skeletal muscle cells. Mech Ageing Dev. 2021 Jun;196:111495. doi: 10.1016/j.mad.2021.111495. Epub 2021 Apr 28.

McCrum C, Karamanidis K, Grevendonk L, Zijlstra W, Meijer K. Older adults demonstrate interlimb transfer of reactive gait adaptations to repeated unpredictable gait perturbations. Geroscience. 2020 Feb;42(1):39-49. doi: 10.1007/s11357-01900130-x. Epub 2019 Nov 27. 
Motta V, Bonzini M, Grevendonk L, lodice S, Bollati V. Epigenetics applied to epidemiology: investigating environmental factors and lifestyle influence on human health. Med Lav. 2017 Feb 15;108(1):10-23. doi: 10.23749/mdl.v108i1.6072.

Grevendonk L, Janssen BG, Vanpoucke C, Lefebvre W, Hoxha M, Bollati V, Nawrot TS. Mitochondrial oxidative DNA damage and exposure to particulate air pollution in mother-newborn pairs. Environ Health. 2016 Jan 20;15:10. doi: 10.1186/s12940016-0095-2.

Wens I, Dalgas U, Vandenabeele F, Grevendonk L, Verboven K, Hansen D, Eijnde BO. High Intensity Exercise in Multiple Sclerosis: Effects on Muscle Contractile Characteristics and Exercise Capacity, a Randomised Controlled Trial. PLoS One. 2015 Sep 29;10(9):e0133697. doi: 10.1371/journal.pone.0133697.

Wens I, Dalgas U, Vandenabeele F, Krekels M, Grevendonk L, Eijnde BO. Multiple sclerosis affects skeletal muscle characteristics. PLoS One. 2014 Sep 29;9(9):e108158. doi: 10.1371/journal.pone.0108158.

Fealy CE, Grevendonk L, Hoeks J, Hesselink MKC. Skeletal muscle mitochondrial network dynamics in metabolic disorders and aging. Trends Mol Med. 2021 Aug 17:S1471-4914(21)00198-2. doi: 10.1016/j.molmed.2021.07.013. Epub ahead of print. PMID: 34417125. 


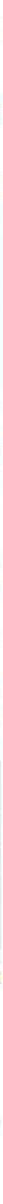

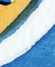

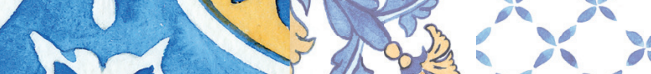
10 (n) 2 ( $)$ (1) (द) 5 कर 120
rov 150
- 205 10,2 $\beta 3$ (a) (3) 2028

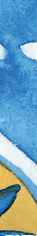

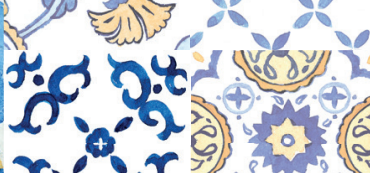

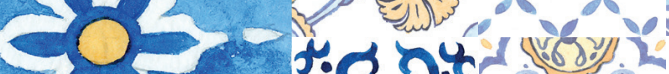

)

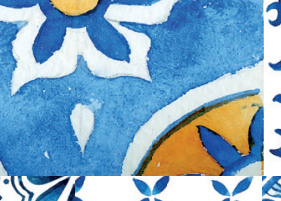
शी $\mathrm{Sac}$

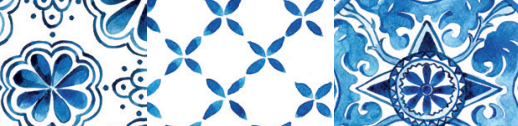

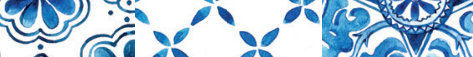

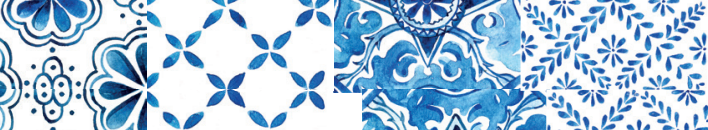

और $x$ और

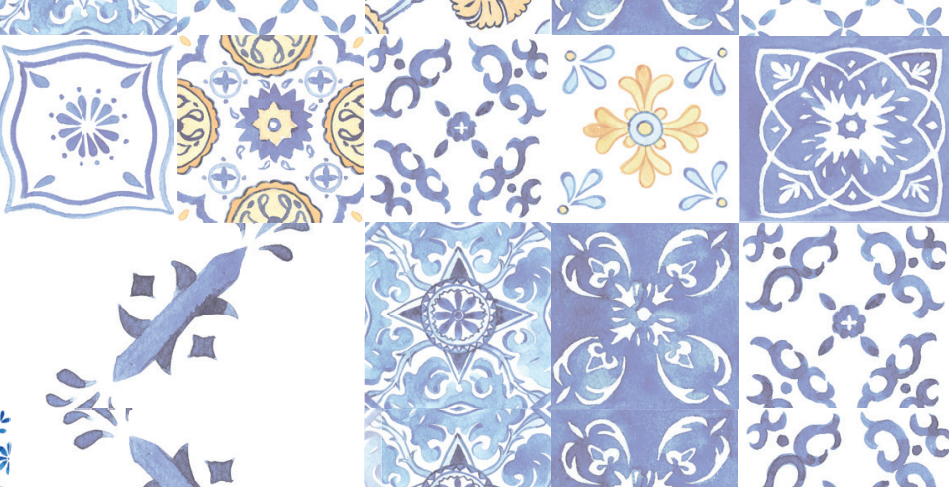

